\title{
Potentiating Therapeutic Effects of Epidermal Growth Factor Receptor Inhibition in Triple-Negative Breast Cancer
}

\author{
Kyu Sic You ${ }^{1,2,+} \mathbb{D}$, Yong Weon $\mathrm{Yi}^{3,+}{ }^{(\mathbb{D}}$, Jeonghee Cho ${ }^{3} \mathbb{C}$, Jeong-Soo Park ${ }^{1, *}$ and Yeon-Sun Seong ${ }^{1,2,3, * \mathbb{C}}$ \\ 1 Department of Biochemistry, College of Medicine, Dankook University, \\ Cheonan 31116, Chungcheongnam-do, Korea; kisuhezu@gmail.com \\ 2 Graduate School of Convergence Medical Science, Dankook University, \\ Cheonan 3116, Chungcheongnam-do, Korea \\ 3 Department of Nanobiomedical Science, Dankook University, Cheonan 31116, Chungcheongnam-do, Korea; \\ dragon101@gmail.com (Y.W.Y.); jeonghee.cho@dankook.ac.kr (J.C.) \\ * Correspondence: jeongsp@dankook.ac.kr (J.-S.P.); seongys@dankook.ac.kr (Y.-S.S.); \\ Tel.: +82-41-550-3876 (J.-S.P.); +82-41-550-3875 (Y.-S.S.) \\ + These authors contributed equally to this work.
}

Citation: You, K.S.; Yi, Y.W.; Cho, J.; Park, J.-S.; Seong, Y.-S. Potentiating Therapeutic Effects of Epidermal Growth Factor Receptor Inhibition in Triple-Negative Breast Cancer. Pharmaceuticals 2021, 14, 589. https:// doi.org/10.3390/ph14060589

Academic Editor: Massimiliano Tognolini

Received: 6 May 2021

Accepted: 14 June 2021

Published: 18 June 2021

Publisher's Note: MDPI stays neutral with regard to jurisdictional claims in published maps and institutional affiliations.

Copyright: () 2021 by the authors. Licensee MDPI, Basel, Switzerland. This article is an open access article distributed under the terms and conditions of the Creative Commons Attribution (CC BY) license (https:// creativecommons.org/licenses/by/ $4.0 /)$.

\begin{abstract}
Triple-negative breast cancer (TNBC) is a subset of breast cancer with aggressive characteristics and few therapeutic options. The lack of an appropriate therapeutic target is a challenging issue in treating TNBC. Although a high level expression of epidermal growth factor receptor (EGFR) has been associated with a poor prognosis among patients with TNBC, targeted anti-EGFR therapies have demonstrated limited efficacy for TNBC treatment in both clinical and preclinical settings. However, with the advantage of a number of clinically approved EGFR inhibitors (EGFRis), combination strategies have been explored as a promising approach to overcome the intrinsic resistance of TNBC to EGFRis. In this review, we analyzed the literature on the combination of EGFRis with other molecularly targeted therapeutics or conventional chemotherapeutics to understand the current knowledge and to provide potential therapeutic options for TNBC treatment.
\end{abstract}

Keywords: anticancer; combination therapy; epidermal growth factor receptor (EGFR); anti-EGFR therapy; EGFR inhibitor (EGFRi); EGFRi resistance; triple-negative breast cancer (TNBC)

\section{Introduction}

The epidermal growth factor receptor (EGFR) family (also known as the human epidermal growth factor receptor (HER) or erythroblastic leukemia viral oncogene homolog (ERBB) family) belongs to the receptor tyrosine kinase (RTK) superfamily, which consists of 58 transmembrane RTK proteins [1]. The EGFR/ERBB family members include EGFR (HER1/ERBB1), HER2 (ERBB2), HER3 (ERBB3), and HER4 (ERBB4) [2-5]. Under normal physiological conditions, the EGFR family members are activated by homo- and heterodimerization among them induced by their cognate ligands, such as epidermal growth factor (EGF), transforming growth factor alpha (TGF- $\alpha$ ), or amphiregulin (AREG), binding to their ectodomain (extracellular domain) [2,6]. After ligand binding, the activated EGFR dimer triggers multiple downstream signaling pathways, including the phosphatidylinositol-3-kinase (PI3K)/v-akt oncogene homolog (AKT)/mammalian target of rapamycin complex 1 (mTOR1) pathway, the rat sarcoma (RAS)/v-raf-1 murine leukemia viral oncogene homolog (RAF)/MAPK/ERK kinase (MEK)/extracellular signal-regulated kinase (ERK) pathway, and the Janus kinase (JAK)/signal transducer and activator of transcription 3 (STAT3) pathway, which are known to play key roles in various cellular responses such as cell proliferation, cell cycle progression, cell survival, and cell motility [2,6-9]. Aberrant activation of EGFR caused by various alterations such as copy number amplification, mutations, and protein overexpression has been reported in the majority of human cancers, including bladder, breast, colon, head and neck, kidney, liver, lung, 
ovary, pancreas, prostate, and stomach cancers [10]. In addition, inhibition of the oncogenic activity of EGFR with targeted drugs has been proven to be an effective clinical strategy in treating tumors driven by abnormal EGFR activation. However, the efficacy of this clinical tactic is quite limited due to the rapid emergence of drug resistance in patients following drug treatment. In lung adenocarcinoma, several resistance mechanisms of EGFR-targeted drugs such as erlotinib and osimertinib (EGFR-targeted small-molecule inhibitors) have been well established [11,12]. These include acquisition of additional mutations to EGFR such as T790M and C797S as resistance mechanisms to erlotinib and osimertinib, respectively $[13,14]$. Several concurrent genomic alterations including $M E T$ amplification or anexelekto (AXL) overexpression have also been identified as major drug resistance mechanisms [15].

Triple-negative breast cancer (TNBC) is clinically defined as a breast cancer subtype that lacks expression of the estrogen receptor (ER) and progesterone receptor (PR) and has no amplification of HER2 [16,17]. TNBC accounts for approximately $15-20 \%$ of diagnosed breast cancers [16-21]. However, few targeted therapies with limited clinical outcomes have been approved for TNBC treatment [22]. In addition, more than 50\% of cases of TNBC are known to express a high level of EGFR, which is associated with a poor prognosis $[4,5,16,21,23,24]$. EGFR expression has also been implicated with an unfavorable response to chemotherapy in patients with TNBC [25]. TNBC has been classified into at least six molecular subtypes, including basal-like 1 and 2 (BL1 and BL2), immunomodulatory (IM), luminal androgen receptor (LAR), mesenchymal (M), and mesenchymal stem-like (MSL) subtypes [19,26]. This classification was further refined into four subgroups, including BL1, BL2, M, and LAR, using histopathological quantification and laser-capture microdissection of clinical samples, since the IM and MSL subtypes have been identified to be contributed from infiltrating lymphocytes and tumor-associated stromal cells, respectively [27]. Continuous efforts to stratify molecular subtypes of heterogenous TNBC are still ongoing (reviewed in [28-30]).

Although activated EGFR signaling is observed in the BL2 and MSL subtypes of TNBC [19], TNBC has intrinsic resistance to anti-EGFR therapies [31], which has been supported by the disappointing outcomes of earlier attempts to treat TNBC with anti-EGFR monotherapies [32-35]. Thus, these results suggest that alternative oncogenic signaling initiated by receptors or downstream effectors may be the potential mechanism associated with the inefficacy of EGFR-targeted therapy against TNBC [36]. Consistent with this notion, various drug combination strategies to overcome resistance to EGFR-targeted drugs are currently under investigation.

In this report, we reviewed the recent progress of combination approaches related to anti-EGFR therapies for TNBC in 73 published studies. These publications were further analyzed to explore the current knowledge on the therapeutic windows of potentiating EGFR inhibition using drug combinations for TNBC treatment. Since multigeneration EGFR tyrosine kinase inhibitors (TKIs) and anti-EGFR antibody therapeutics have already been approved, the development of a combination strategy may provide an alternative therapeutic option to treat TNBC.

\section{Anti-EGFR Therapeutics}

To date, four anti-EGFR antibodies and twelve EGFR TKIs have been approved globally for treatment of various human cancers (Figure 1).

\subsection{Anti-EGFR Antibody Therapeutics}

Currently, three anti-EGFR antibodies have been approved by the US Food and Drug Administration (FDA), including cetuximab (Erbitux $\left.{ }^{\circledR}\right)$, panitumumab (Vectibix ${ }^{\circledR}$ ), and necitumumab (Portrazza ${ }^{\circledR}$ ) (Table 1) [37]. All of these antibody therapeutics are infused intravenously over the recommended time period [38,39]. Therapeutic anti-EGFR antibodies have been proposed to suppress the enzymatic activity of EGFR by the following mechanisms: (1) blockage of ligand binding to EGFR; (2) steric inhibition of homo- or 
heterodimerization among EGFR family members; (3) enhancement of EGFR internalization and subsequent degradation; (4) induction of the complement-dependent cytotoxicity (CDC) pathway; (5) induction of $G_{1}$ cell cycle arrest; (6) inhibition of proangiogenic factor expression; (7) induction of apoptosis; (8) induction of antibody-dependent cellular cytotoxicity (ADCC) by natural killer (NK) cells or monocytes or macrophages; or (9) induction of DNA damage [37].

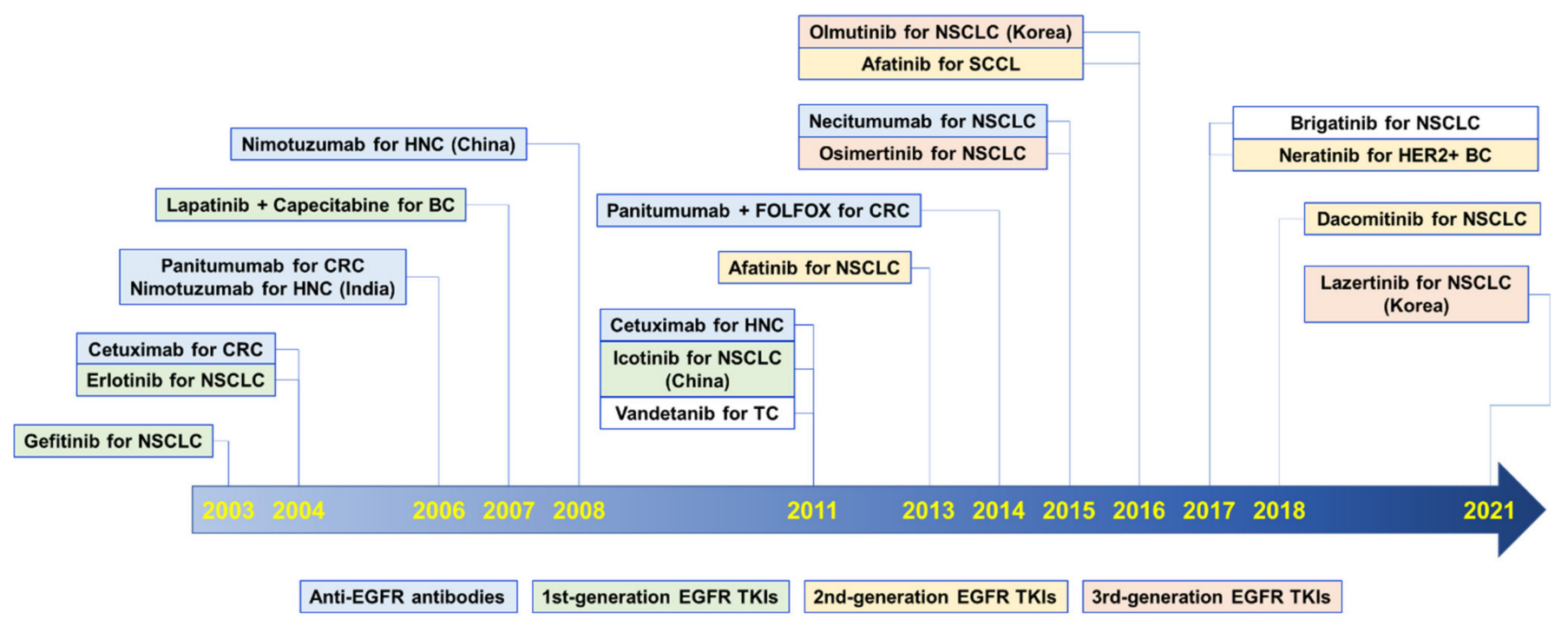

Figure 1. Milestones of anti-EGFR therapeutics approved globally. Some important milestones of regulatory approval for EGFR inhibitors are presented. See Tables 1 and 2 for more details. If not specified in parentheses, anti-EGFR therapeutics were approved by the US Food and Drug Administration (US FDA). Abbreviations: BC, breast cancer; CRC, colorectal cancer; HNC, head and neck cancer; NSCLC, non-small cell lung cancer; TC, thyroid cancer; TKIs, tyrosine kinase inhibitors.

Cetuximab is the first FDA-approved anti-EGFR antibody therapeutic. It is an IgG1 chimeric (mouse-/human) monoclonal antibody that potentially inhibits EGF from binding to EGFR and disrupts EGFR dimerization, leading to inhibition of its cognate downstream signaling activation [40,41]. It also induces ADCC by NK cells and neutrophils [42].

Panitumumab is the first fully human IgG2k monoclonal antibody specific to the extracellular ligand-binding domain of EGFR, blocking the downstream signaling pathways [43]. The threshold level of EGFR for antitumor effects of panitumumab was found to be more than 17,000 receptors per cell in xenograft models using human cancer cells [44]. Panitumumab is the only FDA-approved IgG2 anti-EGFR antibody, and unlike cetuximab, it does not induce ADCC [42].

Necitumumab is the most recently FDA-approved IgG1 monoclonal anti-EGFR antibody and is used in combination with gemcitabine and cisplatin for the first-line treatment of patients with advanced squamous NSCLC [39]. Necitumumab is an EGFR antagonist that binds to EGFR and inhibits the binding of EGFR to its ligands, preventing EGFR dimerization and activation. Necitumumab has also been demonstrated to induce ADCC in EGFR-positive cells in vitro [39].

Nimotuzumab is a humanized IgG1 monoclonal anti-EGFR antibody that has been approved for the following cancer types: (1) squamous cell carcinoma of the head and neck (SCCHN) in India, Cuba, Argentina, Colombia, Ivory Coast, Gabon, Ukraine, Peru, and Sri Lanka; (2) pediatric and adult glioma in Cuba, Argentina, Philippines, and Ukraine; (3) nasopharyngeal cancer in China [45]. Similar to other IgG1 anti-EGFR antibodies, it blocks ligand binding to EGFR, exerting an antitumor effect, and can also induce ADCC [42,45,46]. 
Table 1. Approved anti-EGFR antibody therapeutics.

\begin{tabular}{|c|c|c|c|c|c|}
\hline First Appr & Anti-EGFR Antibodies & $K_{D}{ }^{1}$ in $\mathrm{nM}$ & Developer & \multicolumn{2}{|c|}{ Indications Approved by the US FDA ${ }^{2}$} \\
\hline 2004 & $\begin{array}{c}\text { Cetuximab } \\
\left(\text { Erbitux }^{\circledR},{ }, \mathrm{ICM}-\mathrm{C} 225\right)\end{array}$ & $0.201[40] ; 1.8[46]$ & ImClone Systems & $\begin{array}{l}2004 \\
2011\end{array}$ & $\begin{array}{l}\text { Advanced } \mathrm{CRC}^{3} \\
\text { Late-stage } \mathrm{HNC}^{4}\end{array}$ \\
\hline 2006 & $\begin{array}{c}\text { Panitumumab } \\
\left(\text { Vectibix }^{\circledR}, \mathrm{ABX}-\mathrm{EGF}\right)\end{array}$ & $0.05[47]$ & Abgenix & $\begin{array}{l}2006 \\
2014 \\
2017\end{array}$ & $\begin{array}{l}\text { Metastatic CRC } \\
\text { First-line use of panitumumab } \\
\text { and FOLFOX for wild-type } \\
\text { KRAS metastatic CRC } \\
\text { Wild-type KRAS metastatic } \\
\text { CRC }\end{array}$ \\
\hline 2006 & $\begin{array}{l}\text { Nimotuzumab } \\
\text { (TheraCIM, h-R3, } \\
\text { BIOMAb EGFR) }\end{array}$ & $21[46]$ & $\begin{array}{c}\text { CIMYM } \\
\text { BioSciences }\end{array}$ & $\begin{array}{l}2006 \\
2008\end{array}$ & $\begin{array}{l}\text { HNC by the Central Drugs } \\
\text { Standard Control Organization } \\
\text { (CDSCO), India } \\
\text { HNC by the China Food and } \\
\text { Drug Administration (CFDA) }\end{array}$ \\
\hline 2015 & $\begin{array}{c}\text { Necitumumab } \\
\left(\text { Portrazza }^{\circledR}\right)\end{array}$ & $6.1[48]$ & Eli Lilly & 2015 & Advanced squamous NSCLC \\
\hline
\end{tabular}

${ }^{1} K_{D}$, dissociation constant; ${ }^{2}$ https: / / www.drugs.com/, accessed on 15 April 2021; ${ }^{3}$ CRC, colorectal cancer; ${ }^{4} \mathrm{HNC}$, head and neck cancer;

5 https://www.pharmacodia.com/, accessed on 15 April 2021.

\subsection{Small-Molecule TKIs}

As of December 23, 2020, 62 small-molecule protein kinase inhibitors (PKIs) have been approved by the US FDA [49]. Among them, small-molecule TKIs for EGFR, including gefitinib, erlotinib, afatinib, osimertinib, and dacomitinib (Table 2), have been initially approved to treat non-small cell lung cancer (NSCLC) [50]. The EGFR/HER2 dual inhibitors, lapatinib and neratinib, which have similar half-maximal inhibitory concentration $\left(\mathrm{IC}_{50}\right)$ values for EGFR and HER2, have been approved for patients with HER2-positive breast cancer (Table 2). To date, first-to-fourth-generation EGFR TKIs have been developed [51]. Unlike anti-EGFR antibodies, all EGFR TKIs are orally bioavailable [52], making it easy for patients to take the medicine.

\subsubsection{First-Generation EGFR TKIs}

The first-generation EGFR TKIs are oral, reversible inhibitors that block the binding of adenosine triphosphate (ATP) to the tyrosine kinase domain [50]. These include gefitinib, erlotinib, lapatinib, and icotinib [50,51].

Gefitinib (Iressa ${ }^{\circledR}$ ) is the first US FDA-approved small-molecule TKI [50]. It received accelerated US FDA approval in 2003 as a monotherapy for patients with advanced NSCLC after failure of both platinum and docetaxel therapies [50]. However, gefitinib was voluntarily withdrawn in 2012 due to its limited efficacy in subsequent clinical trials $[50,53]$. More recently, gefitinib received US FDA approval for the first-line treatment of patients with metastatic NSCLC with EGFR exon 19 deletion (ex19del) or exon 21 substitution (L858R) mutations $[50,53]$. This new approval is because clinical trials have demonstrated the efficacy of gefitinib in patients carrying EGFR mutations [54-57].

Erlotinib (Tarceva ${ }^{\circledR}$ ) was discovered in 1997 as a selective EGFR inhibitor [58]. It was approved by the US FDA in 2004 to treat patients with advanced or metastatic NSCLC after the failure of at least one prior chemotherapy $[59,60]$. Due to its superiority compared with other chemotherapeutics in response and progression-free survival (PFS), erlotinib was further approved for the first-line therapy of patients with NSCLC with EGFR mutations [50].

Lapatinib $\left(\right.$ Tykerb $\left.^{\circledR}\right)$ is a potent dual EGFR/HER2 inhibitor [61]. Lapatinib was approved by the US FDA in 2007 for breast cancer in combination with capecitabine (Xeloda) [62]. Crystal structure analysis revealed that the conformation of lapatinib-bound EGFR is different from that of erlotinib-bound EGFR, leading to much slower dissociation of lapatinib from the complex than erlotinib [63]. Consistently, lapatinib downregulates tyrosine phosphorylation for much longer periods than erlotinib and gefitinib. 
Icotinib $\left(\right.$ Conmana ${ }^{\circledR}$ ) is another first-generation EGFR TKI that is solely approved (in 2011) and marketed in China for the second- or third-line therapy of metastatic NSCLC [50]. Icotinib is structurally similar to gefitinib and erlotinib but is less toxic and better tolerated than gefitinib [64]. In 2014, it was further approved for the first-line treatment of patients with EGFR-mutant NSCLC in China [65].

\subsubsection{Second-Generation EGFR TKIs}

Second-generation EGFR TKIs are irreversible kinase inhibitors that covalently bind to the kinase domain of EGFR [66]. Afatinib (Tovok ${ }^{\circledR}$, BIBW2992) is an ATP-competitive irreversible TKI that covalently binds and irreversibly blocks enzymatically active EGFR family members (EGFR, HER2, and HER4) [67]. It was approved by the US FDA in 2013 for late stage NSCLC, in 2016 for squamous cell carcinoma of the lung, and in 2018 for EGFR mutation-positive NSCLC (Table 2). The superiority of afatinib in terms of PFS and responses over pemetrexed plus cisplatin [68] or gemcitabine plus cisplatin [69] for patients with EGFR-mutant NSCLC was demonstrated in two clinical trials.

Neratinib $\left(\right.$ Nerlynx $\left.{ }^{\circledR}\right)$ is an irreversible HER2 and EGFR TKI approved for the treatment of HER2-positive breast cancer [70]. In the EU and the US, neratinib was approved as an adjuvant treatment for adults with early-stage, hormone-receptor-positive, HER2overexpressed or amplified breast cancer and adults with less than one year from prior adjuvant trastuzumab-based therapy [71]. In the US, neratinib in combination with capecitabine was also approved for adults with advanced or metastatic HER2-positive breast cancer [72].

Dacomitinib (Vizimpro ${ }^{\circledR}$ ) is an irreversible pan-HER inhibitor (Table 2). It covalently binds to a cysteine residue in the ATP pocket of the kinase domain. Similar to afatinib, it blocks signal transduction from both homo- and heterodimers of all members of the EGFR/HER family [73,74]. Dacomitinib demonstrates longer pharmacodynamic effects than the first-generation TKIs [75].

\subsubsection{Third-Generation EGFR TKIs}

To overcome the limited efficacy of second-generation EGFR TKIs for EGFR-activating mutations such as T790M, L858R, or ex19del, third-generation mutation-selective EGFR TKIs including alflutinib (AST2818), almonertinib (HS-10296), avitinib (AC0010), lazertinib (Leclaza ${ }^{\circledR}$; YH25448), olmutinib (Olita ${ }^{\mathrm{TM}}$ ), osimeritinib (Tagrisso ${ }^{\circledR}$, AZD9291), narzatinib (EGF816), rociletinib (CO-1686), and WZ4002 have been developed [51,76].

Osimertinib is the first FDA-approved third-generation EGFR TKI for EGFR ${ }^{\mathrm{T790M}}$ mutation-positive NSCLC and was approved in 2015 [76]. The T790M mutation may confer intrinsic (de novo or primary) or acquired (or secondary) resistance following firstline treatment with other EGFR TKIs, such as gefitinib and afatinib [13]. Although it is effective in treating locally advanced or metastatic NSCLC with T790M or other activating EGFR mutations [77], resistance to osimertinib usually occurs due to a C797S mutation approximately 10 months after treatment [14].

Olmutinib was approved in Korea in May 2016 for patients previously treated with an EGFR TKI with locally advanced or metastatic NSCLC with an EGFR ${ }^{\mathrm{T} 790 \mathrm{M}}$ mutation [78]. Olmutinib has been granted accelerated approval by the Korea Ministry of Food and Drug Safety (MFDS) under the condition that a phase 3 clinical trial would be conducted postmarketing. The US FDA also granted olmutinib a breakthrough therapy designation for NSCLC in December 2015 [78]. The C797S mutation of EGFR also develops after treatment with olmutinib [14].

Lazertinib was also conditionally approved in Korea in January 2021 for patients with NSCLC with EGFR ${ }^{\mathrm{T} 790 \mathrm{M}}$ mutations (Table 2). Lazertinib has been reported to have a higher selectivity and fewer off-target effects than osimertinib [79]. The phase 1-2 study of 127 patients in 14 hospitals in Korea reported a tolerable safety profile with a median PFS of 9.5 months for patients with $E G F R^{T 790 \mathrm{M}}$-positive NSCLC versus 5.4 months for those with $E G F R^{\mathrm{T} 790 \mathrm{M}}$-negative NSCLC [80]. Resistance to lazertinib caused by the C797S mutation of EGFR has also been reported [81]. 


\subsubsection{Fourth-Generation EGFR TKIs}

Allosteric inhibitors are under development as fourth-generation EGFR TKIs to overcome $E G F R^{\mathrm{L} 858 \mathrm{R}}, E G F R^{\mathrm{T} 790 \mathrm{M}}$, and $E G F R^{\mathrm{C} 797 \mathrm{~S}}$-mediated resistance [51]. BBT-176 is a firstin-class fourth-generation EGFR TKI designed to inhibit EGFR ${ }^{C 797 S}$ mutation that arises following osimertinib treatment in patients with NSCLC and is in a phase 1-2 clinical trial for advanced NSCLC [82]. The clinical trial was designed to use BBT-176 alone or in combination with cetuximab (NCT04820023). In preclinical studies, it showed strong antitumor activity in xenografts with EGFR ${ }^{\text {ex19del/T790M/C797S }}$ and EGFR ${ }^{\text {L858R/T790M/C797S }}$, and its efficacy was enhanced in combination with anti-EGFR antibody. BBT-176 was discovered by the Korea Research Institute of Chemical Technology (KRICT) and has been licensed by Bridge Biotherapeutics, with worldwide exclusive rights for further development.

EAI001 and EAI045 are non-ATP competitive inhibitors that bind allosterically to EGFR with higher specificity for mutant EGFR ${ }^{\mathrm{T} 790 \mathrm{M} / \mathrm{C} 797 \mathrm{~S}}$ than for wild-type EGFR [83,84]. Another mutant-selective EGFR allosteric inhibitor, JBJ-04-125-02, blocks the EGFR ${ }^{\text {L858R/T790M/C797S }}$ signaling both in vitro and in vivo and has antitumor activity against an NSCLC xenograft tumor [85]. Further clinical benefits may be addressed in the future.

\subsubsection{Multitargeting TKIs}

Examples of multitargeting TKIs with inhibitory activity against EGFR include brigatinib and vandetanib. Brigatinib (Alunbrig ${ }^{\circledR}$; AP26113) is a multi-kinase inhibitor that was originally developed as a TKI against anaplastic lymphoma kinase (ALK) [86]. Brigatinib further inhibits multiple tyrosine kinases including proto-oncogene tyrosine protein kinase ROS, fms-related receptor tyrosine kinase 3 (FLT3), and insulin-like growth factor 1 receptor (IGF1R), with $\mathrm{IC}_{50}$ values of $1.9 \mathrm{nM}, 2.1 \mathrm{nM}$, and $24.9 \mathrm{nM}$, respectively [86]. A drug screening against cells harboring EGFR C797S/T790M/ex19del identified that brigatinib also has inhibitory activity against mutant EGFR including C797S/ex19del $\left(\mathrm{IC}_{50}=39.9 \mathrm{nM}\right)$, ex19del $\left(\mathrm{IC}_{50}=43.7\right), \mathrm{C} 797 \mathrm{~S} / \mathrm{T} 790 \mathrm{M} / \mathrm{ex} 19 \mathrm{del}\left(\mathrm{IC}_{50}=67.2\right)$, and T790M/ex19del $\left(\mathrm{IC}_{50}=150.3\right)$ [87]. Brigatinib received accelerated approval by the US FDA in 2017 for ALK-positive (ALK+) metastatic NSCLC and was further approved in 2020 as a first-line treatment option for patients with ALK+ metastatic NSCLC (Table 2).

Vandetanib (Caprelsa ${ }^{\circledR}$; Zactima ${ }^{\circledR}$; ZD6474) is an orally available RTK inhibitor targeting vascular endothelial growth factor receptor 2/3 (VEGFR2/3), rearranged during transfection (RET), and EGFR, which are important targets in thyroid cancer (Table 2) [88,89]. It was the first FDA-approved medication for the treatment of advanced medullary thyroid cancer (MTC) [90]. Importantly, its in vivo antitumor effects are indirectly mediated by reducing the production of EGFR-induced angiogenic growth factor [89]. Vandetanib can prolong the PFS of patients with aggressive MTC and can stabilize the disease [90]. Vandetanib has been found to be effective in inducing in vivo tumor regression in TNBC PDX models with high expression of RET or EGFR with concordant suppression of RET/EGFR phosphorylation and MEK/ERK pathway activation [91]. Overexpression of RET was found in $<10 \%$ and $20-40 \%$ of TNBC- and HER2-positive breast cancer samples, respectively. 
Table 2. Approved anti-EGFR TKIs.

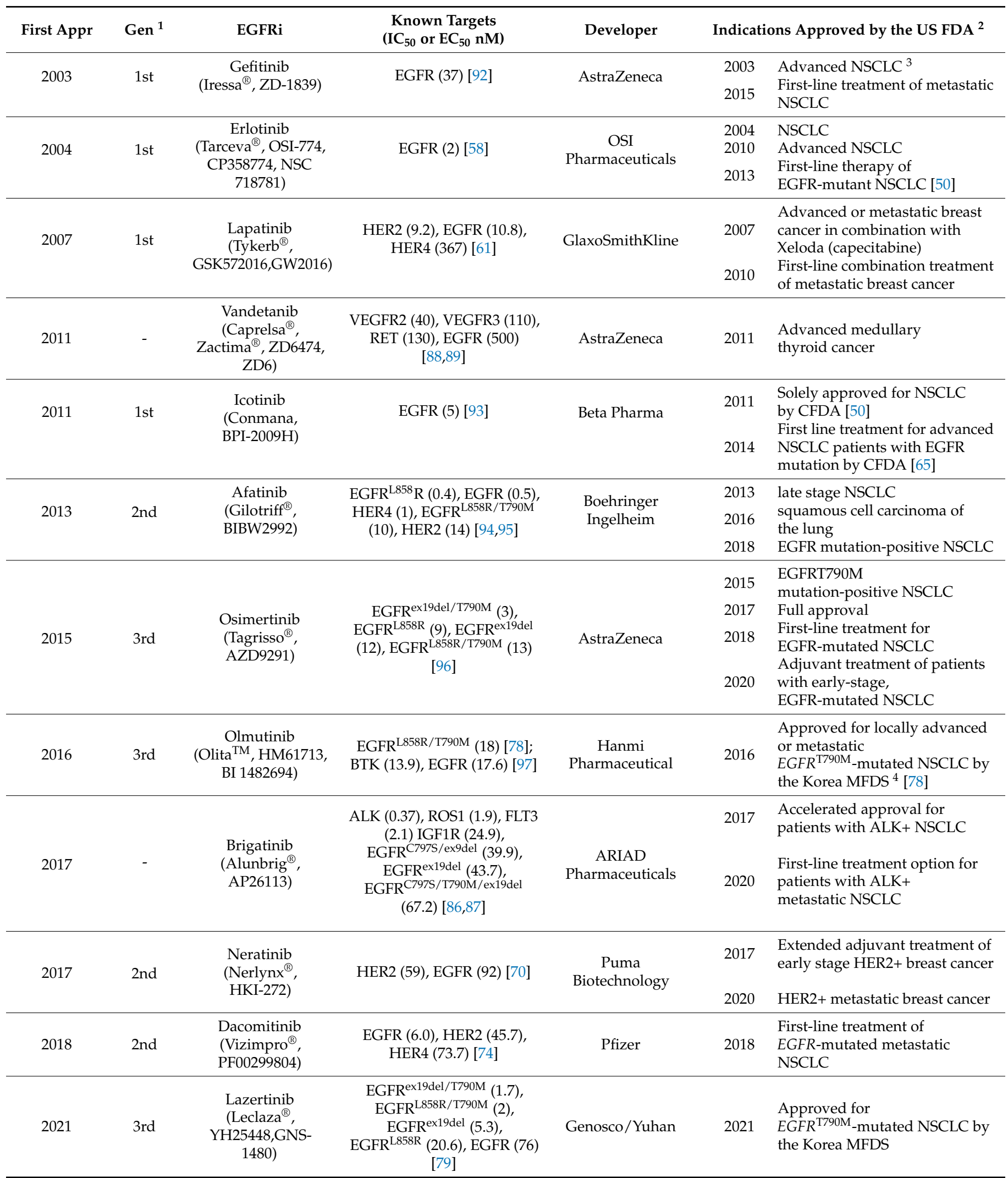

${ }^{1}$ Gen, generation; ${ }^{2}$ https: / /www.drugs.com/, accessed on 15 April 2021; ${ }^{3}$ NSCLC, non-small cell lung cancer; ${ }^{4}$ Ministry of Food and Drug Safety. 


\section{Resistance to Anti-EGFR Therapeutics}

Resistance of cancer to anticancer therapeutics is classified into two main categories: intrinsic (de novo or primary) resistance and acquired (secondary) resistance [98]. Current knowledge and management strategies have recently been described in detail [98]. Here, we provide a brief overview of cancer resistance to anti-EGFR therapies, especially in TNBC.

TNBC has been demonstrated to have an intrinsic resistance to anti-EGFR therapies, including both anti-EGFR antibodies and EGFR TKIs [31]. For example, a study with 20 TNBC cell lines reported that twelve $(60 \%)$ were classified as refractory to most of the 24 EGFR TKIs tested [99]. In addition, acquired resistance to anti-EGFR monotherapy has been reported in various types of cancers. For example, acquired resistance of NSCLC to EGFR inhibitors (EGFRis) develops within 9 to 14 months of treatment [85]. From the studies on NSCLC in which mechanisms of resistance to EGFRis have been extensively studied, intrinsic EGFRi resistance of TNBC may be due to (1) EGFR-activating mutations or amplification, (2) activation of bypass signaling pathways (e.g., MET amplification), or (3) activation of downstream pathways [50,76,77,100,101]. Rewiring of signal transduction pathways by dynamic signaling networks, including feedback loops and crosstalk, also contributes to unexpected adverse effects or EGFRi resistance in cancer cells $[102,103]$.

\subsection{EGFR Mutations or Amplification}

Mutations conferring EGFR TKI resistance were discovered several months after EGFR TKI therapy $[50,76,85,100,101]$. T790M, the gateway mutation, was found in $50-65 \%$ of patients who had acquired resistance to EGFR TKIs [104-106]. This mutation blocks binding of first-generation TKIs to EGFR by increasing the binding affinity of EGFR to ATP [106]. In addition, a tertiary mutation, EGFR ${ }^{\mathrm{C} 797 \mathrm{~S}}$, was detected in $20-25 \%$ of patients with NSCLC who received osimertinib $[107,108]$. C797 is the covalent binding site for all known second-generation EGFRis, while the C797S point mutation blocks the covalent binding of irreversible EGFR TKIs [107]. EGFR ${ }^{C 797 \mathrm{~S}}$-mediated resistance has also been reported in patients treated with olmutinib [109], rociletinib [110], and nazartinib [111].

In contrast to NSCLC, the incidence rate of EGFR-activating mutations in TNBC has been reported to be relatively low, at between $0 \%$ and $11.4 \%$ [112]. For example, a mutational analysis identified EGFR mutations in only 8 of 70 TNBC samples, with four samples demonstrating ex19 deletions, including 15-nucleotide deletions (del E746-A750) in 2 samples and 24-nucleotide deletions (del S752-I759) within the kinase domain in other samples. Furthermore, one sample showed inversion of the complementary strand of exon 19 and three samples showed exon 21 mutations, including one case of L858R and two cases of T847I [113]. Although positive staining for EGFR does not correlate well with EGFR mutations in these TNBC samples, the ex19del and exon 21 substitutions are commonly found in NSCLC and are good predictors of EGFR TKI sensitivity [114]. Additionally, no activating EGFR mutations were identified by tissue microarray and pyrosequencing analysis in $493 \mathrm{TNBC}$ cases [112]. In fact, EGFR mutations are very rare in patients with TNBC in various populations. including American [115], Australian [116], British [117], French [118,119], German [120], Korean [112,121,122], Chinese [113,123], Switzerland [124], and Japanese $[117,125,126]$ populations. Currently, intrinsic resistance of TNBC to antiEGFR monotherapies has been understood to be a consequence of activation of a bypass or downstream signaling pathway(s) rather than due to EGFR mutations.

Accumulation of nuclear EGFR has been linked to drug resistance in TNBC [127-130]. Nuclear EGFR activates the transcription of genes involved in cell cycle progression, such as cyclin D1 [131] and aurora kinase A (AURKA) [132]. Nuclear EGFR, independent of canonical signaling pathway, has been reported to increase DNA repair in response to cisplatin and ionizing radiation by increasing nuclear accumulation and activation of the DNA-dependent protein kinase catalytic subunit (DNA-PKcs) [133]. DNA-PKcs plays a critical role in non-homologous end-joining DNA repair [134]. Interestingly, cetuximab and gefitinib have been reported to inhibit nuclear accumulation of EGFR, leading to cytoplasmic retention and inhibition of DNA-PKcs $[135,136]$. In addition, EGFR has also been 
associated with two essential components of the homology-directed recombinational repair (HDR), the RAD51 (S. cerevisiae) homolog (RAD51), and the breast cancer susceptibility gene 1 (BRCA1). The EGFRi gefitinib decreased the protein stability of RAD51 and its mRNA levels [137]. Erlotinib has also been demonstrated to decrease HDR by reducing RAD51 foci and increasing cytoplasmic BRCA1 [138].

\subsection{Activation of Bypass Signaling Pathways}

Although anti-RTK antibodies block the binding of the cognate ligands to the ectodomain of RTKs, leading to shutdown of downstream signaling pathways, agonist-like or partial agonist effects of RTKs have also been reported [139-142]. This partial agonistic action may induce autophosphorylation of RTKs that activate downstream signaling pathways, eventually causing resistance to anti-RTK antibodies. For example, trastuzumab and cetuximab exert agonistic effects on HER2 and EGFR, respectively [143-145]. In addition, the agonistic action of an anti-RTK antibody may transactivate other RKTs, such as IGF1R or VEGFRs [142].

Amplification or activation of other RTKs, such as mesenchymal epithelial transition factor (MET, also known as hepatocyte growth factor receptor (HGFR)), HER2, IGF1R, fibroblast growth factor receptor (FGFR), and AXL, might be a potential mechanism of intrinsic resistance to anti-EGFR monotherapies in TNBC [15]. Amplification of these RTKs drives activation of their downstream targets, such as the PI3K/AKT/mTORC1, RAS/RAF/MEK/ERK, and JAK/STAT3 pathways [98,146-149].

MET overexpression has been found in various cancers in association with a poor prognosis and confers resistance not only to therapies targeting EGFR, BRAF, and MEK, but also to chemotherapies [148]. High expression of MET in clinical TNBC samples has been associated with poor overall survival (OS) and disease-free survival (DFS) [150,151]. A previous study demonstrated that HGF, the ligand for MET, is secreted from fibroblasts and activates MET in TNBC cells, leading to EGFRi resistance through EGFR-MET crosstalk [152]. The fact that knockdown of EGFR in TNBC cells abolished the HGF-dependent TNBC cell survival further supports the importance of EGFR-MET crosstalk in TNBC cells [152]. Activation of MET by HGF has also been reported to promote resistance to EGFRis in TNBC cells [153].

HER2 has also been identified to induce drug resistance via activation of nuclear factor E2-related factor-2 (NRF2) by a direct physical interaction, leading to induction of NRF2 target gene products, such as heme oxygenase 1 (HO1), cytochrome P450 3A4 (CYP3A4), glutathione S-transferase A2 (GSTA2), glutathione S-transferase P1 (GSTP1), multidrug resistance protein 1 (MDR1), multidrug resistance-associated protein 1 (MRP1), MRP4, and MRP5 [154]. NRF2 is a master transcription factor that regulates the expression of genes in oxidative stress responses, detoxification, and drug resistance [155].

Although HER3 has a catalytically inactive kinase domain, it serves as a signaling platform by heterodimerizing other receptors [156]. Overexpression of HER3 or its ligand heregulin (HRG, also known as neuregulin-1 (NRG1)), has been linked to EGFR TKI resistance [157-159]. Additionally, an increase in HER3 was identified in patients with TNBC treated with cetuximab or panitumumab [159]. A compensatory increase in and activation of HER3 has been identified as a result of gefitinib treatment in breast cancer cells through release of the AKT-mediated negative feedback loop [157]. Since AKT negatively regulates Forkhead box O (FOXO) transcription factors that are responsible for transcription of RTKs, such as HER3, IGF1R, FGFR2, and insulin receptor, blocking the PI3K/AKT pathway results in the expression of these RTKs in TNBC cells [160]. Activated proteintyrosine kinase 2 (PYK2 or focal adhesion kinase 2 (FAK2)) has also been reported to inhibit the binding of HER3 to neural precursor cell expressed developmentally downregulated protein 4 (NEDD4) [161]. Since NEDD4 is an E2 ubiquitin ligase for HER3, disruption of the HER3-NEDD4 interaction leads to blocking of the proteasome-dependent degradation of HER3. In addition, EGFR inhibition induced upregulation of HER3, leading to EGFRi 
resistance, and increased HER3 was associated with a decreased response of patients with TNBC to panitumumab or cetuximab in clinical studies [159].

Activated AXL has also been linked to intrinsic resistance to osimertinib [162,163]. Overexpression of AXL has been associated with acquired resistance to EGFRis in NSCLC [164,165]. In the TNBC cell line MDA-MB-231, AXL was found to be overexpressed and physically associated with EGFR [166]. Additionally, AXL knockdown (KD) reduced the EGFR-dependent activation of downstream signaling and the EGF-stimulated migration of MDA-MB-231 cells.

Adaptive kinome reprogramming has been established as a mechanism of kinase inhibitor resistance via upregulation of expression of different RTKs [167]. For example, blocking the RAS/RAF/MEK/ERK pathway may lead to destabilization of the oncogene product $\mathrm{v}$-myc avian myelocytomatosis viral oncogene homolog (MYC) through a proteasome-dependent manner in TNBC cells (see Section 3.3.1. Activation of the RAS/RAF/MEK/ERK pathway.) [168,169]. Since MYC-KD induces the expression of the platelet-derived growth factor receptor beta $(P D G F R \beta), V E G F R 2$, and platelet-derived growth factor subunit B (PDGFB) and increased tyrosine phosphorylation of PDGFR $\beta$, VEGFR2, HER3, and AXL in the TNBC cell line SUM159PT [168], the degradation of MYC by kinase inhibition causes resistance of cancer cells to the inhibition.

\subsection{Activation of Downstream Pathways}

The components in the downstream pathways of RTKs, such as the RAS/RAF/MEK/ERK, $\mathrm{PI} 3 \mathrm{~K} / \mathrm{AKT} / \mathrm{mTORC1}$, and JAK/STAT3 pathways, have been studied as intervening nodes either for monotherapy or combination therapy for TNBC cells [170-173]. However, the EGFinduced activation of the RAS/RAF/MEK/ERK and PI3K/AKT/mTORC1 pathways persists even in the presence of lapatinib in EGFRi-resistant TNBC cell lines, suggesting that EGFRi resistance occurs through the bypassing activation of downstream survival pathways [99].

\subsubsection{Activation of the RAS/RAF/MEK/ERK Pathway}

Single nucleotide point mutations in codons 12 and 13 of KRAS lead to amino acid substitutions including G12D, G12V, G12C, G12S, G12A, and G13D [36]. All of these mutations impair KRAS GTPase activity, causing accumulation of the hyperactive GTPbound form of KRAS proteins [174]. This accumulation of active KRAS results in the EGFR-independent constitutive activation of the RAS/RAF/MEK/ERK pathway.

Interestingly, only a small proportion of breast cancers have mutations in $K R A S(5 \%)$, HRAS (1\%), and BRAF (2\%) [175]. Nevertheless, hyperactivation of the RAS/RAF/MEK/ERK pathway is associated with TNBC [176]. However, its clinical relevance remains to be determined. The $3^{\prime}$ UTR, which contains a polymorphism in a let-7 miRNA complementary site of a KRAS variant has been linked to TNBC clinical samples with altered gene expression [177]. This variant was further associated with a low level of let-7 expression in TNBC. Interestingly, a recent study demonstrated that TNBC has higher KRAS signaling than ER-positive breast cancer [178]. In addition, patients with TNBC with enriched KRAS signaling gene sets are associated with inflammation and a favorable tumor-immune microenvironement, as well as improved DFS and OS [178].

Aberrant activation of the RAS/RAF/MEK/ERK pathway may induce stabilization of MYC via ERK-dependent phosphorylation at S62 in TNBC cells [168]. Thus, p-MYC could escape proteasomal degradation [169]. Furthermore, the RAS-mediated activation of the PI3K pathway leads to blocking of the inhibitory phosphorylation of MYC at T58 by GSK3 $\beta$, which is negatively regulated by AKT. In addition, the phosphorylation and stability of the beta-transducin repeat-containing protein 1 ( $\beta$-TrCP1), which antagonizes the E3 ubiquitin ligase FBXW7 to stabilize MYC [179], is also regulated by the PI3K/mTORC2 pathway in TNBC cells [171]. Overexpression of MYC has been reported in TNBC and is associated with drug resistance [180-182]. $\beta$-TrCP1 is also a F-box/WD repeat-containing protein (FBXW) subfamily member and the substrate-recognition subunit of the SKP1-cullin 1F-box protein (SCF) ubiquitin-ligase complex [183-185]. $\beta$-TrCP1 plays roles as a both 
an oncogene and a tumor suppressor in a tissue-specific and cellular-context-dependent manner. $\beta$-TrCP1-KD has been reported to suppress the growth of the MSL TNBC cell lines HS578T and MDA-MB-231 [171]. Overexpression of $\beta$-TrCP is known to promote tumorigenesis and is associated with various cancers such as breast, colorectal, gastric, hepatoblastoma, melanoma, pancreatic, and prostate cancers $[184,185]$. $\beta$-TrCP binds to phosphorylated DEP-domain-containing mTOR-interacting protein (DEPTO), a negative regulator of $\mathrm{mTORC} 1 / 2$, leading to degradation of DEPTOR [186-188]. In contrast, $\beta-\operatorname{TrCP}$ KD results in DEPTOR accumulation, which causes reduced mTOR and S6K activities and autophagy induction $[187,188]$.

\subsubsection{Activation of the PI3K/AKT/mTORC1 Pathway}

The PI3K/AKT/mTORC1 pathway is a major effector of the EGFR. Three hotspot PIK3CA (PI3K catalytic subunit)-activating mutations include E453K, E545K, and H1047R [189]. Mutation analyses of TNBC have found frequent mutations in PIK3CA ( 10\% to $24 \%)$ and phosphatase and tensin homolog $(P T E N)(\sim 8 \%)$ [190,191]. In addition, high frequencies of PTEN loss or inactivation (35\%) and AKT3 amplification (28\%) were also found in TNBC [192]. PTEN is a lipid phosphatase that inhibits the activity of PI3K by removing a phosphate from phosphatidylinositol $(3,4,5)$ triphosphate to form phosphatidylinositol $(4,5)$ bisphosphate [193]. PTEN serves as a tumor suppressor, and loss of PTEN activates the PI3K/AKT/mTORC1 pathway [194]. In fact, the PI3K/AKT/mTOR pathway has been reported to be activated in approximately $60 \%$ of patients with TNBC [167].

PIK3CA mutations have also been associated with resistance of TNBC cell lines to chemotherapy [195]. In addition, activating PIK3CA mutations in basal-like breast cancer were found to induce paracrine activation of AREG/EGFR/ERK signaling [196]. High levels of both AREG mRNA and protein were correlated with the mutation status of PIK3CA in breast cancer cell lines.

Among three isoforms (AKT1, AKT2, and AKT3), AKT3 has been identified as overexpressed both at the DNA and mRNA levels in TNBC [197]. In addition, high levels of p-AKT (T308) and p-AKT (S473), markers for the PI3K/AKT activation, are associated with TNBC [192].

\subsubsection{Activation of the NF-kB Pathway}

Aberrant activation of the nuclear factor kappa light chain enhancer of activated B cells (NF- $\kappa$ B) pathway also confers intrinsic and acquired resistance [198]. Furthermore, NF- $k B$ is constitutively active in many type of cancers [199]. The activity of NF- $\kappa B$ is negatively regulated by inhibitor of NF- $\kappa \mathrm{B}$ (I $\kappa \mathrm{B}$ ) through a complex formation. The dissociation of I $\mathrm{B}$ is controlled by the IKB kinase (IKK) complex-induced phosphorylation and subsequent degradation of p-IкB [199-201]. EGFR-NF- $\kappa$ B crosstalk in cancer cells has previously been identified, showing that (1) EGFR directly or indirectly activates NF- $\mathrm{B}$ in human various cells, including ER-negative breast cancer cells [202-210], while (2) the IKK/NF- $\mathrm{B}$ pathway activates EGFR signaling [211-214]. In addition, activated NF- $\kappa B$ confers EGFRi resistance. Inhibition of NF- $\mathrm{BB}$ consistently sensitizes cancer cells to EGFRis [202,215-219]. For example, NF- $\kappa B$ activates the expression of the anti-apoptotic proteins BCL2-like protein 1 isoform 1 (BCL2L1, also known as BCL-xL) and BCL2-related protein A1 (BCL2A1) in a Mucin 1 carboxy terminal subunit (MUC1-C)-dependent manner [220,221]. Notably, a high expression level of MUC1-C has been reported in TNBC [222,223]. Although the IKK/NF- $\mathrm{KB}$ pathway has been recognized as a potential therapeutic target for TNBC treatment $[209,217,224]$, further studies are needed to determine the effects of IKK/NF$\mathrm{B}$ inhibition on EGFRi resistance in TNBC. Our group very recently identified an IKK inhibitor as a sensitizer of EGFRi in TNBC cells (You et al., manuscript in preparation).

\subsubsection{Activation of the c-Jun N-Terminal Kinase (JNK) Pathway}

The activation of JNKs has been correlated with EGFR expression in TNBC [225] and leads to increased c-Jun mRNA associated with a decreased DFS among patients with 
TNBC [226]. The JNK pathway has been linked to increases in invasiveness, angiogenesis, and metastasis; cancer stem cell (CSC) phenotype; and acquired resistance to EGFR/HER2targeted therapies [226-230]. Of interest, the EGFR and HER2 dual inhibitor lapatinib has been reported to induce increased p-c-Jun and p-JNK in the TNBC cell line MDA-MB-231, resulting in resistance of these cells to lapatinib [231] (see Section 4.1.6. Combination with JAK Inhibitors).

\subsubsection{Activation of the Notch Pathway}

The Notch signaling pathway is composed of single-pass transmembrane Notch receptors in the signal receiving cells and their ligands jagged and delta-like proteins in the signal sensing cells $[232,233]$. Dysregulation or activation of the Notch signaling pathway is a hallmark for TNBC, with a strong correlation with aggressive clinicopathological features [232]. Four Notch receptors have been associated with tumor growth (Notch1, Notch2, and Notch3), CSC regulation (Notch1, Notch2, Notch4), tumor invasion and metastasis (Notch1, Notch2, Notch3, Notch4), angiogenesis (Notch3), and drug resistance (Notch1 and Notch3) [232,233]. Inactivation of Notch3, either by knockdown or by raft depletion, has been reported to sensitize the TNBC cell lines BT549 and MDA-MB-468 to gefitinib by enhancing dephosphorylation of EGFR at Y1173 and its intracellular retention, preventing its membrane localization into lipid rafts [234]. The dephosphorylation of p-EGFR (Y1173) is promoted by the Notch3-KD-induced interaction of EGFR with the protein tyrosine phosphatase H1 (PTPH1). These results suggest that reducing the EGFR cell surface expression may prevent survival signaling to enhance drug-induced cell death [234].

\subsection{Others Resistance Mechanisms}

\subsubsection{Expression of Mutant p53 (mtp53)}

Mutations of the tumor suppressor $p 53$ are well established as being associated with approximately $50 \%$ of human cancers [235-239]. A panel of TNBC cell lines has been identified to express mutant p53 (mtp53) [240], which is commonly found in TNBC [120,192]. For example, one study reported that 297 of $450(66.0 \%)$ breast cancer clinical samples contained $p 53$ mutations. More frequent $p 53$ mutations were found in TNBC $(74.8 \%)$ than in HER2-positive breast cancer (55.4\%) [241]. In addition, a separate study conducted a mutation analysis of 104 primary TNBC cases and revealed that approximately $85 \%$ had somatic $p 53$ mutations [190]. Mutations of $p 53$ often result in stabilization and overexpression of mtp53 protein [240], and overexpression of mtp53 has been associated with cancer resistance to anticancer drugs [242,243].

One plausible mechanism of EGFR overexpression in TNBC is the oncogenic mtp53 [244-246]. The activity of EGFR is regulated at multiple levels, including endosomal recycling. Endocytosis occurs to remove part of the plasma membrane including associated proteins, such as EGFR, and subsequently to form internalized membrane vesicles (early endosomes). It is a very active process, such that the entire plasma membrane is endocytosed at least once per hour [247]. Upon ligand binding, the ligand-EGFR complex is internalized by endocytosis and the early endosome forms multivesicular bodies (MVBs). MBVs now may follow three alternative pathways: (1) fuse with the lysosome to degrade their cargo; (2) go back to the plasma membrane to recycle their cargo; (3) fuse with the plasma membrane to release intralumenal vesicles into the extracellular space as exosomes [248-250]. P53 ${ }^{\mathrm{R} 273 \mathrm{H}}$ enhances recycling of EGFR to the plasma membrane in combination with integrin $\alpha 5 \beta 1$, leading to constitutive activation of EGFR-integrin signaling [251]. Mtp53 indirectly promotes the interaction of the Rab coupling protein (RCP) with $\alpha 5 \beta 1$ in the EGFR-integrin complex, while the presence of RCP has been associated with enhanced recycling of the receptor complex [251,252].

In addition, mtp53 upregulates EGFR expression through suppression and upregulation of miR-27a and miR-155, respectively $[245,246]$. Furthermore, $\mathrm{p} 53^{\mathrm{R} 273 \mathrm{H}}$ has been demonstrated to bind and suppress the promoter of miR-27a, which targets the $3^{\prime}$-UTR of EGFR [246]. In the TNBC cell line MDA-MB-468, stable expression of miR-27a mimics 
or p53 shRNA reduced the EGFR levels, with decreased colony formation in vitro and reduced the volume of xenograft tumors in vivo. The mtp53s, including R248Q, R282W, and R249S, upregulate the expression of miR-155 by relieving p63-mediated transcriptional suppression [245]. MiR-155 targeted the ZNF652, a transcriptional repression of EGFR. The ZNF652 level was consistently reduced and inversely correlated with the miR-155 level in TNBC cell lines with mtp53s.

\subsubsection{Overexpression of Anti-apoptotic Proteins}

Upregulation of anti-apoptotic proteins confers drug resistance in many cancers [253-255]. Two major anti-apoptotic protein family members tightly control both the intrinsic and extrinsic apoptosis pathways: (1) inhibitors of apoptosis proteins (IAPs) control the activity of caspases; (2) B-cell lymphoma 2 (BCL2) family proteins regulate the integrity of the mitochondrial outer membrane [253].

The anti-apoptotic members of BCL2 family proteins include BCL2, BCL2L1 (BCL-xL), BCL2-like 2 (BCL2L2, BCL-w), and myeloid cell leukemia 1 (MCL1) [256]. High expression levels of BCL2 are also associated with a poor clinical prognosis of TNBC [257]. A human tumor microarray analysis demonstrated that TNBC tumors express EGFR and co-express BCL-xL or both BCL-xL and BCL2 [258]. MCL1 has been determined to be commonly overexpressed in TNBC and is associated with a poor clinical prognosis. MCL1 is further stabilized by the overexpressed MUC1-C-mediated activation of the RAS/MEK/ERK and PI3K/AKT pathways in TNBC cells [259]. MUC1-C has additionally been determined to activate the transcription of BCL2A1 in an NF-KB-dependent manner in TNBC cells [220]. Recently, upregulation of MCL1 by elongator (ELP) complex has been reported to mediate resistance to the EGFRi erlotinib [260]. Depletion of ELP proteins, such as ELP3, ELP4, ELP5, and ELP6, sensitized TNBC cells to erlotinib, while ELP4-KD reduced expression of MCL1 in the TNBC cells in the presence of erlotinib.

\subsubsection{Contributions of Phosphatases}

In human genomes, 189 known and predicted protein phosphatases have been identified [261]. Protein phosphatases antagonize the action of protein kinases by hydrolysis of phosphate groups from target proteins. Both protein kinases and phosphatases work as key regulators of various cellular processes in normal and disease conditions. Limited number of studies reported the potential roles of protein phosphatases in EGFRi resistance in TNBC.

The protein tyrosine phosphatase non-receptor type 12 (PTPN12) has been known as a tumor suppressor in TNBC [262]. Loss of PTPN12 function is frequently found in human TNBC, leading to activation of HER2, EGFR, and PDGFR $\beta$ pathways. These results suggest a rationale to target multiple RTK pathways in TNBC [262]. In addition, the activation or compensation of PTPN12 activity may also overcome EGFRi resistance in TNBC.

The SRC homology region 2-containing protein tyrosine phosphatase (SHP2; also known as PTPN11) has been demonstrated to contribute adaptive resistance to ERK signaling inhibition in TNBC [263]. In fact, SHP2 regulates both upstream and downstream targets of RTKs, including EGFR [264]. Silencing of SHP2 by shRNA suppressed the RAS/RAF/MEK/ERK and PI3K/AKT pathways in TNBC cells. In addition, SHP2 depletion reduced expression of RTKs such as EGFR, FGFR, and MET, leading to suppression of TNBC cell proliferation, anchorage-independent growth, and mammosphere formation. Although small molecule inhibitors of SHP2 have been developed as potential therapeutics for treatment of cancers including TNBC [265-267], the efficacy of the combination of these inhibitors with EGFRi remains to be determined.

\subsubsection{Overexpression of the Heat Shock Protein 90 (HSP90)}

As a molecular chaperone, HSP90 interacts with and regulates the stability and function of over 200 client proteins, including EGFR, HER2, ALK, MET, and AKT [268-271]. Since overexpression of HSP90 has been evidenced in cancers, including breast and cer- 
vical cancers and osteosarcoma, HSP90 inhibitors (HSP90is) show therapeutic effects by suppressing multiple oncogenic pathways that are activated by its client proteins. For example, the HSP90i geldanamycin reduced the level of RPS6 in a proteasome-dependent manner [272]. Notably, RPS6-KD suppressed the proliferation of TNBC cells over time [15]. However, the efficacy of HSP90is has been limited in patients with TNBC due to both intrinsic and acquired resistance [273]. To the best of our knowledge, the potential of combined treatment of HSP90is and EGFRis has not been tested yet.

\subsubsection{Overexpression of the Estrogen Receptor Alpha (ER $\alpha)$ Variant}

A novel variant of ER $\alpha$ with a molecular weight of $36 \mathrm{kDa}$ (ER $\alpha 36$ or ER36) has been identified [274]. ER $\alpha 36$ is an alternatively spliced product of $E R \alpha 46$ that is transcribed by an alternative promoter located in the first intron of the ER $\alpha 66$ gene. ER $\alpha 36$ differs from the canonical ER $\alpha 66$, as it lacks both transcription activation domains (activation factor (AF)-1 and AF-2) but retains the DNA-binding domain and partial ligand-binding domain. ER $\alpha 36$ is involved in mammary tumor progression and resistance to drug treatment due to its ability to activate non-genomic signaling pathways such as the PI3K/AKT/mTORC1 and RAS/RAF/MEK/ERK pathways $[275,276]$. The primary location of ER $\alpha 36$ is the cytoplasm, in which it is associated with the plasma membrane to induce signal transduction [277]. A positive feedback loop of ER $\alpha 36 / E G F R$ has been identified in TNBC cells: (1) in response to estrogen, ER $\alpha 36$ physically associates with the EGFR/v-src avian sarcoma (Schmidt-Ruppin A-2) viral oncogene homolog (SRC)-SRC homology 2 domain-containingtransforming protein $\mathrm{C}$ (SHC) complex to induce phosphorylation of EGFR (Y845) and SRC (Y416); (2) EGFR-induced signaling activates the transcription of ER $\alpha 36$ through an AP-1 site in its promoter; (3) ER $\alpha 36$ prevents EGFR protein from proteasome-dependent degradation [278]. In addition, tamoxifen, a selective ER modulator [279], has been identified to enhance the stemness and metastasis of breast cancer by upregulating aldehyde dehydrogenase 1A1 (ALDH1A1) transcription in cancer cells through direct binding to and activation of ER $\alpha 36$ [280].

\subsubsection{Overexpression of Adenosine Diphosphate Ribosylation Factor (ARF)}

ARF1 has been associated with resistance to anticancer drugs in various cancers [281-285]. ARF1 is a member among 29 human ARF family members that belong to the small GTPase RAS superfamily [286,287]. GTP binding to ARF1 switches its conformation to an active one from the GDP-bound inactive form [286,287]. ARF1 is a key regulator that maintains the structure and function of the Golgi apparatus. Recently, ARF1 overexpression has been reported to promote resistance of TNBC cells to the EGFRi gefitinib [288]. Of interest, treatment of gefitinib to MDA-MB-231 cells induces increased ARF1 activity through its enhanced recruitment of AXL. ARF1 is also involved in the gefitinib-induced activation of ERK1/2, AKT, and SRC and inhibits gefitinib-induced EGFR internalization and subsequent degradation. Pharmacological inhibition of ARF1 activity potentiates gefitinibmediated anticancer activity in MDA-MB-231 cells [288] (see Section 4.2.4. Combination with ARF Exchange Activity Inhibitors (ARFis)).

\subsubsection{Dysregulation of Reactive Oxygen Species (ROS)}

ROS are important intracellular second messengers [289], and dysregulation of ROS is associated with anticancer drug resistance [290-292]. Specifically, ROS inactivate SHP2 by transient oxidation of the catalytic cysteine residue, leading to EGFR transactivation [293]. ROS also contribute to anticancer drug resistance through metabolic deregulations, including increased drug efflux, altered oncogenes or tumor suppressors, drug inactivation, epithelial-mesenchymal transition (EMT), and enhanced DNA repair [292].

\subsubsection{Expression of the Cluster of Differentiation 44 (CD44)}

CD44 is a type 1 transmembrane glycoprotein that serves as a receptor for hyaluronic acid [294-296], type 2 and IV collagens [297,298], E-selectin [299,300], fibronectin [301], 
matrix metalloproteinase 9 (MMP9) [302], and osteopontin (OPN) [303]. CD44 is also known as extracellular matrix receptor type 3 (ECM-III) [304], Hermes antigen [305], homing cell adhesion molecule (HCAM) [306], lymphocyte homing receptor [307], and phagocytic glycoprotein-1 (Pgp-1) [304]. The biology of CD44 is extremely complex, and more than 29 isoforms from a single gene consisting of 19 exons have been described in humans [308]. The shortest standard form (CD44s) is encoded by the 10 constant exons, whereas CD44 variant isoforms (CD44v) are encoded by alternatively spliced mRNAs containing the 10 constant exons and any combination of an additional 9 variant exons [309].

CD44 is involved not only in tissue tropism of stem cells and their exosomes [248,310-312], but also in tumor initiation and progression [313]. CD44 has also been established as a potential CSC marker that contributes to cancer proliferation, metastasis, and drug resistance [314]. The expression of CD44 has been correlated with the EGFR level in a variety of cancer, and their expression is positively regulated reciprocally [315]. CD44 also serves as a co-receptor for RTKs to stimulate downstream signaling pathways [309]. Recently, siRNA-based CD44s-KD has been reported to sensitize TNBC cells to cetuximab, with enhanced EGFR degradation and downregulation of p-AKT and p-ERK [316].

In addition, OPN has been identified as a biomarker for predicting responsiveness to the EGFR TKI, erlotinib, in two TNBC cell lines, MDA-MB-231 and MDA-MB-468 [317]. Engineered cell lines that overexpress OPN are more sensitive to erlotinib-mediated growth inhibition than parental cells. This might be dependent on OPN binding to integrin and provoking activation of EGFR kinase activity [318]. Consistent with these findings, OPN has been associated with poor outcomes among patients with breast cancer [319-323].

\subsubsection{Overexpression of Annexin A2 (ANAX2)}

ANXA2 is a member of the annexin family of calcium-dependent phospholipid binding proteins containing an annexin repeat [324,325]. ANXA2 has been reported to play an important role in cancer cell proliferation, metastasis, and drug resistance [326]. It also contributes to EGFR dimerization and endocytic recycling [327,328]. Recently, knockdown of ANXA2 in the TNBC cell line MDA-MB-231 has been shown to increase gefitinib sensitivity [324]. In contrast, overexpression of ANXA2 induces resistance to gefitinib in MCF7 breast cancer cells. Phosphorylation of ANXA at Y23 has been further negatively correlated with gefitinib sensitivity in TNBC cells [324].

\subsubsection{Induction of Autophagy}

Autophagy is the controlled removal of unnecessary or dysfunctional components from cells, enabling the recycling of cellular components [329]. Although autophagy has tumor-suppressive roles in normal cells by removing damaged organelles [330], it also protects cancer cells from cell death [331,332]. Mounting evidence further supports the idea that drug-induced autophagy causes resistance of cancer cells, and combined inhibition of autophagy is a plausible strategy to overcome this resistance [331-333].

EGFR TKIs and neutralizing antibodies have been shown to induce autophagy in cancer cells [334-337]. In addition, the EGFR TIK-induced accumulation of the inactive EGFR in endosomes may play a role in autophagy induced by EGFR TKIs [338]. However, more details remain to be determined to understand the function of autophagy in cancer. A recent study suggested that EGFRi-induced autophagy has a pro-survival role in TNBC cells, while combined inhibition of autophagy with EGFRis confers anticancer effects in TNBC cells [339] (see Section 4.1.11. Combination with Rho-Associated, Coiled-CoilContaining Protein Kinase (ROCK) Inhibitors).

\subsubsection{Contribution of Secretomes}

Secretomes, including cytokines, growth factors, extracellular nucleic acids, and extracellular vesicles (EVs), either from cancer cells or from stromal cells, confer drug resistance [147,340-343]. For example, HGF was identified as a resistant growth factor in a 
co-culture screening of 23 stromal cell types with 45 cancer cell lines against 35 anticancer drugs [344]. NRG1 was also shown to drive the resistance of cancer cells to PKIs [147].

IL-25 (IL-17E), a member of the IL-17 family, binds and activates the IL-17RA/IL17RB heterodimer through direct biding to IL-17B [345,346]. The IL-25/IL17RB pathway has contributed to the resistance of TNBC to EGFRis [347]. IL-25 activates the EGFR pathway in TNBC cells with resistance to the EGFRi gefitinib. IL-25 also activates PYK2 and SRC, leading to the SRC-dependent activation and nuclear translocation of p-STAT3 and p-EGFR [347].

EVs collectively refer to the membranous vesicles released from living cells, such as exosomes, microvesicles, and apoptotic bodies [248,348]. Tumor-derived EVs have been demonstrated to mediate drug resistance via multiple mechanisms. For example, EVs can: (1) reduce the accumulation of anticancer drugs inside cancer cells; (2) traffic functional cargo, activating pro-survival or anti-apoptotic signals in cancer cells; (3) suppress immune reactions in cancer microenvironments; (4) enhance EMT and induce CSC-like properties (reviewed in [349]). In addition, tumor-derived EVs from patients' body fluids could be used for liquid biopsy to diagnose disease status and drug sensitivity [350]. More importantly, tumor-derived EVs may serve as therapeutic targets, drug delivery vehicles, or therapeutics themselves [350].

Taken together, the understanding of the complicated interplay of autocrine and paracrine signaling occurring between tumor cells and normal cells in their associated microenvironment will provide valuable information to develop more effective clinical strategies to combat drug resistance.

\section{Combination Strategy for Overcoming EGFRi Resistance in TNBC}

Combination therapies have been proven to overcome resistance to EGFR TKIs in clinical settings for NSCLC [351-363]. A variety of combination strategies has been explored preclinically in TNBC cells (Table 3). Among the 73 publications analyzed, 60 reported the preclinical results of combinatorial therapies. Combination approaches were explored as early as 2004 with small-molecule EGFR TKIs and the anti-EGFR antibodies [364]. with a growing number of small-molecule PKIs, combination strategies have been applied to target multiple cellular pathways. We briefly summarize EGFR-potentiating strategies according to the target categories.

\subsection{PKIs}

Protein kinases play pivotal roles in the pathogenesis of various diseases, including cancer. A plethora of PKIs targeting such kinases are under development to be used for therapeutic interventions. As of 23 December 2020, sixty-two PKIs have already been approved by the US FDA [49]. In addition, three more PKIs, including tepotinib (Merck), trilaciclib (G1 Therapeutics), and umbralisib (TG Therapeutics), have been approved by the US FDA as on 2 March 2021(PKIDB: a curated, annotated, and updated database of protein kinase inhibitors in clinical trials, https://www.icoa.fr/pkidb/, accessed on 2 March 2021) $[457,458]$. The FDA-approved PKIs that have been tested for efficacy in combination treatment with EGFRis in TNBC cells are shown in Table 4. Notably, most anti-EGFR therapeutics used in combination are also FDA-approved EGFR TKIs or antibodies. 
Table 3. Potentiating EGFR inhibition by combination strategies.

\begin{tabular}{|c|c|c|c|c|c|c|c|c|}
\hline \multirow[b]{2}{*}{ Year } & \multirow[b]{2}{*}{ EGFRi } & \multicolumn{2}{|c|}{ Combination Drug } & \multirow[b]{2}{*}{ TNBC Cells } & \multirow[b]{2}{*}{$\begin{array}{l}\text { Molecules Affected by } \\
\text { Combination }\end{array}$} & \multicolumn{2}{|r|}{ Combination Effects } & \multirow[b]{2}{*}{ Ref } \\
\hline & & Name & $\begin{array}{l}\text { Known Target } \\
\left(\mathrm{IC}_{50}, \mathrm{nM}\right)\end{array}$ & & & Level & Effects & \\
\hline 2004 & Gefitinib & Cetuximab & EGFR & MDA-MB-468 & - & Cell & - Reduced cell proliferation & [364] \\
\hline 2008 & Cetuximab & Cisplatin & DNA & MDA-MB-468 & $\begin{array}{l}\text { Down: EGFR } \\
\text { Up: BRCA1 }\end{array}$ & Cell & $\begin{array}{l}\text { - Synergistically induced apoptotic cell death } \\
\text { - Depleted EGFR protein }\end{array}$ & [365] \\
\hline 2009 & Gefitinib & $\begin{array}{l}\text { Carboplatin + } \\
\text { Docetaxel }\end{array}$ & $\begin{array}{c}\text { DNA } \\
\text { Microtubule }\end{array}$ & $\begin{array}{c}\text { BT20 } \\
\text { HCC9137 }\end{array}$ & - & Cell & $\begin{array}{l}\text { - Reduced cell viability } \\
\text { - Induced } \mathrm{G}_{2} / \mathrm{M} \text { cell cycle arrest }\end{array}$ & [366] \\
\hline 2011 & Erlotinib & Gemcitabine & Antimetabolite & $\begin{array}{c}\text { BT-549 } \\
\text { MBA-MB-231 } \\
\text { MDA-MB-468 }\end{array}$ & - & Cell & - Synergistically reduced cell viability & [11] \\
\hline \multirow[t]{2}{*}{2011} & \multirow[t]{2}{*}{ Lapatinib } & \multirow[t]{2}{*}{$\begin{array}{l}\text { Sirolimus } \\
\text { (Rapamycin) }\end{array}$} & \multirow[t]{2}{*}{$\operatorname{mTOR}(\sim 0.1)[367]$} & $\begin{array}{l}\text { MDA-MB-231 } \\
\text { MDA-MB-468 }\end{array}$ & $\begin{array}{l}\text { Down: p-AKT (S473), p-EGFR } \\
\text { (Y1173), p-ERK1/2 (T202/Y204), p-S6 } \\
\text { (S235/236) }\end{array}$ & Cell & $\begin{array}{l}\text { - Reduced survival of MDA-MB-231 and } \\
\text { MDA-MB-468 but only induced apoptosis of } \\
\text { MDA-MB-468 with a concomitant increase of } \\
\text { cleaved caspase } 3 \\
\text { - Differentially affected p-eIF4E (S209) levels in } \\
\text { MDA-MB-468 (down) versus in MDA-MB-231 (up) }\end{array}$ & \multirow[t]{2}{*}{368} \\
\hline & & & & $\begin{array}{l}\text { MDA-MB-231 } \\
\text { MDA-MB-468 }\end{array}$ & $\begin{array}{l}\text { Down: Ki67 } \\
\text { Up: Caspase-3 }\end{array}$ & Xeno $^{1}$ & $\begin{array}{l}\text { - Inhibited growth of xenograft tumors of both cells } \\
\text { with decreased Ki67 in both tumors and increased } \\
\text { apoptosis in MDA-MB-468 tumor }\end{array}$ & \\
\hline 2011 & Cetuximab & IL-2 or IL-15 & NK cells & $\begin{array}{c}\text { IIB-BR-G } \\
\text { IIB-BR-G MT }\end{array}$ & $\begin{array}{l}\text { Up: IFN- } \gamma \text { from NK cells in a } \\
\text { co-culture }\end{array}$ & Cell & - Increased ADCC by NK cells & [369] \\
\hline 2012 & Cetuximab & IL-2 or IL-15 & NK cells & $\begin{array}{l}\text { IIB-BR-G } \\
\text { IIB-BR-G MT }\end{array}$ & $\begin{array}{l}\text { Up: CD107a in NK cells in a } \\
\text { co-culture }\end{array}$ & $\begin{array}{l}\text { Cell } \\
\text { Ex vivo } \\
\text { Xeno }\end{array}$ & $\begin{array}{l}\text { - Increased ADCC by NK cells } \\
\text { - Reduced tumor volume in xenografts with an } \\
\text { increase in the number of infiltrating NK cells in the } \\
\text { tumors } \\
\text { - No effect on tumor proliferation in xenografts }\end{array}$ & [370] \\
\hline \multirow[t]{2}{*}{2012} & \multirow[t]{2}{*}{ Lapatinib } & \multirow{2}{*}{$\begin{array}{l}\text { Veliparib } \\
\text { (NSC 737664, } \\
\text { ABT-888) }\end{array}$} & \multirow[t]{2}{*}{$\begin{array}{l}\text { PARP2 (2.9), PARP1 } \\
\text { (5.2) [371] }\end{array}$} & $\begin{array}{l}\text { MDA-MB-231 } \\
\text { MDA-MB-453 } \\
\text { MDA-MB-468 }\end{array}$ & $\begin{array}{l}\text { Down: nuclear BRCA1, nuclear EGFR } \\
\text { Up: cleaved caspase-3, cleaved } \\
\text { caspase-9, } \gamma \text {-H2AX }\end{array}$ & Cell & $\begin{array}{l}\text { - Reduced colony formation } \\
\text { - Induced apoptosis }\end{array}$ & \multirow[t]{2}{*}{372} \\
\hline & & & & MDA-MB-231 & - & Xeno & - Reduced tumor growth & \\
\hline 2012 & Gefitinib & CAT-SKL $^{2}$ & - & $\begin{array}{l}\text { MDA-MB-468 } \\
\text { SUM149PT }\end{array}$ & Down: p-EGFR (Y1068) & Cell & - Reduced cell proliferation & [373] \\
\hline
\end{tabular}


Table 3. Cont.

\begin{tabular}{|c|c|c|c|c|c|c|c|c|}
\hline \multirow[b]{2}{*}{ Year } & \multirow[b]{2}{*}{ EGFRi } & \multicolumn{2}{|c|}{ Combination Drug } & \multirow[b]{2}{*}{ TNBC Cells } & \multirow{2}{*}{$\begin{array}{l}\text { Molecules Affected by } \\
\text { Combination }\end{array}$} & \multicolumn{2}{|r|}{ Combination Effects } & \multirow[b]{2}{*}{ Ref } \\
\hline & & Name & $\begin{array}{l}\text { Known Target } \\
\left(\mathrm{IC}_{50}, \mathrm{nM}\right)\end{array}$ & & & Level & Effects & \\
\hline 2013 & Cetuximab & Dasatinib + Cisplatin & $\begin{array}{l}\text { Dasatinib: ABL1 } \\
(0.6), \text { SRC (0.8) [374]; } \\
\text { KITT D816V (37), KIT }^{\text {(79) [375] }}\end{array}$ & $\begin{array}{c}\text { BT20 } \\
\text { BT549 } \\
\text { MDA-MB-231 } \\
\text { SUM102PT } \\
\text { SUM149PT } \\
\text { SUM229PE }\end{array}$ & $\begin{array}{c}\text { Down: p-AKT (S473), p-EGFR (Y845), } \\
\text { p-EGFR (Y1068), p-EGFR (Y1173), } \\
\text { p-ERK (T202/Y204) } \\
\text { Up: cleaved caspase-9 }\end{array}$ & Cell & $\begin{array}{l}\text { - Induced apoptosis } \\
\text { - Reduced cell growth and migration }\end{array}$ & [376] \\
\hline 2013 & Gefitinib & PI-103 & $\begin{array}{l}\text { DNA-PK (2), PI3K } \alpha \\
\text { (8), mTORC1 (20), } \\
\text { PI3K } \delta \text { (48), mTORC2 } \\
\text { (83), PI3K } \beta(88), \\
\text { PI3K } \gamma(150)[377]\end{array}$ & $\begin{array}{c}\text { MDA-MB- } \\
\text { 468SUM149PT }\end{array}$ & $\begin{array}{c}\text { Down: p-AKT (S473), BCL2, p-ERK } \\
\text { (Y204.Y187), MCL1, XIAP } \\
\text { Up: caspase-3/7 }\end{array}$ & Cell & $\begin{array}{l}\text { - Reduced cell viability and induced apoptosis in the } \\
\text { BL subtype (MDA-MB-468 and SUM149PT) but not } \\
\text { in the MSL subtype (HS578T and MDA-MB-231) }\end{array}$ & [378] \\
\hline \multirow[t]{2}{*}{2013} & \multirow[t]{2}{*}{ Lapatinib } & \multirow{2}{*}{$\begin{array}{c}\text { Bortezomib } \\
\text { (Velcade }{ }^{\circledR}, \text { PS-341, } \\
\text { LDP-341, LM341) }\end{array}$} & \multirow{2}{*}{$\begin{array}{l}20 S \text { proteasome }(\mathrm{Ki}= \\
\text { 0.6) }[379]\end{array}$} & $\begin{array}{c}\text { HS578T } \\
\text { MDA-MB-231 }\end{array}$ & $\begin{array}{l}\text { Down: BCL2 } \\
\text { Up: BAX, Cleaved Caspase-3, } \\
\text { Cleaved PARP }\end{array}$ & Cell & - Reduced colony formation & \multirow[t]{2}{*}[380]{} \\
\hline & & & & MDA-MB-231 & $\begin{array}{l}\text { Down: P65 } \\
\text { Up: BAX }\end{array}$ & Xeno & - Reduced tumor growth & \\
\hline 2013 & Panitumumab & mAb111 & EGFR & HCC70 & - & Xeno & - Reduced tumor volume & [381] \\
\hline 2014 & Erlotinib & PHA-665752 & $\begin{array}{l}\text { MET (9), RON (68), } \\
\text { FLK1 (200) [382] }\end{array}$ & $\begin{array}{c}\text { BT20 } \\
\text { CRC029 } \\
\text { MDA-MB-468 }\end{array}$ & - & Cell & - Reduced cell viability & [150] \\
\hline 2014 & Gefitinib & $\begin{array}{c}\text { Tepotinib } \\
\text { (Tepmetko }^{\circledR} \\
\text { EMD1214063) }^{2}\end{array}$ & MET (4) [383] & MDA-MB-468 & $\begin{array}{l}\text { Down: p-AKT (T308), p-ERK } \\
\text { (T202/Y204), p-RPS6 (S235/236) }\end{array}$ & Cell & - Reduced growth and colony formation & [153] \\
\hline \multirow{2}{*}{2014} & \multirow{2}{*}{$\begin{array}{l}\text { Duligotuzumab } \\
\text { (MEHD7945A) }\end{array}$} & $\begin{array}{c}\text { Ipatasertib } \\
\text { (GDC-0068, RG7440) }\end{array}$ & $\begin{array}{l}\text { AKT1 (5), AKT3 (8), } \\
\text { AKT2 (18) [384] }\end{array}$ & \multirow{2}{*}{$\begin{array}{c}\text { HCC70 } \\
\text { MDA-MB-468 }\end{array}$} & $\begin{array}{l}\text { Down: p-EGFR (Y1068), p-ERK } \\
\text { (T202/Y204), p-HER3 (Y1289), } \\
\text { p-RPS6 (S240/244)Up: p-AKT (T308) }\end{array}$ & Cell & - Reduced cell proliferation & \multirow{2}{*}{ [159] } \\
\hline & & $\begin{array}{c}\text { Pictilisib } \\
\text { (GDC-0941, RG7321) }\end{array}$ & $\begin{array}{c}\text { PI3K } \alpha(3), \text { PI3K } \delta(3), \\
\text { PI3K } \beta(33), \text { PI3K } \gamma \\
\text { (75), mTOR }(\mathrm{Ki}= \\
0.58 \mu \mathrm{M})[385]\end{array}$ & & $\begin{array}{l}\text { Down: p-AKT (S473), p-AKT (T308), } \\
\text { p-EGFR (Y1068), p-ERK (T202/Y204), } \\
\text { p-HER3 (Y1289), p-RPS6 (S240/244) }\end{array}$ & $\begin{array}{l}\text { Xeno } \\
\text { PDX }\end{array}$ & $\begin{array}{l}\text { - Reduced tumor growth of HCC70 and PDX } \\
\text { - Reduced Ki67 index in xenograft tumor }\end{array}$ & \\
\hline 2014 & Gefitinib & Temsirolimus & mTOR (1760) [386] & $\begin{array}{c}\text { BT20 } \\
\text { MDA-MB-468 } \\
\text { MDA-MB-231 }\end{array}$ & Down: p-eIF4B (S422) & Cell & $\begin{array}{l}\text { - Reduced cell growth, colony formation, and cell } \\
\text { viability } \\
\text { - Reduced cap-dependent translation }\end{array}$ & [387] \\
\hline
\end{tabular}


Table 3. Cont.

\begin{tabular}{|c|c|c|c|c|c|c|c|c|}
\hline \multirow[b]{2}{*}{ Year } & \multirow[b]{2}{*}{ EGFRi } & \multicolumn{2}{|c|}{ Combination Drug } & \multirow[b]{2}{*}{ TNBC Cells } & \multirow[b]{2}{*}{$\begin{array}{l}\text { Molecules Affected by } \\
\text { Combination }\end{array}$} & \multicolumn{2}{|r|}{ Combination Effects } & \multirow[b]{2}{*}{ Ref } \\
\hline & & Name & $\begin{array}{c}\text { Known Target } \\
\left(\mathrm{IC}_{50}, \mathrm{nM}\right)\end{array}$ & & & Level & Effects & \\
\hline \multirow[b]{2}{*}{2014} & \multirow[b]{2}{*}{ Gefitinib } & \multirow{2}{*}{$\begin{array}{c}\text { SKI II } \\
\text { (SphK-I2) }\end{array}$} & \multirow[b]{2}{*}{ SPHK (500) [388] } & HS578T & \multirow{2}{*}{-} & Cell & - Reduced cell proliferation & \multirow[b]{2}{*}{ [389] } \\
\hline & & & & $\begin{array}{l}\text { MDA-MB-231 } \\
\text { MDA-MB-436 } \\
\text { MDA-MB-468 }\end{array}$ & & Xeno & $\begin{array}{l}\text { - Reduced the growth of MDA-BM- } 468 \text { xenograft } \\
\text { tumor }\end{array}$ & \\
\hline 2015 & Gefitinib & SU11274 & MET (10) [390] & $\begin{array}{c}\text { HS578T } \\
\text { MDA-MB-231 }\end{array}$ & $\begin{array}{c}\text { Down: p-AKT (S473), RPS6, p-RPS6 } \\
\text { (S235/236) }\end{array}$ & Cell & $\begin{array}{l}\text { - Reduced cell viability and colony formation } \\
\text { - No synergistic effect on cell cycle distribution }\end{array}$ & [15] \\
\hline 2015 & Gefitinib & $\begin{array}{l}\text { Selumetinib } \\
\text { (AZD6244) }\end{array}$ & $\begin{array}{l}\text { MEK1 }(14)[391] \\
\text { MEK2 }\left(K_{D}=530\right) \\
{[392]}\end{array}$ & $\begin{array}{c}\text { HCC70 } \\
\text { MDA-MB-231 } \\
\text { MDA-MB-468 } \\
\text { SUM149PT } \\
\text { SUM159PT }\end{array}$ & $\begin{array}{l}\text { Down: p-AKT (S473), p-ERK } \\
\text { (T202/Y204) }\end{array}$ & Cell & $\begin{array}{l}\text { - Induced } \mathrm{G}_{0} / \mathrm{G}_{1} \text { cell cycle arrest and apoptosis in } \\
\text { SUM149PT }\end{array}$ & [393] \\
\hline 2015 & Gefitinib & Brefeldin A & $\begin{array}{c}\text { ARF purified from } \\
\text { bovine brain }(2 \mu \mathrm{M}) \\
\text { [394], ARF1 }(10 \mu \mathrm{M}) \\
\text { [395] }\end{array}$ & MDA-MB-231 & $\begin{array}{l}\text { Down: p-AKT, EGFR, p-ERK1/2, } \\
\text { HER2, p-SRC }\end{array}$ & Cell & - Induced cell death & [288] \\
\hline \multirow[t]{2}{*}{2015} & \multirow[t]{2}{*}{ Lapatinib } & \multirow[t]{2}{*}{$\begin{array}{l}\text { Imatinib } \\
\left(\text { Gleevec }^{\circledR}\right)\end{array}$} & \multirow{2}{*}{$\begin{array}{l}\text { PDGFR (100), KIT } \\
\text { (100), ABL1 (600) } \\
\text { [396] }\end{array}$} & $\begin{array}{c}\text { HCC1806 } \\
\text { MDA-MB-231 } \\
\text { SUM159PT }\end{array}$ & Down: HOTAIR, $\beta$-catenin, MYC & Cell & - Reduced cell viability & \multirow[t]{2}{*}{397} \\
\hline & & & & MDA-MB-231 & - & Xeno & - Reduced tumor growth & \\
\hline \multirow[t]{2}{*}{2015} & \multirow[t]{2}{*}{ Gefitinib } & \multirow[t]{2}{*}{ Ad-wtp53 } & \multirow[t]{2}{*}{-} & \multirow[t]{2}{*}{ MDA-MB-468 } & \multirow[t]{2}{*}{$\begin{array}{c}\text { Down: p-AKT (S473) } \\
\text { Up: cleaved caspase-3, caspase-9 }\end{array}$} & Cell & $\begin{array}{l}\text { - Inhibited cell growth and colony formation } \\
\text { - Induced apoptosis and } G_{2} / M \text { arrest }\end{array}$ & \multirow[t]{2}{*}[398]{} \\
\hline & & & & & & Xeno & - Reduced tumor growth & \\
\hline 2016 & Erlotinib & $\begin{array}{l}\text { Glesatinib } \\
\text { (MGCD265) }\end{array}$ & $\begin{array}{c}\text { DDR2 (1.9), AXL } \\
\text { (5.3), MERTK (7.8), } \\
\text { PDGFR } \alpha \text { (14), } \\
\text { VEGFR3 (FLT4) (23), } \\
\text { FLT3 (31), MET (46), } \\
\text { VEGFR2 (KDR) (66), } \\
\text { PDGFR } \beta \text { (69) [399] }\end{array}$ & Primary TNBC & $\begin{array}{c}\text { Down: p-ERK1/2 (T202/Y204), } \\
\text { p-RPS6 (S240/244) }\end{array}$ & $\mathrm{PDX}^{3}$ & - Reduced tumor growth & [400] \\
\hline
\end{tabular}


Table 3. Cont

\begin{tabular}{|c|c|c|c|c|c|c|c|c|}
\hline \multirow[b]{2}{*}{ Year } & \multirow[b]{2}{*}{ EGFRi } & \multicolumn{2}{|c|}{ Combination Drug } & \multirow[b]{2}{*}{ TNBC Cells } & \multirow{2}{*}{$\begin{array}{l}\text { Molecules Affected by } \\
\text { Combination }\end{array}$} & \multicolumn{2}{|r|}{ Combination Effects } & \multirow[b]{2}{*}{ Ref } \\
\hline & & Name & $\begin{array}{c}\text { Known Target } \\
\left(\mathrm{IC}_{50}, \mathrm{nM}\right)\end{array}$ & & & Level & Effects & \\
\hline & & $\begin{array}{c}\text { Crizotinib } \\
(\text { PF-02341066) }\end{array}$ & 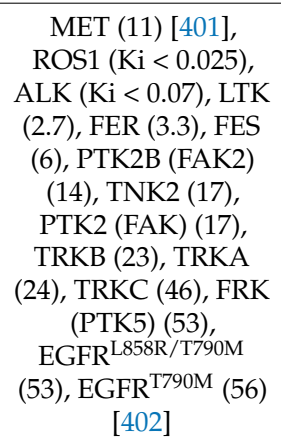 & & & & & \\
\hline 2016 & $\begin{array}{l}\text { Gefitinib or } \\
\text { Erlotinib }\end{array}$ & $\begin{array}{l}\text { Cetuximab or } \\
\text { Panitumumab }\end{array}$ & EGFR & $\begin{array}{l}\text { MDA-MB-468 } \\
\text { SUM1315MO2 }\end{array}$ & - & Cell & - Induced $G_{1}$ cell cycle arrest & [403] \\
\hline \multirow[b]{2}{*}{2016} & \multirow[b]{2}{*}{ Gefitinib } & \multirow[b]{2}{*}{ GSK269962A } & \multirow{2}{*}{$\begin{array}{c}\text { ROCK1 (1.6), } \\
\text { ROCK2 (4), MSK1 } \\
\text { (49), RSK1 (132) [404] }\end{array}$} & HCC1806 & - & Xeno & - Reduced tumor growth & \multirow[b]{2}{*}{405} \\
\hline & & & & MDA-MB-231 & $\begin{array}{l}\text { Down: CDK2, Cyclin A, p27, p-RB } \\
\text { (S807/811) }\end{array}$ & Cell & $\begin{array}{l}\text { - Inhibited colony formation } \\
\text { - Reduced S phase and increased G2 cell cycle arrest }\end{array}$ & \\
\hline \multirow{2}{*}{2016} & \multirow{2}{*}{ Cetuximab } & \multirow{2}{*}{ Ixabepilone } & \multirow{2}{*}{ Microtubule } & \multirow{2}{*}{ SUM159PT } & Down: LC3B, p62 & Cell & - Reduced mammosphere formation efficiency & \multirow{2}{*}{406} \\
\hline & & & & & - & Xeno & - Reduced tumor growth & \\
\hline 2017 & $\begin{array}{l}\text { Cetuximab } \\
\text { or panitu- } \\
\text { mumab }\end{array}$ & $\begin{array}{l}\text { Cisplatin or } \\
\text { epirubicin }\end{array}$ & & SUM1315MO2 & - & Cell & $\begin{array}{l}\text { - Induced } \mathrm{G}_{1} \text { cell cycle arrest compared to a } \\
\text { DNA-damaging agent alone that induced } \mathrm{G} 2 \text { cell } \\
\text { cycle arrest }\end{array}$ & [407] \\
\hline \multirow[t]{2}{*}{2017} & \multirow[t]{2}{*}{ Lapatinib } & \multirow[t]{2}{*}{$\begin{array}{c}\text { JNK-IN-8 } \\
\text { (JNK Inhibitor XVI) }\end{array}$} & \multirow{2}{*}{ 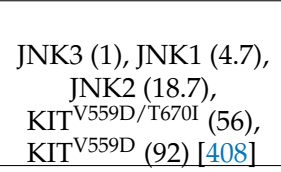 } & $\begin{array}{c}\text { HCC1569 } \\
\text { MDA-MB-231 } \\
\text { MDA-MB-436 } \\
\end{array}$ & - & Cell & - Induced apoptosis & \multirow[t]{2}{*}{231} \\
\hline & & & & MDA-MB-231 & - & Xeno & - Reduced tumor growth & \\
\hline \multirow[t]{2}{*}{2017} & \multirow[t]{2}{*}{$\begin{array}{l}\text { Erlotinib or } \\
\text { gefitinib }\end{array}$} & PF573228 & FAK1 (4) [409] & $\begin{array}{c}\text { BT20 } \\
\text { HCC } 38 \\
\text { HCC1143 } \\
\text { HCC1937 } \\
\text { MDA-MB-468 }\end{array}$ & $\begin{array}{l}\text { Down: p-AKT (S473), p-AKT (T308), } \\
\text { HER3, p-S6K (T389), p-STAT3 (Y705) }\end{array}$ & Cell & - Reduced cell proliferation and colony formation & \multirow[t]{2}{*}{ [410] } \\
\hline & & PF431396 & $\begin{array}{l}\text { FAK1 (2), PYK2 (11) } \\
\text { [411] }\end{array}$ & MDA-MB-468 & - & Xeno & - Reduced tumor growth & \\
\hline
\end{tabular}


Table 3. Cont.

\begin{tabular}{|c|c|c|c|c|c|c|c|c|}
\hline \multirow[b]{2}{*}{ Year } & \multirow[b]{2}{*}{ EGFRi } & \multicolumn{2}{|c|}{ Combination Drug } & \multirow[b]{2}{*}{ TNBC Cells } & \multirow[b]{2}{*}{$\begin{array}{l}\text { Molecules Affected by } \\
\text { Combination }\end{array}$} & \multicolumn{2}{|r|}{ Combination Effects } & \multirow[b]{2}{*}{ Ref } \\
\hline & & Name & $\begin{array}{c}\text { Known Target } \\
\left(\mathrm{IC}_{50}, \mathrm{nM}\right)\end{array}$ & & & Level & Effects & \\
\hline \multirow{3}{*}{2017} & \multirow{3}{*}{ Gefitinib } & \multirow{3}{*}{$\begin{array}{c}\text { Fingolimod } \\
\left(\text { Gilenya }^{\circledR}, \text { FTY720) }\right.\end{array}$} & \multirow{3}{*}{ S1PR (0.033) [412] } & $\begin{array}{c}\text { HCC1806 } \\
\text { MDA-MB-468 }\end{array}$ & Down: CD44, IGFBP3 & Cell & - Reduced cell proliferation & \multirow{3}{*}{ [413] } \\
\hline & & & & $\begin{array}{c}\text { HCC1806 } \\
\text { MDA-MB-468 }\end{array}$ & \multirow[t]{2}{*}{$\begin{array}{l}\text { Down: p-EGFR (Y1068), Ki67 } \\
\text { Up: cleaved caspase-3 }\end{array}$} & Xeno & \multirow[t]{2}{*}{$\begin{array}{l}\text { - Inhibited tumor growth and increased mouse } \\
\text { survival }\end{array}$} & \\
\hline & & & & $4 \mathrm{~T} 1$ & & Syn ${ }^{4}$ & & \\
\hline 2017 & Gefitinib & Bafilomycin A1 & $\begin{array}{c}\text { H+-ATPase (0.44) } \\
{[416]}\end{array}$ & MDA-MB-468 & Up: cleaved caspase-3 & Xeno & - Reduced tumor growth & {$[415$} \\
\hline 2017 & Erlotinib & $\frac{\text { CAT-SKL }}{\text { (-)-epicatechin }}$ & - & $\begin{array}{c}\text { HCC70 } \\
\text { MDA-MB-468 }\end{array}$ & - & Cell & - Reduced cell viability & [417] \\
\hline 2018 & Cetuximab & YM-1 & $\begin{array}{c}\text { BAG3-HSP70 } \\
\text { interaction }(4.8 \mu \mathrm{M}) \\
{[418]}\end{array}$ & $\begin{array}{c}\text { BT549 } \\
\text { MDA-MB-468 }\end{array}$ & - & Cell & - Reduced cell viability & [419] \\
\hline 2018 & Gefitinib & MK-2206 & $\begin{array}{l}\text { AKT1 (5), AKT2 (12), } \\
\text { AKT3 (65) [420] }\end{array}$ & $\begin{array}{c}\text { HS578T } \\
\text { MDA-MB-231 }\end{array}$ & $\begin{array}{c}\text { Down: p-mTOR (S2448), RPS6, } \\
\text { p-RPS6 (S235/236), p-RPAS40 (T246), } \\
\text { XIAP }\end{array}$ & Cell & $\begin{array}{l}\text { - Reduced cell viability, proliferation, and colony } \\
\text { formation } \\
\text { - No synergistic effect on cell cycle distribution }\end{array}$ & [421] \\
\hline 2018 & Lapatinib & $\begin{array}{c}\text { Foretinib } \\
(\text { GSK1363089, XL880) }\end{array}$ & $\begin{array}{c}\text { MET (0.4), } \\
\text { VEGFR2/KDR (0.86), } \\
\text { TIE2 (1.1), } \\
\text { VEGFR3/FLT4 (2.8), } \\
\text { RON (3), FLT3 (3.6), } \\
\text { PDGFR } \alpha \text { (3.6), KIT } \\
\text { (3.6), VEGFR1/FLT1 } \\
\text { (6.8), PDGFR } \beta \text { (9.6) } \\
\text { [422] }\end{array}$ & $\begin{array}{c}\text { BT549 } \\
\text { MDA-MB-231 }\end{array}$ & Down: p-AKT (S473) & Cell & - Reduced cell viability and migration & [423] \\
\hline \multirow[t]{2}{*}{2018} & \multirow[t]{2}{*}{ Gefitinib } & $\begin{array}{c}\text { Tepotinib } \\
\text { (Tepmetko }^{\circledR} \\
\text { EMD1214063) }\end{array}$ & MET (4) [383] & \multirow[t]{2}{*}{ MDA-MB-468 } & \multirow{2}{*}{$\begin{array}{c}\text { Down: p-ERK1/2 (T202/Y204), } \\
\text { p-STAT3 (Y705) }\end{array}$} & \multirow[t]{2}{*}{ Cell } & \multirow[t]{2}{*}{ - Reduced cell viability and colony formation } & \multirow[t]{2}{*}{ [424] } \\
\hline & & PF431396 & $\begin{array}{l}\text { FAK1 (2), PYK2 (11) } \\
\text { [411] }\end{array}$ & & & & & \\
\hline
\end{tabular}


Table 3. Cont.

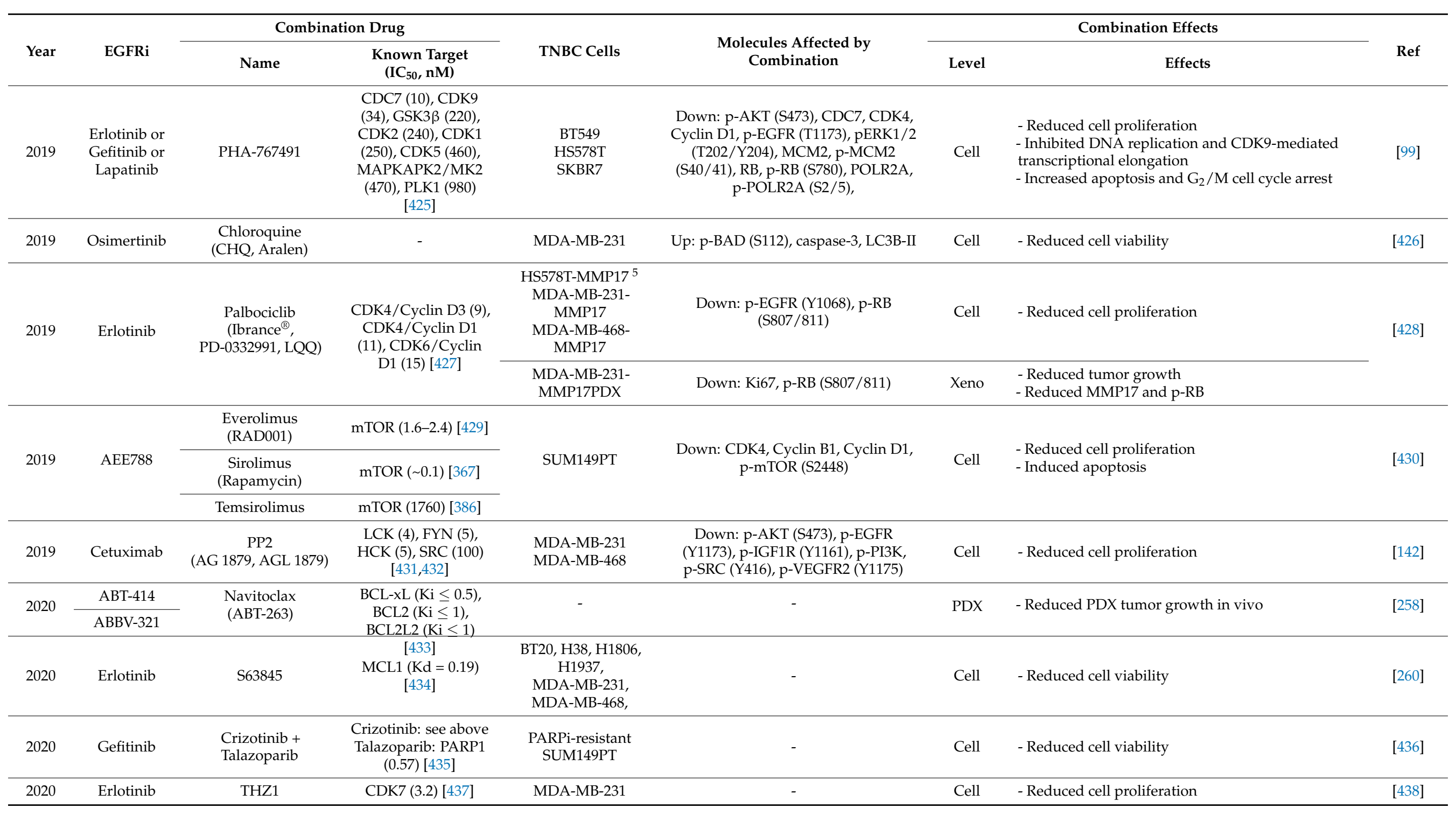


Table 3. Cont.

\begin{tabular}{|c|c|c|c|c|c|c|c|c|}
\hline \multirow[b]{2}{*}{ Year } & \multirow[b]{2}{*}{ EGFRi } & \multicolumn{2}{|c|}{ Combination Drug } & \multirow[b]{2}{*}{ TNBC Cells } & \multirow{2}{*}{$\begin{array}{l}\text { Molecules Affected by } \\
\text { Combination }\end{array}$} & \multicolumn{2}{|r|}{ Combination Effects } & \multirow[b]{2}{*}{ Ref } \\
\hline & & Name & $\begin{array}{l}\text { Known Target } \\
\left(\mathrm{IC}_{50}, \mathrm{nM}\right)\end{array}$ & & & Level & Effects & \\
\hline 2020 & Cetuximab & IL-15 & NK cells & IIB-BR-G & $\begin{array}{l}\text { Up: CD25 and CD69 in NK cells; } \\
\text { CD83 and CD86 in DCs; IFN- } \gamma \text { and } \\
\text { TNF- } \alpha \text { in co-culture supernatant }\end{array}$ & Cell & $\begin{array}{l}\text { - Activated NK cell tumor killing } \\
\text { - Stimulated maturation of DCs }\end{array}$ & [439] \\
\hline 2020 & Cetuximab & Dasatinib & $\begin{array}{c}\text { ABL1 (0.6), SRC (0.8) } \\
{[374]} \\
\operatorname{KIT}^{\mathrm{D} 816 \mathrm{~V}}(37), \mathrm{KIT} \\
\text { (79) [375] }\end{array}$ & MDA-MB-468 & Down: SRC, p-SRC (Y416) & Xeno & - Reduced tumor volume & [440] \\
\hline \multirow{2}{*}{2020} & \multirow{2}{*}{ Gefitinib } & \multirow{2}{*}{ GSK269962A } & \multirow{2}{*}{$\begin{array}{c}\text { ROCK1 (1.6), } \\
\text { ROCK2 (4), MSK1 } \\
\text { (49), RSK1 (132) [404] }\end{array}$} & MDA-MB-231 & $\begin{array}{l}\text { Down: p-RPS6 (S235/236) } \\
\text { Up: p-AMPK (T172) }\end{array}$ & \multirow{2}{*}{ Cell } & \multirow{2}{*}{ - Increased accumulation of autophagic vacuoles } & \multirow{2}{*}{339} \\
\hline & & & & CAL120HCC1806HS578T & $\begin{array}{l}\text { Down: p-RPS6 (S235/236) } \\
\text { Up: LC3-II }\end{array}$ & & & \\
\hline \multirow[t]{2}{*}{2020} & \multirow[t]{2}{*}{ Afatinib } & \multirow[t]{2}{*}{ Dasatinib } & \multirow{2}{*}{$\begin{array}{c}\text { ABL1 (0.6), SRC (0.8) } \\
{[374]} \\
\operatorname{KIT}^{\mathrm{D} 816 \mathrm{~V}}(37), \mathrm{KIT} \\
\text { (79) [375] }\end{array}$} & $\begin{array}{c}\text { BT20 } \\
\text { HCC1937 } \\
\text { HDQP1 }\end{array}$ & $\begin{array}{l}\text { Down: p-EGFR (Y1068), p-SFKs } \\
\text { (Y416), p-SRC (Y527)Up: cleaved } \\
\text { caspase-7, p27 }\end{array}$ & Cell & $\begin{array}{l}\text { - Reduced cell proliferation- Induced } \mathrm{G}_{1} \text { cell cycle } \\
\text { arrest }\end{array}$ & \multirow[t]{2}{*}{441} \\
\hline & & & & HCC1806 & Down: CDC42, p-EGFR (Y1068) & Xeno & - Reduced tumor growth & \\
\hline 2020 & Afatinib & $\begin{array}{l}\text { Dasatinib + } \\
\text { Trametinib } \\
\text { (Mekinist }^{\circledR}, \\
\text { JTP-74057, } \\
\text { GSK1120212) }\end{array}$ & $\begin{array}{l}\text { Dasatinib: see above } \\
\text { Trametinib: MEK1 } \\
\text { (0.92), MEK2 (1.8) } \\
\text { [443] }\end{array}$ & $\begin{array}{c}\text { BT20 } \\
\text { MDA-MB-468 }\end{array}$ & - & Cell & - Reduced cell proliferation & [444] \\
\hline \multirow{2}{*}{2020} & \multirow{2}{*}{\multicolumn{2}{|c|}{$\begin{array}{l}\text { Bispecific antibody for EGFR } \\
\text { (cetuximab) and HER3 (IgG 3-43) }\end{array}$}} & \multirow{2}{*}{$\begin{array}{c}\text { EGFR }\left(K_{D}=21\right) \\
\operatorname{HER} 3\left(K_{D}=19\right)[445]\end{array}$} & \multirow{2}{*}{ MDA-MB-468 } & \multirow{2}{*}{$\begin{array}{c}\text { Down: p-AKT (T308), p-EGFR } \\
\text { (Y1068), p-ERK (T202/Y204), p-HER3 } \\
\text { (Y1289) in FaDu, the hypopharyngeal } \\
\text { carcinoma cell line }\end{array}$} & Cell & $\begin{array}{l}\text { - Reduced cell proliferation and mammosphere } \\
\text { formation }\end{array}$ & \multirow[t]{2}{*}{445} \\
\hline & & & & & & Xeno & $\begin{array}{l}\text { - Reduced tumor growth with concordant reduction } \\
\text { of CSCs }\end{array}$ & \\
\hline 2020 & $\begin{array}{r}\text { Pan-HE } \\
\text { (combination } \\
\text { each targe } \\
\text { epitopes of } \mathrm{E}\end{array}$ & $\begin{array}{l}\text { body mixture } \\
\text { sets of } 2 \text { antibodies } \\
\text { on-overlapping } \\
\text { HER2, and HER3) }\end{array}$ & - & 15 PDXs & $\begin{array}{c}\text { Down: p-AKT (T308), EGFR, p-EGFR } \\
\text { (Y1068), p-ERK1/2, p-FAK (Y397), } \\
\text { HER3, p-HER3 (Y1289) }\end{array}$ & PDX & - Reduced tumor growth & [446] \\
\hline
\end{tabular}


Table 3. Cont.

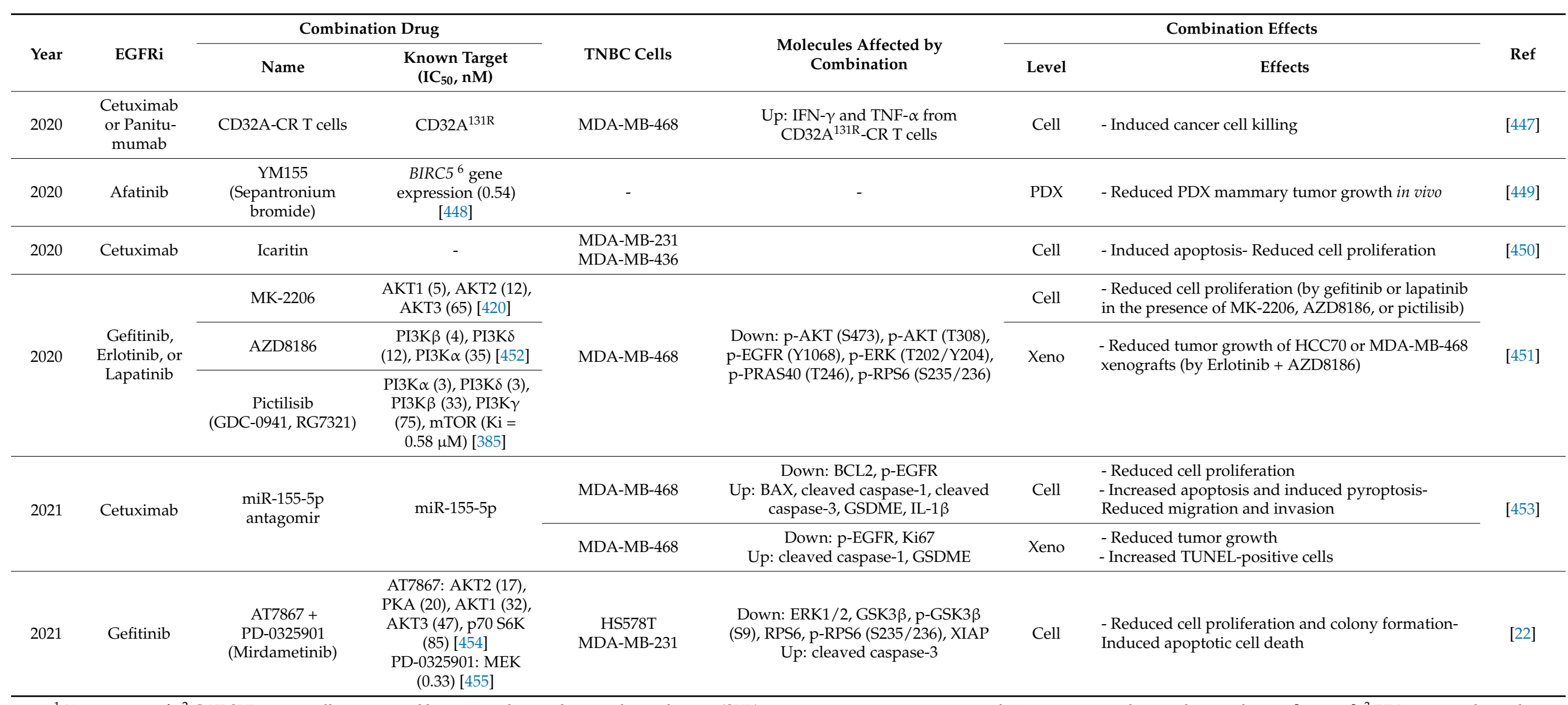

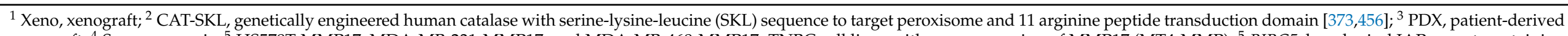

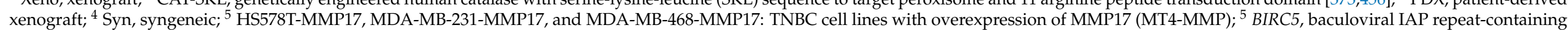
protein 5 ; the gene encoding survivin. 
Table 4. FDA-approved PKIs that have been experimentally tested with EGFRis in TNBC.

\begin{tabular}{|c|c|c|c|c|c|}
\hline Drug & Primary Target & $\begin{array}{l}\text { Initial Year } \\
\text { Approval }\end{array}$ & Company & Approved Indications ${ }^{1}$ & EGFRi Tested \\
\hline Crizotinib & ALK, ROS1, MET & 2011 & Pfizer & $\begin{array}{l}\text { ALK -positive advanced } \\
\text { NSCLC } \\
\cdot \quad \text { ROS1-positive NSCLC } \\
\cdot \quad \text { ALK-positive anaplastic } \\
\text { large cell lymphoma }\end{array}$ & Erlotinib [400] \\
\hline Dasatinib & BCR-ABL, SRC & 2006 & $\begin{array}{l}\text { Bristol-Myers } \\
\text { Squibb }\end{array}$ & $\begin{array}{l}\cdot \text { CML } \\
\cdot \quad \text { Philadelphia } \\
\text { chromosome (Ph)-positive } \\
\text { ALL } \\
\cdot \quad \text { Ph-positive CML }\end{array}$ & $\begin{array}{l}\text { Cetuximab } \\
{[376,440]}\end{array}$ \\
\hline Everolimus & mTOR & 2009 & Novartis & $\begin{array}{l}\text { HER2-negative breast } \\
\text { cancer, pancreatic } \\
\text { neuroendocrine tumors, } \\
\text { renal cell carcinoma, } \\
\text { angiomyolipoma, } \\
\text { subependymal giant cell } \\
\text { astrocytoma }\end{array}$ & $\begin{array}{l}\text { AEE788 [430] } \\
\text { Gefitinib [442] }\end{array}$ \\
\hline Imatinib & BCR-ABL & 2002 & Novartis & $\begin{array}{l}\text { Rare gastrointestinal } \\
\text { cancer } \\
\cdot \quad \text { ALL }\end{array}$ & Lapatinib [397] \\
\hline Palbociclib & $\mathrm{CDK} 4 / 6$ & 2015 & Park Davis & $\begin{array}{l}\text { ER- and HER2-positive } \\
\text { breast cancer }\end{array}$ & Erlotinib [428] \\
\hline Sirolimus & mTOR & 1999 & Wyeth & $\begin{array}{l}\text { Kidney transplant, } \\
\text { lymphangioleiomyomatosis }\end{array}$ & Lapatinib [368] \\
\hline Selumetinib & MEK1/2 & 2020 & Astra Zeneca & $\begin{array}{l}\text { Neurofibromatosis type } 1 \\
\text { plexiform neurofibromas }\end{array}$ & Gefitinib [393] \\
\hline Temsirolimus & mTOR & 2007 & Wyeth & Advanced kidney cancer & Gefitinib [387] \\
\hline Tepotinib & MET & 2021 & EMD Serono & $\begin{array}{l}\text { Metastatic NSCLC with } \\
M E T^{\text {ex14 }} \text { skipping } \\
\text { alterations }\end{array}$ & Gefitinib $[153,424]$ \\
\hline Trametinib & MEK1/2 & 2013 & GlaxoSmithKline & Melanoma & $\begin{array}{c}\text { Afatinib (with } \\
\text { Dasatinib) [444] }\end{array}$ \\
\hline
\end{tabular}

${ }^{1}$ https://www.drugs.com/, accessed on 15 April 2021.

\subsubsection{Combination with Other EGFRis}

Interestingly, among 60 preclinical studies, no study has reported on the combination of different generation EGFR TKIs in TNBC (Table 3). As mentioned earlier (see Section 3.1. EGFR Mutations or Amplification), this may reflect the fact that TNBC has been reported to possess rare EGFR mutations.

Another interesting strategy is the combination of small-molecule EGFRis and antiEGFR antibodies. Since EGFRis target the intracellular kinase domain and anti-EGFR antibodies bind to the ectodomain, the mode of action would be complementary $[364,403]$ This hypothesis has been proven in several studies. A combination of gefitinib with cetuximab was evaluated across various cancer cell lines, including breast, colon, prostate, and vulvar squamous carcinoma cells [364]. A TNBC cell line MDA-MB-468 was demonstrated to be a susceptible cell line in response to the gefitinib and cetuximab combination, whereas MDA-MB-435S did not respond to this combination [364]. MDA-MB-435S has been shown to express no detectable EGFR [459]. A combination of cetuximab or panitumumab with erlotinib or gefitinib has shown an anticancer effect in TNBC cell lines that harbor EGFR 
amplification (MDA-MB-468) and wild-type KRAS and PTEN (SUM1315MO2) [403]. This combination induced $\mathrm{G}_{1}$ cell cycle arrest and subsequent apoptosis with concomitant inhibition of the RAS/RAF/MEK/ERK pathway. Susceptible TNBC cells were sensitive to anti-EGFR antibody alone, while TNBC cells containing mutations in KRAS (MDA-MB-231) or PTEN (HCC1937) were resistant to anti-EGFR antibody treatment. Consistent with these results, anti-EGFR antibody alone could not inhibit the RAS/RAF/MEK/ERK pathway in resistant cells [403].

\subsubsection{Combination with MET Inhibitors (METis)}

As mentioned, MET activation confers resistance to EGFRis in TNBC cells $[152,153]$ (see Section 3.2. Activation of Bypass Signaling Pathways). An earlier study demonstrated that the addition of the METi tepotinib reduces resistance of MDA-MB-468 cells to gefitinib $[153,424]$. Interestingly, the gefitinib and tepotinib treatment together reduced cell growth of MDA-MB- 468 cells but not that of MDA-MB-231 cells, whereas the same combination reduced colony formation of both cell lines. Furthermore, the addition of cetuximab to tepotinib had no effect on MDA-MB-231 colony formation but had an effect on MDA-MB-468 colony formation. Mechanistically, the gefitinib and tepotinib treatment synergistically reduced levels of p-AKT (T308), p-ERK (T202/Y204), and p-ribosomal protein S6 (p-RPS6) (S235/236) in MDA-MB-468 cells, while no significant suppression was observed by single-agent treatment [153]. More interestingly, signal activation, including p-MET, p-EGFR, p-HER2, p-AKT, and p-ERK signaling, was provoked by EGF and HGF treatment of susceptible cells (MDA-MB-468) but not of non-susceptible cells (MDA-MB231 and T47D). Antibody microarray analysis identified that p-MET and p-HER3, which were induced by EGF and HGF co-treatment, were abolished by the gefitinib and tepotinib combination in MDA-MB-468 cells. In addition, knockdown of EGFR combined with the METi PHA-665752 or the erlotinib and PHA-665752 combination synergistically reduced the cell viability of TNBC cells in vitro [150].

The MSL subtype TNBC cell lines HS578T and MDA-MB-231 have been reported to be more resistant to gefitinib than BL subtype cell lines such as MDA-MB-468 and SUM149PT [15,378]. The addition of the METi SU11274 potentiated the cytotoxicity of gefitinib and reduced X-linked inhibitor of apoptosis (XIAP). Most interestingly, the gefitinib and SU11274 treatment markedly reduced the levels of both total RPS6 and p-RPS6 (S235/236). The reduction in the total RPS6 occurred as early as $2 \mathrm{~h}$ and was sustained over time to $16 \mathrm{~h}$ post-treatment, whereas the reduction in p-AKT (S473) returned to normal levels at $24 \mathrm{~h}$ post-treatment. Furthermore, knockdown or RPS6 alone reduced proliferation of HS578T and MDA-MB-231 TNBC cells [15]. The mechanism of RPS6 reduction achieved by this combination remains to be determined.

A combination of METis and EGFRis was further evaluated with the METis glexatinib (MGCD265) and crizotinib (PF-02341066) and the EGFRi erlotinib [400]. Glexatinib and crizotinib are multitarget, ATP-competitive inhibitors of DDR2, AXL, MERTK, PDGFR $\alpha$, VEGFR3 (FLT4), FLT3, MET, VEGFR2 (KDR), and PDGFR $\beta$ [399]; and of ROS1, ALK, LTK, FER, FES, MET, PTK2B (FAK2), TNK2, PTK2 (FAK), TRKA/B/C, and FRK (PTK4), respectively [401,402]. Interestingly, crizotinib also inhibits EGFR ${ }^{\text {L858R/T790M }}$ and EGFR $^{\mathrm{T} 790 \mathrm{M}}$ [402]. The addition of glexatinib or crizotinib to erlotinib synergistically reduced tumor growth in a patient-derived xenograft (PDX) model with consistent reduction in p-ERK1/2 and p-RPS6 in PDX tumor samples [400].

The MET and VEGFR inhibitor foretinib (XL880) [422] has been identified as a potentiator of the dual EGFR and HER2 inhibitor lapatinib in reducing the viability and migration of TNBC cells [423]. Furthermore, the invasiveness of TNBC cells was reduced by the lapatinib and foretinib combination, with robust reductions in the number of invadopodia formed and the ability of gelatin digestion. This combination could reduce p-AKT (S473) in BT549 and MDA-MB-231 cells but not p-ERK1/2 (T202/Y204) in MDA-MB-231 cells. Further study is needed to determine the molecular mechanism of this combination effect. 


\subsubsection{Combination with PI3K/AKT/mTORC1 Inhibitors}

The first report on the potentiation of EGFRis via vertical inhibition of the PI3K/AKT/ mTORC1 pathway demonstrated that PI-103 enhanced apoptotic cell death in the BL subtype TNBC cell lines MDA-MB-468 and SUM149PT [378]. No potentiation was observed in the MSL TNBC cell lines HS578T and MDA-MB-231. Synergistic reduction of anti-apoptotic proteins, including XIAP, BCL2, and MCL1, was detected in sensitive cells. Overexpression of these anti-apoptotic proteins is associated with drug resistance and a poor prognosis [253] (See Section 3.4.2. Overexpression of Anti-apoptotic Proteins). For example, MCL1 is regulated at multiple levels in cancer cells. Transcription factors such as STAT3 and MYC activate MCL1 transcription [460]. In addition, inhibitory phosphorylation by AKT alleviates the GSK3 $\beta$-mediated destabilization of MCL1. AKT further promotes the stability of MCL1 through its phosphorylation by mTORC1. A chemically modified LY294002 with the RGDS integrin-targeting peptide was also reported to potentiate gefitinib [461] in MDA-MB-468 TNBC cells [460]. The peptide leads to targeting of the RGDS integrins $\alpha v \beta 3$ and $\alpha 5 \beta 1$ and is released to liberate LY294002 by hydrolysis at physiological $\mathrm{pH}$ [462].

Dual blocking of EGFR and PI3K $\beta$ has been identified as an effective way to overcome the compensatory activation of the EGFR downstream signaling in a G protein-coupled receptor of thrombin (PAR1/F2R)-dependent manner [451]. A genome-wide shRNA screening approach has identified EGFR as an additional target that synergizes with PI3K inhibitors and AKT inhibitors in the PTEN-deficient TNBC cell line MDA-MB-468. Pharmacological inhibition of EGFR by gefitinib in combination with AZD8186, which is a PI3K inhibitor that specifically targets PI3K $\beta$, PI3K $\delta$, and PI3K $\alpha$ (Table 3) [452], synergistically reduces cell proliferation of PTEN-null TNBC cells but not that of PTEN wild-type TNBC cells [451]. This synergism was confirmed in vivo in orthotopic xenograft models of HCC70 or MDA-MB-468 cells by the erlotinib and AZD8186 combination. In addition, this combination also showed anticancer effects in an immunocompetent syngeneic mouse model. Among the components in the PI3K/AKT/mTORC1 pathway, the response of p-RPS6 (S235/236) represents a useful predictive marker for sensitivity to PI3K inhibition. A co-immunoprecipitation assay revealed that $P I 3 K \beta$ is a key component of the activated PI3K/AKT/mTORC1 pathway in PTEN-null TNBC cells. Targeted deletion screening by CRISPR-Cas9 further identified that targeting G protein $\beta$ and $\gamma$ subunits sensitized TNBC cells to EGFRis and PI3Kis. Inhibiting G protein $\beta$ and $\gamma$ subunits blocked the PAR1dependent activation of AKT. Vorapaxar, an inhibitor of PAR1, consistently synergizes the effect of pictilisib or lapatinib to reduce colony formation of MDA-MB-468 cells [451].

Rapamycin (silorimus) is a specific mTOR inhibitor that directly inhibits mTORC1 $[463,464]$. Rapamycin, in combination with lapatinib, has been reported to reduce the increase in xenograft tumors of MDA-MB-231 and MDA-MB-468 cells [368]. The lapatinib and rapamycin combination preferentially induces apoptosis in MDA-MB-468 cells compared to MDA-MB-231 cells both in vitro and in vivo. Similar to previous reports $[465,466]$, the level of p-eIF4E was associated with apoptotic resistance of MDA-MB-231 cells [368].

Analogs of rapamycin (rapalogs), including temsirolimus, everolimus, and deforolimus, have been developed as mTORC1 inhibitors [467]. A separate study reported that the blocking of mTOR by temsirolimus in the presence of gefitinib reduces cell growth, viability, colony-forming ability, and cap-dependent translation, with concomitant decreases in p-eIF4B in the TNBC cell lines BT20, MDA-MB-231, and MDA-MB-468 [387]. Similar to RPS6 [468], eIF4B is a converging point of the RAS/RAF/MEK/ERK and $\mathrm{PI3K} / \mathrm{AKT} / \mathrm{mTORC1}$ pathways [469]. Interestingly, p-p90RSK (S380) rather than p70S6K (T389) was found to be a mediator of this combination effect in these TNBC cells.

When combined with the EGFRi, AEE788 rapalogs induce synergistic reduction of TNBC cell proliferation [430]. An antiproliferation screening of 378 small-molecule PKIs in combination with rapamycin was performed against 19 TNBC cell lines. Multiple EGFRis, such as AEE788, afatinib, AC480, AZD8931, AZD9291, AST-1306, and gefitinib, have been shown to induce antiproliferative effects synergistically or additively in combination with rapamycin in the TNBC cell line SUM149PT. AEE788 is a dual inhibitor 
of EGFR and HER2, with $\mathrm{IC}_{50}$ values of 2 and $6 \mathrm{nM}$, respectively [470]. AEE788 also inhibits ABL1 $\left(\mathrm{IC}_{50}=52 \mathrm{nM}\right)$, FLT1 $\left(\mathrm{IC}_{50}=59 \mathrm{nM}\right)$, FMS $\left(\mathrm{IC}_{50}=60 \mathrm{nM}\right), \mathrm{SRC}\left(\mathrm{IC}_{50}=61 \mathrm{nM}\right)$, $\mathrm{KDR}\left(\mathrm{IC}_{50}=77 \mathrm{nM}\right)$, and HER4 $\left(\mathrm{IC}_{50}=160 \mathrm{nM}\right)$. The AEE788 and rapamycin combination induced apoptosis in SUM149PT as well as HCC1143 cells [430]. In contrast, this combination did not have antiproliferative effects or induce apoptosis in normal mammary cells (MCF10A cells) and renal cells (RPTEC cells). This combination abolished p-mTOR (S2448), while no significant inhibition of p-mTOR was observed after single-agent treatment. PAKT (S473) and p-ERK1 (T202/Y204) were inhibited by AEE788, and this inhibition was sustained by the combination. In addition, siRNA-based screening further revealed that RPS6KA3, RPS6KA6, RPS6KB1, and RPS6KL1 appear to be synthetic lethal targets for the AEE788 and rapalog combination treatment. An interesting point was that treatment with rapalogs induced the expression of both protein and mRNA of cyclin D1, while the addition of AEE788 abolished the rapalog-induced cyclin D1 expression in TNBC cells. Furthermore, the AEE788 and rapalog combination downregulated the expression of cyclin B1 and CDK4.

The susceptibility of TNBC cells to the EGFRi and mTORi combination has been shown to be correlated with the PIK3CA mutation status. The gefitinib and everolimus combination was effective in PIK3CA-mutatnt CAL-51 cells but not in wild-type HCC1937 cells [442]. CAL-51 cells contain a heterozygous E542K mutation in the PIK3CA gene with the PTEN-null background [442,471]. These results are consistent with the fact that the PIK3CA mutation amplifies AKT activation by PTEN loss in TNBC cells [19]. The combination reduced the levels of p-4E-BP1 (T37/46) and p-mTOR (S2448) in susceptible cells. No significant effects were observed on p-AKT (S473), whereas downregulation of p-ERK (T202/Y204) was observed. The combination further reduced the levels of cyclin B1 and cyclin E1 protein and mRNA, with a concordant increase in $\mathrm{G}_{1}$ cell cycle arrest and apoptosis. Gene expression profiling analysis identified upregulation of genes involved in DNA damage repair and cell cycle progression, such as checkpoint kinase 1 (CHEK1), CHEK2, cyclin A1 (CCNA1), CCNB1, and CCNE1.

Another study further supported the notion that the mTORC1 serves as a promising target for sensitizing TNBC cells to EGFRis. Combined treatment with gefitinib and MK2206, a selective AKT inhibitor [420], synergistically reduced cell viability and colony formation of the TNBC cell lines HS578T and MDA-MB-213 [421]. More importantly, knockdown of the regulatory-associated protein of mTOR (RPTOR), but not rapamycininsensitive companion of mTOR (RICTOR), confirmed the effects of MK-2206 in terms of the reductions in cell viability and total RPS6 levels in TNBC cells in the presence of gefitinib. Since RPTOR is the mTORC1-specific component [464], these results support the idea that the selective targeting of mTORC1 is a potential strategy to overcome EGFRi resistance.

Similar to small-molecule EGFR TKIs, a combinatorial benefit of vertical inhibition has also been reported with an anti-EGFR antibody in combination with either a PI3Ki or an AKTi. Duligotuzumab is a dual-acting human IgG1 monoclonal antibody against EGFR and HER3 [472,473]. Combined treatment with duligotuzumab and ipatasertib (a pan-AKTi) [384] or pictilisib (a pan-PI3Ki) [385] showed anticancer effects in TNBC cells both in vitro and in vivo, with prolonged inhibition of EGFR or HER3 signaling [159]. As expected, the duligotuzumab and pictilisib combination was more effective than the cetuximab and pictilisib combination in the regression of tumor growth of HCC70 xenografts, since inhibition of EGFR, AKT, or PI3K induced the abundance of HER3 [474-476], which was inhibited by duligotuzumab but not by cetuximab.

\subsubsection{Combination with RAS/RAF/MEK/ERK Inhibitors}

Treatment with the MEK inhibitor selumetinib was shown to induce p-AKT (S473) in a panel of TNBC cells [393]. Combined treatment with selumetinib and gefitinib synergistically reduced the viability of TNBC cells and induced $\mathrm{G}_{0} / \mathrm{G}_{1}$ cell cycle arrest and apoptosis in selumetinib-sensitive SUM149PT cells. The gefitinib and selumetinib combination showed nearly complete inhibition of p-ERK1/2 (T202/Y204) and a further reduction 
in the selumetinib-mediated p-AKT (S473) level. In contrast, this combination did not induce $\mathrm{G}_{0} / \mathrm{G}_{1}$ cell cycle arrest and did not reach the $\mathrm{IC}_{50}$ value in the selumetinib-resistant TNBC cells MDA-MB-468 and SUM159PT. However, the molecular mechanism of this difference remains elusive.

\subsubsection{Combination with IKK/NF-KB Inhibitors}

The therapeutic potential for targeting NF- $\mathrm{kB}$ in combination with EGFRis has been demonstrated by bortezomib [380]. Bortezomib (Velcade ${ }^{\circledR}$ ) is the first FDA-approved proteasome inhibitor for the treatment of multiple myeloma [477]. One mode of action of bortezomib is downregulation of NF- $\mathrm{kB}$ activity through blocking proteasomal degradation of IкB $[379,478]$. As mentioned, activation of the NF- $\kappa B$ pathway has been described in ER-negative breast cancer cells [209,479]; however, a phase 2 clinical trial resulted in limited benefits [480]. Lapatinib, but not gefitinib or erlotinib, has been demonstrated to be able to induce activation of NF- $\mathrm{kB}$ through SRC-dependent $\mathrm{p} 65$ and I $\mathrm{KB} \alpha$ phosphorylation [380]. Studies have shown that lapatinib upregulates SRC activity in an EGFR/HER2-independent manner. This lapatinib-induced NF- $\mathrm{KB}$ activation leads to the synergistic anticancer activity of co-treatment with lapatinib and bortezomib both in vitro and in vivo in two TNBC cells, HS578T and MDA-MB-231 [380]. Consistent with this finding, our group has identified that the combination of an IKK inhibitor and the EGFRi gefitinib has an anticancer effect in TNBC cells (You et al., manuscript in preparation).

\subsubsection{Combination with JNK Inhibitors}

JNK-IN-8 is the first irreversible JNK inhibitor for JNK1, JNK2, and JNK3, with $\mathrm{IC}_{50}$ values of 4.7, 18.7, and $1 \mathrm{nM}$, respectively [408]. It covalently binds to C116 of JNK1 and JNK2, inhibiting phosphorylation of its target, c-Jun, at S63. Recently, the combination of lapatinib and JNK-IN-8 has been demonstrated to induce apoptosis of the TNBC cell lines, HCC1569, MDA-MB-231, and MDA-MB-435, in vitro and to retard MDA-MB-231 xenograft tumor growth in vivo [231]. The lapatinib and JNK-IN-8 combination reduced the transcriptional activities of NF- $\mathrm{KB}$, activating protein 1 (AP-1), and NRF2 in TNBC cells, leading to an increase in the ROS level. As mentioned, NRF2 is a master regulator of a battery of genes involved in antioxidant responses, drug resistance, and detoxification [155]. Of importance, NRF2-KD reduces the expression of CSC markers, such as ALDH1A1 and $A L D H 3 A 1$, in pancreatic cancer cells [481].

\subsubsection{Combination with CDK Inhibitors}

In humans, twenty-one cyclin-dependent kinases (CDKs) have been identified based on sequence similarity [482]. The CDK family is composed of two major subgroups of protein kinases: (1) those that regulate cell cycle progression (CDK1, CDK2, CDK4, and CDK6); (2) those that play roles in transcriptional processes (CDK7, CDK8, CDK9, CDK12, and CDK13) [483-485]. The functions of the remaining CDKs are still under investigation [482,484]. Dysregulation of CDKs has been associated with tumorigenesis or cancer progression, and they have been well established as anticancer targets $[484,485]$.

Cell cycle progression is regulated by interplay between CDKs, cyclins, retinoblastoma (RB), CDK inhibitors, and transcription factor E2Fs [486,487]. Matrix metalloproteinase-17 (MMP17; also known as membrane-type-4 matrix metalloproteinase, MT4-MMP) has been identified as a copartner and is co-expressed with EGFR in approximately $80 \%$ of clinical TNBC samples. Its expression sensitizes TNBC cells to erlotinib $[488,489]$. In a subsequent study, dual targeting of EGFR and CDK4/ 6 by erlotinib and palbociclib, respectively, is additively effective in reducing tumor growth in TNBC xenografts and PDX cells expressing MMP17, EGFR, and RB, whereas PDX-TNBC cells without RB expression were resistant to this combination [428]. Interestingly, the expression of these markers has been found in approximately $50 \%$ of TNBC samples, which is useful to predict the sensitivity of TNBC to EGFR and CDK4/6 dual inhibition. 
CDK9 is a catalytic subunit of a multiprotein complex, positive transcription elongation factor b (P-TEFb) [490], and is activated by CDK7 [491]. Both CDK9 and CDK7 positively activate gene transcription through sequential phosphorylation of the C-terminal domain of RNA polymerase 2 at S5/7 and S2, respectively [492-494].

CDK7 has been noted as a novel anticancer target, and its mRNA expression is linked with a poor prognosis in TNBC [495]. A covalent inhibitor of CDK7, THZ1 [437], has been reported to effectively repress the proliferation of breast cancer cells [496,497]. Very recently, the combination of THZ1 and erlotinib was identified to have synergistic or additive anticancer effects on various subtypes of breast cancer in vitro, including TNBC [438]. Although THZ1 represses a variety of genes such as CDKN1B, MYC, EGFR, $F O X C 1, P L K 2$, and CTED2, the mechanism of action of the combination of erlotinib and THZ1 remains to be elucidated.

A dual PKI of cell division cycle 7-related protein kinase (CDC7) and CDK9, PHA767491, has been identified as a sensitizer of EGFRi in TNBC cells through a high-throughput screening of 273 PKIs in combination with lapatinib [99]. The synergism of the lapatinib and PHA-767491 combination in antiproliferative activity has been confirmed across 17 TNBC cell lines. This combination reduced CDC7 levels and phosphorylation of its downstream target, minichromosome maintenance protein 2 homology (MCM2), at S40/41. Since MCM2 is a crucial component of DNA helicase [498], the reduction of p-MCM2 suggests inhibition of DNA replication initiation by this combination. In addition, this combination reduced the CDK9-mediated phosphorylation of the DNA-directed RNA polymerase 2 subunit A (POLR2A) and the level of total POLR2A. This synergism is EGFR-specific, since EGFR TKIs, such as erlotinib and gefitinib, also resulted in synergism with PHA-767491. The lapatinib and PHA-767491 combination further repressed the levels of CDK4, cyclin D1, and $p-R B(S 780)$, with a concordant reduction in the $G_{0} / G_{1}$ phase and induction of $G_{2} / M$ cell cycle arrest. EGFRi and CDC7/CDK9i-induced $\mathrm{G}_{2} / \mathrm{M}$ cell cycle arrest is consistent with abnormal DNA replication origin activation checkpoint in cells, with loss-of-function $p 53$ mutations that are commonly found in TNBC cells [99,120,192,240,499]. This combination also induced apoptosis in TNBC cell lines. In clinical ER-negative breast cancer samples, high expression levels of CDC7 and POLR2A were associated with a poor prognosis [99].

\subsubsection{Combination with SFK Inhibitors}

Human SRC family kinases (SFKs) are a group of non-receptor tyrosine kinases, including B lymphocyte kinase (BLK), Gardner-Rasheed feline sarcoma viral oncogene homolog (FGR), FYN-related kinase (FRK), proto-oncogene c-Fyn (FYN), hematopoietic cell kinase (HCK), lymphocyte-specific protein tyrosine kinase (LCK), Lck/Yes-related novel protein tyrosine kinase (LYN), v-src avian sarcoma (Schmidt-Ruppin A-2) viral oncogene homolog (SRC), and v-yes-1 Yamaguchi sarcoma viral oncogene homolog 1 (YES1) $[500,501]$. SFKs play crucial roles in regulating signal transduction provoked by cell surface receptors.

As mentioned earlier, anti-EGFR antibodies may exert an agonistic action on EGFR, leading to resistance of TNBC cells to anti-EGFR antibody therapeutics (see Section 3.2. Activation of Bypass Signaling Pathways). Recently, the combination of cetuximab and the SFK inhibitor PP2 was found to overcome this resistance in the TNBC cell lines MDAMB-231 and MDA-MB-468 [142]. Cetuximab alone induced phosphorylation of PI3K and SRC. In addition, cetuximab activated RTKs, including IGF1R and VEGFR2. The combination of cetuximab and PP2 abolished cetuximab-induced p-IGF1R and p-VEGFR2, with a concordant reduction in cell proliferation.

Dasatinib is an orally available TKI of ABL1, SRC family kinases, KIT, and PDGFR $\alpha / \beta$, and has been approved for the treatment of chronic myeloid leukemia (CML) and Philadelphiachromosome-positive acute lymphoblastic leukemia (Ph+ ALL) (Table 4) [502,503]. Treatment with dasatinib alone reduced p-EGFR (Y845) and p-SRC (Y416), with a concordant reduction in nuclear EGFR and SRC and induction of radio-labeled cetuximab binding to the cell surface in TNBC cell lines such as MDA-MB-231 and MDA-MB-468 cells [440]. 
In an MDA-MB-468 xenograft model, the cetuximab and dasatinib combination further reduced the tumor volume compared with dasatinib alone, while no significant change was observed in an MDA-MB-231 xenograft model with this combination treatment [440]. Although the noted difference in these cell lines was a KRAS mutation (MDA-MB-468, wildtype vs. MDA-MB-231, mutated), further study is needed to determine the contributions of KRAS mutations to the differential effects of this combination.

A benefit of the combination of dasatinib with the EGFRi afatinib has also been reported [441]. The afatinib and dasatinib combination was synergistic or additive in 13 out of 14 TNBC cell lines. Interestingly, low levels of BCL2 and mTOR were associated with the synergism of this combination. P-EGFR (Y1068) and p-SRC (Y527) levels were commonly suppressed by the afatinib and dasatinib combination, with a concordant increase in $\mathrm{G}_{1}$ cell cycle arrest and without significant induction of apoptosis. The anticancer effect of this combination was confirmed in a TNBC xenograft model with decreases in CDC42 and p-EGFR (Y1068) in the xenograft tumor [441].

\subsubsection{Combination with ABL1 Inhibitors}

Additional blocking of Abelson murine leukemia viral oncogene homolog 1 (ABL1) by imatinib overcomes EGFRi resistance in TNBC cells. Co-treatment of the TNBC cell lines HCC1806, MDA-MB-231, MDA-MB-468, and SUM159PT with imatinib and lapatinib resulted in a reduction in nuclear $\beta$-catenin accumulation, leading to the suppression of the tumor-promoting transcription factor MYC [397]. More interestingly, the expression of the long non-coding RNA (lncRNA) HOX antisense intergenic RNA (HOTAIR) was suppressed by the imatinib and lapatinib combination treatment. Nuclear $\beta$-catenin has been shown to bind the promoter region of HOTAIR according to a chromatin immunoprecipitation (ChIP) assay. HOTAIR is known to promote tumor progression and is associated with a poor prognosis [504-506]. The combination also reduced the tumor size of the MDA-MB231 xenograft [397].

\subsubsection{Combination with Focal Adhesion Kinase (FAK) Inhibitors}

FAK1 and PYK2 (or FAK2) are non-receptor tyrosine kinases controlling cell shape, adhesion, and motility [410]. High expression levels of FAK1 and activated PYK2 are associated with TNBC [507-509]. High expression levels of EGFR and PYK2 have been further associated with a poor prognosis among patients with TNBC [161]. In addition, IL-25 has been demonstrated to activate PYK2 and induce EGFRi resistance in TNBC cells [347] (See Section 3.4.10. Induction of Autophagy).

The combination of the FAK1 inhibitor (FAK1i) PF573228 [409] or the PYK2 inhibitor (PYK2i) PF431396 [411] with gefitinib or erlotinib synergistically reduced TNBC cell proliferation in vitro and tumor growth in vivo [161]. This synergism was also confirmed by shRNA-based knockdown of FAK1 or PYT2. The FAK1i/PYK2i and EGFRi combination inhibited the activation of the AKT/S6K, STAT3, and ERK1/2 pathways (Table 3). Furthermore, PYK2 blocking reduced EGFRi-induced HER3 upregulation through Nmyc downstream-regulated gene 1 (NDRG1)-mediated proteasomal degradation. Mechanistically, PYK2 blocking upregulated NDRG1 expression, leading to enhanced interactions of HER3 with its E3 ubiquitin ligase, NEDD4. NDRG1 is a metastasis suppressor in human cancers such as breast, colon, and prostate cancers [510]. Of importance, NRDG1 interacts with multiple signaling pathways, including the PI3K/AKT/mTORC1, RAS/RAF/MEK/ERK, and IKK/NF-KB pathways. However, the mechanism of EGFRiinduced NRDG1 expression remains to be determined. Co-targeting of PYK2 by PF431396 has also been reported to suppress the gefitinib-induced rebound of p-ERK1/2 (T202/Y204) and p-STAT3 (Y705) in MDA-MB-468 cells [424].

4.1.11. Combination with Rho-Associated, Coiled-Coil-Containing Protein Kinase (ROCK) Inhibitors

Two in vivo and in vitro knockdown screenings have identified ROCK1 as a therapeutic target for TNBC [405]. Co-treatment with gefitinib and the ROCK inhibitor (ROCKi) 
GSK269962A reduced colony formation of a panel of TNBC cell lines, such as BT549, CAL-20, CAL-51, HCC38, HCC1806, HS578T, LM2, and MDA-MB-231 cells, compared with single-drug treatments. The combination inhibited the $S$ phase and increased $G_{2}$ cell cycle arrest. Cell cycle progression proteins, including cyclin A, CDK2, and p27, were consistently reduced, and the level of p-RB (S807/811) was also reduced in MDA-MB-231 cells treated with the combination, whereas little or no change in the levels of these proteins were observed in MDA-MB-231 cells treated with a single agent. In an orthotopic xenograft model with HCC1806 cells, tumor growth was reduced by the gefitinib and GSK269962A combination compared with the control or single agent treatment. Interestingly, the effect of the ROCKi alone in vivo was superior to that of gefitinib and not statistically different from that of the combination [405]. A subsequent study provided a more in-depth understanding of the mechanism of action of the gefitinib and GSK269962A combination [339]. Proteomic profiling of TNBC cell lines treated with the combination was compared to that of cells treated with a single drug alone; gefitinib was found to induce autophagy in TNBC cells, while the addition of the ROCKi blocked EGFRi-induced autophagy, leading to an anticancer effect in TNBC cells. In addition, the fact that another EGFRi afatinib [94,95] and ROCKi fasudil [511] resulted in similar anti-colony formation effects in TNBC cells suggests that the effect is a target-specific but not compound-specific [405]. Notably, fasudil has been approved in Japan and China, but not by the US FDA or the European Medicines Agency (EMA) [512].

\subsubsection{Triple Combination of PKIs}

Triple combinations have been applied to suppress rewired signaling pathways by single or dual inhibition [22]. In cancer cells, blocking two pathways with a combination may induce rewiring of a signaling pathway(s) to circumvent the inhibition of survival signals [102,103]. Additional blocking of these rewired signaling pathways might provide another opportunity. For example, dasatinib attenuates SRC signaling induced by the poly(ADP-ribose)polymerase inhibitor (PARPi) veliparib and the DNA damaging agent carboplatin in TNBC [513]. Dasatinib is an ATP-competitive kinase inhibitor of ABL1, SRC, KIT, and CSK $[374,375,502]$. SRC is a member of the SFKs, and its overexpression has been associated with aggressiveness of tumors, including TNBC [514-517]. SFKs are key components in cell signaling regulation (see Section 4.1.8. Combination with SFK Inhibitors).

An earlier study demonstrated that a triple combination of cetuximab, dasatinib, and cisplatin induced apoptosis synergistically in various TNBC cell lines [376]. In addition, the triple combination attenuated the cell growth and migration of TNBC cells. Single (cetuximab or cisplatin) or dual (cetuximab + cisplatin) treatment induced p-EGFR, pAKT, and p-ERK in a TNBC cell line-dependent manner, while the addition of dasatinib attenuated this activations [376]. Dasatinib may further contribute to the inhibition of EGFR through blocking of SRC-induced EGFR phosphorylation [376] and direct EGFR binding [518], and by inducing its lysomomal degradation [519]. Interestingly, dasatinib has been shown to reduce the expression of $A L D H 1 A 1$, leading to potentiation of gemcitabine in a gemcitabine-resistant pancreatic cancer cell line [520]. The ALDH family members consist of 19 isoforms, which have been proposed as hallmarks of drug resistance in CSCs [521]. The expression of $A L D H 1 A 1$ and $A L D H 3 A 1$ has also been linked to NRF2 in pancreatic cancer cells. NRF2-KD abolished the expression of ALDH1A1 and ALDH3A1, leading to sensitization of pancreatic cancer cells to 5-fluorouracil (5-FU) [480].

A combination screening of 33 FDA-approved PKIs identified a triple combination of afatinib, dasatinib, and trametinib as an effective strategy for TNBC treatment [444]. The drug combination discovery approach was designed to use a single-mixture solution of 33 PKIs at the start and then to use dropout solutions in the TNBC cell line HCC1143. The dropout solutions were made by exclusion of a group of kinase inhibitors that were classified according to their primary targets from the 33X mixture solution. The triple combination displayed antiproliferative activity in two TNBC cell lines, HCC1143 and 
MDA-MB-468. Although the mechanism of action remains to be investigated, the results suggest that simultaneous inhibition of the PI3K/AKT and MEK/ERK pathways is a promising approach to potentiate EGFRis in TNBC [444].

Very recently, the combination of gefitinib with the AKT inhibitor, AT7867, has been shown to induce activation of the MEK/ERK pathway in TNBC cell lines [22]. The addition of the MEK inhibitor PD-0325901 to the gefitinib and AT7867 combination resulted in the synergistic reduction of cell proliferation and colony formation of HS578T and MDA-MB231 cells. Apoptotic cell death, evident with the increased subG 1 phase, was only observed with the triple combination, with a concordant reduction in XIAP and increase in cleaved caspase-3. Interestingly, a reduction in the total RPS6 was observed in TNBC cells treated with the triple combination [22]. The importance of the total RPS6 in TNBC cell proliferation was previously demonstrated by RPS6-KD [15] (see Section 4.1.2. Combination with MET Inhibitors (METis)); thus, it is important to investigate the mechanisms of action of both intrinsic and acquired resistance to enrich our strategies to fight cancers.

Cumulative evidence supports the potential for enhancing EGFRi-mediated anticancer efficacy in TNBC by using combination strategies targeting EGFR and related signaling pathways in TNBC cells (Figure 2). As mentioned earlier, crosstalk and rewiring of signal transduction pathways contribute to either intrinsic or acquired EGFRi resistance in TNBC. Continuous efforts will provide a comprehensive understanding of the molecular mechanisms of EGFRi resistance and rationale for circumventing the limited efficacy of EGFRi in TNBC. The availability of small-molecule inhibitors for enzymes other than protein kinases will also facilitate future studies (see the following sections).

\subsection{Combination with Other Targeted Therapeutics}

\subsubsection{Combination with PARP Inhibitors (PARPis)}

PARP1 is the first therapeutic target of FDA-approved small-molecule inhibitors for TNBC treatment. Olaparib (Lynparza ${ }^{\circledR}$ ) and talazoparib (Talzenna ${ }^{\circledR}$ ) have been approved for germline $B R C A$-mutated metastatic breast cancer and germline BRCA-mutated, HER2negative, locally advanced or metastatic breast cancer, respectively; however, their efficacy is extending beyond tumors based on BRCA mutation status [522,523].

An earlier study demonstrated that the combination of lapatinib and the PARPi veliparib resulted in synergistic anti-clonogenic activity in the TNBC cell lines MDA-MB-231, MDA-MB-453, and MDA-MB-468 [372]. Of interest, these TNBC cell lines lack BRCA1 mutations [524]. This combination also induced intrinsic apoptosis, as evidenced by activation of caspase- 3 and caspase-9. This synthetic lethal interaction between lapatinib and veliparib is due to lapatinib-mediated homologous recombination repair deficiency, which mimics the BRCA1 mutation. Furthermore, lapatinib induced the translocation of BRCA1 and EGFR to the cytoplasm, preventing DNA repair in the nucleus, with a consistent increase in the DNA damage marker $\gamma$-H2AX. In addition, lapatinib disrupted the BRCA1-EGFR interaction. This combination attenuated MDA-MB-231 xenograft tumors [372]. Recently, negative regulation of EGFR expression by BRCA1 through transcriptional activation of miR-146a that targets the $3^{\prime}$-untranslated region (3'-UTR) of EGFR has been reported [525]. Mutations of $B R C A 1$ have been linked to the TNBC phenotype $[19,20]$.

Very recently, PARPi-resistant, BRCA1-mutated SUM149PT TNBC cell lines were developed, which demonstrate high levels of p-MET (Y1234/1235) and p-EGFR (Y1086) [436]. Knockdown of MET reversed PARPi resistance, while the triple combination of an EGFRi (gefitinib), a METi (crizotinib), and a PARPi (talazoparib) further reduced cell viability [436]. BEZ235, an inhibitor of PI3K, mTOR, ATM, ATR, and DNA-PK, has been identified as a potentiator of the PARPi olaparib in reducing colony formation of two BRCA1-mutated TNBC cell lines, MDA-MB-436 and SUM149PT, with reductions in olaparib-induced 53BP1 foci in SUM149PT cells [526]. Further studies on the interactions between EGFRis and PARPis will extend our understanding of TNBC biology and therapy. 


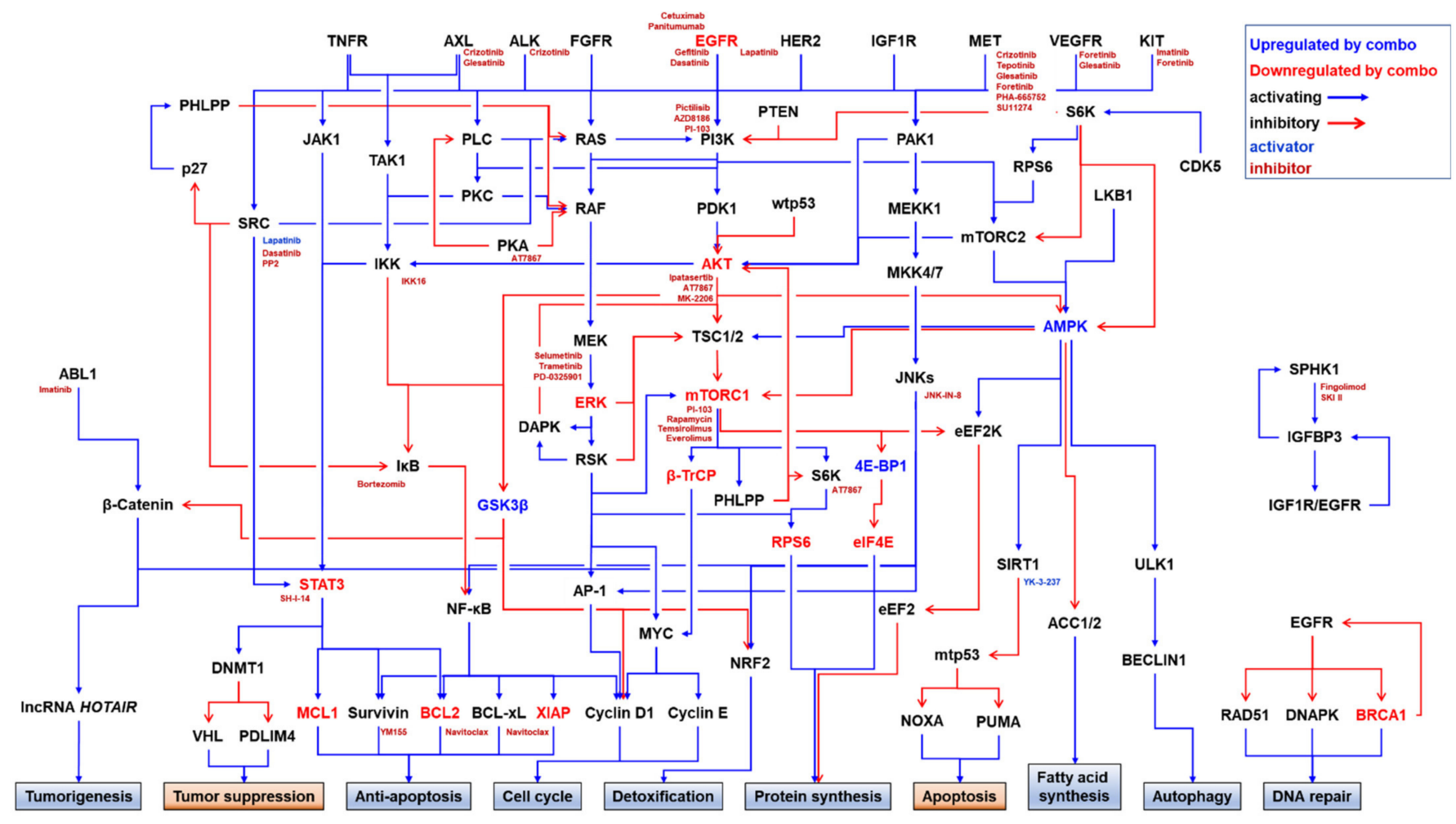

Figure 2. Schematic diagram of the putative EGFR and related signaling pathways in TNBC cells. Abbreviations: ABL1, Abelson murine leukemia viral oncogene homolog 1; ACC1/2, acetyl-CoA carboxylase 1/2; AKT, v-akt oncogene homolog; ALK, anaplastic lymphoma kinase; AMPK, $5^{\prime}$ adenosine monophosphate (AMP)-activated protein kinase; AP- 1 activator protein 1; AXL, Anexelekto receptor tyrosine kinase; BCL2, B-cell lymphoma 2; BCL-xL, B-cell lymphoma-extra-large; BRCA1, breast cancer type 1 susceptibility protein; $\beta$-TrCP, beta-transducin repeat-containing protein; CDK5, cyclin-dependent-like kinase 5; MYC, cellular myelocytomatosis; DAPK, death-associated protein kinase; DNAPK, DNA-dependent protein kinase; DNMT1, DNA (cytosine-5)-methyltransferase 1; eEF2, eukaryotic elongation factor 2; eEF2K, eukaryotic elongation factor 2 kinase; EGFR, epidermal growth factor receptor; eIF4E, eukaryotic translation initiation factor 4E; ERK, extracellularsignal-regulated kinase; FBW7, F-box and WD repeat domain-containing 7; FGFR, fibroblast growth factor receptor; GSK3 $\beta$, glycogen synthase kinase-3 beta; HER2, human epidermal growth factor receptor 2; IGF1R, insulin-like growth factor 1 receptor; IKB, nuclear factor of kappa light polypeptide gene enhance in B-cells inhibitor; IKK, IKB kinase; JAK1, Janus kinase 1; JNKs, c-Jun N-terminal kinases; KIT, v-kit Hardy-Zuckerman 4 feline sarcoma viral oncogene homology; LKB1, liver kinase B1; MCL1, myeloid-cell leukemia 1; MEK, MAPK/ERK kinase; MEKK1, mitogen-activated protein kinase kinase kinase 1; MET, mesenchymal-epithelial transition factor; MKK4/7, mitogen-activated protein kinase kinase 4; mTORC1/2, mammalian target of rapamycin complex 1/2; mtp53, mutant p53; NF- $\mathrm{B}$, nuclear factor of kappa light polypeptide gene enhanced in B-cells; PAK1, p21-activated kinase 1; PDLIM4, PDZ and LI domain 4; PHLPP, PH domain and leucine-rich repeat protein phosphatase; PI3K, phosphoinositide 3-kinase; PDK1, phosphoinositide-dependent kinase-1; PLC, phospholipase C; PKA, protein kinase A; PKC, protein kinase C; PTEN, phosphatase and tensin homolog; PUMA, p53-upregulated modulator of apoptosis; RAD51, RAD51 (S. cerevisiae) homolog; RAF, rapidly accelerated fibrosarcoma kinase; RAS, rat sarcoma; RPS6, ribosomal protein S6; RSK, ribosomal S6 kinase; S6K, S6 kinase; SIRT1, NAD-dependent deacetylase sirtuin-1; SRC, v-src avian sarcoma (Schmidt-Ruppin A2) viral oncogene homolog; STAT3, signal transducer and activator of transcription 3; TAK1, transforming growth factor beta-activated kinase 1; TNFR, tumor necrosis factor receptor; TSC1/2, tuberous sclerosis complex 1/2; ULK1, Unc-51-like autophagy-activating kinase 1; VEGFR, vascular endothelial growth factor receptor; VHL, Von Hippel-Lindau tumor suppressor; XIAP, X-linked inhibitor of apoptosis.

\subsubsection{Combination with Inhibitors of Anti-Apoptotic Proteins}

ABT-806 is a humanized, tumor-specific anti-EGFR IgG1 monoclonal antibody [527]. The antibody-drug conjugates (ADCs) ABT-414 (depatuxizumab mafodotin) [528] and ABBV-321 (serclutamab talirine) [529] are composed of the antibody ABT-806 conjugated to the cytotoxic monomethyl auristatin F (MMAF) and the affinity-maturated version of ABT- 
806 conjugated to an ultrapotent pyrrolobenzodiazepine dimer, respectively. Navitoclax (ABT-263) is a small-molecule inhibitor of BCL-xL, BCL2, and BCL2L2 with anticancer activity [433]. The combination of navitoclax with these ADCs has been identified as an effective strategy in multiple PDX models of TNBC [258]. After fourteen days of daily navitoclax treatment with ABT-414 once per week, five of seven PDX models showed reduced tumor growth and three of seven models showed tumor regression compared with the vehicle control. A further study involving daily navitoclax treatment for 5 days per week and MMAF-loaded ADC (ABT-414 or AB095-MMAF, a non-tumor-targeted ADC) treatment once per week including single-agent controls was performed. Interestingly, treatment with ABT-414 alone showed no effect, while treatment with navitoclax alone reduced the tumor volume by approximately $20 \%$. The ABT- 414 and navitoclax treatment further induced tumor regression by approximately $40 \%$ [258]. The combination effect was EGFR-mediated, since the non-tumor (tetanus toxoid)-targeted ADC had little or no synergistic effect with navitoclax. The combination effect of an alternative ADC, ABBV-321, with navitoclax further supports EGFR dependency.

A screening of 1363 drugs in vitro in ten patient-derived TNBC cell lines identified that the combination of afatinib and YM155, an inhibitor of baculoviral IAP repeat-containing protein 5 (BIRC5; gene encoding survivin) expression [448], reduced the growth of patientderived TNBC cells in vitro and the PDX mammary tumors in vivo [449]. However, the effect of the combination did not reach statistical significance compared with treatment with YM155 alone. This dominant effect of YM155 might be due to its ability to reduce EGFR expression in TNBC cells through an unknown mechanism. Clinically, high expression levels of EGFR and survivin are associated with metastasis-free survival [449].

As mentioned earlier (see Section 3.4.2. Overexpression of Anti-apoptotic Proteins), MCL1 has been reported to confer EGFRi resistance in an ELP complex-dependent manner [260]. In addition, co-treatment of the MCL1 inhibitor 563845 synergistically induced erlotinib sensitivity in TNBC cells. Further studies on the underlying mechanism of ELP-complex-dependent MCL1 expression will be of interest in understanding the newly identified oncogene addition in TNBC [260].

\subsubsection{Combination with Sphingosine Kinase (SPHK) Inhibitors}

SPHK1 has been linked to acquired drug resistance of various cancers to chemotherapeutics and receptor inhibitors [530-538]. A high level of SPHK1 has also been associated with poor OS and PFS among patients with TNBC [539]. One study demonstrated that the combination of the SPHK inhibitor SKI II with gefitinib reduced the growth of MDA-MB468 xenograft tumors [389]. SPHK1 converts sphingosine to sphingosine 1-phosphate (S1P), which provokes a variety of signaling pathways via binding to S1P receptors 1-5 (S1PR1-5). SPHK1 has been established as an oncogene, and its overexpression is associated with a poor clinical prognosis in most cancers [538,540]. More interestingly, a higher expression of SPHK1 has been found in TNBC than in ER-positive breast cancer and is associated with a poorer DFS [541]. In TNBC cells, pharmacological inhibition or siRNA-based knockdown of SPHK1 inhibits insulin-like growth factor-binding protein-3 (IGFBP3)-enhanced EGFR activation. Although the underlying molecular mechanism is still largely unknown, the combination of an SPHK1 inhibitor and gefitinib significantly reduced tumor growth in an MDA-MB-468 xenograft model [389].

Another SPHK1 pathway inhibitor, fingolimod (FTY720) [412,542], which is a clinically approved S1PR1 antagonist [543], has also been demonstrated as a potentiator of gefitinib in TNBC cells, especially in BL subtype cells, in vitro and in vivo [413]. The gefitinib and fingolimod combination is also antiproliferative in HCC70 (a BL2 subtype), MDA-MB-231 (an MSL subtype), and BT549 (an M subtype) cells. The shRNA-based knockdown of IGFBP3 abolished the synergism induced by the gefitinib and fingolimod combination. CD44 has been reported to be upregulated by both EGFR [544] and SPHK1 [545] and to be associated with a poor prognosis of TNBC [546] (see Section 3.4.8 for CD44 in EGFRi resistance). In addition to CD44 downregulation by gefitinib alone, further downregulation 
occurs with the addition of fingolimod to gefitinib in TNBC cell lines [413]. Furthermore, the gefitinib and fingolimod combination extended the survival of TNBC xenograft nude and syngeneic immune-competent mice. The DNA-damaging agent induced direct binding and nuclear co-localization of IGFBP3 to EGFR in TNBC cells [547]. In HCC1806 and MDA-MB-468 xenograft tumors, nuclear IGFBP3 was reduced in response to each drug and further reduced by the combination [413]. However, the mechanism of action of this combination remains to be investigated. Notably, a recent study demonstrated that the inhibition of SPHK1 activity either by siRNA or by PF-543, a sphingosine-competitive SPHK1 inhibitor [548], led to suppression of the Notch pathway, with reductions in migration and invasion of TNBC cells in vitro and in vivo [539]. In addition, blocking SPHK1 activity also enhanced the sensitivity of TNBC cells to 5-FU and doxorubicin.

\subsubsection{Combination with ARF Exchange Activity Inhibitors (ARFis)}

The ARFi brefeldin A (BFA) [549] has been demonstrated to sensitize MDA-MB-231 cells to gefitinib, leading to a synergistic increase of apoptosis [288]. In addition, BFA potentiates the PI3K inhibitor LY294002 and the SFK inhibitor PP2 but not the MEKi PD0325901. BFA increases cytotoxicity induced by the gefitinib and PP2 combination but not the gefitinib and LY294002 combination or the gefitinib and PD0325901 combination. The gefitinib + BFA + PP2 combination further reduced the levels of p-AKT, p-ERK1/2, and p-SRC in MDA-MB-231 cells. Results from siRNA-based knockdown and overexpression of ARF1 further support that the targeting ARF1 synergistically induced the gefitinibmediated apoptosis in MDA-MB-231 cells [288].

\subsubsection{Combination with Protein-Protein Interaction Inhibitors (PPIis)}

Targeting of the protein-protein interaction (PPI) to control disease has been the most challenging task. However, recent advances have resulted in approval of PPIis for marketing [550]. Disrupting the BAG3-HSP70 interaction has been demonstrated to sensitize TNBC cells to EGFRis [419]. BCL2-associated athanogene 3 (BAG3) is a cochaperone for heat shock protein 70 (HSP70) and heat shock cognate $71 \mathrm{kDa}$ (HSC70) chaperone proteins $[551,552]$. High expression levels of BAG3 mRNA and protein have been identified in approximately $50 \%$ of TNBC cell lines and clinical samples, and high mRNA levels are correlated with a poor DFS [419]. A small-molecule inhibitor of the BAG3-HSP70 interaction, YM-1 [418], enhanced the cetuximab-induced reduction of the cell viability in two TNBC cell lines, BT549 and MDA-MB-468 [419]. Similarly, knockdown of $B A G 3$ also sensitized these cells to cetuximab. Both YM-1 and BAG3-KD targeted p-AKT (S473) and p-FAK (Y397). BAG3-KD also reduced TNBC cell proliferation, migration, and invasion in vitro [419]. These results further suggest the importance of the PI3K/AKT and FAK pathways in regulating EGFRi resistance and a novel alternative strategy to overcome this issue. Exploring novel PPIis may expand the potential therapeutic options for TNBC treatment.

\subsection{Combination with Conventional Chemotherapeutics}

Conventional chemotherapeutics induce non-selective tumor cell killing and include microtubule stabilizers (vinblastine, paclitaxel, and docetaxel), DNA alkylators (cisplatin and carboplatin), anthracyclines, nucleoside analogues and nuclobases, topoisomerase inhibitors (camptothecin), and metabolic inhibitors (5-FU and methotrexate) [553].

\subsubsection{Combination with Microtubule Stabilizers}

Epothilones, which are similar to taxanes, stabilize tubulin to prevent cancer cell division [554]. Ixabepilone, a semisynthetic analog of epothiolone B [555], has been combined with gefitinib to treat TNBC cells both in vivo and in vitro. The gefitinib and ixabepilone combination reduced the growth of SUM159PT xenograft tumors compared with single treatment with both drugs [406]. Although statistical significance was not achieved, the combination also further reduced the growth of MDA-MB-231 tumors. In vitro, the 
gefitinib and ixabepilone combination reduced the autophagy markers p62 and LC3B (microtubule-associated proteins 1A/1B light chain 3B) in both TNBC cells. The effect of this combination on the mammosphere formation efficiency, both in vitro and in vivo, also did not reach statistical significance. To date, the clinical results of the combination of EGFRis and ixabepilone have shown limited efficacy (see Section 5. Clinical Studies).

\subsubsection{Combination with DNA-Damaging Agents}

DNA-damaging agents include a broad range of compounds that demonstrate anticancer activity through either covalent or non-covalent DNA binding [556].

Cisplatin (cis-diamminedichloroplatinum 2, CDDP) interacts with DNA and forms DNA adducts [557]. Cisplatin inhibits the proliferation of various cancer cells and activates DNA damage responses. The combination of cisplatin with cetuximab induced apoptotic cell death and antiproliferative effects in MDA-MB-468 cells, while cetuximab alone failed to induce apoptotic cell death [365]. This synergism demonstrated no dose response of cetuximab. In addition, the cetuximab and cisplatin treatment induced an increase in BRCA1 and a decrease in EGFR [365]. Consistent with this finding, BRCA1 has been demonstrated to downregulate EGFR by targeting its 3'-UTR by miR-146a [525]. BRCA1 transactivates miR-146a by binding to its promoter.

Anthracyclines, such as daunorubicin, doxorubicin, epirubicin, and idarubicin, are chemotherapeutics extracted from Streptomyces species [558]. The combination of cetuximab or panitumumab with cisplatin or epirubicin showed synergistic effect in decreasing cell proliferation of the BRCA1-mutated TNBC cell line SUM1315MO2, which has a wild-type KRAS and PTEN background, whereas no effect was found in another BRCA1-mutated TNBC cell line, HCC9137, with PTEN deficiency [407]. The TNBC cell lines with wtBRCA1, MDA-MB-231 (KRAS-mutated), and MDA-MB-468 (PTEN-null) also were not affected by this combination. Of interest, restoration of wtBRCA1 abolished this synergism. Anti-EGFR antibodies as a monotherapy did not affect the cell cycle distribution but DNA-damaging agents alone induced marked $G_{2}$ cell cycle arrest. The combination of anti-EGFR antibodies and DNA-damaging agents redistributes the cell cycle by reducing $G_{2}$ and increasing $G_{1}$ cell cycle arrest. No additional increase in DNA-damaging, agent-induced apoptosis was observed [407]. One plausible explanation for the differential effects of the combination found in SUM1315MO2 and HCC1937 cells is activation of the PI3K/AKT pathway, since PTEN-deficiency may cause activation of PI3K/AKT signaling in HCC1937 cells [559]. Further studies are needed to elucidate the mechanism of action for this combination.

\subsubsection{Combination with Nucleoside Analogues and Nucleobases}

Cytotoxic nucleoside analogues and nucleobases are antimetabolites that inhibit DNA or RNA synthesis through incorporation into growing DNA or RNA strands and termination of their extension [560]. These agents are used as antiviral or anticancer chemotherapeutics. These drugs include (1) the purine analogues fludarabine and cladribine, (2) the pyrimidine analogues cytarabine and gemcitabine; and (3) the fluoropyrimidines fluorouracil and capecitabine [560].

Gemcitabine is a cytidine analogue that is widely used to treat cancers such as bladder cancer [561], breast cancer [562], NSCLC [563], ovarian cancer [564,565], and metastatic pancreatic cancer [566] as a single agent or in combination with other chemotherapeutics. After entering cells, gemcitabine is converted into various nucleotides, including difluorodeoxycytidine triphosphate ( $\mathrm{dFdCTP}$ ), difluorodeoxycytidine diphosphate (dFdCDP), and difluorodeoxyuridine monophosphate (dFdUMP) [567]. These metabolites inhibit DNA polymerase (dFdCTP) [568], ribonucleotide reductase (dFdCDP) [569,570], and thymidylate synthase (dFdUMP) [571].

Gemcitabine has been reported to overcome erlotinib resistance in erlotinib-resistant, EGFR-overexpressing A431 cells by downregulating p-AKT levels [11]. Similar to this squamous carcinoma cell line, gemcitabine also reduced p-AKT in the TNBC cell lines BT549 
and MDA-MB-468 and synergistically reduced the viability of TNBC cells in combination with erlotinib [11].

\subsubsection{Combination with Multiple Chemotherapeutics}

Similar to PKIs, the anticancer effects of EGFRis in combination with multiple chemotherapeutics have been investigated. A triple combination of gefitinib, carboplatin, and docetaxel showed synergism in antiproliferation assays [366]. This triple combination was superior to gefitinib and carboplatin or gefitinib and docetaxel in antiproliferation assays. Furthermore, this triple combination induced a similar level of $\mathrm{G}_{2} / \mathrm{M}$ cell cycle arrest compared with the combination of carboplatin and docetaxel [366].

\subsection{Other Combination Strategies}

\subsubsection{Combination of Anti-EGFR Antibodies}

A strategy using combinations of non-competitive anti-EGFR antibodies has been suggested to achieve robust degradation of EGFR, leading to tumor inhibition [381]. Administration of a mixture of monoclonal antibodies (mAbs) may have resulted in synergistic effects because individual $\mathrm{mAbs}$ have partial antitumor effects based on diverse neutralizing effects, such as inhibition of ligand binding, prevention of receptor dimerization, and induction of receptor internalization. The combination of panitumumab and $\mathrm{mAb} 111$ reduced surface EGFR proteins by enhancing their internalization and degradation. Diminished surface EGFR led to reduction of TNBC cell invasion and colony formation in vitro and tumor growth in vivo [381].

A mixture of six monoclonal antibodies against EGFR, HER2, and HER3 was reported to reduce the growth of fifteen TNBC PDX tumors in vivo [446]. Treatment with this Pan-HER antibody mixture reduced the levels of p-EGFR (Y1068) and p-HER3 (Y1289) and their total protein levels, with consistent reductions in their downstream effectors p-AKT (T308), p-ERK1/2, and p-FAK (Y397) in PDX tumors in vivo. The RT-PCR-based expression analysis of 88 EGFR-associated genes demonstrated the downregulation of genes such as RAS, RAF, MEK, ERK, JNK, c-Jun, c-Fox, JAK, STAT3, IKK, NF- $k B$ p52, and p65/RelA [446]. These results are consistent with the EGFR-NF-kB crosstalk previously reported (see Section 3.3.3. Activation of the NF-kB Pathway).

\subsubsection{Combination in a Single Molecule: Bispecific Antibody}

A single-chain diabody is a recombinant multivalent and bispecific single-chain antibody fragment [572]. Recently, a tetravalent, bispecific single-chain diabody-Fc fusion protein targeting EGFR and HER3 was developed, and its anticancer efficacy in TNBC cells both in vitro and in vivo was demonstrated [445]. Since the kinase activity of HER3 is very low (see Section 3.2. Activation of Bypass Signaling Pathways), functional blocking of HER3 is achievable through antibodies binding to its ectodomain, but not with small-molecule TKIs [445]. The diabody, composed of the antigen-binding sites of a humanized cetuximab (IgG hu225) and IgG 3-43, which target EGFR and HER3, respectively, has been reported to downregulate p-EGFR (Y1068) and p-HER3 (Y1289), as well as their downstream effectors p-AKT (T308) and p-ERK (T202/Y204), in FaDu, a hypopharyngeal carcinoma cell line. The diabody was further demonstrated to reduce TNBC CSC $\left(\mathrm{ALDH}^{\mathrm{High}}\right)$ populations both in vitro and in vivo and consistently reduced TNBC tumor regrowth in vivo more efficiently than the parental antibodies alone or a combination of them [445].

\subsubsection{Combination with Autophagy Inhibitors}

As mentioned earlier, autophagy has been established as a novel mechanism and therapeutic target to overcome anticancer drug resistance [331-333]. EGFR TKIs have been reported to activate autophagy as a cytoprotective response in various cancer cell lines, including breast cancer [333], lung cancer [573,574], squamous cell carcinoma, and transitional cell carcinoma [337]. Gefitinib-induced autophagy in two TNBC cell lines, MDAMB-231 and MDA-MB-468, is inhibited by either 3-methyladenine (3-MA) or bafilomycin 
A1 (Baf-A1) [415]. Blocking the gefitinib-induced autophagy resulted in synergistic augmentation of cytotoxicity in TNBC cells in vitro and anticancer effects on TNBC tumors in vivo. The combination of gefitinib with autophagy inhibitors induced $G_{0} / G_{1}$ cell cycle arrest and accumulation of DNA damages as demonstrated by increases in the levels of phosphorylation of ataxia telangiectasia mutated (ATM), CHEK1, CHEK2, and $\gamma-\mathrm{H} 2 \mathrm{AX}$, a hallmark of DNA double-strand breaks [575]. The combination further induced apoptosis with induction of BCL2-associated X (BAX), cleaved caspase-3, and cytochrome C release, and a reduction in the anti-apoptotic protein BCL2 [415].

The combination of osimertinib and chloroquine (CHQ), an autophagy and lysosome inhibitor [576], has been reported to show anticancer effects, namely a reduction in cell viability in MDA-MB-231 cells [426]. Concordantly, LC3B-II, an autophagic marker that is tightly associated with autophagosomal membranes [577], was upregulated by this combination. Furthermore, the proapoptotic proteins, BCL2-associated agonist of cell death (BAD) [578] and active caspase-3, were increased by this combination. These results suggest that the osimertinib and CHQ combination exerts anticancer effects through autophagyapoptosis crosstalk, but further details remain to be determined [426].

Interestingly, a recent study demonstrated that blocking autophagosome clearance with the combination of gefitinib and ROCKi (GSK269962A) might induce antitumor activity in TNBC [339] (see Section 4.1.11. Combination with Rho-Associated, Coiled-CoilContaining Protein Kinase (ROCK) Inhibitors).

\subsubsection{Combination with Antioxidants}

Inhibiting ROS is a potential approach to overcome EGFRi resistance. Previously, co-treatment of genetically engineered catalase sensitized the TNBC cell line MDA-MB468 to gefitinib [373,417]. In MDA-MB-468 cells, a high level of ROS induced tyrosine phosphorylation of EGFR [373]. Transduction of genetically engineered catalase (CAT-SKL) reduced ROS-mediated EGFR phosphorylation and enhanced gefitinib cytotoxicity in two TNBC cell lines, MDA-MB-468 and SUM149PT [373]. A subsequent study demonstrated that CAT-SKL inhibits CSCs and erlotinib inhibits non-CSCs in a subset of TNBC cell lines, such as HCC70 and MDA-MB-468 cells [417]. CAT-SKL reduced the expression of methyl-CpG binding domain 2 isoform c (MBD2c), leading to a reduction of mammosphere formation. Overexpression of MBD2c in MDA-MB-468 cells increased the number and size of mammospheres. Co-treatment with the antioxidant (-) epicatechin with erlotinib confirmed the use of the CAT-SKL and erlotinib combination [417]. These results imply the importance of targeting subpopulations of heterogenous tumor cells. Further studies on the role of MBD2c in CSCs may provide new insights for TNBC therapeutics.

\subsubsection{Combination with Natural Products}

Icaritin is a natural compound from the Epimedium genus with anticancer activity [579]. The combination of cetuximab with icaritin reduced cell viability and induced apoptosis in the TNBC cell lines MDA-MB-231 and MDA-MB-436 [450]. This combination further resulted in a reduction of MDA-MB-231 and MDA-MB-436 xenograft tumor growth in vivo. Icaritin has been demonstrated to decrease both EGFR and ER $\alpha 36$ expression in TNBC cells [580]. The expression of $E R \alpha 36$ is associated with stemness and metastasis of breast cancer and chemoresistance in ER-negative breast cancer (see Section 3.4.5. Overexpression of the Estrogen Receptor Alpha (Er $\alpha$ ) Variant).

\subsubsection{Combination with Gene Therapy}

As mentioned earlier, $p 53$ mutations are commonly found in TNBC [120,192]. Since p53 mutations result in gain-of-function as an oncogene as well as loss-of-function in terms of tumor suppressor function, targeting mtp53 or restoring wild-type p53 (wtp53) is a promising therapeutic option [239]. Notably, adenoviral-vector-expressing wtp53 (Ad-wtp53) was approved as the world's first commercial gene therapy product by the Chinese regulatory authority [581]. Ad-wtp53 decreased the growth of MDA-MB-468 
xenograft tumors in combination with gefitinib [398]. Combined treatment with Ad-wtp53 and gefitinib induced $\mathrm{G}_{2} / \mathrm{M}$ cell cycle arrest and apoptosis and reduced activation of the PI3K/AKT/mTORC1 pathway. It would be an interesting research topic to evaluate whether small-molecule inhibitors of mtp53, such as the SIRT1 activator YK-3-237 [240], augment EGFRi effects in TNBC cells.

The combination of cetuximab with an miR-155-5p antagomir has been demonstrated to enhance the anticancer effects of cetuximab in MDA-MB-468 cells through promoting apoptosis and pyroptosis, both in vitro and in vivo [453]. Pyroptosis is caspase-1dependent programmed cell death and is proinflammatory in nature [582]. The common features of this combination effect in vitro and in vivo are the downregulation of p-EGFR and upregulation of gasdermin-E (GSDME). MiR-155-5p directly targets the $3^{\prime}-\mathrm{UTR}$ of GSDME mRNA [453]. Of importance, GSDME is a precursor protein of the pore-forming $\mathrm{N}$-terminus of GSDME (GSDME-N) that converts apoptosis to pyroptosis [583,584]. Upon cleavage by caspase-3, GSDME-N binds to and perforates the plasma membrane to trigger inflammatory pyroptosis [584]. These results suggest the potential importance of pyroptosis as a novel mechanism of EGFRi potentiation by drug combination.

\subsection{Immuno-Oncological Approaches}

Since TNBCs have been identified as an immunogenic malignance [585-588], immunooncological approaches may be promising alternatives for TNBC treatment. The immunooncological approaches described in the following sections have not been fully explored yet in combination with EGFRis; the future studies on EGFR targeting in TNBC will be expected to include these new modalities.

\subsubsection{ADCC}

Although cetuximab alone did not induce antiproliferative effects or apoptosis in TNBC cells in vitro, it did induce the NK-cell-dependent ADCC in vitro in EGFR-expressing TNBC cells [369]. This ADCC was further enhanced in vitro, ex vivo, and in vivo by interleukin (IL)-2 or IL-15, which stimulates NK cells to produce interferon (IFN)- $\gamma[369,370]$. The ADCC induced by the cetuximab and IL-2 or IL-15 combination was more evident, since the combination reduced the xenograft tumor volume with an increase in infiltrating NK cells, whereas no significant induction of direct antiproliferative effects on the tumor was observed [370]. A subsequent study reported that the combination of cetuximab and IL-15 further activated tumor killing by NK cells and stimulated the maturation of dendritic cells (DCs) in a co-culture experiment with the TNBC cell line IIB-BR-G [439]. Taken together, the combination of anti-EGFR antibodies and interleukin may enhance ADCC in the tumor microenvironment, even in the absence of a direct antitumor effect by anti-EGFR antibodies.

Recently, sacituzumab govitecan (Trodelvy ${ }^{\circledR}$ ) received accelerated FDA approval for the treatment of patients with metastatic TNBC [589]. Sacituzumab govitecan is an antibody drug conjugate (ADC) composed of a humanized monoclonal antibody for trophoblast cell surface antigen 2 (Trop-2) and topoisomerase inhibitor SN-38 [590]. Sacituzumab govitecan was granted regular approval by the US FDA for TNBC on 7 April 2021 [591]. As for IL-2 and IL-15, it is of interest whether the combination of EGFRis and sacituzumab govitecan is effective for TNBC or not.

\subsubsection{Chimeric Antigen Receptor (CAR)-Engineered Cell Therapies}

CAR-engineered immune cell therapy has been established as a promising immunooncologic strategy for cancer therapy. After the great success of CAR-engineered T (CART) cell therapy [592], CAR-engineered NK (CAR-NK) cells [593] and CAR-engineered macrophages (CAR-M) [594] have been developed to reinforce the treatment of multiple cancers. The major challenge of CAT-T is that the clinical benefit is limited in hematologic cancers but not in solid tumors $[595,596]$. 
Cell surface antigens, including RTKs (e.g., AXL, EGFR, MET, ROR, etc.), which are overexpressed in TNBCs, are promising targets for CAR-T cell therapy [597]. Since in vitro and in vivo studies have demonstrated the efficacy of CAR-T cell therapy against TNBC cells, combination approaches may further expand the therapeutic opportunities in the future. In fact, CD32 $\mathrm{A}^{131 \mathrm{R}}$-chimeric receptor (CR) T cells in combination with cetuximab or panitumumab resulted in in vitro anticancer activity in TNBC cells [447]. Cetuximab (IgG1) can induce ADCC- of EGFR-positive cells, whereas panitumumab (IgG2) cannot be due to distinct binding to the different $\mathrm{Fc}_{\mathrm{c}}$ gamma receptors ( $\mathrm{Fc} \gamma \mathrm{Rs}$ ) CD16 and CD32A; IgG2 has low affinity for CD16, while IgG1 and IgG2 bind to CD32A with different affinities [598,599]. The CD32A ${ }^{131 R}-C R$ T cells were superior to $C D 16^{158 F_{-}}-\mathrm{CR}$ T cells in their antitumor activity against the EGFR-positive TNBC cell line MDA-MB-468 [447]. The antitumor effects of CD32 $\mathrm{A}^{131}$-CR $\mathrm{T}$ cells in combination with anti-EGFR antibodies were dependent on the level of cell surface EGFR, since this combination does not induce cytotoxicity of MDAMB-231 cells. The level of EGFR was approximately 2.1-fold higher in MDA-MB-468 cells than in MDA-MB-231 cells [447]. Notably, recently the combination of small-molecule inhibitors and CAR-T cell therapies has been extensively studied in both preclinical and clinical settings [600-603].

\subsubsection{Immune Checkpoint Inhibitors (ICIs)}

An immune checkpoint refers to molecules acting as gatekeepers of immune responses [604]. Blocking an immune checkpoint is a promising approach to enhance antitumor immunity. Programmed cell death ligand 1 (PD-L1; also named CD127 or B7-H1) is a ligand for the PD-1 immune checkpoint receptor that inhibits the T-cell effector function in the tumor microenvironment, leading to escape of tumor immunity [605]. PD-L1 expression has been found in $20 \%$ of TNBCs and is positively regulated by the PI3K/AKT pathway [606]. The ICI atezolizumab, which is an anti-PD-L1 antibody, in combination with nab-paclitaxel has been approved by the FDA for TNBC treatment [607]. More recently, a pembrolizumab (KEYTRUDA) and chemotherapy (paclitaxel protein-bound, paclitaxel, or gemcitabine plus carboplatin) combination was approved by the US FDA for locally recurrent unresectable or metastatic TNBC [608]. Future studies on the combination of EGFRis and ICIs will be of interest.

\subsection{Use of EGFR as a Docking Protein for Targeted Drug Delivery}

Membrane receptor proteins that are highly expressed in tumors are also potential candidates for therapeutic targeting, imaging, and docking proteins for receptor-targeted delivery systems $[609,610]$. Drugs can be directly conjugated to the targeting ligand against receptor proteins or encapsulated into receptor-targeted nanocarriers [610]. Due to its high level expression on the surface of various cancers, EGFR has been established as a docking protein for enhanced delivery of therapeutic and diagnostic agents to the tumor stroma with EGFR-overexpressing cancers [248,610-615]. For example, approximately 8-80-fold higher expression of EGFR (0.16-1.5 $\times 10^{6}$ vs. $1.9 \times 10^{4}$ per cell) has been reported in breast and head and neck cancer cells compared to the normal human milk-derived cells $[616,617]$.

\subsubsection{Direct Drug Conjugates}

ADCs are composed of monoclonal antibodies directly linked to cytotoxic drugs (payloads) [618,619]. As a fast growing drug classes, currently nine ADCs are approved by the US FDA for cancer treatment [618]. As mentioned earlier, sacituzumab govitecan has been granted accelerated approval for patients with metastatic TNBC [602]. EGFR-targeting ADCs are also being developed (see the recent review [620]). In addition, beyond cytotoxic drugs, various payloads have been conjugated to EGFR-targeting molecules to make ADCs with novel mechanisms, which include photoimmunotheranostics, immunotoxins, cytolytic proteins, and immune modulating drugs (reviewed in [620]). Growing knowledge on the chemistry of the antibodies, linkers, and payloads and their impacts on clinical efficacy can provide further developments of this new modality $[618,620]$. 


\subsubsection{EGFR-Targeting Nanocarriers}

Nanocarriers are nano-sized $(10-400 \mathrm{~nm})$ particles that are able to encapsulate therapeutic or diagnostic molecules, which include lipid nanoparticles (liposomes), biocompatible polymers, surfactants, protein particles, RNA nanoparticles, and extracellular vesicles [248,610-615,621,622]. A variety of EGFR-targeting molecules have been utilized to effectively and selectively deliver nanocarriers to tumor stroma, including monoclonal antibodies, single-chain antibodies, nanobodies, affimers, affinity peptides, and oligonucleotide aptamers [623-630]. EGFR-targeting nanocarriers can deliver encapsulated drugs more efficiently via receptor-mediated endocytosis; however, escaping lysosomal degradation is a challenging issue that needs to be resolved [631-633]. Since nanocarriers are able to encapsulate and deliver insoluble small molecules, antibodies, and nucleic acids such as miRNA and mRNA, EGFR-targeting nanocarriers can provide alternative therapeutic opportunities for patients with TNBC and other EGFR-overexpressing cancers.

\section{Clinical Studies}

A relatively small number of clinical trials have been conducted on the combination of anti-EGFR therapeutics with other drugs. Interestingly, most of the clinical studies of the combinatorial approach have been performed with anti-EGFR antibodies (Table 5), in contrast to preclinical studies (Table 3). Among the 13 studies reported, 10 studies utilized anti-EGFR antibody therapeutics. As mentioned earlier, one disadvantage of anti-EGFR antibodies is that they are slowly infused intravenously over a recommended time period to avoid side effects $[38,39]$. In contrast, similar to other small-molecule PKIs, EGFR TKIs are orally bioavailable [52]. This advantage of EGFR TKIs provides convenience in designing and dosing in clinical studies and in medicating patients on a daily basis. In addition, multigeneration EGFR TKIs provide wide opportunities considering TNBC genetics or resistance to anticancer drugs.

\subsection{Anti-EGFR Antibodies in Combination Therapy in Clinical Studies of TNBC}

The benefit for patients with TNBC with the combination of cetuximab and paclitaxel was reported in a case report as early as 2007 [634]. A 62-year-old woman was diagnosed with skin metastasis of TNBC after mastectomy followed by adjuvant anthracycline-based chemotherapy and radiotherapy. Various chemotherapies with miltefosine, docetaxel, and vinorelbin + FU failed to treat the metastasized tumor. Surprisingly, a six-course treatment of paclitaxel + cetuximab improved the infiltrating skin metastases [634]. However, the follow-up study failed due to the patient's return to her country.

Combination treatment with cetuximab and carboplatin for patients with TNBC showed limited outcomes in a phase 2 clinical trial [635]. Compared to the cetuximab monotherapy, cetuximab + carboplatin failed to improve the time to progression (TPP) or OS, with an overall response rate (ORR) $<20 \%$ (Table 5)

In addition, unlike the in vitro study [365], a phase 2 clinical study of cetuximab + cisplatin versus cisplatin for patients with TNBC did not meet the primary end point, which was an ORR $>20 \%$ [636]. However, cetuximab + cisplatin resulted in a longer PFS and OS (Table 5).

A pilot phase 2 study of a cetuximab and docetaxel combination as a neoadjuvant therapy in stage 2 TNBC showed modest efficacy with manageable toxicity [637]. The pathologic complete response (pCR) rate was $24 \%$ and the complete clinical response (cCR) rate was $22 \%$ from 25 assessable patients.

In a randomized phase 2 clinical study, an antimicrotubule agent ixabepilone and the combination of ixabepilone and cetuximab resulted in similar clinical activities in the firstline treatment of patients with advanced TNBC [638]. These results did not confirm those of a preclinical study that showed the antitumor efficacy of the gefitinib and ixabepilone combination in the SUM159PT xenografts [406].

Another phase 2 clinical trial involved single-arm treatment with the combination of cetuximab and irinotecan [639]. Among 19 patients, 58\% had TNBC. The response rate (RR) of TNBC was $18 \%$, whereas that of non-TNBC was $0 \%$. Although the study was terminated 
early due to the low RR and rapid disease progression, potentially promising results were noted in patients with TNBC.

Another single-arm study involved the combination of panitumumab with 5-FU + epidoxorubicin + cyclophosphamide (FEC) followed by the combination of panitumumab with docetaxel [640]. The results showed that the treatment was efficacious, with acceptable toxicity. Among 47 assessable patients, the CR was 59.6\% (28). A high CD8+ tumorinfiltrating lymphocyte (TIL) count was found to be the strongest predictor for pCR in this study.

A single-arm phase 2 clinical study evaluated a triple combination of the anti-EGFR antibody panitumumab with paclitaxel and carboplatin and showed limited efficacy due to side effects, including neutropenia and thrombocytopenia [641]. Among 14 assessable patients, the ORR was $46 \%$ and 2 patients achieved CR after 6 and 9 cycles of therapy. Prolonged neutropenia and thrombocytopenia limited the intended dosing. Similar doselimiting toxicities have been identified with other EGFRis and platinum-based chemotherapy combinations in patients with NSCLC and metastatic breast cancer [642-644].

A single-arm phase 2 study of the combination of panitumumab and neoadjuvant chemotherapy showed the highest PCR rate ever reported in patients with TNBC; however, 10 patients were hospitalized due to treatment-related toxic effects, such as neutropenia, diarrhea, pulmonary embolism, bleeding from the rectum, fever without neutropenia, and confusion of unknown origin [645]. The treatment scheme was relatively complex (Table 5). The expression of p-EGFR and cyclooxygenase-2 (COX2) was correlated with pCR, while no correlations were observed in the expressions of EGFR, E-cadherin, vimentin, and nodal. RNA sequencing analysis revealed the 2 downregulated (POU3F3 and EGF1) and 4 upregulated (BBOX1, GLYATL2, MUCL1, and LCN2) genes in samples from patients with TNBC after the first dose of panitumumab treatment. A randomized phase 2 clinical trial (NCT02876107) of the same chemotherapy with and without panitumumab in patients with TNBC is currently under way.

A single-arm phase 2 trial of the combination of panitumumab, gemcitabine, and carboplatin showed no beneficial effect in patients with TNBC [646]. The primary endpoint of median PFS (4.4 months) did not reach the prespecified PFS (5.5 months). No correlation between EGFR amplification, aberrant PI3K pathway activation, or p53 expression and response to this combination has been found.

\subsection{EGFR TKIs in Combination Therapy in Clinical Studies of TNBC}

A randomized phase 2 clinical study of gefitinib with neoadjuvant epirubicin and cyclophosphamide (EC) in estrogen-negative breast cancer was not successful. However, post hoc analysis revealed that a significantly higher $\mathrm{pCR}$ rate was observed in the TNBC $(7 / 41,17 \%)$ than in the non-TNBC $(1 / 48,2 \%)$ subgroups, but no significant difference was observed between combination therapy (7/41) and EC (5/41) [647].

A phase 1 study of the erlotinib and metformin combination was conducted in 8 patients with TNBC [649]. with a fixed dose of erlotinib of $150 \mathrm{mg}$ daily, a $3+3$ design of metformin dose escalation was evaluated to determine the dose-limiting toxicities (DLTs). Although no DLTs were observed, no efficacy was demonstrated.

An open-label pilot study of the lapatinib and veliparib combination demonstrated the potential antitumor activity of this combination in patients with advanced TNBC without DLTs [648]. Gene expression analysis revealed an increase in the expression of genes involved in antigen presentation, immune cell infiltration, and cytokine and chemokine signaling. As expected, a reduction was observed in the DNA damage repair genes, such as $B R C A 1, C R Y A B$, and $C K B$. Interestingly, the lapatinib and veliparib combination was reported to have synergistic efficacy in reducing colony formation of TNBC cell lines in vitro [372]. 
Table 5. Clinical trials of EGFR combination therapy for patients with TNBC.

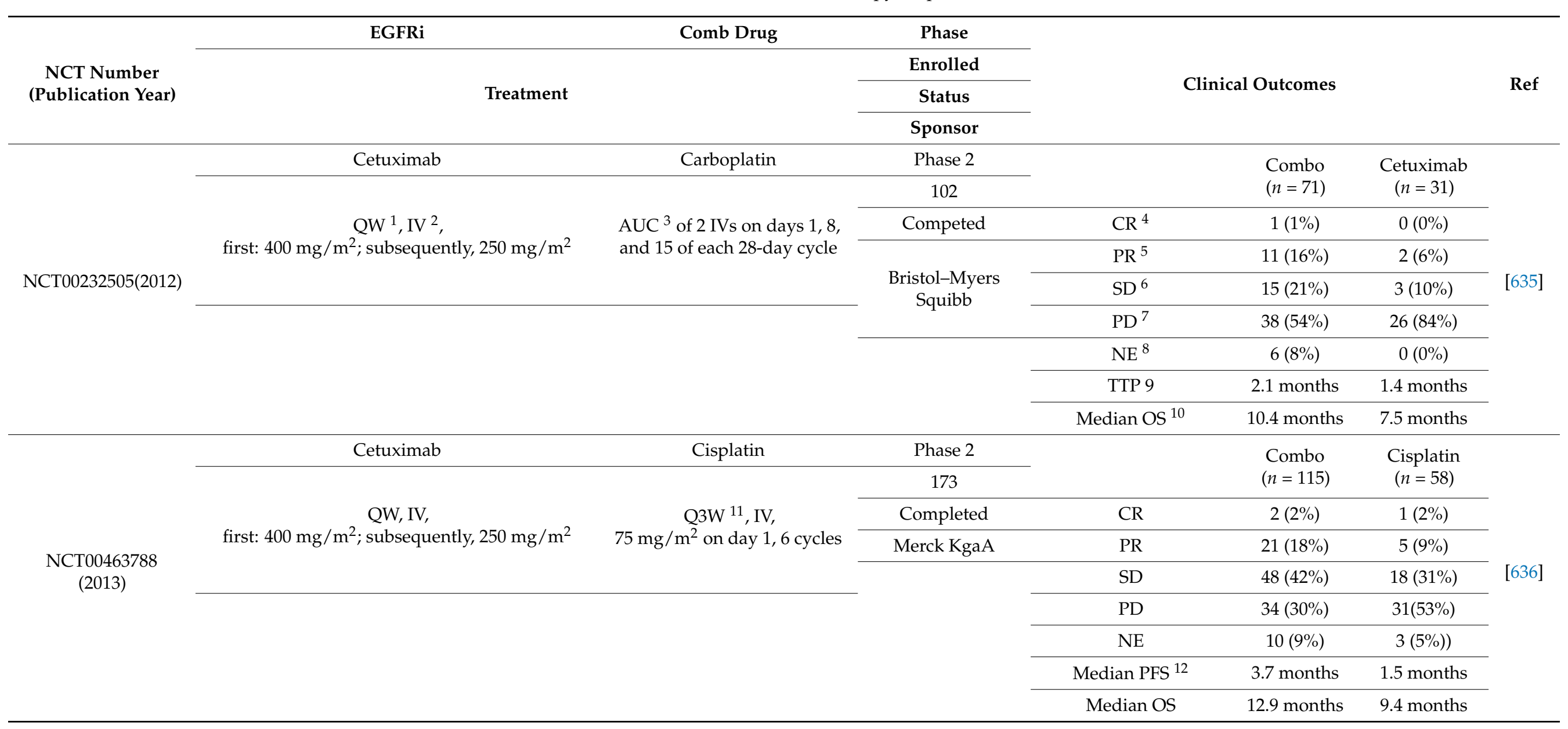


Table 5. Cont

\begin{tabular}{|c|c|c|c|c|c|c|c|}
\hline \multirow{4}{*}{$\begin{array}{c}\text { NCT Number } \\
\text { (Publication Year) }\end{array}$} & EGFRi & Comb Drug & Phase & & & & \multirow{4}{*}{ Ref } \\
\hline & \multirow{3}{*}{\multicolumn{2}{|c|}{ Treatment }} & Enrolled & \multirow{3}{*}{\multicolumn{3}{|c|}{ Clinical Outcomes }} & \\
\hline & & & Status & & & & \\
\hline & & & Sponsor & & & & \\
\hline \multirow{5}{*}{$\begin{array}{l}\text { NCT00600249 } \\
(2016)\end{array}$} & Cetuximab & Docetaxel & \multirow{2}{*}{ Phase 2} & & \multirow{2}{*}{\multicolumn{2}{|c|}{$\begin{array}{l}\text { Combo } \\
(n=25)\end{array}$}} & \multirow{5}{*}{ [637] } \\
\hline & & \multirow{4}{*}{ Q3W, $\left(100 \mathrm{mg} / \mathrm{m}^{2}\right)$ on day 1,6 cycles } & & & & & \\
\hline & first: $400 \mathrm{mg} / \mathrm{m}^{2}$; & & 25 & & & & \\
\hline & subsequently: & & Completed & $\mathrm{pCR}$ & & & \\
\hline & & & $\begin{array}{c}\text { Merch Serono and } \\
\text { Sanofi-Aventis }\end{array}$ & $\mathrm{cCR}^{13}$ & \multicolumn{2}{|c|}{$22 \%$} & \\
\hline \multirow{8}{*}{$\begin{array}{l}\text { NCT00633464 } \\
(2015)\end{array}$} & Cetuximab & Ixabepilone & Phase 2 & & \multirow{2}{*}{$\begin{array}{l}\text { Combo } \\
(n=39)\end{array}$} & \multirow{2}{*}{$\begin{array}{l}\text { Ixabepilone } \\
\quad(n=40)\end{array}$} & \multirow{8}{*}{ [638] } \\
\hline & & \multirow{3}{*}{$\mathrm{Q} 3 \mathrm{~W}, \mathrm{IV}, 400 \mathrm{mg} / \mathrm{m}^{2}$} & 79 & & & & \\
\hline & first: $400 \mathrm{mg} / \mathrm{m}^{2}$ & & Completed & $\mathrm{CR}$ & 0 & $3(7.5 \%)$ & \\
\hline & subsequently, & & \multirow{2}{*}{$\begin{array}{l}\text { Bristol-Myers } \\
\text { Squibb }\end{array}$} & PR & $14(35.9 \%)$ & $9(22.5 \%)$ & \\
\hline & & & & $\mathrm{PD}$ & $10(25.6 \%)$ & $9(22.5 \%)$ & \\
\hline & & & & $\mathrm{NE}$ & $1(2.6 \%)$ & $2(5.0 \%)$ & \\
\hline & & & & ORR & $14(35.95)$ & $12(30.0 \%)$ & \\
\hline & & & & Median PFS & 4.1 months & 4.1 months & \\
\hline
\end{tabular}


Table 5. Cont.

\begin{tabular}{|c|c|c|c|c|c|c|c|}
\hline \multirow{3}{*}{$\begin{array}{c}\text { NCT Number } \\
\text { (Publication Year) }\end{array}$} & \multicolumn{3}{|r|}{ Comb Drug } & Phase & \multirow{3}{*}{\multicolumn{2}{|c|}{ Clinical Outcomes }} & \multirow{3}{*}{ Ref } \\
\hline & \multirow{3}{*}{\multicolumn{3}{|c|}{ Treatment }} & Enrolled & & & \\
\hline & & & & Status & & & \\
\hline \multirow{10}{*}{$\begin{array}{l}\text { NCT00275041 } \\
\quad(2016)\end{array}$} & & & & \multirow{3}{*}{ Phase 2} & & \multirow{3}{*}{$\begin{array}{l}\text { Combo } \\
(n=19)\end{array}$} & \multirow{10}{*}[639]{} \\
\hline & Cetuximab & & notecan & & & & \\
\hline & OW IV & \multirow{4}{*}{\multicolumn{2}{|c|}{$\mathrm{IV}, 80 \mathrm{mg} / \mathrm{m}^{2}$ on days 1 and 8 of a 21 -day cycle. }} & & & & \\
\hline & first: $400 \mathrm{mg} / \mathrm{m}^{2}$; & & & 19 & & & \\
\hline & subsequently & & & Completed & $\mathrm{CR}$ & 1 & \\
\hline & & & & \multirow{5}{*}{$\begin{array}{l}\text { NCI and Alliance } \\
\text { for Clinical Trials } \\
\text { in Oncology }\end{array}$} & PR & 1 & \\
\hline & & & & & ORR & $11 \%$ & \\
\hline & & & & & RR & $\begin{array}{c}\text { TNBC, } 18 \% \text { vs. non-TNBC, } \\
0 \%\end{array}$ & \\
\hline & & & & & Median OS & 9.4 months & \\
\hline & & & & & Median TTP & 1.4 months & \\
\hline \multirow{6}{*}{$\begin{array}{l}\text { NCT00933517 } \\
\quad(2014)\end{array}$} & \multicolumn{2}{|c|}{ Panitumumab } & $\begin{array}{l}\text { FEC }^{14} \text { and } \\
\text { docetaxel }\end{array}$ & 62 & & $\begin{array}{l}\text { Combo } \\
(n=47)\end{array}$ & \multirow{6}{*}{ [640] } \\
\hline & \multirow{5}{*}{\multicolumn{2}{|c|}{$\begin{array}{c}\text { Q3W, IV, } \\
9 \mathrm{mg} / \mathrm{kg}, 8 \text { cycles }\end{array}$}} & \multirow{5}{*}{$\begin{array}{c}\text { Q3W, IV, FEC: } \\
\text { 500/100/500 mg } / \mathrm{m}^{2}, 4 \text { cycles } \\
\text { followed by Q3W, IV } \\
\text { docetaxel: } 100 \mathrm{mg} / \mathrm{m}^{2}, \\
4 \text { cycles }\end{array}$} & Completed & CR & $28(59.6 \%)$ & \\
\hline & & & & \multirow{4}{*}{ Centre Jean Perrin } & PR & $3(6.4 \%)$ & \\
\hline & & & & & SD & $3(6.4 \%)$ & \\
\hline & & & & & Progression & $2(4.3)$ & \\
\hline & & & & & NE & $11(23.4)$ & \\
\hline
\end{tabular}


Table 5. Cont.

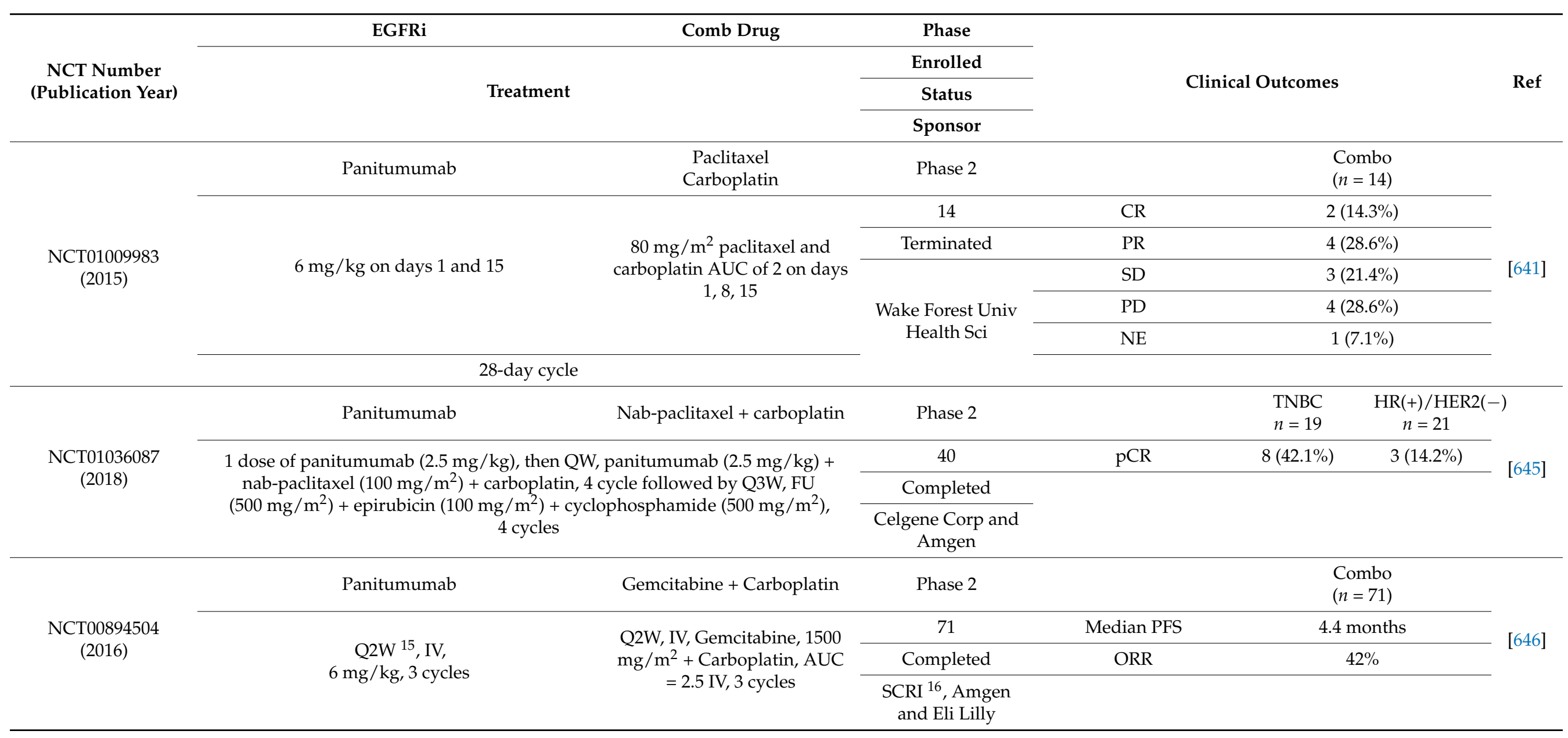


Table 5. Cont

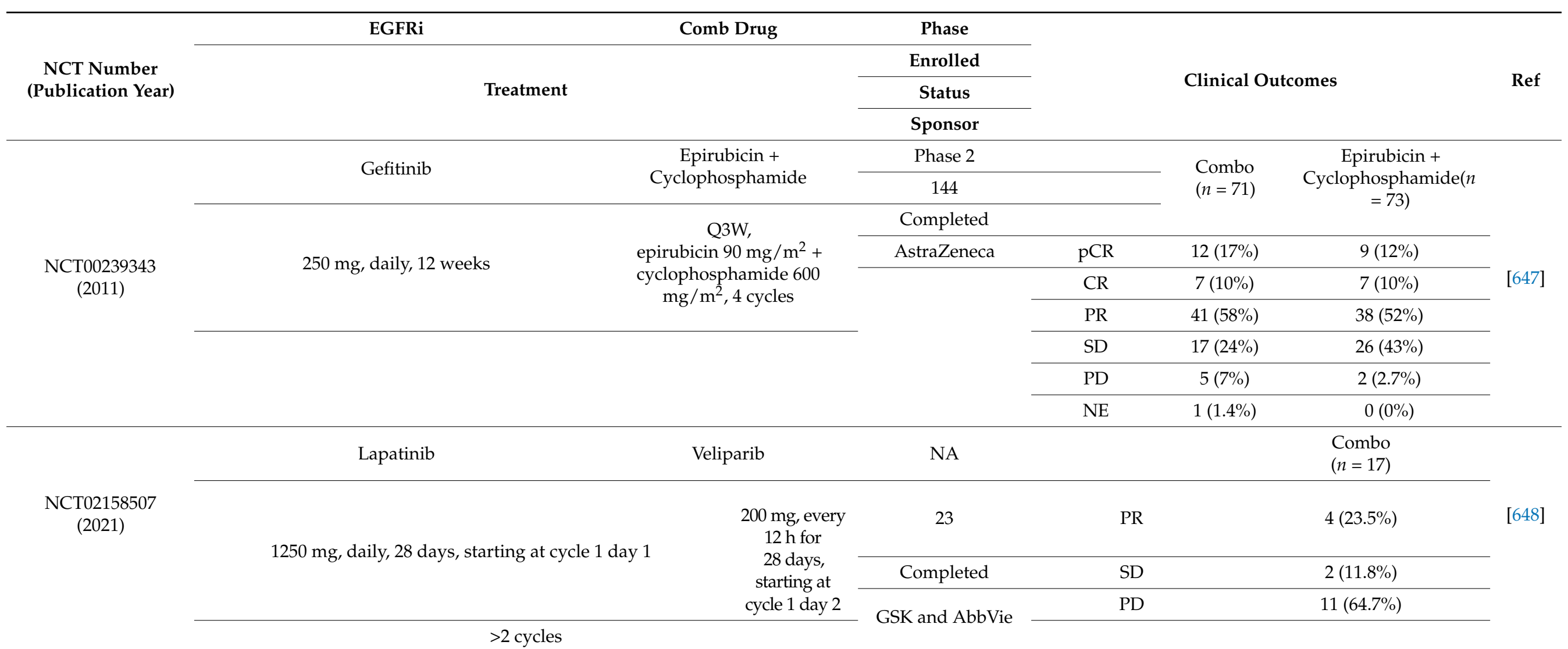

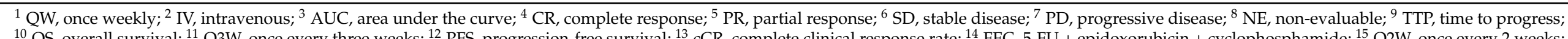

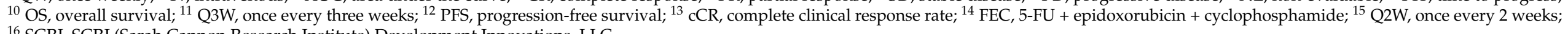

${ }^{16}$ SCRI, SCRI (Sarah Cannon Research Institute) Development Innovations, LLC. 


\section{Conclusions}

A wide variety of EGFRi combinations have been successfully applied in TNBC cells preclinically, both in vitro and in vivo (Table 3); however, most of them have not been evaluated clinically yet. To date, no appreciable success of EGFRi combinations has been found in clinical trials (Table 5). Further investigation of the potential of EGFRi combinations should be performed to develop better therapeutics for TNBC in the future.

Author Contributions: Conceptualization, investigation, and writing, K.S.Y., Y.W.Y., J.C., J.-S.P. and Y.-S.S.; funding acquisition, J.C. and Y.-S.S. All authors have read and agreed to the published version of the manuscript.

Funding: This work was supported by the National Research Foundation of Korea (NRF) grants funded by the Korea Mistry of Science and ICT (MSIT): NRF-2015R1D1A1A01057893 (Y.-S.S), NRF 2018R1D1A1B070489 (Y.-S.S), and NRF-2019R1A4A1028268 (J.C and Y.-S.S).

Institutional Review Board Statement: Not applicable.

Informed Consent Statement: Not applicable.

Data Availability Statement: Not applicable.

Conflicts of Interest: The authors declare no conflict of interest.

\section{References}

1. Robinson, D.R.; Wu, Y.-M.; Lin, S.-F. The Protein Tyrosine Kinase Family of the Human Genome. Oncogene 2000, 19 , 5548-5557. [CrossRef] [PubMed]

2. Wieduwilt, M.J.; Moasser, M.M. The Epidermal Growth Factor Receptor Family: Biology Driving Targeted Therapeutics. Cell Mol. Life Sci. 2008, 65, 1566-1584. [CrossRef] [PubMed]

3. Wheeler, D.L.; Dunn, E.F.; Harari, P.M. Understanding Resistance to EGFR Inhibitors-Impact on Future Treatment Strategies. Nat. Rev. Clin. Oncol. 2010, 7, 493-507. [CrossRef] [PubMed]

4. Eccles, S.A. The Epidermal Growth Factor Receptor/Erb-B/HER Family in Normal and Malignant Breast Biology. Int. J. Dev. Biol. 2011, 55, 685-696. [CrossRef]

5. Yarden, Y.; Pines, G. The ERBB Network: At Last, Cancer Therapy Meets Systems Biology. Nat. Rev. Cancer 2012, 12, 553-563. [CrossRef] [PubMed]

6. Scaltriti, M.; Baselga, J. The Epidermal Growth Factor Receptor Pathway: A Model for Targeted Therapy. Clin. Cancer Res. 2006, 12, 5268-5272. [CrossRef] [PubMed]

7. Yarden, Y.; Sliwkowski, M.X. Untangling the ErbB Signalling Network. Nat. Rev. Mol. Cell Biol. 2001, 2, 127-137. [CrossRef]

8. Wee, P.; Wang, Z. Epidermal Growth Factor Receptor Cell Proliferation Signaling Pathways. Cancers 2017, 9, 52. [CrossRef]

9. Zulkifli, A.A.; Tan, F.H.; Putoczki, T.L.; Stylli, S.S.; Luwor, R.B. STAT3 Signaling Mediates Tumour Resistance to EGFR Targeted Therapeutics. Mol. Cell Endocrinol. 2017, 451, 15-23. [CrossRef]

10. Normanno, N.; Bianco, C.; Luca, A.D.; Maiello, M.R.; Salomon, D.S. Target-Based Agents against ErbB Receptors and Their Ligands: A Novel Approach to Cancer Treatment. Endocr. Relat. Cancer 2003, 10, 1-21. [CrossRef]

11. Bartholomeusz, C.; Yamasaki, F.; Saso, H.; Kurisu, K.; Horto-Bagyi, G.N.; Ueno, N.T. Gemcitabine Overcomes Erlotinib Resistance in EGFR-Overexpressing Cancer Cells through Downregulation of Akt. J. Cancer 2011, 435. [CrossRef]

12. Knebel, F.H.; Bettoni, F.; Shimada, A.K.; Cruz, M.; Alessi, J.V.; Negrão, M.V.; Reis, L.F.L.; Katz, A.; Camargo, A.A. Sequential Liquid Biopsies Reveal Dynamic Alterations of EGFR Driver Mutations and Indicate EGFR Amplification as a New Mechanism of Resistance to Osimertinib in NSCLC. Lung Cancer 2017, 108, 238-241. [CrossRef]

13. Xu, M.; Xie, Y.; Ni, S.; Liu, H. The Latest Therapeutic Strategies after Resistance to First Generation Epidermal Growth Factor Receptor Tyrosine Kinase Inhibitors (EGFR TKIs) in Patients with Non-Small Cell Lung Cancer (NSCLC). Ann. Transl. Med. 2015, 3, 96. [CrossRef]

14. Patel, H.; Pawara, R.; Ansari, A.; Surana, S. Recent Updates on Third Generation EGFR Inhibitors and Emergence of Fourth Generation EGFR Inhibitors to Combat C797S Resistance. Eur. J. Med. Chem. 2017, 142, 32-47. [CrossRef] [PubMed]

15. Yi, Y.W.; You, K.; Bae, E.J.; Kwak, S.-J.; Seong, Y.-S.; Bae, I. Dual Inhibition of EGFR and MET Induces Synthetic Lethality in Triple-Negative Breast Cancer Cells through Downregulation of Ribosomal Protein S6. Int. J. Oncol. 2015, 47, 122-132. [CrossRef] [PubMed]

16. Diaz, L.K.; Cryns, V.L.; Symmans, W.F.; Sneige, N. Triple Negative Breast Carcinoma and the Basal Phenotype\&colon; From Expression Profiling to Clinical Practice. Adv. Anat. Pathol. 2007, 14, 419-430. [CrossRef]

17. Huynh, M.; Pambid, M.R.; Jayanthan, A.; Dorr, A.; Los, G.; Dunn, S.E. The Dawn of Targeted Therapies for Triple Negative Breast Cancer (TNBC): A Snapshot of Investigational Drugs in Phase I and II Trials. Expert Opin. Inv. Drug 2020, 29, 1199-1208. [CrossRef] 
18. Brenton, J.D.; Carey, L.A.; Ahmed, A.A.; Caldas, C. Molecular Classification and Molecular Forecasting of Breast Cancer: Ready for Clinical Application? J. Clin. Oncol. 2005, 23, 7350-7360. [CrossRef]

19. Lehmann, B.D.; Bauer, J.A.; Chen, X.; Sanders, M.E.; Chakravarthy, A.B.; Shyr, Y.; Pietenpol, J.A. Identification of Human Triple-Negative Breast Cancer Subtypes and Preclinical Models for Selection of Targeted Therapies. J. Clin. Invest. 2011, 121, 2750-2767. [CrossRef]

20. Lehmann, B.D.; Pietenpol, J.A. Identification and Use of Biomarkers in Treatment Strategies for Triple-negative Breast Cancer Subtypes. J. Pathol. 2014, 232, 142-150. [CrossRef] [PubMed]

21. Nakai, K.; Hung, M.-C.; Yamaguchi, H. A Perspective on Anti-EGFR Therapies Targeting Triple-Negative Breast Cancer. Am. J. Cancer Res. 2016, 6, 1609-1623.

22. You, K.S.; Yi, Y.W.; Cho, J.; Seong, Y.-S. Dual Inhibition of AKT and MEK Pathways Potentiates the Anti-Cancer Effect of Gefitinib in Triple-Negative Breast Cancer Cells. Cancers 2021, 13, 1205. [CrossRef]

23. Arteaga, C.L.; Truica, C.I. Challenges in the Development of Anti-Epidermal Growth Factor Receptor Therapies in Breast Cancer. Semin. Oncol. 2004, 31, 3-8. [CrossRef]

24. Liu, D.; He, J.; Yuan, Z.; Wang, S.; Peng, R.; Shi, Y.; Teng, X.; Qin, T. EGFR Expression Correlates with Decreased Disease-Free Survival in Triple-Negative Breast Cancer: A Retrospective Analysis Based on a Tissue Microarray. Med. Oncol. 2012, 29 , 401-405. [CrossRef]

25. Nogi, H.; Kobayashi, T.; Suzuki, M.; Tabei, I.; Kawase, K.; Toriumi, Y.; Uchida, H.; Fukushima, K. EGFR as Paradoxical Predictor of Chemosensitivity and Outcome among Triple-Negative Breast Cancer. Oncol. Rep. 2009, 21, 413-417. [CrossRef] [PubMed]

26. Lehmann, B.D.; Pietenpol, J.A.; Tan, A.R. Triple-Negative Breast Cancer: Molecular Subtypes and New Targets for Therapy. Am. Soc. Clin. Oncol. Educ. Book 2015, e31-e39. [CrossRef]

27. Lehmann, B.D.; Jovanović, B.; Chen, X.; Estrada, M.V.; Johnson, K.N.; Shyr, Y.; Moses, H.L.; Sanders, M.E.; Pietenpol, J.A. Refinement of Triple-Negative Breast Cancer Molecular Subtypes: Implications for Neoadjuvant Chemotherapy Selection. PLoS ONE 2016, 11, e0157368. [CrossRef] [PubMed]

28. Ezenwajiaku, N.; Ma, C.X.; Ademuyiwa, F.O. Updates on Molecular Classification of Triple Negative Breast Cancer. Curr. Breast Cancer Rep. 2018, 10, 289-295. [CrossRef]

29. Garrido-Castro, A.C.; Lin, N.U.; Polyak, K. Insights into Molecular Classifications of Triple-Negative Breast Cancer: Improving Patient Selection for Treatment. Cancer Discov. 2019, 9, 176-198. [CrossRef]

30. Yin, L.; Duan, J.-J.; Bian, X.-W.; Yu, S. Triple-Negative Breast Cancer Molecular Subtyping and Treatment Progress. Breast Cancer Res. 2020, 22, 61. [CrossRef] [PubMed]

31. Costa, R.; Shah, A.N.; Santa-Maria, C.A.; Cruz, M.R.; Mahalingam, D.; Carneiro, B.A.; Chae, Y.K.; Cristofanilli, M.; Gradishar, W.J.; Giles, F.J. Targeting Epidermal Growth Factor Receptor in Triple Negative Breast Cancer: New Discoveries and Practical Insights for Drug Development. Cancer Treat. Rev. 2017, 53, 111-119. [CrossRef] [PubMed]

32. Tan, A.R.; Yang, X.; Hewitt, S.M.; Berman, A.; Lepper, E.R.; Sparreboom, A.; Parr, A.L.; Figg, W.D.; Chow, C.; Steinberg, S.M.; et al. Evaluation of Biologic End Points and Pharmacokinetics in Patients with Metastatic Breast Cancer After Treatment with Erlotinib, an Epidermal Growth Factor Receptor Tyrosine Kinase Inhibitor. J. Clin. Oncol. 2004, 22, 3080-3090. [CrossRef]

33. Baselga, J.; Albanell, J.; Ruiz, A.; Lluch, A.; Gascón, P.; Guillém, V.; González, S.; Sauleda, S.; Marimón, I.; Tabernero, J.M.; et al. Phase II and Tumor Pharmacodynamic Study of Gefitinib in Patients with Advanced Breast Cancer. J. Clin. Oncol. 2005, 23, 5323-5333. [CrossRef] [PubMed]

34. Modi, S.; D'Andrea, G.; Norton, L.; Yao, T.J.; Caravelli, J.; Rosen, P.P.; Hudis, C.; Seidman, A.D. A Phase I Study of Cetuximab/Paclitaxel in Patients with Advanced-Stage Breast Cancer. Clin. Breast Cancer 2006, 7, 270-277. [CrossRef] [PubMed]

35. Dickler, M.N.; Cobleigh, M.A.; Miller, K.D.; Klein, P.M.; Winer, E.P. Efficacy and Safety of Erlotinib in Patients with Locally Advanced or Metastatic Breast Cancer. Breast Cancer Res. Treat. 2009, 115, 115-121. [CrossRef]

36. Normanno, N.; Tejpar, S.; Morgillo, F.; Luca, A.D.; Cutsem, E.V.; Ciardiello, F. Implications for KRAS Status and EGFR-Targeted Therapies in Metastatic CRC. Nat. Rev. Clin. Oncol. 2009, 6, 519-527. [CrossRef]

37. Cai, W.-Q.; Zeng, L.-S.; Wang, L.-F.; Wang, Y.-Y.; Cheng, J.-T.; Zhang, Y.; Han, Z.-W.; Zhou, Y.; Huang, S.-L.; Wang, X.-W.; et al. The Latest Battles Between EGFR Monoclonal Antibodies and Resistant Tumor Cells. Front. Oncol. 2020, 10, 1249. [CrossRef]

38. Yazdi, M.H.; Faramarzi, M.A.; Nikfar, S.; Abdollahi, M. A Comprehensive Review of Clinical Trials on EGFR Inhibitors Such as Cetuximab and Panitumumab as Monotherapy and in Combination for Treatment of Metastatic Colorectal Cancer. Avicenna J. Med. Biotechnol. 2015, 7, 134-144.

39. Fala, L.; Writer, M. Portrazza (Necitumumab), an IgG1 Monoclonal Antibody, FDA Approved for Advanced Squamous NonSmall-Cell Lung Cancer. Am. Health Drug Benefits 2016, 9, 119-122.

40. Goldstein, N.I.; Prewett, M.; Zuklys, K.; Rockwell, P.; Mendelsohn, J. Biological Efficacy of a Chimeric Antibody to the Epidermal Growth Factor Receptor in a Human Tumor Xenograft Model. Clin. Cancer Res. 1995, 1, 1311-1318.

41. Cho, J. Mechanistic Insights into Differential Requirement of Receptor Dimerization for Oncogenic Activation of Mutant EGFR and Its Clinical Perspective. BMB Rep. 2020, 53, 133-141. [CrossRef] [PubMed]

42. Mazorra, Z.; Chao, L.; Lavastida, A.; Sanchez, B.; Ramos, M.; Iznaga, N.; Crombet, T. Nimotuzumab: Beyond the EGFR Signaling Cascade Inhibition. Semin. Oncol. 2018, 45, 18-26. [CrossRef] [PubMed] 
43. Giusti, R.M.; Shastri, K.; Pilaro, A.M.; Fuchs, C.; Cordoba-Rodriguez, R.; Koti, K.; Rothmann, M.; Men, A.Y.; Zhao, H.; Hughes, M.; et al. U.S. Food and Drug Administration Approval: Panitumumab for Epidermal Growth Factor ReceptorExpressing Metastatic Colorectal Carcinoma with Progression Following Fluoropyrimidine-, Oxaliplatin-, and IrinotecanContaining Chemotherapy Regimens. Clin. Cancer Res. 2008, 14, 1296-1302. [CrossRef]

44. Yang, X.-D.; Jia, X.-C.; Corvalan, J.R.F.; Wang, P.; Davis, C.G. Development of ABX-EGF, a Fully Human Anti-EGF Receptor Monoclonal Antibody, for Cancer Therapy. Crit Rev. Oncol. Hemat. 2001, 38, 17-23. [CrossRef]

45. Ramakrishnan, M.S.; Eswaraiah, A.; Crombet, T.; Piedra, P.; Saurez, G.; Iyer, H.; Arvind, A.S. Nimotuzumab, a Promising Therapeutic Monoclonal for Treatment of Tumors of Epithelial Origin. Mabs 2014, 1, 41-48. [CrossRef]

46. Talavera, A.; Friemann, R.; Gómez-Puerta, S.; Martinez-Fleites, C.; Garrido, G.; Rabasa, A.; López-Requena, A.; Pupo, A.; Johansen, R.F.; Sánchez, O.; et al. Nimotuzumab, an Antitumor Antibody That Targets the Epidermal Growth Factor Receptor, Blocks Ligand Binding While Permitting the Active Receptor Conformation. Cancer Res. 2009, 69, 5851-5859. [CrossRef]

47. Yang, X.D.; Jia, X.C.; Corvalan, J.R.; Wang, P.; Davis, C.G.; Jakobovits, A. Eradication of Established Tumors by a Fully Human Monoclonal Antibody to the Epidermal Growth Factor Receptor without Concomitant Chemotherapy. Cancer Res. 1999, 59, 1236-1243. [PubMed]

48. Bagchi, A.; Haidar, J.N.; Eastman, S.W.; Vieth, M.; Topper, M.; Iacolina, M.D.; Walker, J.M.; Forest, A.; Shen, Y.; Novosiadly, R.D.; et al. Molecular Basis for Necitumumab Inhibition of EGFR Variants Associated with Acquired Cetuximab Resistance. Mol. Cancer 2017, 17. [CrossRef] [PubMed]

49. Roskoski, R. Properties of FDA-Approved Small Molecule Protein Kinase Inhibitors: A 2021 Update. Pharm. Res. 2021, 105463. [CrossRef] [PubMed]

50. Karachaliou, N.; Fernandez-Bruno, M.; Bracht, J.W.P.; Rosell, R. EGFR First- and Second-Generation TKIs-There Is Still Place for Them in EGFR -Mutant NSCLC Patients. Transl. Cancer Res. 2018, 1, S23-S47. [CrossRef]

51. Reardon, D.A.; Wen, P.Y.; Mellinghoff, I.K. Targeted Molecular Therapies against Epidermal Growth Factor Receptor: Past Experiences and Challenges. Neuro Oncol. 2014, 16, viii7-viii13. [CrossRef]

52. Peters, S.; Zimmermann, S.; Adjei, A.A. Oral Epidermal Growth Factor Receptor Tyrosine Kinase Inhibitors for the Treatment of Non-Small Cell Lung Cancer: Comparative Pharmacokinetics and Drug-Drug Interactions. Cancer Treat. Rev. 2014, 40, 917-926. [CrossRef]

53. Kazandjian, D.; Blumenthal, G.M.; Yuan, W.; He, K.; Keegan, P.; Pazdur, R. FDA Approval of Gefitinib for the Treatment of Patients with Metastatic EGFR Mutation-Positive Non-Small Cell Lung Cancer. Clin. Cancer Res. 2016, 22, 1307-1312. [CrossRef]

54. Mitsudomi, T.; Morita, S.; Yatabe, Y.; Negoro, S.; Okamoto, I.; Tsurutani, J.; Seto, T.; Satouchi, M.; Tada, H.; Hirashima, T.; et al Gefitinib versus Cisplatin plus Docetaxel in Patients with Non-Small-Cell Lung Cancer Harbouring Mutations of the Epidermal Growth Factor Receptor (WJTOG3405): An Open Label, Randomised Phase 3 Trial. Lancet Oncol. 2010, 11, 121-128. [CrossRef]

55. Fukuoka, M.; Wu, Y.-L.; Thongprasert, S.; Sunpaweravong, P.; Leong, S.-S.; Sriuranpong, V.; Chao, T.-Y.; Nakagawa, K.; Chu, D.-T.; Saijo, N.; et al. Biomarker Analyses and Final Overall Survival Results from a Phase III, Randomized, Open-Label, First-Line Study of Gefitinib Versus Carboplatin/Paclitaxel in Clinically Selected Patients with Advanced Non-Small-Cell Lung Cancer in Asia (IPASS). J. Clin. Oncol. 2011, 29, 2866-2874. [CrossRef]

56. Inoue, A.; Kobayashi, K.; Maemondo, M.; Sugawara, S.; Oizumi, S.; Isobe, H.; Gemma, A.; Harada, M.; Yoshizawa, H.; Kinoshita, I.; et al. Updated Overall Survival Results from a Randomized Phase III Trial Comparing Gefitinib with Carboplatin-Paclitaxel for Chemo-Naïve Non-Small Cell Lung Cancer with Sensitive EGFR Gene Mutations (NEJ002). Ann. Oncol. 2013, 24, 54-59. [CrossRef]

57. Douillard, J.-Y.; Ostoros, G.; Cobo, M.; Ciuleanu, T.; McCormack, R.; Webster, A.; Milenkova, T. First-Line Gefitinib in Caucasian EGFR Mutation-Positive NSCLC Patients: A Phase-IV, Open-Label, Single-Arm Study. Br. J. Cancer 2014, 110, 55-62. [CrossRef]

58. Moyer, J.D.; Barbacci, E.G.; Iwata, K.K.; Arnold, L.; Boman, B.; Cunningham, A.; DiOrio, C.; Doty, J.; Morin, M.J.; Moyer, M.P.; et al. Induction of Apoptosis and Cell Cycle Arrest by CP-358,774, an Inhibitor of Epidermal Growth Factor Receptor Tyrosine Kinase. Cancer Res. 1997, 57, 4838-4848.

59. Johnson, J.R.; Cohen, M.; Sridhara, R.; Chen, Y.-F.; Williams, G.M.; Duan, J.; Gobburu, J.; Booth, B.; Benson, K.; Leighton, J.; et al. Approval Summary for Erlotinib for Treatment of Patients with Locally Advanced or Metastatic Non-Small Cell Lung Cancer after Failure of at Least One Prior Chemotherapy Regimen. Clin. Cancer Res. 2005, 11, 6414-6421. [CrossRef]

60. Shepherd, F.A.; Pereira, J.R.; Ciuleanu, T.; Tan, E.H.; Hirsh, V.; Thongprasert, S.; Campos, D.; Maoleekoonpiroj, S.; Smylie, M.; Martins, R.; et al. Erlotinib in Previously Treated Non-Small-Cell Lung Cancer. N. Engl. J. Med. 2005, 353, 123-132. [CrossRef] [PubMed]

61. Rusnak, D.W.; Lackey, K.; Affleck, K.; Wood, E.R.; Alligood, K.J.; Rhodes, N.; Keith, B.R.; Murray, D.M.; Knight, W.B.; Mullin, R.J.; et al. The Effects of the Novel, Reversible Epidermal Growth Factor Receptor/ErbB-2 Tyrosine Kinase Inhibitor, GW2016, on the Growth of Human Normal and Tumor-Derived Cell Lines in Vitro and in Vivo. Mol. Cancer 2001, 1, 85-94.

62. Higa, G.M.; Abraham, J. Lapatinib in the Treatment of Breast Cancer. Expert Rev. Anticancer Ther. 2014, 7, 1183-1192. [CrossRef] [PubMed]

63. Wood, E.R.; Truesdale, A.T.; McDonald, O.B.; Yuan, D.; Hassell, A.; Dickerson, S.H.; Ellis, B.; Pennisi, C.; Horne, E.; Lackey, K.; et al. A Unique Structure for Epidermal Growth Factor Receptor Bound to GW572016 (Lapatinib) Relationships among Protein Conformation, Inhibitor Off-Rate, and Receptor Activity in Tumor Cells. Cancer Res. 2004, 64, 6652-6659. [CrossRef] 
64. Shi, Y.; Zhang, L.; Liu, X.; Zhou, C.; Zhang, L.; Zhang, S.; Wang, D.; Li, Q.; Qin, S.; Hu, C.; et al. Icotinib versus Gefitinib in Previously Treated Advanced Non-Small-Cell Lung Cancer (ICOGEN): A Randomised, Double-Blind Phase 3 Non-Inferiority Trial. Lancet Oncol. 2013, 14, 953-961. [CrossRef]

65. Shi, Y.; Sun, Y.; Ding, C.; Wang, Z.; Wang, C.; Wang, Z.; Bai, C.; Bai, C.; Feng, J.; Liu, X.; et al. China Experts Consensus on Icotinib for Non-Small Cell Lung Cancer Treatment (2015 Version). J. Thorac. Dis 2015, 7, E468-E472. [CrossRef]

66. Liao, B.-C.; Lin, C.-C.; Yang, J.C.-H. Second and Third-Generation Epidermal Growth Factor Receptor Tyrosine Kinase Inhibitors in Advanced Nonsmall Cell Lung Cancer. Curr. Opin. Oncol. 2015, 27, 94-101. [CrossRef] [PubMed]

67. Solca, F.; Dahl, G.; Zoephel, A.; Bader, G.; Sanderson, M.; Klein, C.; Kraemer, O.; Himmelsbach, F.; Haaksma, E.; Adolf, G.R. Target Binding Properties and Cellular Activity of Afatinib (BIBW 2992), an Irreversible ErbB Family Blocker. J. Pharm. Exp. 2012, 343, 342-350. [CrossRef] [PubMed]

68. Sequist, L.V.; Yang, J.C.-H.; Yamamoto, N.; O’Byrne, K.; Hirsh, V.; Mok, T.; Geater, S.L.; Orlov, S.; Tsai, C.-M.; Boyer, M.; et al. Phase III Study of Afatinib or Cisplatin Plus Pemetrexed in Patients with Metastatic Lung Adenocarcinoma with EGFR Mutations. J. Clin. Oncol. 2013, 31, 3327-3334. [CrossRef]

69. Wu, Y.-L.; Zhou, C.; Hu, C.-P.; Feng, J.; Lu, S.; Huang, Y.; Li, W.; Hou, M.; Shi, J.H.; Lee, K.Y.; et al. Afatinib versus Cisplatin plus Gemcitabine for First-Line Treatment of Asian Patients with Advanced Non-Small-Cell Lung Cancer Harbouring EGFR Mutations (LUX-Lung 6): An Open-Label, Randomised Phase 3 Trial. Lancet Oncol. 2014, 15, 213-222. [CrossRef]

70. Rabindran, S.K.; Discafani, C.M.; Rosfjord, E.C.; Baxter, M.; Floyd, M.B.; Golas, J.; Hallett, W.A.; Johnson, B.D.; Nilakantan, R.; Overbeek, E.; et al. Antitumor Activity of HKI-272, an Orally Active, Irreversible Inhibitor of the HER-2 Tyrosine Kinase. Cancer Res. 2004, 64, 3958-3965. [CrossRef]

71. Dhillon, S. Neratinib in Early-Stage Breast Cancer: A Profile of Its Use in the EU. Clin. Drug Invest. 2019, 39, 221-229. [CrossRef]

72. Saura, C.; Oliveira, M.; Feng, Y.-H.; Dai, M.-S.; Chen, S.-W.; Hurvitz, S.A.; Kim, S.-B.; Moy, B.; Delaloge, S.; Gradishar, W.; et al. Neratinib Plus Capecitabine Versus Lapatinib Plus Capecitabine in HER2-Positive Metastatic Breast Cancer Previously Treated with $\geq 2$ HER2-Directed Regimens: Phase III NALA Trial. J. Clin. Oncol. 2020, 38, 3138-3149. [CrossRef] [PubMed]

73. Gonzales, A.J.; Hook, K.E.; Althaus, I.W.; Ellis, P.A.; Trachet, E.; Delaney, A.M.; Harvey, P.J.; Ellis, T.A.; Amato, D.M.; Nelson, J.M.; et al. Antitumor Activity and Pharmacokinetic Properties of PF-00299804, a Second-Generation Irreversible Pan-ErbB Receptor Tyrosine Kinase Inhibitor. Mol. Cancer 2008, 7, 1880-1889. [CrossRef] [PubMed]

74. Engelman, J.A.; Zejnullahu, K.; Gale, C.-M.; Lifshits, E.; Gonzales, A.J.; Shimamura, T.; Zhao, F.; Vincent, P.W.; Naumov, G.N.; Bradner, J.E.; et al. PF00299804, an Irreversible Pan-ERBB Inhibitor, Is Effective in Lung Cancer Models with EGFR and ERBB2 Mutations That Are Resistant to Gefitinib. Cancer Res. 2007, 67, 11924-11932. [CrossRef] [PubMed]

75. Lavacchi, D.; Mazzoni, F.; Giaccone, G. Clinical Evaluation of Dacomitinib for the Treatment of Metastatic Non-Small Cell Lung Cancer (NSCLC): Current Perspectives. Drug Des. Dev. 2019, 13, 3187-3198. [CrossRef] [PubMed]

76. Wu, L.; Ke, L.; Zhang, Z.; Yu, J.; Meng, X. Development of EGFR TKIs and Options to Manage Resistance of Third-Generation EGFR TKI Osimertinib: Conventional Ways and Immune Checkpoint Inhibitors. Front. Oncol. 2020, 10, 602762. [CrossRef] [PubMed]

77. Leonetti, A.; Sharma, S.; Minari, R.; Perego, P.; Giovannetti, E.; Tiseo, M. Resistance Mechanisms to Osimertinib in EGFR-Mutated Non-Small Cell Lung Cancer. Br. J. Cancer 2019, 121, 725-737. [CrossRef]

78. Kim, E.S. Olmutinib: First Global Approval. Drugs 2016, 76, 1153-1157. [CrossRef]

79. Yun, J.; Hong, M.H.; Kim, S.-Y.; Park, C.W.; Kim, S.-Y.; Yun, M.R.; Kang, H.N.; Pyo, K.H.; Lee, S.S.; Koh, J.S.; et al. YH25448, an Irreversible EGFR-TKI with Potent Intracranial Activity in EGFR Mutant Non-Small-Cell Lung Cancer. Clin. Cancer Res. 2019, 25, 2575-2587. [CrossRef]

80. Ahn, M.-J.; Han, J.-Y.; Lee, K.H.; Kim, S.-W.; Kim, D.-W.; Lee, Y.-G.; Cho, E.K.; Kim, J.-H.; Lee, G.-W.; Lee, J.-S.; et al. Lazertinib in Patients with EGFR Mutation-Positive Advanced Non-Small-Cell Lung Cancer: Results from the Dose Escalation and Dose Expansion Parts of a First-in-Human, Open-Label, Multicentre, Phase 1-2 Study. Lancet Oncol. 2019, 20, 1681-1690. [CrossRef]

81. Park, S.; Ku, B.M.; Jung, H.A.; Sun, J.-M.; Ahn, J.S.; Lee, S.-H.; Park, K.; Ahn, M.-J. EGFR C797S as a Resistance Mechanism of Lazertinib in Non-Small Cell Lung Cancer with EGFR T790M Mutation. Cancer Res. Treat. 2020, 52, 1288-1290. [CrossRef]

82. Piper-Vallillo, A.J.; Sequist, L.V.; Piotrowska, Z. Emerging Treatment Paradigms for EGFR-Mutant Lung Cancers Progressing on Osimertinib: A Review. J. Clin. Oncol. 2020, 38, 2926-2936. [CrossRef]

83. Jia, Y.; Yun, C.-H.; Park, E.; Ercan, D.; Manuia, M.; Juarez, J.; Xu, C.; Rhee, K.; Chen, T.; Zhang, H.; et al. Overcoming EGFR(T790M) and EGFR(C797S) Resistance with Mutant-Selective Allosteric Inhibitors. Nature 2016, 534, 129-132. [CrossRef]

84. Wang, S.; Song, Y.; Liu, D. EAI045: The Fourth-Generation EGFR Inhibitor Overcoming T790M and C797S Resistance. Cancer Lett. 2017, 385, 51-54. [CrossRef]

85. To, C.; Jang, J.; Chen, T.; Park, E.; Mushajiang, M.; Clercq, D.J.H.D.; Xu, M.; Wang, S.; Cameron, M.D.; Heppner, D.E.; et al. Single and Dual Targeting of Mutant EGFR with an Allosteric Inhibitor. Cancer Discov. 2019, 9, 926-943. [CrossRef]

86. Huang, W.-S.; Liu, S.; Zou, D.; Thomas, M.; Wang, Y.; Zhou, T.; Romero, J.; Kohlmann, A.; Li, F.; Qi, J.; et al. Discovery of Brigatinib (AP26113), a Phosphine Oxide-Containing, Potent, Orally Active Inhibitor of Anaplastic Lymphoma Kinase. J. Med. Chem. 2016, 59, 4948-4964. [CrossRef]

87. Uchibori, K.; Inase, N.; Araki, M.; Kamada, M.; Sato, S.; Okuno, Y.; Fujita, N.; Katayama, R. Brigatinib Combined with Anti-EGFR Antibody Overcomes Osimertinib Resistance in EGFR-Mutated Non-Small-Cell Lung Cancer. Nat. Commun. 2017, 8, 14768. [CrossRef] 
88. Wedge, S.R.; Ogilvie, D.J.; Dukes, M.; Kendrew, J.; Chester, R.; Jackson, J.A.; Boffey, S.J.; Valentine, P.J.; Curwen, J.O.; Musgrove, H.L.; et al. ZD6474 Inhibits Vascular Endothelial Growth Factor Signaling, Angiogenesis, and Tumor Growth Following Oral Administration. Cancer Res. 2002, 62, 4645-4655. [PubMed]

89. Morabito, A.; Piccirillo, M.C.; Falasconi, F.; Feo, G.D.; Giudice, A.D.; Bryce, J.; Maio, M.D.; Maio, E.D.; Normanno, N.; Perrone, F. Vandetanib (ZD6474), a Dual Inhibitor of Vascular Endothelial Growth Factor Receptor (VEGFR) and Epidermal Growth Factor Receptor (EGFR) Tyrosine Kinases: Current Status and Future Directions. Oncologist 2009, 14, 378-390. [CrossRef] [PubMed]

90. Cabanillas, M.E.; Ryder, M.; Jimenez, C. Targeted Therapy for Advanced Thyroid Cancer: Kinase Inhibitors and Beyond. Endocr. Rev. 2019, 40, 1573-1604. [CrossRef] [PubMed]

91. Hatem, R.; Labiod, D.; Château-Joubert, S.; de Plater, L.; Botty, R.E.; Vacher, S.; Bonin, F.; Servely, J.; Dieras, V.; Bièche, I.; et al. Vandetanib as a Potential New Treatment for Estrogen Receptor-negative Breast Cancers. Int. J. Cancer 2016, 138, $2510-2521$. [CrossRef]

92. Pedersen, M.W.; Pedersen, N.; Ottesen, L.H.; Poulsen, H.S. Differential Response to Gefitinib of Cells Expressing Normal EGFR and the Mutant EGFRvIII. Br. J. Cancer 2005, 93, 915-923. [CrossRef]

93. Tan, F.; Shen, X.; Wang, D.; Xie, G.; Zhang, X.; Ding, L.; Hu, Y.; He, W.; Wang, Y.; Wang, Y. Icotinib (BPI-2009H), a Novel EGFR Tyrosine Kinase Inhibitor, Displays Potent Efficacy in Preclinical Studies. Lung Cancer 2012, 76, 177-182. [CrossRef]

94. Li, D.; Ambrogio, L.; Shimamura, T.; Kubo, S.; Takahashi, M.; Chirieac, L.R.; Padera, R.F.; Shapiro, G.I.; Baum, A.; Himmelsbach, F.; et al. BIBW2992, an Irreversible EGFR/HER2 Inhibitor Highly Effective in Preclinical Lung Cancer Models. Oncogene 2008, 27, 4702-4711. [CrossRef]

95. Ferrarotto, R.; Gold, K.A. Afatinib in the Treatment of Head and Neck Squamous Cell Carcinoma. Expert Opin. Inv. Drug 2013, 23, 135-143. [CrossRef] [PubMed]

96. Hirano, T.; Yasuda, H.; Tani, T.; Hamamoto, J.; Oashi, A.; Ishioka, K.; Arai, D.; Nukaga, S.; Miyawaki, M.; Kawada, I.; et al. In Vitro Modeling to Determine Mutation Specificity of EGFR Tyrosine Kinase Inhibitors against Clinically Relevant EGFR Mutants in Non-Small-Cell Lung Cancer. Oncotarget 2015, 6, 38789-38803. [CrossRef] [PubMed]

97. Zhang, Q.; Zhang, L.; Yu, J.; Li, H.; He, S.; Tang, W.; Zuo, J.; Lu, W. Discovery of New BTK Inhibitors with B Cell Suppression Activity Bearing a 4,6-Substituted Thieno [3,2- d ]Pyrimidine Scaffold. Rsc Adv. 2017, 7, 26060-26069. [CrossRef]

98. Aldea, M.; Andre, F.; Marabelle, A.; Dogan, S.; Barlesi, F.; Soria, J.-C. Overcoming Resistance to Tumor-Targeted and ImmuneTargeted Therapies. Cancer Discov. 2021, 11, 874-899. [CrossRef] [PubMed]

99. McLaughlin, R.P.; He, J.; van der Noord, V.E.; Redel, J.; Foekens, J.A.; Martens, J.W.M.; Smid, M.; Zhang, Y.; van de Water, B. A Kinase Inhibitor Screen Identifies a Dual Cdc7/CDK9 Inhibitor to Sensitise Triple-Negative Breast Cancer to EGFR-Targeted Therapy. Breast Cancer Res. 2019, 21, 77. [CrossRef]

100. Sequist, L.V.; Waltman, B.A.; Dias-Santagata, D.; Digumarthy, S.; Turke, A.B.; Fidias, P.; Bergethon, K.; Shaw, A.T.; Gettinger, S.; Cosper, A.K.; et al. Genotypic and Histological Evolution of Lung Cancers Acquiring Resistance to EGFR Inhibitors. Sci. Transl. Med. 2011, 3, 75ra26. [CrossRef]

101. Chong, C.R.; Jänne, P.A. The Quest to Overcome Resistance to EGFR-Targeted Therapies in Cancer. Nat. Med. 2013, 19, 1389-1400. [CrossRef] [PubMed]

102. Nguyen, L.K.; Kholodenko, B.N. Feedback Regulation in Cell Signalling: Lessons for Cancer Therapeutics. Semin Cell Dev. Biol. 2016, 50, 85-94. [CrossRef] [PubMed]

103. Cremers, C.G.; Nguyen, L.K. Network Rewiring, Adaptive Resistance and Combating Strategies in Breast Cancer. Cancer Drug Resist. 2019. [CrossRef]

104. Pao, W.; Miller, V.A.; Politi, K.A.; Riely, G.J.; Somwar, R.; Zakowski, M.F.; Kris, M.G.; Varmus, H. Acquired Resistance of Lung Adenocarcinomas to Gefitinib or Erlotinib Is Associated with a Second Mutation in the EGFR Kinase Domain. PLoS Med. 2005, 2, e73. [CrossRef]

105. Kobayashi, S.; Boggon, T.J.; Dayaram, T.; Jänne, P.A.; Kocher, O.; Meyerson, M.; Johnson, B.E.; Eck, M.J.; Tenen, D.G.; Halmos, B. EGFR Mutation and Resistance of Non-Small-Cell Lung Cancer to Gefitinib. N. Engl. J. Med. 2005, 352, 786-792. [CrossRef]

106. Yun, C.-H.; Mengwasser, K.E.; Toms, A.V.; Woo, M.S.; Greulich, H.; Wong, K.-K.; Meyerson, M.; Eck, M.J. The T790M Mutation in EGFR Kinase Causes Drug Resistance by Increasing the Affinity for ATP. Proc. Natl. Acad. Sci. USA 2008, 105, $2070-2075$. [CrossRef]

107. Thress, K.S.; Paweletz, C.P.; Felip, E.; Cho, B.C.; Stetson, D.; Dougherty, B.; Lai, Z.; Markovets, A.; Vivancos, A.; Kuang, Y.; et al. Acquired EGFR C797S Mutation Mediates Resistance to AZD9291 in Non-Small Cell Lung Cancer Harboring EGFR T790M. Nat. Med. 2015, 21, 560-562. [CrossRef] [PubMed]

108. Oxnard, G.R.; Hu, Y.; Mileham, K.F.; Husain, H.; Costa, D.B.; Tracy, P.; Feeney, N.; Sholl, L.M.; Dahlberg, S.E.; Redig, A.J.; et al. Assessment of Resistance Mechanisms and Clinical Implications in Patients with EGFR T790M-Positive Lung Cancer and Acquired Resistance to Osimertinib. JAMA Oncol. 2018, 4, 1527. [CrossRef]

109. Song, H.-N.; Jung, K.S.; Yoo, K.H.; Cho, J.; Lee, J.Y.; Lim, S.H.; Kim, H.S.; Sun, J.-M.; Lee, S.-H.; Ahn, J.S.; et al. Acquired C797S Mutation upon Treatment with a T790M-Specific Third-Generation EGFR Inhibitor (HM61713) in Non-Small Cell Lung Cancer. J. Thorac. Oncol. 2016, 11, e45-e47. [CrossRef]

110. Chabon, J.J.; Simmons, A.D.; Lovejoy, A.F.; Esfahani, M.S.; Newman, A.M.; Haringsma, H.J.; Kurtz, D.M.; Stehr, H.; Scherer, F.; Karlovich, C.A.; et al. Circulating Tumour DNA Profiling Reveals Heterogeneity of EGFR Inhibitor Resistance Mechanisms in Lung Cancer Patients. Nat. Commun 2016, 7, 11815. [CrossRef] 
111. Tan, D.S.-W.; Kim, D.-W.; Leighl, N.B.; Riely, G.J.; Yang, J.C.-H.; Wolf, J.; Seto, T.; Felip, E.; Aix, S.P.; Jonnaert, M.; et al. Genomic Profiling of Resistant Tumor Samples Following Progression on EGF816, a Third Generation, Mutant-Selective EGFR Tyrosine Kinase Inhibitor (TKI), in Advanced Non-Small Cell Lung Cancer (NSCLC). J. Clin. Oncol. 2017, 35, 11506. [CrossRef]

112. Kim, A.; Jang, M.H.; Lee, S.J.; Bae, Y.K. Mutations of the Epidermal Growth Factor Receptor Gene in Triple-Negative Breast Cancer. J. Breast Cancer 2017, 20, 150-159. [CrossRef] [PubMed]

113. Teng, Y.H.-F.; Tan, W.-J.; Thike, A.-A.; Cheok, P.-Y.; Tse, G.M.-K.; Wong, N.-S.; Yip, G.W.-C.; Bay, B.-H.; Tan, P.-H. Mutations in the Epidermal Growth Factor Receptor (EGFR) Gene in Triple Negative Breast Cancer: Possible Implications for Targeted Therapy. Breast Cancer Res. 2011, 13, R35. [CrossRef] [PubMed]

114. Harrison, P.T.; Vyse, S.; Huang, P.H. Rare Epidermal Growth Factor Receptor (EGFR) Mutations in Non-Small Cell Lung Cancer. Semin. Cancer Biol. 2019, 61, 167-179. [CrossRef] [PubMed]

115. Santarpia, L.; Qi, Y.; Stemke-Hale, K.; Wang, B.; Young, E.J.; Booser, D.J.; Holmes, F.A.; O'Shaughnessy, J.; Hellerstedt, B.; Pippen, J.; et al. Mutation Profiling Identifies Numerous Rare Drug Targets and Distinct Mutation Patterns in Different Clinical Subtypes of Breast Cancers. Breast Cancer Res. Treat. 2012, 134, 333-343. [CrossRef]

116. Tilch, E.; Seidens, T.; Cocciardi, S.; Reid, L.E.; Byrne, D.; Simpson, P.T.; Vargas, A.C.; Cummings, M.C.; Fox, S.B.; Lakhani, S.R.; et al. Mutations in EGFR, BRAF and RAS Are Rare in Triple-Negative and Basal-like Breast Cancers from Caucasian Women. Breast Cancer Res. Treat. 2014, 143, 385-392. [CrossRef]

117. Reis-Filho, J.; Pinheiro, C.; Lambros, M.; Milanezi, F.; Carvalho, S.; Savage, K.; Simpson, P.; Jones, C.; Swift, S.; Mackay, A.; et al. EGFR Amplification and Lack of Activating Mutations in Metaplastic Breast Carcinomas. J. Pathol. 2006, 209, 445-453. [CrossRef]

118. Jacot, W.; Lopez-Crapez, E.; Thezenas, S.; Senal, R.; Fina, F.; Bibeau, F.; Romieu, G.; Lamy, P.-J. Lack of EGFR-Activating Mutations in European Patients with Triple-Negative Breast Cancer Could Emphasise Geographic and Ethnic Variations in Breast Cancer Mutation Profiles. Breast Cancer Res. 2011, 13, R133. [CrossRef]

119. Secq, V.; Villeret, J.; Fina, F.; Carmassi, M.; Carcopino, X.; Garcia, S.; Metellus, I.; Boubli, L.; Iovanna, J.; Charpin, C. Triple Negative Breast Carcinoma EGFR Amplification Is Not Associated with EGFR, Kras or ALK Mutations. Br. J. Cancer 2014, 110, 1045-1052. [CrossRef]

120. Grob, T.J.; Heilenkötter, U.; Geist, S.; Paluchowski, P.; Wilke, C.; Jaenicke, F.; Quaas, A.; Wilczak, W.; Choschzick, M.; Sauter, G.; et al. Rare Oncogenic Mutations of Predictive Markers for Targeted Therapy in Triple-Negative Breast Cancer. Breast Cancer Res. Treat. 2012, 134, 561-567. [CrossRef]

121. Kim, Y.; Kim, J.; Lee, H.-D.; Jeong, J.; Lee, W.; Lee, K.-A. Spectrum of EGFR Gene Copy Number Changes and KRAS Gene Mutation Status in Korean Triple Negative Breast Cancer Patients. PLoS ONE 2013, 8, e79014. [CrossRef]

122. Park, H.S.; Jang, M.H.; Kim, E.J.; Kim, H.J.; Lee, H.J.; Kim, Y.J.; Kim, J.H.; Kang, E.; Kim, S.-W.; Kim, I.A.; et al. High EGFR Gene Copy Number Predicts Poor Outcome in Triple-Negative Breast Cancer. Mod. Pathol. 2014, 27, 1212-1222. [CrossRef] [PubMed]

123. Lv, N.; Xie, X.; Ge, Q.; Lin, S.; Wang, X.; Kong, Y.; Shi, H.; Xie, X.; Wei, W. Epidermal Growth Factor Receptor in Breast Carcinoma: Association between Gene Copy Number and Mutations. Diagn. Pathol. 2011, 6, 118. [CrossRef] [PubMed]

124. Martin, V.; Botta, F.; Zanellato, E.; Molinari, F.; Crippa, S.; Mazzucchelli, L.; Frattini, M. Molecular Characterization of EGFR and EGFR-Downstream Pathways in Triple Negative Breast Carcinomas with Basal like Features. Histol. Histopathol. 2012, 27, 785-792. [PubMed]

125. Toyama, T.; Yamashita, H.; Kondo, N.; Okuda, K.; Takahashi, S.; Sasaki, H.; Sugiura, H.; Iwase, H.; Fujii, Y. Frequently Increased Epidermal Growth Factor Receptor (EGFR) Copy Numbers and Decreased BRCA1 MRNA Expression in Japanese Triple-Negative Breast Cancers. BMC Cancer 2008, 8, 309. [CrossRef] [PubMed]

126. Nakajima, H.; Ishikawa, Y.; Furuya, M.; Sano, T.; Ohno, Y.; Horiguchi, J.; Oyama, T. Protein Expression, Gene Amplification, and Mutational Analysis of EGFR in Triple-Negative Breast Cancer. Breast Cancer 2014, 21, 66-74. [CrossRef]

127. Lo, H.-W.; Hsu, S.-C.; Ali-Seyed, M.; Gunduz, M.; Xia, W.; Wei, Y.; Bartholomeusz, G.; Shih, J.-Y.; Hung, M.-C. Nuclear Interaction of EGFR and STAT3 in the Activation of the INOS/NO Pathway. Cancer Cell 2005, 7, 575-589. [CrossRef]

128. Lo, H.-W.; Hung, M.-C. Nuclear EGFR Signalling Network in Cancers: Linking EGFR Pathway to Cell Cycle Progression, Nitric Oxide Pathway and Patient Survival. Br. J. Cancer 2006, 94, 184-188. [CrossRef]

129. Huang, W.-C.; Chen, Y.-J.; Li, L.-Y.; Wei, Y.-L.; Hsu, S.-C.; Tsai, S.-L.; Chiu, P.-C.; Huang, W.-P.; Wang, Y.-N.; Chen, C.-H.; et al. Nuclear Translocation of Epidermal Growth Factor Receptor by Akt-Dependent Phosphorylation Enhances Breast CancerResistant Protein Expression in Gefitinib-Resistant Cells. J. Biol. Chem. 2011, 286, 20558-20568. [CrossRef] [PubMed]

130. Brand, T.M.; Iida, M.; Dunn, E.F.; Luthar, N.; Kostopoulos, K.T.; Corrigan, K.L.; Wleklinski, M.J.; Yang, D.; Wisinski, K.B.; Salgia, R.; et al. Nuclear Epidermal Growth Factor Receptor Is a Functional Molecular Target in Triple-Negative Breast Cancer. Mol. Cancer 2014, 13, 1356-1368. [CrossRef]

131. Lin, S.-Y.; Makino, K.; Xia, W.; Matin, A.; Wen, Y.; Kwong, K.Y.; Bourguignon, L.; Hung, M.-C. Nuclear Localization of EGF Receptor and Its Potential New Role as a Transcription Factor. Nat. Cell Biol. 2001, 3, 802-808. [CrossRef] [PubMed]

132. Hung, L.-Y.; Tseng, J.T.; Lee, Y.-C.; Xia, W.; Wang, Y.-N.; Wu, M.-L.; Chuang, Y.-H.; Lai, C.-H.; Chang, W.-C. Nuclear Epidermal Growth Factor Receptor (EGFR) Interacts with Signal Transducer and Activator of Transcription 5 (STAT5) in Activating Aurora-A Gene Expression. Nucleic Acids Res. 2008, 36, 4337-4351. [CrossRef] [PubMed]

133. Liccardi, G.; Hartley, J.A.; Hochhauser, D. EGFR Nuclear Translocation Modulates DNA Repair Following Cisplatin and Ionizing Radiation Treatment. Cancer Res. 2011, 71, 1103-1114. [CrossRef] [PubMed] 
134. Reddy, Y.V.R.; Ding, Q.; Lees-Miller, S.P.; Meek, K.; Ramsden, D.A. Non-Homologous End Joining Requires That the DNA-PK Complex Undergo an Autophosphorylation-Dependent Rearrangement at DNA Ends. J. Biol. Chem. 2004, $279,39408-39413$. [CrossRef]

135. Dittmann, K.; Mayer, C.; Rodemann, H.-P. Inhibition of Radiation-Induced EGFR Nuclear Import by C225 (Cetuximab) Suppresses DNA-PK Activity. Radiother. Oncol. 2005, 76, 157-161. [CrossRef]

136. Friedmann, B.J.; Caplin, M.; Savic, B.; Shah, T.; Lord, C.J.; Ashworth, A.; Hartley, J.A.; Hochhauser, D. Interaction of the Epidermal Growth Factor Receptor and the DNA-Dependent Protein Kinase Pathway Following Gefitinib Treatment. Mol. Cancer 2006, 5, 209-218. [CrossRef] [PubMed]

137. Ko, J.-C.; Ciou, S.-C.; Cheng, C.-M.; Wang, L.-H.; Hong, J.-H.; Jheng, M.-Y.; Ling, S.-T.; Lin, Y.-W. Involvement of Rad51 in Cytotoxicity Induced by Epidermal Growth Factor Receptor Inhibitor (Gefitinib, Iressa R) and Chemotherapeutic Agents in Human Lung Cancer Cells. Carcinogenesis 2008, 29, 1448-1458. [CrossRef]

138. Li, L.; Wang, H.; Yang, E.S.; Arteaga, C.L.; Xia, F. Erlotinib Attenuates Homologous Recombinational Repair of Chromosomal Breaks in Human Breast Cancer Cells. Cancer Res. 2008, 68, 9141-9146. [CrossRef]

139. Prat, M.; Oltolina, F.; Basilico, C. Monoclonal Antibodies against the MET/HGF Receptor and Its Ligand: Multitask Tools with Applications from Basic Research to Therapy. Biomedicines 2014, 2, 359-383. [CrossRef]

140. Deb, T.B.; Zuo, A.H.; Barndt, R.J.; Sengupta, S.; Jankovic, R.; Johnson, M.D. Pnck Overexpression in HER-2 Gene-Amplified Breast Cancer Causes Trastuzumab Resistance through a Paradoxical PTEN-Mediated Process. Breast Cancer Res. Treat. 2015, 150, 347-361. [CrossRef]

141. Riese, D.J., II. Ligand-Based Receptor Tyrosine Kinase Partial Agonists: New Paradigm for Cancer Drug Discovery? Expert Opin. Drug Dis. 2011, 6, 185-193. [CrossRef]

142. Gurdal, H.; Tuglu, M.M.; Bostanabad, S.Y.; Dalkiliç, B. Partial Agonistic Effect of Cetuximab on Epidermal Growth Factor Receptor and Src Kinase Activation in Triple-Negative Breast Cancer Cell Lines. Int. J. Oncol. 2019, 54, 1345-1356. [CrossRef]

143. Scott, G.K.; Dodson, J.M.; Montgomery, P.A.; Johnson, R.M.; Sarup, J.C.; Wong, W.L.; Ullrich, A.; Shepard, H.M.; Benz, C.C. P185HER2 Signal Transduction in Breast Cancer Cells. J. Biol. Chem. 1991, 266, 14300-14305. [CrossRef]

144. Nagata, Y.; Lan, K.-H.; Zhou, X.; Tan, M.; Esteva, F.J.; Sahin, A.A.; Klos, K.S.; Li, P.; Monia, B.P.; Nguyen, N.T.; et al. PTEN Activation Contributes to Tumor Inhibition by Trastuzumab, and Loss of PTEN Predicts Trastuzumab Resistance in Patients. Cancer Cell 2004, 6, 117-127. [CrossRef] [PubMed]

145. Yoshida, T.; Okamoto, I.; Okabe, T.; Iwasa, T.; Satoh, T.; Nishio, K.; Fukuoka, M.; Nakagawa, K. Matuzumab and Cetuximab Activate the Epidermal Growth Factor Receptor but Fail to Trigger Downstream Signaling by Akt or Erk. Int. J. Cancer 2008, 122, 1530-1538. [CrossRef] [PubMed]

146. Wagner, J.P.; Wolf-Yadlin, A.; Sevecka, M.; Grenier, J.K.; Root, D.E.; Lauffenburger, D.A.; MacBeath, G. Receptor Tyrosine Kinases Fall into Distinct Classes Based on Their Inferred Signaling Networks. Sci. Signal. 2013, 6, ra58. [CrossRef] [PubMed]

147. Wilson, T.R.; Fridlyand, J.; Yan, Y.; Penuel, E.; Burton, L.; Chan, E.; Peng, J.; Lin, E.; Wang, Y.; Sosman, J.; et al. Widespread Potential for Growth-Factor-Driven Resistance to Anticancer Kinase Inhibitors. Nature 2012, 487, 505-509. [CrossRef] [PubMed]

148. Wood, G.E.; Hockings, H.; Hilton, D.M.; Kermorgant, S. The Role of MET in Chemotherapy Resistance. Oncogene 2021, 40, 1927-1941. [CrossRef]

149. Jiao, Q.; Bi, L.; Ren, Y.; Song, S.; Wang, Q.; Wang, Y. Advances in Studies of Tyrosine Kinase Inhibitors and Their Acquired Resistance. Mol. Cancer 2018, 17, 36. [CrossRef] [PubMed]

150. Kim, Y.J.; Choi, J.; Seo, J.; Song, J.; Lee, S.E.; Kwon, M.J.; Kwon, M.J.; Kundu, J.; Jung, K.; Oh, E.; et al. MET Is a Potential Target for Use in Combination Therapy with EGFR Inhibition in Triple-negative/Basal-like Breast Cancer. Int. J. Cancer 2014, 134, $2424-2436$. [CrossRef]

151. Ponzo, M.G.; Lesurf, R.; Petkiewicz, S.; O’Malley, F.P.; Pinnaduwage, D.; Andrulis, I.L.; Bull, S.B.; Chughtai, N.; Zuo, D.; Souleimanova, M.; et al. Met Induces Mammary Tumors with Diverse Histologies and Is Associated with Poor Outcome and Human Basal Breast Cancer. Proc. Natl. Acad. Sci. USA 2009, 106, 12903-12908. [CrossRef]

152. Mueller, K.L.; Madden, J.M.; Zoratti, G.L.; Kuperwasser, C.; List, K.; Boerner, J.L. Fibroblast-Secreted Hepatocyte Growth Factor Mediates Epidermal Growth Factor Receptor Tyrosine Kinase Inhibitor Resistance in Triple-Negative Breast Cancers through Paracrine Activation of Met. Breast Cancer Res. 2012, 14, R104. [CrossRef]

153. Sohn, J.; Liu, S.; Parinyanitikul, N.; Lee, J.; Hortobagyi, G.N.; Mills, G.B.; Ueno, N.T.; Gonzalez-Angulo, A.M. CMET Activation and EGFR-Directed Therapy Resistance in Triple-Negative Breast Cancer. J. Cancer 2014, 5, 745-753. [CrossRef]

154. Kang, H.J.; Yi, Y.W.; Hong, Y.B.; Kim, H.J.; Jang, Y.-J.; Seong, Y.-S.; Bae, I. HER2 Confers Drug Resistance of Human Breast Cancer Cells through Activation of NRF2 by Direct Interaction. Sci. Rep. 2014, 4, 7201. [CrossRef] [PubMed]

155. Yi, Y.W.; Oh, S. Comparative Analysis of NRF2-Responsive Gene Expression in AcPC-1 Pancreatic Cancer Cell Line. Genes Genom. 2015, 37, 97-109. [CrossRef] [PubMed]

156. Jura, N.; Shan, Y.; Cao, X.; Shaw, D.E.; Kuriyan, J. Structural Analysis of the Catalytically Inactive Kinase Domain of the Human EGF Receptor 3. Proc. Natl. Acad. Sci. USA 2009, 106, 21608-21613. [CrossRef]

157. Sergina, N.V.; Rausch, M.; Wang, D.; Blair, J.; Hann, B.; Shokat, K.M.; Moasser, M.M. Escape from HER-Family Tyrosine Kinase Inhibitor Therapy by the Kinase-Inactive HER3. Nature 2007, 445, 437-441. [CrossRef] [PubMed]

158. Wheeler, D.L.; Huang, S.; Kruser, T.J.; Nechrebecki, M.M.; Armstrong, E.A.; Benavente, S.; Gondi, V.; Hsu, K.-T.; Harari, P.M. Mechanisms of Acquired Resistance to Cetuximab: Role of HER (ErbB) Family Members. Oncogene 2008, 27, 3944-3956. [CrossRef] 
159. Tao, J.J.; Castel, P.; Radosevic-Robin, N.; Elkabets, M.; Auricchio, N.; Aceto, N.; Weitsman, G.; Barber, P.; Vojnovic, B.; Ellis, H.; et al. Antagonism of EGFR and HER3 Enhances the Response to Inhibitors of the PI3K-Akt Pathway in Triple-Negative Breast Cancer. Sci. Signal. 2014, 7, ra29. [CrossRef]

160. Chakrabarty, A.; Sánchez, V.; Kuba, M.G.; Rinehart, C.; Arteaga, C.L. Feedback Upregulation of HER3 (ErbB3) Expression and Activity Attenuates Antitumor Effect of PI3K Inhibitors. Proc. Natl. Acad. Sci. USA 2012, 109, 2718-2723. [CrossRef] [PubMed]

161. Verma, N.; Müller, A.-K.; Kothari, C.; Panayotopoulou, E.; Kedan, A.; Selitrennik, M.; Mills, G.B.; Nguyen, L.K.; Shin, S.; Karn, T.; et al. Targeting of PYK2 Synergizes with EGFR Antagonists in Basal-like TNBC and Circumvents HER3-Associated Resistance via the NEDD4-NDRG1 Axis. Cancer Res. 2017, 77, 86-99. [CrossRef]

162. Ortiz-Cuaran, S.; Scheffler, M.; Plenker, D.; Dahmen, L.; Scheel, A.H.; Fernandez-Cuesta, L.; Meder, L.; Lovly, C.M.; Persigehl, T.; Merkelbach-Bruse, S.; et al. Heterogeneous Mechanisms of Primary and Acquired Resistance to Third-Generation EGFR Inhibitors. Clin. Cancer Res. 2016, 22, 4837-4847. [CrossRef] [PubMed]

163. Taniguchi, H.; Yamada, T.; Wang, R.; Tanimura, K.; Adachi, Y.; Nishiyama, A.; Tanimoto, A.; Takeuchi, S.; Araujo, L.H.; Boroni, M.; et al. AXL Confers Intrinsic Resistance to Osimertinib and Advances the Emergence of Tolerant Cells. Nat. Commun. 2019, 10, 259. [CrossRef]

164. Zhang, Z.; Lee, J.C.; Lin, L.; Olivas, V.; Au, V.; LaFramboise, T.; Abdel-Rahman, M.; Wang, X.; Levine, A.D.; Rho, J.K.; et al. Activation of the AXL Kinase Causes Resistance to EGFR-Targeted Therapy in Lung Cancer. Nat. Genet. 2012, 44, 852-860. [CrossRef] [PubMed]

165. Byers, L.A.; Diao, L.; Wang, J.; Saintigny, P.; Girard, L.; Peyton, M.; Shen, L.; Fan, Y.; Giri, U.; Tumula, P.K.; et al. An EpithelialMesenchymal Transition Gene Signature Predicts Resistance to EGFR and PI3K Inhibitors and Identifies Axl as a Therapeutic Target for Overcoming EGFR Inhibitor Resistance. Clin. Cancer Res. 2013, 19, 279-290. [CrossRef] [PubMed]

166. Meyer, A.S.; Miller, M.A.; Gertler, F.B.; Lauffenburger, D.A. The Receptor AXL Diversifies EGFR Signaling and Limits the Response to EGFR-Targeted Inhibitors in Triple-Negative Breast Cancer Cells. Sci. Signal. 2013, 6, ra66. [CrossRef]

167. Lev, S. Targeted Therapy and Drug Resistance in Triple-Negative Breast Cancer: The EGFR Axis. Biochem. Soc. Trans. 2020, 48, 657-665. [CrossRef] [PubMed]

168. Duncan, J.S.; Whittle, M.C.; Nakamura, K.; Abell, A.N.; Midland, A.A.; Zawistowski, J.S.; Johnson, N.L.; Granger, D.A.; Jordan, N.V.; Darr, D.B.; et al. Dynamic Reprogramming of the Kinome in Response to Targeted MEK Inhibition in Triple-Negative Breast Cancer. Cell 2012, 149, 307-321. [CrossRef]

169. Sears, R.; Nuckolls, F.; Haura, E.; Taya, Y.; Tamai, K.; Nevins, J.R. Multiple Ras-Dependent Phosphorylation Pathways Regulate Myc Protein Stability. Gene Dev. 2000, 14, 2501-2514. [CrossRef] [PubMed]

170. Yi, Y.W.; Kang, H.J.; Kim, H.J.; Hwang, J.S.; Wang, A.; Bae, I. Inhibition of Constitutively Activated Phosphoinositide 3kinase/AKT Pathway Enhances Antitumor Activity of Chemotherapeutic Agents in Breast Cancer Susceptibility Gene 1-defective Breast Cancer Cells. Mol. Carcinog. 2013, 52, 667-675. [CrossRef]

171. Yi, Y.W.; Kang, H.J.; Bae, E.J.; Oh, S.; Seong, Y.-S.; Bae, I. $\beta$-TrCP1 Degradation Is a Novel Action Mechanism of PI3K/MTOR Inhibitors in Triple-Negative Breast Cancer Cells. Exp. Mol. Med. 2015, 47, e143. [CrossRef] [PubMed]

172. Hou, S.; Yi, Y.W.; Kang, H.J.; Zhang, L.; Kim, H.J.; Kong, Y.; Liu, Y.; Wang, K.; Kong, H.-S.; Grindrod, S.; et al. Novel Carbazole Inhibits Phospho-STAT3 through Induction of Protein-Tyrosine Phosphatase PTPN6. J. Med. Chem. 2014, 57, 6342-6353. [CrossRef]

173. Kang, H.J.; Yi, Y.W.; Hou, S.-J.; Kim, H.J.; Kong, Y.; Bae, I.; Brown, M.L. Disruption of STAT3-DNMT1 Interaction by SH-I-14 Induces Re-Expression of Tumor Suppressor Genes and Inhibits Growth of Triple-Negative Breast Tumor. Oncotarget 2014, 5, 83457-83468. [CrossRef] [PubMed]

174. Schubbert, S.; Shannon, K.; Bollag, G. Hyperactive Ras in Developmental Disorders and Cancer. Nat. Rev. Cancer 2007, 7, 295-308. [CrossRef] [PubMed]

175. Hoeflich, K.P.; O’Brien, C.; Boyd, Z.; Cavet, G.; Guerrero, S.; Jung, K.; Januario, T.; Savage, H.; Punnoose, E.; Truong, T.; et al. In Vivo Antitumor Activity of MEK and Phosphatidylinositol 3-Kinase Inhibitors in Basal-Like Breast Cancer Models. Clin. Cancer Res. 2009, 15, 4649-4664. [CrossRef]

176. Giltnane, J.M.; Balko, J.M. Rationale for Targeting the Ras/MAPK Pathway in Triple-Negative Breast Cancer. Discov. Med. 2014, 17, 275-283. [PubMed]

177. Paranjape, T.; Heneghan, H.; Lindner, R.; Keane, F.K.; Hoffman, A.; Hollestelle, A.; Dorairaj, J.; Geyda, K.; Pelletier, C.; Nallur, S.; et al. A 3'-Untranslated Region KRAS Variant and Triple-Negative Breast Cancer: A Case-Control and Genetic Analysis. Lancet Oncol. 2011, 12, 377-386. [CrossRef]

178. Tokumaru, Y.; Oshi, M.; Katsuta, E.; Yan, L.; Satyananda, V.; Matsuhashi, N.; Futamura, M.; Akao, Y.; Yoshida, K.; Takabe, K. KRAS Signaling Enriched Triple Negative Breast Cancer Is Associated with Favorable Tumor Immune Microenvironment and Better Survival. Am. J. Cancer Res. 2020, 10, 897-907.

179. Popov, N.; Schülein, C.; Jaenicke, L.A.; Eilers, M. Ubiquitylation of the Amino Terminus of Myc by SCF $\beta$-TrCP Antagonizes SCFFbw7-Mediated Turnover. Nat. Cell Biol. 2010, 12, 973-981. [CrossRef]

180. Horiuchi, D.; Camarda, R.; Zhou, A.Y.; Yau, C.; Momcilovic, O.; Balakrishnan, S.; Corella, A.N.; Eyob, H.; Kessenbrock, K.; Lawson, D.A.; et al. PIM1 Kinase Inhibition as a Targeted Therapy against Triple-Negative Breast Tumors with Elevated MYC Expression. Nat. Med. 2016, 22, 1321-1329. [CrossRef] 
181. Lee, K.; Giltnane, J.M.; Balko, J.M.; Schwarz, L.J.; Guerrero-Zotano, A.L.; Hutchinson, K.E.; Nixon, M.J.; Estrada, M.V.; Sánchez, V.; Sanders, M.E.; et al. MYC and MCL1 Cooperatively Promote Chemotherapy-Resistant Breast Cancer Stem Cells via Regulation of Mitochondrial Oxidative Phosphorylation. Cell Metab. 2017, 26, 633-647.e7. [CrossRef] [PubMed]

182. Carey, J.P.; Karakas, C.; Bui, T.; Chen, X.; Vijayaraghavan, S.; Zhao, Y.; Wang, J.; Mikule, K.; Litton, J.K.; Hunt, K.K.; et al. Synthetic Lethality of PARP Inhibitors in Combination with MYC Blockade Is Independent of BRCA Status in Triple Negative Breast Cancer. Cancer Res. 2017, 78, 742-757. [CrossRef]

183. Kipreos, E.T.; Pagano, M. The F-Box Protein Family. Genome Biol. 2000, 1. [CrossRef]

184. Wang, Z.; Liu, P.; Inuzuka, H.; Wei, W. Roles of F-Box Proteins in Cancer. Nat. Rev. Cancer 2014, 14, 233-247. [CrossRef] [PubMed]

185. Lau, A.W.; Liu, Y.; Tron, A.E.; Inuzuka, H.; Wei, W. SCF and APC E3 Ubiquitin Ligases in Tumorigenesis; Springer Briefs in Cancer Research 2014; Springer: Berlin/Heidelberg, Germany, 2014; pp. 15-45. [CrossRef]

186. Duan, S.; Skaar, J.R.; Kuchay, S.; Toschi, A.; Kanarek, N.; Ben-Neriah, Y.; Pagano, M. MTOR Generates an Auto-Amplification Loop by Triggering the BTrCP- and CK1 $\alpha$-Dependent Degradation of DEPTOR. Mol. Cell 2011, 44, 317-324. [CrossRef]

187. Gao, D.; Inuzuka, H.; Tan, M.-K.M.; Fukushima, H.; Locasale, J.W.; Liu, P.; Wan, L.; Zhai, B.; Chin, Y.R.; Shaik, S.; et al. MTOR Drives Its Own Activation via SCF $\beta$ TrCP-Dependent Degradation of the MTOR Inhibitor DEPTOR. Mol. Cell 2011, 44, 290-303. [CrossRef] [PubMed]

188. Zhao, Y.; Xiong, X.; Sun, Y. DEPTOR, an MTOR Inhibitor, Is a Physiological Substrate of SCF $\beta$ TrCP E3 Ubiquitin Ligase and Regulates Survival and Autophagy. Mol. Cell 2011, 44, 304-316. [CrossRef]

189. Karakas, B.; Bachman, K.E.; Park, B.H. Mutation of the PIK3CA Oncogene in Human Cancers. Br. J. Cancer 2006, 94, 455-459. [CrossRef]

190. Shah, S.P.; Roth, A.; Goya, R.; Oloumi, A.; Ha, G.; Zhao, Y.; Turashvili, G.; Ding, J.; Tse, K.; Haffari, G.; et al. The Clonal and Mutational Evolution Spectrum of Primary Triple-Negative Breast Cancers. Nature 2012, 486, 395-399. [CrossRef]

191. Cossu-Rocca, P.; Orrù, S.; Muroni, M.R.; Sanges, F.; Sotgiu, G.; Ena, S.; Pira, G.; Murgia, L.; Manca, A.; Uras, M.G.; et al. Analysis of PIK3CA Mutations and Activation Pathways in Triple Negative Breast Cancer. PLoS ONE 2015, 10, e0141763. [CrossRef]

192. Koboldt, D.C.; Fulton, R.S.; McLellan, M.D.; Schmidt, H.; Kalicki-Veizer, J.; McMichael, J.F.; Fulton, L.L.; Dooling, D.J.; Ding, L.; Mardis, E.R.; et al. Comprehensive Molecular Portraits of Human Breast Tumours. Nature 2012, 490, 61-70. [CrossRef]

193. Maehama, T.; Dixon, J.E. The Tumor Suppressor, PTEN/MMAC1, Dephosphorylates the Lipid Second Messenger, Phosphatidylinositol 3,4,5-Trisphosphate. J. Biol. Chem. 1998, 273, 13375-13378. [CrossRef]

194. Chen, C.-Y.; Chen, J.; He, L.; Stiles, B.L. PTEN: Tumor Suppressor and Metabolic Regulator. Front. Endocrinol. 2018, 9, 338. [CrossRef]

195. Hu, H.; Zhu, J.; Zhong, Y.; Geng, R.; Ji, Y.; Guan, Q.; Hong, C.; Wei, Y.; Min, N.; Qi, A.; et al. PIK3CA Mutation Confers Resistance to Chemotherapy in Triple-Negative Breast Cancer by Inhibiting Apoptosis and Activating the PI3K/AKT/MTOR Signaling Pathway. Ann. Transl. Med. 2021, 9, 410. [CrossRef]

196. Young, C.D.; Zimmerman, L.J.; Hoshino, D.; Formisano, L.; Hanker, A.B.; Gatza, M.L.; Morrison, M.M.; Moore, P.D.; Whitwell, C.A.; Dave, B.; et al. Activating PIK3CA Mutations Induce an Epidermal Growth Factor Receptor (EGFR)/Extracellular SignalRegulated Kinase (ERK) Paracrine Signaling Axis in Basal-like Breast Cancer. Mol. Cell Proteom. 2015, 14, 1959-1976. [CrossRef] [PubMed]

197. Chin, Y.R.; Yoshida, T.; Marusyk, A.; Beck, A.H.; Polyak, K.; Toker, A. Targeting Akt3 Signaling in Triple-Negative Breast Cancer. Cancer Res. 2014, 74, 964-973. [CrossRef]

198. Fan, Y.; Dutta, J.; Gupta, N.; Fan, G.; Gélinas, C. Regulation of Programmed Cell Death by NF-kB and its Role in Tumorigenesis and Therapy. In Programmed Cell Death in Cancer Progression and Therapy; Khosravi-Far, R., White, E., Eds.; Springer: Berlin/Heidelberg, Germany, 2008; pp. 223-250. ISBN 9781402065538.

199. Chaturvedi, M.M.; Sung, B.; Yadav, V.R.; Kannappan, R.; Aggarwal, B.B. NF-KB Addiction and Its Role in Cancer: 'One Size Does Not Fit All'. Oncogene 2011, 30, 1615-1630. [CrossRef] [PubMed]

200. Karin, M.; Cao, Y.; Greten, F.R.; Li, Z.-W. NF-KB in Cancer: From Innocent Bystander to Major Culprit. Nat. Rev. Cancer 2002, 2, 301-310. [CrossRef] [PubMed]

201. Karin, M. The IкB Kinase-a Bridge between Inflammation and Cancer. Cell Res. 2008, 18, 334-342. [CrossRef]

202. De, S.; Dermawan, J.K.T.; Stark, G.R. EGF Receptor Uses SOS1 to Drive Constitutive Activation of NFkB in Cancer Cells. Proc. Natl. Acad. Sci. USA 2014, 111, 11721-11726. [CrossRef]

203. Sethi, G.; Ahn, K.S.; Chaturvedi, M.M.; Aggarwal, B.B. Epidermal Growth Factor (EGF) Activates Nuclear Factor-KB through IkB $\alpha$ Kinase-Independent but EGF Receptor-Kinase Dependent Tyrosine 42 Phosphorylation of IkB $\alpha$. Oncogene 2007, 26, 7324-7332. [CrossRef] [PubMed]

204. Häussler, U.; von Wichert, G.; Schmid, R.M.; Keller, F.; Schneider, G. Epidermal Growth Factor Activates Nuclear Factor-KB in Human Proximal Tubule Cells. Am. J. Physiol. Ren. 2005, 289, F808-F815. [CrossRef] [PubMed]

205. Alberti, C.; Pinciroli, P.; Valeri, B.; Ferri, R.; Ditto, A.; Umezawa, K.; Sensi, M.; Canevari, S.; Tomassetti, A. Ligand-Dependent EGFR Activation Induces the Co-Expression of IL-6 and PAI-1 via the NFkB Pathway in Advanced-Stage Epithelial Ovarian Cancer. Oncogene 2012, 31, 4139-4149. [CrossRef]

206. Sun, L.; Carpenter, G. Epidermal Growth Factor Activation of NF-KB Is Mediated through IkB $\alpha$ Degradation and Intracellular Free Calcium. Oncogene 1998, 16, 2095-2102. [CrossRef] [PubMed] 
207. Matsumoto, A.; Deyama, Y.; Deyama, A.; Okitsu, M.; Yoshimura, Y.; Suzuki, K. Epidermal Growth Factor Receptor-Mediated Expression of NF-KB Transcription Factor in Osteoblastic MC3T3-E1 Cells Cultured under a Low-Calcium Environment. Life Sci. 1998, 62, 1623-1627. [CrossRef]

208. Obata, H.; Biro, S.; Arima, N.; Kaieda, H.; Kihara, T.; Eto, H.; Miyata, M.; Tanaka, H. NF-KB Is Induced in the Nuclei of Cultured Rat Aortic Smooth Muscle Cells by Stimulation of Various Growth Factors. Biochem. Bioph Res. Commun. 1996, $224,27-32$. [CrossRef]

209. Biswas, D.K.; Cruz, A.P.; Gansberger, E.; Pardee, A.B. Epidermal Growth Factor-Induced Nuclear Factor KB Activation: A Major Pathway of Cell-Cycle Progression in Estrogen-Receptor Negative Breast Cancer Cells. Proc. Natl. Acad. Sci. USA 2000, 97, 8542-8547. [CrossRef]

210. Hardbower, D.M.; Singh, K.; Asim, M.; Verriere, T.G.; Olivares-Villagómez, D.; Barry, D.P.; Allaman, M.M.; Washington, M.K.; Peek, R.M.; Piazuelo, M.B.; et al. EGFR Regulates Macrophage Activation and Function in Bacterial Infection. J. Clin. Invest. 2016, 126, 3296-3312. [CrossRef]

211. Shostak, K.; Zhang, X.; Hubert, P.; Göktuna, S.I.; Jiang, Z.; Klevernic, I.; Hildebrand, J.; Roncarati, P.; Hennuy, B.; Ladang, A.; et al. NF-KB-Induced KIAA1199 Promotes Survival through EGFR Signalling. Nat. Commun. 2014, 5, 5232. [CrossRef]

212. Nottingham, L.K.; Yan, C.H.; Yang, X.; Si, H.; Coupar, J.; Bian, Y.; Cheng, T.-F.; Allen, C.; Arun, P.; Gius, D.; et al. Aberrant IKK $\alpha$ and IKK $\beta$ Cooperatively Activate NF-KB and Induce EGFR/AP1 Signaling to Promote Survival and Migration of Head and Neck Cancer. Oncogene 2014, 33, 1135-1147. [CrossRef]

213. Kung, C.-P.; Raab-Traub, N. Epstein-Barr Virus Latent Membrane Protein 1 Modulates Distinctive NF-KB Pathways through C-Terminus-Activating Region 1 To Regulate Epidermal Growth Factor Receptor Expression $\nabla$. J. Virol. 2010, 84, 6605-6614. [CrossRef]

214. Li, Z.; Yang, Z.; Passaniti, A.; Lapidus, R.G.; Liu, X.; Cullen, K.J.; Dan, H.C. A Positive Feedback Loop Involving EGFR/Akt/MTORC1 and IKK/NF-KB Regulates Head and Neck Squamous Cell Carcinoma Proliferation. Oncotarget 2015, 7, 31892-31906. [CrossRef]

215. Makhov, P.; Naito, S.; Haifler, M.; Kutikov, A.; Boumber, Y.; Uzzo, R.G.; Kolenko, V.M. The Convergent Roles of NF-KB and ER Stress in Sunitinib-Mediated Expression of pro-Tumorigenic Cytokines and Refractory Phenotype in Renal Cell Carcinoma. Cell Death Dis. 2018, 9, 374. [CrossRef] [PubMed]

216. Day, E.K.; Sosale, N.G.; Xiao, A.; Zhong, Q.; Purow, B.; Lazzara, M.J. Glioblastoma Cell Resistance to EGFR and MET Inhibition Can Be Overcome via Blockade of FGFR-SPRY2 Bypass Signaling. Cell Rep. 2020, 30, 3383-3396.e7. [CrossRef] [PubMed]

217. Bivona, T.G.; Hieronymus, H.; Parker, J.; Chang, K.; Taron, M.; Rosell, R.; Moonsamy, P.; Dahlman, K.; Miller, V.A.; Costa, C.; et al. FAS and NF-KB Signalling Modulate Dependence of Lung Cancers on Mutant EGFR. Nature 2011, 471, 523-526. [CrossRef]

218. Tian, R.; Li, Y.; Gao, M. Shikonin Causes Cell-Cycle Arrest and Induces Apoptosis by Regulating the EGFR-NF-KB Signalling Pathway in Human Epidermoid Carcinoma A431 Cells. Biosci. Rep. 2015, 35, e00189. [CrossRef]

219. Galvani, E.; Sun, J.; Leon, L.G.; Sciarrillo, R.; Narayan, R.S.; Sjin, R.T.T.; Lee, K.; Ohashi, K.; Heideman, D.A.M.; Alfieri, R.R.; et al NF-KB Drives Acquired Resistance to a Novel Mutant-Selective EGFR Inhibitor. Oncotarget 2015, 6, 42717-42732. [CrossRef] [PubMed]

220. Hiraki, M.; Maeda, T.; Mehrotra, N.; Jin, C.; Alam, M.; Bouillez, A.; Hata, T.; Tagde, A.; Keating, A.; Kharbanda, S.; et al. Targeting MUC1-C Suppresses BCL2A1 in Triple-Negative Breast Cancer. Signal. Transduct. Target. 2018, 3, 13. [CrossRef]

221. Ahmad, R.; Raina, D.; Joshi, M.D.; Kawano, T.; Ren, J.; Kharbanda, S.; Kufe, D. MUC1-C Oncoprotein Functions as a Direct Activator of the Nuclear Factor-KB P65 Transcription Factor. Cancer Res. 2009, 69, 7013-7021. [CrossRef] [PubMed]

222. Kufe, D.W. MUC1-C Oncoprotein as a Target in Breast Cancer: Activation of Signaling Pathways and Therapeutic Approaches. Oncogene 2013, 32, 1073-1081. [CrossRef] [PubMed]

223. Siroy, A.; Abdul-Karim, F.W.; Miedler, J.; Fong, N.; Fu, P.; Gilmore, H.; Baar, J. MUC1 Is Expressed at High Frequency in Early-Stage Basal-like Triple-Negative Breast Cancer. Hum. Pathol. 2013, 44, 2159-2166. [CrossRef] [PubMed]

224. Llona-Minguez, S.; Baiget, J.; Mackay, S.P. Small-Molecule Inhibitors of IкB Kinase (IKK) and IKK-Related Kinases. Pharm Pat. Anal. 2013, 2, 481-498. [CrossRef] [PubMed]

225. Wang, X.; Chao, L.; Li, X.; Ma, G.; Chen, L.; Zang, Y.; Zhou, G. Elevated Expression of Phosphorylated C-Jun NH2-Terminal Kinase in Basal-like and "Triple-Negative" Breast Cancers. Hum. Pathol 2010, 41, 401-406. [CrossRef] [PubMed]

226. Xie, X.; Kaoud, T.S.; Edupuganti, R.; Zhang, T.; Kogawa, T.; Zhao, Y.; Chauhan, G.B.; Giannoukos, D.N.; Qi, Y.; Tripathy, D.; et al. C-Jun N-Terminal Kinase Promotes Stem Cell Phenotype in Triple-Negative Breast Cancer through Upregulation of Notch1 via Activation of c-Jun. Oncogene 2017, 36, 2599-2608. [CrossRef] [PubMed]

227. Gee, J.M.W.; Barroso, A.F.; Ellis, I.O.; Robertson, J.F.R.; Nicholson, R.I. Biological and Clinical Associations of C-jun Activation in Human Breast Cancer. Int. J. Cancer 2000, 89, 177-186. [CrossRef]

228. Vleugel, M.M.; Greijer, A.E.; Bos, R.; van der Wall, E.; van Diest, P.J. C-Jun Activation Is Associated with Proliferation and Angiogenesis in Invasive Breast Cancer. Hum. Pathol. 2006, 37, 668-674. [CrossRef]

229. Manole, S.; Richards, E.J.; Meyer, A.S. JNK Pathway Activation Modulates Acquired Resistance to EGFR/HER2-Targeted Therapies. Cancer Res. 2016, 76, 5219-5228. [CrossRef]

230. Han, J.; Jeon, M.; Shin, I.; Kim, S. Elevated STC-1 Augments the Invasiveness of Triple-Negative Breast Cancer Cells through Activation of the JNK/c-Jun Signaling Pathway. Oncol. Rep. 2016, 36, 1764-1771. [CrossRef] 
231. Ebelt, N.D.; Kaoud, T.S.; Edupuganti, R.; Ravenstein, S.V.; Dalby, K.N.; Van, C.L. A C-Jun N-Terminal Kinase Inhibitor, JNK-IN-8, Sensitizes Triple Negative Breast Cancer Cells to Lapatinib. Oncotarget 2017, 5, 104894-104912. [CrossRef]

232. Giuli, M.V.; Giuliani, E.; Screpanti, I.; Bellavia, D.; Checquolo, S. Notch Signaling Activation as a Hallmark for Triple-Negative Breast Cancer Subtype. J. Oncol. 2019, 2019, 1-15. [CrossRef]

233. BeLow, M.; Osipo, C. Notch Signaling in Breast Cancer: A Role in Drug Resistance. Cells 2020, 9, 2204. [CrossRef]

234. Diluvio, G.; Gaudio, F.D.; Giuli, M.V.; Franciosa, G.; Giuliani, E.; Palermo, R.; Besharat, Z.M.; Pignataro, M.G.; Vacca, A.; d'Amati, G.; et al. NOTCH3 Inactivation Increases Triple Negative Breast Cancer Sensitivity to Gefitinib by Promoting EGFR Tyrosine Dephosphorylation and Its Intracellular Arrest. Oncogenesis 2018, 7, 42. [CrossRef]

235. Oren, M.; Rotter, V. Mutant P53 Gain-of-Function in Cancer. Cold Spring Harb. Perspect. Biol. 2010, 2, a001107. [CrossRef]

236. Maslon, M.M.; Hupp, T.R. Drug Discovery and Mutant P53. Trends Cell Biol. 2010, 20, 542-555. [CrossRef]

237. Goh, A.M.; Coffill, C.R.; Lane, D.P. The Role of Mutant P53 in Human Cancer. J. Pathol. 2011, 223, 116-126. [CrossRef] [PubMed]

238. Muller, P.A.J.; Vousden, K.H.; Norman, J.C. P53 and Its Mutants in Tumor Cell Migration and Invasion. J. Cell Biol. 2011, 192, 209-218. [CrossRef] [PubMed]

239. Alvarado-Ortiz, E.; de la Cruz-López, K.G.; Becerril-Rico, J.; Sarabia-Sánchez, M.A.; Ortiz-Sánchez, E.; García-Carrancá, A. Mutant P53 Gain-of-Function: Role in Cancer Development, Progression, and Therapeutic Approaches. Front. Cell Dev. Biol. 2021, 8, 607670. [CrossRef] [PubMed]

240. Yi, Y.W.; Kang, H.J.; Kim, H.J.; Kong, Y.; Brown, M.M.; Bae, I. Targeting Mutant P53 by a SIRT1 Activator YK-3-237 Inhibits the Proliferation of Triple-Negative Breast Cancer Cells. Oncotarget 2013, 4, 984-994. [CrossRef] [PubMed]

241. Darb-Esfahani, S.; Denkert, C.; Stenzinger, A.; Salat, C.; Sinn, B.; Schem, C.; Endris, V.; Klare, P.; Schmitt, W.; Blohmer, J.-U.; et al. Role of TP53 Mutations in Triple Negative and HER2-Positive Breast Cancer Treated with Neoadjuvant Anthracycline/TaxaneBased Chemotherapy. Oncotarget 2016, 7, 67686-67698. [CrossRef]

242. Hientz, K.; Mohr, A.; Bhakta-Guha, D.; Efferth, T. The Role of P53 in Cancer Drug Resistance and Targeted Chemotherapy. Oncotarget 2017, 8, 8921-8946. [CrossRef]

243. Coradini, D.; Biganzoli, E.; Ardoino, I.; Ambrogi, F.; Boracchi, P.; Demicheli, R.; Daidone, M.G.; Moliterni, A. P53 Status Identifies Triple-Negative Breast Cancer Patients Who Do Not Respond to Adjuvant Chemotherapy. Breast 2015, 24, 294-297. [CrossRef]

244. Shapira, I.; Lee, A.; Vora, R.; Budman, D.R. P53 Mutations in Triple Negative Breast Cancer Upregulate Endosomal Recycling of Epidermal Growth Factor Receptor (EGFR) Increasing Its Oncogenic Potency. Crit Rev. Oncol. Hematol. 2013, 88, 284-292. [CrossRef]

245. Neilsen, P.M.; Noll, J.E.; Mattiske, S.; Bracken, C.P.; Gregory, P.A.; Schulz, R.B.; Lim, S.P.; Kumar, R.; Suetani, R.J.; Goodall, G.J.; et al. Mutant P53 Drives Invasion in Breast Tumors through Up-Regulation of MiR-155. Oncogene 2013, 32, 2992-3000. [CrossRef] [PubMed]

246. Wang, W.; Cheng, B.; Miao, L.; Mei, Y.; Wu, M. Mutant P53-R273H Gains New Function in Sustained Activation of EGFR Signaling via Suppressing MiR-27a Expression. Cell Death Dis. 2013, 4, e574. [CrossRef]

247. Steinman, R.M.; Mellman, I.S.; Muller, W.A.; Cohn, Z.A. Endocytosis and the Recycling of Plasma Membrane. J. Cell Biol. 1983, 96, 1-27. [CrossRef] [PubMed]

248. Yi, Y.W.; Lee, J.H.; Kim, S.-Y.; Pack, C.-G.; Ha, D.H.; Park, S.R.; Youn, J.; Cho, B.S. Advances in Analysis of Biodistribution of Exosomes by Molecular Imaging. Int. J. Mol. Sci. 2020, 21, 665. [CrossRef]

249. Ha, D.H.; Kim, H.; Lee, J.; Kwon, H.H.; Park, G.-H.; Yang, S.H.; Jung, J.Y.; Choi, H.; Lee, J.H.; Sung, S.; et al. Mesenchymal Stem/Stromal Cell-Derived Exosomes for Immunomodulatory Therapeutics and Skin Regeneration. Cells 2020, 9, 1157. [CrossRef] [PubMed]

250. Grant, B.D.; Donaldson, J.G. Pathways and Mechanisms of Endocytic Recycling. Nat. Rev. Mol. Cell Bio 2009, 10, 597-608. [CrossRef]

251. Muller, P.A.J.; Caswell, P.T.; Doyle, B.; Iwanicki, M.P.; Tan, E.H.; Karim, S.; Lukashchuk, N.; Gillespie, D.A.; Ludwig, R.L.; Gosselin, P.; et al. Mutant P53 Drives Invasion by Promoting Integrin Recycling. Cell 2009, 139, 1327-1341. [CrossRef] [PubMed]

252. Caswell, P.T.; Chan, M.; Lindsay, A.J.; McCaffrey, M.W.; Boettiger, D.; Norman, J.C. Rab-Coupling Protein Coordinates Recycling of A5 31 Integrin and EGFR1 to Promote Cell Migration in 3D Microenvironments. J. Cell Biol. 2008, 183, 143-155. [CrossRef]

253. Shahar, N.; Larisch, S. Inhibiting the Inhibitors: Targeting Anti-Apoptotic Proteins in Cancer and Therapy Resistance. Drug Resist. Updates 2020, 52, 100712. [CrossRef]

254. Giménez-Bonafé, P.; Tortosa, A.; Pérez-Tomás, R. Overcoming Drug Resistance by Enhancing Apoptosis of Tumor Cells. Curr. Cancer Drug Targets 2009, 9, 320-340. [CrossRef] [PubMed]

255. Wilson, T.; Johnston, P.; Longley, D. Anti-Apoptotic Mechanisms of Drug Resistance in Cancer. Curr. Cancer Drug Targets 2009, 9 , 307-319. [CrossRef]

256. Goodwin, C.M.; Rossanese, O.W.; Olejniczak, E.T.; Fesik, S.W. Myeloid Cell Leukemia-1 Is an Important Apoptotic Survival Factor in Triple-Negative Breast Cancer. Cell Death Differ. 2015, 22, 2098-2106. [CrossRef] [PubMed]

257. Ozretic, P.; Alvir, I.; Sarcevic, B.; Vujaskovic, Z.; Rendic-Miocevic, Z.; Roguljic, A.; Beketic-Oreskovic, L. Apoptosis Regulator Bcl-2 Is an Independent Prognostic Marker for Worse Overall Survival in Triple-Negative Breast Cancer Patients. Int. J. Biol. Markers 2017, 33, 109-115. [CrossRef] 
258. Zoeller, J.J.; Vagodny, A.; Daniels, V.W.; Taneja, K.; Tan, B.Y.; DeRose, Y.S.; Fujita, M.; Welm, A.L.; Letai, A.; Leverson, J.D.; et al. Navitoclax Enhances the Effectiveness of EGFR-Targeted Antibody-Drug Conjugates in PDX Models of EGFR-Expressing Triple-Negative Breast Cancer. Breast Cancer Res. 2020, 22, 132. [CrossRef] [PubMed]

259. Hiraki, M.; Suzuki, Y.; Alam, M.; Hinohara, K.; Hasegawa, M.; Jin, C.; Kharbanda, S.; Kufe, D. MUC1-C Stabilizes MCL-1 in the Oxidative Stress Response of Triple-Negative Breast Cancer Cells to BCL-2 Inhibitors. Sci. Rep. 2016, 6, 26643. [CrossRef]

260. Cruz-Gordillo, P.; Honeywell, M.E.; Harper, N.W.; Leete, T.; Lee, M.J. ELP-Dependent Expression of MCL1 Promotes Resistance to EGFR Inhibition in Triple-Negative Breast Cancer Cells. Sci. Signal. 2020, 13, eabb9820. [CrossRef]

261. Chen, M.J.; Dixon, J.E.; Manning, G. Genomics and Evolution of Protein Phosphatases. Sci. Signal. 2017, 10, eaag1796. [CrossRef] [PubMed]

262. Sun, T.; Aceto, N.; Meerbrey, K.L.; Kessler, J.D.; Zhou, C.; Migliaccio, I.; Nguyen, D.X.; Pavlova, N.N.; Botero, M.; Huang, J.; et al. Activation of Multiple Proto-Oncogenic Tyrosine Kinases in Breast Cancer via Loss of the PTPN12 Phosphatase. Cell 2011, 144, 703-718. [CrossRef] [PubMed]

263. Ahmed, T.A.; Adamopoulos, C.; Karoulia, Z.; Wu, X.; Sachidanandam, R.; Aaronson, S.A.; Poulikakos, P.I. SHP2 Drives Adaptive Resistance to ERK Signaling Inhibition in Molecularly Defined Subsets of ERK-Dependent Tumors. Cell Rep. 2019, 26, 65-78.e5. [CrossRef]

264. Matalkah, F.; Martin, E.; Zhao, H.; Agazie, Y.M. SHP2 Acts Both Upstream and Downstream of Multiple Receptor Tyrosine Kinases to Promote Basal-like and Triple-Negative Breast Cancer. Breast Cancer Res. 2016, 18, 2. [CrossRef] [PubMed]

265. Chen, H.; Libring, S.; Ruddraraju, K.V.; Miao, J.; Solorio, L.; Zhang, Z.-Y.; Wendt, M.K. SHP2 Is a Multifunctional Therapeutic Target in Drug Resistant Metastatic Breast Cancer. Oncogene 2020, 39, 7166-7180. [CrossRef]

266. Song, Z.-D.; Wang, M.-J.; Ge, Y.; Chen, X.-P.; Xu, Z.-Y.; Sun, Y.; Xiong, X.-F. Tyrosine Phosphatase SHP2 Inhibitors in TumorTargeted Therapies. Acta Pharm. Sin. B 2020, 11, 13-29. [CrossRef]

267. Yuan, X.; Bu, H.; Zhou, J.; Yang, C.-Y.; Zhang, H. Recent Advances of SHP2 Inhibitors in Cancer Therapy: Current Development and Clinical Application. J. Med. Chem. 2020, 63, 11368-11396. [CrossRef]

268. Whitesell, L.; Lindquist, S.L. HSP90 and the Chaperoning of Cancer. Nat. Rev. Cancer 2005, 5, 761-772. [CrossRef] [PubMed]

269. Mayer, M.P.; Le Breton, L. Hsp90: Breaking the Symmetry. Mol. Cell 2015, 58, 8-20. [CrossRef] [PubMed]

270. Röhl, A.; Rohrberg, J.; Buchner, J. The Chaperone Hsp90: Changing Partners for Demanding Clients. Trends Biochem. Sci. 2013, 38, 253-262. [CrossRef] [PubMed]

271. Sankhala, K.K.; Mita, M.M.; Mita, A.C.; Takimoto, C.H. Heat Shock Proteins: A Potential Anticancer Target. Curr. Drug Targets 2011, 12, 2001-2008. [CrossRef] [PubMed]

272. Kim, T.-S.; Jang, C.-Y.; Kim, H.D.; Lee, J.Y.; Ahn, B.-Y.; Kim, J. Interaction of Hsp90 with Ribosomal Proteins Protects from Ubiquitination and Proteasome-Dependent Degradation. Mol. Biol. Cell 2006, 17, 824-833. [CrossRef] [PubMed]

273. Mumin, N.H.; Drobnitzky, N.; Patel, A.; Lourenco, L.M.; Cahill, F.F.; Jiang, Y.; Kong, A.; Ryan, A.J. Overcoming Acquired Resistance to HSP90 Inhibition by Targeting JAK-STAT Signalling in Triple-Negative Breast Cancer. BMC Cancer 2019, 19, 102. [CrossRef] [PubMed]

274. Wang, Z.; Zhang, X.; Shen, P.; Loggie, B.W.; Chang, Y.; Deuel, T.F. Identification, Cloning, and Expression of Human Estrogen Receptor-A36, a Novel Variant of Human Estrogen Receptor-A66. Biochem. Biophys Res. Commun 2005, 336, 1023-1027. [CrossRef] [PubMed]

275. Thiebaut, C.; Konan, H.-P.; Guerquin, M.-J.; Chesnel, A.; Livera, G.; Romancer, M.L.; Dumond, H. The Role of ER $\alpha 36$ in Development and Tumor Malignancy. Int. J. Mol. Sci. 2020, 21, 4116. [CrossRef] [PubMed]

276. Mahboobifard, F.; Dargahi, L.; Jorjani, M.; Tehrani, F.R.; Pourgholami, M.H. The Role of ER $\alpha 36$ in Cell Type-Specific Functions of Estrogen and Cancer Development. Pharm. Res. 2021, 163, 105307. [CrossRef] [PubMed]

277. Wang, Z.; Zhang, X.; Shen, P.; Loggie, B.W.; Chang, Y.; Deuel, T.F. A Variant of Estrogen Receptor- $\alpha$, HER-A36: Transduction of Estrogen- and Antiestrogen-Dependent Membrane-Initiated Mitogenic Signaling. Proc. Natl Acad. Sci. USA 2006, 103, 9063-9068. [CrossRef]

278. Zhang, X.T.; Kang, L.G.; Ding, L.; Vranic, S.; Gatalica, Z.; Wang, Z.-Y. A Positive Feedback Loop of ER-A36/EGFR Promotes Malignant Growth of ER-Negative Breast Cancer Cells. Oncogene 2011, 30, 770-780. [CrossRef]

279. Jordan, V.C. Tamoxifen (ICI46,474) as a Targeted Therapy to Treat and Prevent Breast Cancer. Br. J. Pharm. 2006, 147, S269-S276. [CrossRef]

280. Wang, Q.; Jiang, J.; Ying, G.; Xie, X.-Q.; Zhang, X.; Xu, W.; Zhang, X.; Song, E.; Bu, H.; Ping, Y.-F.; et al. Tamoxifen Enhances Stemness and Promotes Metastasis of ER $\alpha 36+$ Breast Cancer by Upregulating ALDH1A1 in Cancer Cells. Cell Res. 2018, 28, 336-358. [CrossRef]

281. Xu, X.; Wang, Q.; He, Y.; Ding, L.; Zhong, F.; Ou, Y.; Shen, Y.; Liu, H.; He, S. ADP-Ribosylation Factor 1 (ARF1) Takes Part in Cell Proliferation and Cell Adhesion-Mediated Drug Resistance (CAM-DR). Ann. Hematol. 2017, 96, 847-858. [CrossRef]

282. Lang, L.; Shay, C.; Zhao, X.; Teng, Y. Combined Targeting of Arf1 and Ras Potentiates Anticancer Activity for Prostate Cancer Therapeutics. J. Exp. Clin. Cancer Res. 2017, 36, 112. [CrossRef]

283. Gu, G.; Chen, Y.; Duan, C.; Zhou, L.; Chen, C.; Chen, J.; Cheng, J.; Shi, N.; Jin, Y.; Xi, Q.; et al. Overexpression of ARF1 Is Associated with Cell Proliferation and Migration through PI3K Signal Pathway in Ovarian Cancer. Oncol. Rep. 2017, 37, 1511-1520. [CrossRef] 
284. Luchsinger, C.; Aguilar, M.; Burgos, P.V.; Ehrenfeld, P.; Mardones, G.A. Functional Disruption of the Golgi Apparatus Protein ARF1 Sensitizes MDA-MB-231 Breast Cancer Cells to the Antitumor Drugs Actinomycin D and Vinblastine through ERK and AKT Signaling. PLoS ONE 2018, 13, e0195401. [CrossRef]

285. Vo-Hoang, Y.; Paiva, S.; He, L.; Estaran, S.; Teng, Y. Design and Synthesis of Arf1-Targeting $\gamma$-Dipeptides as Potential Agents against Head and Neck Squamous Cell Carcinoma. Cells 2020, 9, 286. [CrossRef]

286. Gillingham, A.K.; Munro, S. The Small G Proteins of the Arf Family and Their Regulators. Annu. Rev. Cell Dev. Biol. 2007, 23, 579-611. [CrossRef]

287. Casalou, C.; Ferreira, A.; Barral, D.C. The Role of ARF Family Proteins and Their Regulators and Effectors in Cancer Progression: A Therapeutic Perspective. Front. Cell Dev. Biol. 2020, 8, 217. [CrossRef]

288. Haines, E.; Schlienger, S.; Claing, A. The Small GTPase ADP-Ribosylation Factor 1 Mediates the Sensitivity of Triple Negative Breast Cancer Cells to EGFR Tyrosine Kinase Inhibitors. Cancer Biol. 2015, 16, 1535-1547. [CrossRef] [PubMed]

289. Sauer, H.; Wartenberg, M.; Hescheler, J. Reactive Oxygen Species as Intracellular Messengers During Cell Growth and Differentiation. Cell Physiol. Biochem. 2001, 11, 173-186. [CrossRef]

290. Cui, Q.; Wang, J.-Q.; Assaraf, Y.G.; Ren, L.; Gupta, P.; Wei, L.; Ashby, C.R.; Yang, D.-H.; Chen, Z.-S. Modulating ROS to Overcome Multidrug Resistance in Cancer. Drug Resist. Updates 2018, 41, 1-25. [CrossRef] [PubMed]

291. Weng, M.-S.; Chang, J.-H.; Hung, W.-Y.; Yang, Y.-C.; Chien, M.-H. The Interplay of Reactive Oxygen Species and the Epidermal Growth Factor Receptor in Tumor Progression and Drug Resistance. J. Exp. Clin. Cancer Res. 2018, 37, 61. [CrossRef] [PubMed]

292. Bhardwaj, V.; He, J. Reactive Oxygen Species, Metabolic Plasticity, and Drug Resistance in Cancer. Int. J. Mol. Sci. 2020, $21,3412$. [CrossRef]

293. Chen, C.-H.; Cheng, T.-H.; Lin, H.; Shih, N.-L.; Chen, Y.-L.; Chen, Y.-S.; Cheng, C.-F.; Lian, W.-S.; Meng, T.-C.; Chiu, W.-T.; et al. Reactive Oxygen Species Generation Is Involved in Epidermal Growth Factor Receptor Transactivation through the Transient Oxidization of Src Homology 2-Containing Tyrosine Phosphatase in Endothelin-1 Signaling Pathway in Rat Cardiac Fibroblasts. Mol. Pharm. 2006, 69, 1347-1355. [CrossRef]

294. Gallatin, W.M.; Weissman, I.L.; Butcher, E.C. A Cell-Surface Molecule Involved in Organ-Specific Homing of Lymphocytes. Nature 1983, 304, 30-34. [CrossRef]

295. Lesley, J.; Hyman, R.; Kincade, P.W. CD44 and Its Interaction with Extracellular Matrix. Adv. Immunol. 1993, 54, 271-335. [CrossRef]

296. Peach, R.; Hollenbaugh, D.; Stamenkovic, I.; Aruffo, A. Identification of Hyaluronic Acid Binding Sites in the Extracellular Domain of CD44. J. Cell Biol. 1993, 122, 257-264. [CrossRef]

297. Faassen, A.E.; Schrager, J.A.; Klein, D.J.; Oegema, T.R.; Couchman, J.R.; McCarthy, J.B. A Cell Surface Chondroitin Sulfate Proteoglycan, Immunologically Related to CD44, Is Involved in Type I Collagen-Mediated Melanoma Cell Motility and Invasion. J. Cell Biol. 1992, 116, 521-531. [CrossRef]

298. Knutson, J.R.; Iida, J.; Fields, G.B.; McCarthy, J.B. CD44/Chondroitin Sulfate Proteoglycan and Alpha 2 Beta 1 Integrin Mediate Human Melanoma Cell Migration on Type IV Collagen and Invasion of Basement Membranes. Mol. Biol. Cell 1996, 7, 383-396. [CrossRef] [PubMed]

299. Dimitroff, C.J.; Lee, J.Y.; Rafii, S.; Fuhlbrigge, R.C.; Sackstein, R. CD44 Is a Major E-Selectin Ligand on Human Hematopoietic Progenitor Cells. J. Cell Biol. 2001, 153, 1277-1286. [CrossRef] [PubMed]

300. Katayama, Y.; Hidalgo, A.; Chang, J.; Peired, A.; Frenette, P.S. CD44 Is a Physiological E-Selectin Ligand on Neutrophils. J. Exp. Med. 2005, 201, 1183-1189. [CrossRef] [PubMed]

301. Jalkanen, S.; Jalkanen, M. Lymphocyte CD44 Binds the COOH-Terminal Heparin-Binding Domain of Fibronectin. J. Cell Biol. 1992, 116, 817-825. [CrossRef] [PubMed]

302. Bourguignon, L.Y.W.; Gunja-Smith, Z.; Iida, N.; Zhu, H.B.; Young, L.J.T.; Muller, W.J.; Cardiff, R.D. CD44v3,8-10 Is Involved in Cytoskeleton-mediated Tumor Cell Migration and Matrix Metalloproteinase (MMP-9) Association in Metastatic Breast Cancer Cells. J. Cell Physiol. 1998, 176, 206-215. [CrossRef]

303. Weber, G.F.; Ashkar, S.; Glimcher, M.J.; Cantor, H. Receptor-Ligand Interaction Between CD44 and Osteopontin (Eta-1). Science 1996, 271, 509-512. [CrossRef]

304. Haynes, B.F.; Telen, M.J.; Hale, L.P.; Denning, S.M. CD44-A Molecule Involved in Leukocyte Adherence and T-Cell Activation. Immunol. Today 1989, 10, 423-428. [CrossRef]

305. Fukazawa, H.; Yoshida, K.; Ichinohasama, R.; Sawai, T.; Hiromatsu, Y.; Mori, K.; Kikuchi, K.; Aizawa, Y.; Abe, K.; Wall, J.R. Expression of the Hermes-1 (CD44) and ICAM-1 (CD54) Molecule on the Surface of Thyroid Cells from Patients with Graves' Disease. Thyroid 1993, 3, 285-289. [CrossRef] [PubMed]

306. Deguchi, T.; Komada, Y. Homing-Associated Cell Adhesion Molecule (H-CAM/CD44) on Human CD34+ Hematopoietic Progenitor Cells. Leuk. Lymphoma 2000, 40, 25-37. [CrossRef]

307. Joensuu, H.; Ristamäki, R.; Klemi, P.; Jalkanen, S. Lymphocyte Homing Receptor (CD44) Expression Is Associated with Poor Prognosis in Gastrointestinal Lymphoma. Br. J. Cancer 1993, 68, 428-432. [CrossRef] [PubMed]

308. Raso-Barnett, L.; Banky, B.; Barbai, T.; Becsagh, P.; Timar, J.; Raso, E. Demonstration of a Melanoma-Specific CD44 Alternative Splicing Pattern That Remains Qualitatively Stable, but Shows Quantitative Changes during Tumour Progression. PLoS ONE 2013, 8, e53883. [CrossRef] 
309. Chen, C.; Zhao, S.; Karnad, A.; Freeman, J.W. The Biology and Role of CD44 in Cancer Progression: Therapeutic Implications. J. Hematol. Oncol. 2018, 11, 64. [CrossRef]

310. Lee, J.H.; Ha, D.H.; Go, H.; Youn, J.; Kim, H.; Jin, R.C.; Miller, R.B.; Kim, D.; Cho, B.S.; Yi, Y.W. Reproducible Large-Scale Isolation of Exosomes from Adipose Tissue-Derived Mesenchymal Stem/Stromal Cells and Their Application in Acute Kidney Injury. Int. J. Mol. Sci. 2020, 21, 4774. [CrossRef] [PubMed]

311. Shin, K.-O.; Ha, D.H.; Kim, J.O.; Crumrine, D.A.; Meyer, J.M.; Wakefield, J.S.; Lee, Y.; Kim, B.; Kim, S.; Kim, H.; et al. Exosomes from Human Adipose Tissue-Derived Mesenchymal Stem Cells Promote Epidermal Barrier Repair by Inducing de Novo Synthesis of Ceramides in Atopic Dermatitis. Cells 2020, 9, 680. [CrossRef] [PubMed]

312. Cho, B.S.; Lee, J.; Won, Y.; Duncan, D.I.; Jin, R.C.; Lee, J.; Kwon, H.H.; Park, G.-H.; Yang, S.H.; Park, B.C.; et al. Skin Brightening Efficacy of Exosomes Derived from Human Adipose Tissue-Derived Stem/Stromal Cells: A Prospective, Split-Face, Randomized Placebo-Controlled Study. Cosmetics 2020, 7, 90. [CrossRef]

313. Xu, H.; Niu, M.; Yuan, X.; Wu, K.; Liu, A. CD44 as a Tumor Biomarker and Therapeutic Target. Exp. Hematol. Oncol. 2020, 9 , 36. [CrossRef]

314. Jaggupilli, A.; Elkord, E. Significance of CD44 and CD24 as Cancer Stem Cell Markers: An Enduring Ambiguity. Clin. Dev. Immunol. 2012, 2012, 708036. [CrossRef]

315. Yin, J.; Zhang, H.; Wu, X.; Zhang, Y.; Li, J.; Shen, J.; Zhao, Y.; Xiao, Z.; Lu, L.; Huang, C.; et al. CD44 Inhibition Attenuates EGFR Signaling and Enhances Cisplatin Sensitivity in Human EGFR Wild-Type Non-Small-Cell Lung Cancer Cells. Int. J. Mol. Med. 2020, 45, 1783-1792. [CrossRef]

316. Fu, W.; Sun, H.; Zhao, Y.; Chen, M.; Yang, L.; Yang, X.; Jin, W. Targeted Delivery of CD44s-SiRNA by ScFv Overcomes de Novo Resistance to Cetuximab in Triple Negative Breast Cancer. Mol. Immunol. 2018, 99, 124-133. [CrossRef]

317. Anborgh, P.H.; Lee, D.J.; Stam, P.F.; Tuck, A.B.; Chambers, A.F. Role of Osteopontin as a Predictive Biomarker for Anti-EGFR Therapy in Triple-Negative Breast Cancer. Expert Opin. Targets 2018, 22, 727-734. [CrossRef] [PubMed]

318. Tuck, A.B.; Hota, C.; Wilson, S.M.; Chambers, A.F. Osteopontin-Induced Migration of Human Mammary Epithelial Cells Involves Activation of EGF Receptor and Multiple Signal Transduction Pathways. Oncogene 2003, 22, 1198-1205. [CrossRef] [PubMed]

319. Singhal, H.; Bautista, D.S.; Tonkin, K.S.; O'Malley, F.P.; Tuck, A.B.; Chambers, A.F.; Harris, J.F. Elevated Plasma Osteopontin in Metastatic Breast Cancer Associated with Increased Tumor Burden and Decreased Survival. Clin. Cancer Res. 1997, 3, 605-611. [PubMed]

320. Tuck, A.B.; O’Malley, F.P.; Singhal, H.; Harris, J.F.; Tonkin, K.S.; Kerkvliet, N.; Saad, Z.; Doig, G.S.; Chambers, A.F. Osteopontin Expression in a Group of Lymph Node Negative Breast Cancer Patients. Int. J. Cancer 1998, 79, 502-508. [CrossRef]

321. Bramwell, V.H.C.; Doig, G.S.; Tuck, A.B.; Wilson, S.M.; Tonkin, K.S.; Tomiak, A.; Perera, F.; Vandenberg, T.A.; Chambers, A.F. Serial Plasma Osteopontin Levels Have Prognostic Value in Metastatic Breast Cancer. Clin. Cancer Res. 2006, 12, $3337-3343$. [CrossRef] [PubMed]

322. Anborgh, P.H.; Caria, L.B.; Chambers, A.F.; Tuck, A.B.; Stitt, L.W.; Brackstone, M. Role of Plasma Osteopontin as a Biomarker in Locally Advanced Breast Cancer. Am. J. Transl. Res. 2015, 7, 723-732. [PubMed]

323. Xu, Y.-Y.; Zhang, Y.-Y.; Lu, W.-F.; Mi, Y.-J.; Chen, Y.-Q. Prognostic Value of Osteopontin Expression in Breast Cancer: A MetaAnalysis. Mol. Clin. Oncol. 2015, 3, 357-362. [CrossRef] [PubMed]

324. Zhang, Y.; Bi, J.; Zhu, H.; Shi, M.; Zeng, X. ANXA2 Could Act as a Moderator of EGFR-Directed Therapy Resistance in Triple Negative Breast Cancer. Biosci. Biotechnol. Biochem. 2018, 82, 1-9. [CrossRef]

325. Christensen, M.V.; Høgdall, C.K.; Jochumsen, K.M.; Høgdall, E.V.S. Annexin A2 and Cancer: A Systematic Review. Int. J. Oncol. 2018, 52, 5-18. [CrossRef] [PubMed]

326. Wang, T.; Wang, Z.; Niu, R.; Wang, L. Crucial Role of Anxa2 in Cancer Progression: Highlights on Its Novel Regulatory Mechanism. Cancer Biol. Med. 2019, 16, 671-687. [CrossRef] [PubMed]

327. Shetty, P.K.; Thamake, S.I.; Biswas, S.; Johansson, S.L.; Vishwanatha, J.K. Reciprocal Regulation of Annexin A2 and EGFR with Her-2 in Her-2 Negative and Herceptin-Resistant Breast Cancer. PLoS ONE 2012, 7, e44299. [CrossRef]

328. De Graauw, M.; Cao, L.; Winkel, L.; van Miltenburg, M.H.A.M.; le Dévédec, S.E.; Klop, M.; Yan, K.; Pont, C.; Rogkoti, V.-M.; Tijsma, A.; et al. Annexin A2 Depletion Delays EGFR Endocytic Trafficking via Cofilin Activation and Enhances EGFR Signaling and Metastasis Formation. Oncogene 2014, 33, 2610-2619. [CrossRef]

329. Klionsky, D.J. Autophagy Revisited: A Conversation with Christian de Duve. Autophagy 2008, 4, 740-743. [CrossRef] [PubMed]

330. Mizushima, N.; Komatsu, M. Autophagy: Renovation of Cells and Tissues. Cell 2011, 147, 728-741. [CrossRef] [PubMed]

331. Li, X.; Zhou, Y.; Li, Y.; Yang, L.; Ma, Y.; Peng, X.; Yang, S.; Liu, J.; Li, H. Autophagy: A Novel Mechanism of Chemoresistance in Cancers. Biomed. Pharm. 2019, 119, 109415. [CrossRef]

332. Chang, H.; Zou, Z. Targeting Autophagy to Overcome Drug Resistance: Further Developments. J. Hematol. Oncol. 2020, 13, 159. [CrossRef] [PubMed]

333. Dragowska, W.H.; Weppler, S.A.; Wang, J.C.; Wong, L.Y.; Kapanen, A.I.; Rawji, J.S.; Warburton, C.; Qadir, M.A.; Donohue, E.; Roberge, M.; et al. Induction of Autophagy Is an Early Response to Gefitinib and a Potential Therapeutic Target in Breast Cancer. PLoS ONE 2013, 8, e76503. [CrossRef]

334. Li, X.; Fan, Z. The Epidermal Growth Factor Receptor Antibody Cetuximab Induces Autophagy in Cancer Cells by Downregulating HIF-1 $\alpha$ and Bcl-2 and Activating the Beclin 1/HVps34 Complex. Cancer Res. 2010, 70, 5942-5952. [CrossRef] [PubMed] 
335. Wei, Y.; Zou, Z.; Becker, N.; Anderson, M.; Sumpter, R.; Xiao, G.; Kinch, L.; Koduru, P.; Christudass, C.S.; Veltri, R.W.; et al. EGFR-Mediated Beclin 1 Phosphorylation in Autophagy Suppression, Tumor Progression, and Tumor Chemoresistance. Cell 2013, 154, 1269-1284. [CrossRef] [PubMed]

336. Jutten, B.; Rouschop, K. EGFR Signaling and Autophagy Dependence for Growth, Survival, and Therapy Resistance. Cell Cycle 2014, 13, 42-51. [CrossRef]

337. Fung, C.; Chen, X.; Grandis, J.R.; Duvvuri, U. EGFR Tyrosine Kinase Inhibition Induces Autophagy in Cancer Cells. Cancer Biol. 2012, 13, 1417-1424. [CrossRef] [PubMed]

338. Tan, X.; Thapa, N.; Sun, Y.; Anderson, R.A. A Kinase-Independent Role for EGF Receptor in Autophagy Initiation. Cell 2015, 160, 145-160. [CrossRef]

339. Rontogianni, S.; Iskit, S.; van Doorn, S.; Peeper, D.S.; Altelaar, M. Combined EGFR and ROCK Inhibition in Triple-Negative Breast Cancer Leads to Cell Death Via Impaired Autophagic Flux. Mol. Cell Proteom. 2020, 19, 261-277. [CrossRef]

340. Jones, V.S.; Huang, R.-Y.; Chen, L.-P.; Chen, Z.-S.; Fu, L.; Huang, R.-P. Cytokines in Cancer Drug Resistance: Cues to New Therapeutic Strategies. Biochim. Biophys. Acta 2016, 1865, 255-265. [CrossRef]

341. Chen, W.; Qin, Y.; Liu, S. Cytokines, Breast Cancer Stem Cells (BCSCs) and Chemoresistance. Clin. Transl. Med. 2018, 7, 27. [CrossRef]

342. Jena, B.C.; Mandal, M. The Emerging Roles of Exosomes in Anti-Cancer Drug Resistance and Tumor Progression: An Insight towards Tumor-Microenvironment Interaction. Biochim. Biophys. Acta 2021, 1875, 188488. [CrossRef]

343. Namee, N.M.; O’Driscoll, L. Extracellular Vesicles and Anti-Cancer Drug Resistance. Biochim. Biophys. Acta 2018, 1870, 123-136. [CrossRef]

344. Straussman, R.; Morikawa, T.; Shee, K.; Barzily-Rokni, M.; Qian, Z.R.; Du, J.; Davis, A.; Mongare, M.M.; Gould, J.; Frederick, D.T.; et al. Tumour Micro-Environment Elicits Innate Resistance to RAF Inhibitors through HGF Secretion. Nature 2012, 487, 500-504. [CrossRef] [PubMed]

345. Rickel, E.A.; Siegel, L.A.; Yoon, B.-R.P.; Rottman, J.B.; Kugler, D.G.; Swart, D.A.; Anders, P.M.; Tocker, J.E.; Comeau, M.R.; Budelsky, A.L. Identification of Functional Roles for Both IL-17RB and IL-17RA in Mediating IL-25-Induced Activities. J. Immunol. 2008, 181, 4299-4310. [CrossRef] [PubMed]

346. Reynolds, J.M.; Lee, Y.-H.; Shi, Y.; Wang, X.; Angkasekwinai, P.; Nallaparaju, K.C.; Flaherty, S.; Chang, S.H.; Watarai, H.; Dong, C. Interleukin-17B Antagonizes Interleukin-25-Mediated Mucosal Inflammation. Immunity 2015, 42, 692-703. [CrossRef]

347. Merrouche, Y.; Fabre, J.; Cure, H.; Garbar, C.; Fuselier, C.; Bastid, J.; Antonicelli, F.; Al-Daccak, R.; Bensussan, A.; Giustiniani, J. IL-17E Synergizes with EGF and Confers in Vitro Resistance to EGFR-Targeted Therapies in TNBC Cells. Oncotarget 2016, 7, 53350-53361. [CrossRef] [PubMed]

348. Kalluri, R.; LeBleu, V.S. The Biology, Function, and Biomedical Applications of Exosomes. Science 2020, 367, eaau6977. [CrossRef] [PubMed]

349. Dong, X.; Bai, X.; Ni, J.; Zhang, H.; Duan, W.; Graham, P.; Li, Y. Exosomes and Breast Cancer Drug Resistance. Cell Death Dis. 2020, 11, 987. [CrossRef] [PubMed]

350. Goh, C.Y.; Wyse, C.; Ho, M.; O’Beirne, E.; Howard, J.; Lindsay, S.; Kelly, P.; Higgins, M.; McCann, A. Exosomes in Triple Negative Breast Cancer: Garbage Disposals or Trojan Horses? Cancer Lett. 2020, 473, 90-97. [CrossRef] [PubMed]

351. Scheffler, M.; Merkelbach-Bruse, S.; Bos, M.; Fassunke, J.; Gardizi, M.; Michels, S.; Groneck, L.; Schultheis, A.M.; Malchers, F.; Leenders, F; et al. Spatial Tumor Heterogeneity in Lung Cancer with Acquired Epidermal Growth Factor Receptor-Tyrosine Kinase Inhibitor Resistance: Targeting High-Level MET-Amplification and EGFR T790M Mutation Occurring at Different Sites in the Same Patient. J. Thorac. Oncol. 2015, 10, e40-e43. [CrossRef] [PubMed]

352. Nishio, M.; Horiike, A.; Murakami, H.; Yamamoto, N.; Kaneda, H.; Nakagawa, K.; Horinouchi, H.; Nagashima, M.; Sekiguchi, M.; Tamura, T. Phase I Study of the HER3-Targeted Antibody Patritumab (U3-1287) Combined with Erlotinib in Japanese Patients with Non-Small Cell Lung Cancer. Lung Cancer 2015, 88, 275-281. [CrossRef]

353. Reckamp, K.L.; Koczywas, M.; Cristea, M.C.; Dowell, J.E.; Wang, H.; Gardner, B.K.; Milne, G.L.; Figlin, R.A.; Fishbein, M.C.; Elashoff, R.M.; et al. Randomized Phase 2 Trial of Erlotinib in Combination with High-dose Celecoxib or Placebo in Patients with Advanced Non-small Cell Lung Cancer. Cancer 2015, 121, 3298-3306. [CrossRef]

354. Lee, J.Y.; Sun, J.-M.; Lim, S.H.; Kim, H.S.; Yoo, K.H.; Jung, K.S.; Song, H.-N.; Ku, B.M.; Koh, J.; Bae, Y.-H.; et al. A Phase Ib/II Study of Afatinib in Combination with Nimotuzumab in Non-Small Cell Lung Cancer Patients with Acquired Resistance to Gefitinib or Erlotinib. Clin. Cancer Res. 2016, 22, 2139-2145. [CrossRef] [PubMed]

355. Zwitter, M.; Rajer, M.; Stanic, K.; Vrankar, M.; Doma, A.; Cuderman, A.; Grmek, M.; Kern, I.; Kovac, V. Intercalated Chemotherapy and Erlotinib for Non-Small Cell Lung Cancer (NSCLC) with Activating Epidermal Growth Factor Receptor (EGFR) Mutations. Cancer Biol. 2016, 17, 1-7. [CrossRef] [PubMed]

356. Rosell, R.; Dafni, U.; Felip, E.; Curioni-Fontecedro, A.; Gautschi, O.; Peters, S.; Massutí, B.; Palmero, R.; Aix, S.P.; Carcereny, E.; et al. Erlotinib and Bevacizumab in Patients with Advanced Non-Small-Cell Lung Cancer and Activating EGFR Mutations (BELIEF): An International, Multicentre, Single-Arm, Phase 2 Trial. Lancet Respir. Med. 2017, 5, 435-444. [CrossRef]

357. Wu, Y.-L.; Zhang, L.; Kim, D.-W.; Liu, X.; Lee, D.H.; Yang, J.C.-H.; Ahn, M.-J.; Vansteenkiste, J.F.; Su, W.-C.; Felip, E.; et al. Phase $\mathrm{Ib} / \mathrm{II}$ Study of Capmatinib (INC280) Plus Gefitinib After Failure of Epidermal Growth Factor Receptor (EGFR) Inhibitor Therapy in Patients with EGFR-Mutated, MET Factor-Dysregulated Non-Small-Cell Lung Cancer. J. Clin. Oncol. 2018, 36, 3101-3109. [CrossRef] [PubMed] 
358. Hata, A.; Katakami, N.; Kaji, R.; Yokoyama, T.; Kaneda, T.; Tamiya, M.; Inoue, T.; Kimura, H.; Yano, Y.; Tamura, D.; et al. Afatinib plus Bevacizumab Combination after Acquired Resistance to EGFR Tyrosine Kinase Inhibitors in EGFR-mutant Non-Small Cell Lung Cancer: Multicenter, Single-arm, Phase 2 Trial (ABC Study). Cancer 2018, 124, 3830-3838. [CrossRef] [PubMed]

359. Uchibori, K.; Satouchi, M.; Sueoka-Aragane, N.; Urata, Y.; Sato, A.; Imamura, F.; Inoue, T.; Tachihara, M.; Kobayashi, K.; Katakami, N.; et al. Phase II Trial of Gefitinib plus Pemetrexed after Relapse Using First-Line Gefitinib in Patients with Non-Small Cell Lung Cancer Harboring EGFR Gene Mutations. Lung Cancer 2018, 124, 65-70. [CrossRef]

360. Park, J.S.; Hong, M.H.; Chun, Y.J.; Kim, H.R.; Cho, B.C. A Phase Ib Study of the Combination of Afatinib and Ruxolitinib in EGFR Mutant NSCLC with Progression on EGFR-TKIs. Lung Cancer 2019, 134, 46-51. [CrossRef]

361. Yang, J.C.-H.; Cheng, Y.; Murakami, H.; Yang, P.-C.; He, J.; Nakagawa, K.; Kang, J.H.; Kim, J.-H.; Hozak, R.R.; Nguyen, T.S.; et al A Randomized Phase 2 Study of Gefitinib with or without Pemetrexed as First-Line Treatment in Nonsquamous NSCLC with EGFR Mutation: Final Overall Survival and Biomarker Analysis. J. Thorac. Oncol. 2020, 15, 91-100. [CrossRef]

362. Wu, Y.-L.; Cheng, Y.; Zhou, J.; Lu, S.; Zhang, Y.; Zhao, J.; Kim, D.-W.; Soo, R.A.; Kim, S.-W.; Pan, H.; et al. Tepotinib plus Gefitinib in Patients with EGFR-Mutant Non-Small-Cell Lung Cancer with MET Overexpression or MET Amplification and Acquired Resistance to Previous EGFR Inhibitor (INSIGHT Study): An Open-Label, Phase 1b/2, Multicentre, Randomised Trial. Lancet Respir. Med. 2020, 8, 1132-1143. [CrossRef]

363. Sequist, L.V.; Han, J.-Y.; Ahn, M.-J.; Cho, B.C.; Yu, H.; Kim, S.-W.; Yang, J.C.-H.; Lee, J.S.; Su, W.-C.; Kowalski, D.; et al. Osimertinib plus Savolitinib in Patients with EGFR Mutation-Positive, MET-Amplified, Non-Small-Cell Lung Cancer after Progression on EGFR Tyrosine Kinase Inhibitors: Interim Results from a Multicentre, Open-Label, Phase 1b Study. Lancet Oncol. 2020, 21, 373-386. [CrossRef]

364. Matar, P.; Rojo, F.; Cassia, R.; Moreno-Bueno, G.; Cosimo, S.D.; Tabernero, J.; Guzmán, M.; Rodriguez, S.; Arribas, J.; Palacios, J.; et al. Combined Epidermal Growth Factor Receptor Targeting with the Tyrosine Kinase Inhibitor Gefitinib (ZD1839) and the Monoclonal Antibody Cetuximab (IMC-C225) Superiority Over Single-Agent Receptor Targeting. Clin. Cancer Res. 2004, 10, 6487-6501. [CrossRef]

365. Oliveras-Ferraros, C.; Vazquez-Martin, A.; López-Bonet, E.; Martín-Castillo, B.; Barco, S.D.; Brunet, J.; Menendez, J.A. Growth and Molecular Interactions of the Anti-EGFR Antibody Cetuximab and the DNA Cross-Linking Agent Cisplatin in Gefitinib-Resistant MDA-MB-468 Cells: New Prospects in the Treatment of Triple-Negative/Basal-like Breast Cancer. Int. J. Oncol. 2008, 33, 1165-1176. [CrossRef]

366. Corkery, B.; Crown, J.; Clynes, M.; O’Donovan, N. Epidermal Growth Factor Receptor as a Potential Therapeutic Target in Triple-Negative Breast Cancer. Ann. Oncol. 2009, 20, 862-867. [CrossRef]

367. Edwards, S.R.; Wandless, T.J. The Rapamycin-Binding Domain of the Protein Kinase Mammalian Target of Rapamycin Is a Destabilizing Domain. J. Biol. Chem. 2007, 282, 13395-13401. [CrossRef]

368. Liu, T.; Yacoub, R.; Taliaferro-Smith, L.D.; Sun, S.-Y.; Graham, T.R.; Dolan, R.; Lobo, C.; Tighiouart, M.; Yang, L.; Adams, A.; et al. Combinatorial Effects of Lapatinib and Rapamycin in Triple-Negative Breast Cancer Cells. Mol. Cancer 2011, 10, 1460-1469. [CrossRef] [PubMed]

369. Roberti, M.P.; Barrio, M.M.; Bravo, A.I.; Rocca, Y.S.; Arriaga, J.M.; Bianchini, M.; Mordoh, J.; Levy, E.M. IL-15 and IL-2 Increase Cetuximab-Mediated Cellular Cytotoxicity against Triple Negative Breast Cancer Cell Lines Expressing EGFR. Breast Cancer Res. Treat. 2011, 130, 465-475. [CrossRef] [PubMed]

370. Roberti, M.P.; Rocca, Y.S.; Amat, M.; Pampena, M.B.; Loza, J.; Coló, F.; Fabiano, V.; Loza, C.M.; Arriaga, J.M.; Bianchini, M.; et al. IL-2- or IL-15-Activated NK Cells Enhance Cetuximab-Mediated Activity against Triple-Negative Breast Cancer in Xenografts and in Breast Cancer Patients. Breast Cancer Res. Treat. 2012, 136, 659-671. [CrossRef]

371. Donawho, C.K.; Luo, Y.; Luo, Y.; Penning, T.D.; Bauch, J.L.; Bouska, J.J.; Bontcheva-Diaz, V.D.; Cox, B.F.; DeWeese, T.L.; Dillehay, L.E.; et al. ABT-888, an Orally Active Poly(ADP-Ribose) Polymerase Inhibitor That Potentiates DNA-Damaging Agents in Preclinical Tumor Models. Clin. Cancer Res. 2007, 13, 2728-2737. [CrossRef] [PubMed]

372. Nowsheen, S.; Cooper, T.; Stanley, J.A.; Yang, E.S. Synthetic Lethal Interactions between EGFR and PARP Inhibition in Human Triple Negative Breast Cancer Cells. PLoS ONE 2012, 7, e46614. [CrossRef] [PubMed]

373. Giordano, C.R.; Mueller, K.L.; Terlecky, L.J.; Krentz, K.A.; Bollig-Fischer, A.; Terlecky, S.R.; Boerner, J.L. A Targeted Enzyme Approach to Sensitization of Tyrosine Kinase Inhibitor-Resistant Breast Cancer Cells. Exp. Cell Res. 2012, 318, $2014-2021$. [CrossRef]

374. O'Hare, T.; Walters, D.K.; Stoffregen, E.P.; Jia, T.; Manley, P.W.; Mestan, J.; Cowan-Jacob, S.W.; Lee, F.Y.; Heinrich, M.C.; Deininger, M.W.N.; et al. In Vitro Activity of Bcr-Abl Inhibitors AMN107 and BMS-354825 against Clinically Relevant Imatinib-Resistant Abl Kinase Domain Mutants. Cancer Res. 2005, 65, 4500-4505. [CrossRef] [PubMed]

375. Shah, N.P.; Lee, F.Y.; Luo, R.; Jiang, Y.; Donker, M.; Akin, C. Dasatinib (BMS-354825) Inhibits KITD816V, an Imatinib-Resistant Activating Mutation That Triggers Neoplastic Growth in Most Patients with Systemic Mastocytosis. Blood 2006, 108, $286-291$. [CrossRef] [PubMed]

376. Kim, E.M.H.; Mueller, K.; Gartner, E.; Boerner, J. Dasatinib Is Synergistic with Cetuximab and Cisplatin in Triple-Negative Breast Cancer Cells. J. Surg Res. 2013, 185, 231-239. [CrossRef] [PubMed]

377. Knight, Z.A.; Gonzalez, B.; Feldman, M.E.; Zunder, E.R.; Goldenberg, D.D.; Williams, O.; Loewith, R.; Stokoe, D.; Balla, A.; Toth, B.; et al. A Pharmacological Map of the PI3-K Family Defines a Role for P110 $\alpha$ in Insulin Signaling. Cell 2006, 125, 733-747. [CrossRef] 
378. Yi, Y.W.; Hong, W.; Kang, H.J.; Kim, H.J.; Zhao, W.; Wang, A.; Seong, Y.; Bae, I. Inhibition of the PI3K/AKT Pathway Potentiates Cytotoxicity of EGFR Kinase Inhibitors in Triple-negative Breast Cancer Cells. J. Cell Mol. Med. 2013, 17, 648-656. [CrossRef]

379. Adams, J.; Palombella, V.J.; Sausville, E.A.; Johnson, J.; Destree, A.; Lazarus, D.D.; Maas, J.; Pien, C.S.; Prakash, S.; Elliott, P.J. Proteasome Inhibitors: A Novel Class of Potent and Effective Antitumor Agents. Cancer Res. 1999, 59, 2615-2622. [PubMed]

380. Chen, Y.-J.; Yeh, M.-H.; Yu, M.-C.; Wei, Y.-L.; Chen, W.-S.; Chen, J.-Y.; Shih, C.-Y.; Tu, C.-Y.; Chen, C.-H.; Hsia, T.-C.; et al. Lapatinib-Induced NF-KappaB Activation Sensitizes Triple-Negative Breast Cancer Cells to Proteasome Inhibitors. Breast Cancer Res. 2013, 15, R108. [CrossRef]

381. Ferraro, D.A.; Gaborit, N.; Maron, R.; Cohen-Dvashi, H.; Porat, Z.; Pareja, F.; Lavi, S.; Lindzen, M.; Ben-Chetrit, N.; Sela, M.; et al. Inhibition of Triple-Negative Breast Cancer Models by Combinations of Antibodies to EGFR. Proc. Natl. Acad. Sci. USA 2013, 110, 1815-1820. [CrossRef]

382. Christensen, J.G.; Schreck, R.; Burrows, J.; Kuruganti, P.; Chan, E.; Le, P.; Chen, J.; Wang, X.; Ruslim, L.; Blake, R.; et al. A Selective Small Molecule Inhibitor of C-Met Kinase Inhibits c-Met-Dependent Phenotypes in Vitro and Exhibits Cytoreductive Antitumor Activity in Vivo. Cancer Res. 2003, 63, 7345-7355. [PubMed]

383. Bladt, F.; Faden, B.; Friese-Hamim, M.; Knuehl, C.; Wilm, C.; Fittschen, C.; Grädler, U.; Meyring, M.; Dorsch, D.; Jaehrling, F.; et al. EMD 1214063 and EMD 1204831 Constitute a New Class of Potent and Highly Selective C-Met Inhibitors. Am. Assoc. Cancer Res. 2013, 19, 2941-2951. [CrossRef]

384. Saura, C.; Roda, D.; Roselló, S.; Oliveira, M.; Macarulla, T.; Pérez-Fidalgo, J.A.; Morales-Barrera, R.; Sanchis-García, J.M.; Musib, L.; Budha, N.; et al. A First-in-Human Phase I Study of the ATP-Competitive AKT Inhibitor Ipatasertib Demonstrates Robust and Safe Targeting of AKT in Patients with Solid Tumors. Cancer Discov. 2017, 7, 102-113. [CrossRef]

385. Folkes, A.J.; Ahmadi, K.; Alderton, W.K.; Alix, S.; Baker, S.J.; Box, G.; Chuckowree, I.S.; Clarke, P.A.; Depledge, P.; Eccles, S.A.; et al. The Identification of 2-(1 H -Indazol-4-Yl)-6-(4-Methanesulfonyl-Piperazin-1-Ylmethyl)-4-Morpholin-4-Yl-Thieno[3,2d ]Pyrimidine (GDC-0941) as a Potent, Selective, Orally Bioavailable Inhibitor of Class I PI3 Kinase for the Treatment of Cancer. J. Med. Chem. 2008, 51, 5522-5532. [CrossRef]

386. Shor, B.; Zhang, W.-G.; Toral-Barza, L.; Lucas, J.; Abraham, R.T.; Gibbons, J.J.; Yu, K. A New Pharmacologic Action of CCI-779 Involves FKBP12-Independent Inhibition of MTOR Kinase Activity and Profound Repression of Global Protein Synthesis. Cancer Res. 2008, 68, 2934-2943. [CrossRef]

387. Madden, J.M.; Mueller, K.L.; Bollig-Fischer, A.; Stemmer, P.; Mattingly, R.R.; Boerner, J.L. Abrogating Phosphorylation of EIF4B Is Required for EGFR and MTOR Inhibitor Synergy in Triple-Negative Breast Cancer. Breast Cancer Res. Treat. 2014, 147, 283-293. [CrossRef]

388. French, K.J.; Schrecengost, R.S.; Lee, B.D.; Zhuang, Y.; Smith, S.N.; Eberly, J.L.; Yun, J.K.; Smith, C.D. Discovery and Evaluation of Inhibitors of Human Sphingosine Kinase. Cancer Res. 2003, 63, 5962-5969.

389. Martin, J.L.; de Silva, H.C.; Lin, M.Z.; Scott, C.D.; Baxter, R.C. Inhibition of Insulin-like Growth Factor-Binding Protein-3 Signaling through Sphingosine Kinase-1 Sensitizes Triple-Negative Breast Cancer Cells to EGF Receptor Blockade. Mol. Cancer 2014, 13, 316-328. [CrossRef] [PubMed]

390. Wang, X.; Le, P.; Liang, C.; Chan, J.; Kiewlich, D.; Miller, T.; Harris, D.; Sun, L.; Rice, A.; Vasile, S.; et al. Potent and Selective Inhibitors of the Met [Hepatocyte Growthfactor/Scatter Factor (HGF/SF) Receptor] Tyrosine Kinaseblock HGF/SF-Induced Tumor Cell Growthand Invasion. Mol. Cancer 2003, 2, 1085-1092. [CrossRef] [PubMed]

391. Huynh, H.; Soo, K.C.; Chow, P.K.H.; Tran, E. Targeted Inhibition of the Extracellular Signal-Regulated Kinase Kinase Pathway with AZD6244 (ARRY-142886) in the Treatment of Hepatocellular Carcinoma. Mol. Cancer 2007, 6, 138-146. [CrossRef]

392. Davis, M.I.; Hunt, J.P.; Herrgard, S.; Ciceri, P.; Wodicka, L.M.; Pallares, G.; Hocker, M.; Treiber, D.K.; Zarrinkar, P.P. Comprehensive Analysis of Kinase Inhibitor Selectivity. Nat. Biotechnol. 2011, 29, 1046-1051. [CrossRef] [PubMed]

393. Maiello, M.R.; D'Alessio, A.; Bevilacqua, S.; Gallo, M.; Normanno, N.; Luca, A.D. EGFR and MEK Blockade in Triple Negative Breast Cancer Cells. J. Cell Biochem. 2015, 116, 2778-2785. [CrossRef]

394. Randazzo, P.A.; Yang, Y.C.; Rulka, C.; Kahn, R.A. Activation of ADP-Ribosylation Factor by Golgi Membranes. Evidence for a Brefeldin A- and Protease-Sensitive Activating Factor on Golgi Membranes. J. Biol. Chem. 1993, 268, 9555-9563. [CrossRef]

395. Donaldson, J.G.; Finazzi, D.; Klausner, R.D. Brefeldin A Inhibits Golgi Membrane-Catalysed Exchange of Guanine Nucleotide onto ARF Protein. Nature 1992, 360, 350-352. [CrossRef] [PubMed]

396. Heinrich, M.C.; Griffith, D.J.; Druker, B.J.; Wait, C.L.; Ott, K.A.; Zigler, A.J. Inhibition of C-Kit Receptor Tyrosine Kinase Activity by STI 571, a Selective Tyrosine Kinase Inhibitor. Blood 2000, 96, 925-932. [CrossRef]

397. Wang, Y.-L.; Overstreet, A.-M.; Chen, M.-S.; Wang, J.; Zhao, H.-J.; Ho, P.-C.; Smith, M.; Wang, S.-C. Combined Inhibition of EGFR and C-ABL Suppresses the Growth of Triple-Negative Breast Cancer Growth through Inhibition of HOTAIR. Oncotarget 2015, 6, 11150-11161. [CrossRef]

398. Wang, X.; Song, H.; Yu, Q.; Liu, Q.; Wang, L.; Liu, Z.; Yu, Z. Ad-P53 Enhances the Sensitivity of Triple-Negative Breast Cancer MDA-MB-468 Cells to the EGFR Inhibitor Gefitinib. Oncol. Rep. 2015, 33, 526-532. [CrossRef] [PubMed]

399. Engstrom, L.D.; Aranda, R.; Lee, M.; Tovar, E.A.; Essenburg, C.J.; Madaj, Z.; Chiang, H.; Briere, D.; Hallin, J.; Lopez-Casas, P.P.; et al. Glesatinib Exhibits Antitumor Activity in Lung Cancer Models and Patients Harboring MET Exon 14 Mutations and Overcomes Mutation-Mediated Resistance to Type I MET Inhibitors in Nonclinical Models. Clin. Cancer Res. 2017, 23, 6661-6672. [CrossRef] 
400. Linklater, E.S.; Tovar, E.A.; Essenburg, C.J.; Turner, L.; Madaj, Z.; Winn, M.E.; Melnik, M.K.; Korkaya, H.; Maroun, C.R.; Christensen, J.G.; et al. Targeting MET and EGFR Crosstalk Signaling in Triple-Negative Breast Cancers. Oncotarget 2016, 7, 69903-69915. [CrossRef] [PubMed]

401. Zou, H.Y.; Li, Q.; Lee, J.H.; Arango, M.E.; McDonnell, S.R.; Yamazaki, S.; Koudriakova, T.B.; Alton, G.; Cui, J.J.; Kung, P.P.; et al. An Orally Available Small-Molecule Inhibitor of c-Met, PF-2341066, Exhibits Cytoreductive Antitumor Efficacy through Antiproliferative and Antiangiogenic Mechanisms. Cancer Res. 2007, 67, 4408-4417. [CrossRef]

402. Zou, H.Y.; Li, Q.; Engstrom, L.D.; West, M.; Appleman, V.; Wong, K.A.; McTigue, M.; Deng, Y.-L.; Liu, W.; Brooun, A.; et al. PF-06463922 Is a Potent and Selective next-Generation ROS1/ALK Inhibitor Capable of Blocking Crizotinib-Resistant ROS1 Mutations. Proc. Natl. Acad. Sci. USA 2015, 112, 3493-3498. [CrossRef]

403. Guerrab, A.E.; Bamdad, M.; Kwiatkowski, F.; Bignon, Y.-J.; Penault-Llorca, F.; Aubel, C. Anti-EGFR Monoclonal Antibodies and EGFR Tyrosine Kinase Inhibitors as Combination Therapy for Triple-Negative Breast Cancer. Oncotarget 2016, 5, 73618-73637. [CrossRef] [PubMed]

404. Doe, C.; Bentley, R.; Behm, D.J.; Lafferty, R.; Stavenger, R.; Jung, D.; Bamford, M.; Panchal, T.; Grygielko, E.; Wright, L.L.; et al. Novel Rho Kinase Inhibitors with Anti-Inflammatory and Vasodilatory Activities. J. Pharm. Exp. 2007, 320, 89-98. [CrossRef]

405. Iskit, S.; Lieftink, C.; Halonen, P.; Shahrabi, A.; Possik, P.A.; Beijersbergen, R.L.; Peeper, D.S. Integrated in Vivo Genetic and Pharmacologic Screening Identifies Co-Inhibition of EGRF and ROCK as a Potential Treatment Regimen for Triple-Negative Breast Cancer. Oncotarget 2016, 7, 42859-42872. [CrossRef]

406. Tanei, T.; Choi, D.S.; Rodriguez, A.A.; Liang, D.H.; Dobrolecki, L.; Ghosh, M.; Landis, M.D.; Chang, J.C. Antitumor Activity of Cetuximab in Combination with Ixabepilone on Triple Negative Breast Cancer Stem Cells. Breast Cancer Res. 2016, 18, 6. [CrossRef]

407. Guerrab, A.E.; Bamdad, M.; Bignon, Y.; Penault-Llorca, F.; Aubel, C. Anti-EGFR Monoclonal Antibodies Enhance Sensitivity to DNA-damaging Agents in BRCA1-mutated and PTEN-wild-type Triple-negative Breast Cancer Cells. Mol. Carcinog. 2017, 56, 1383-1394. [CrossRef]

408. Zhang, T.; Inesta-Vaquera, F.; Niepel, M.; Zhang, J.; Ficarro, S.B.; Machleidt, T.; Xie, T.; Marto, J.A.; Kim, N.; Sim, T.; et al. Discovery of Potent and Selective Covalent Inhibitors of JNK. Chem. Biol. 2012, 19, 140-154. [CrossRef] [PubMed]

409. Slack-Davis, J.K.; Martin, K.H.; Tilghman, R.W.; Iwanicki, M.; Ung, E.J.; Autry, C.; Luzzio, M.J.; Cooper, B.; Kath, J.C.; Roberts, W.G.; et al. Cellular Characterization of a Novel Focal Adhesion Kinase Inhibitor. J. Biol. Chem. 2007, 282, 14845-14852. [CrossRef] [PubMed]

410. Mitra, S.K.; Hanson, D.A.; Schlaepfer, D.D. Focal Adhesion Kinase: In Command and Control of Cell Motility. Nat. Rev. Mol. Cell Bio. 2005, 6, 56-68. [CrossRef] [PubMed]

411. Han, S.; Mistry, A.; Chang, J.S.; Cunningham, D.; Griffor, M.; Bonnette, P.C.; Wang, H.; Chrunyk, B.A.; Aspnes, G.E.; Walker, D.P.; et al. Structural Characterization of Proline-Rich Tyrosine Kinase 2 (PYK2) Reveals a Unique (DFG-out) Conformation and Enables Inhibitor Design. J. Biol. Chem. 2009, 284, 13193-13201. [CrossRef]

412. Rolin, J.; Sand, K.L.; Knudsen, E.; Maghazachi, A.A. FTY720 and SEW2871 Reverse the Inhibitory Effect of S1P on Natural Killer Cell Mediated Lysis of K562 Tumor Cells and Dendritic Cells but Not on Cytokine Release. Cancer Immunol. Immunother. 2010, 59, 575-586. [CrossRef]

413. Martin, J.L.; Julovi, S.M.; Lin, M.Z.; de Silva, H.C.; Boyle, F.M.; Baxter, R.C. Inhibition of Basal-like Breast Cancer Growth by FTY720 in Combination with Epidermal Growth Factor Receptor Kinase Blockade. Breast Cancer Res. 2017, 19, 90. [CrossRef]

414. Miller, S.; Tavshanjian, B.; Oleksy, A.; Perisic, O.; Houseman, B.T.; Shokat, K.M.; Williams, R.L. Shaping Development of Autophagy Inhibitors with the Structure of the Lipid Kinase Vps34. Science 2010, 327, 1638-1642. [CrossRef] [PubMed]

415. Liu, Z.; He, K.; Ma, Q.; Yu, Q.; Liu, C.; Ndege, I.; Wang, X.; Yu, Z. Autophagy Inhibitor Facilitates Gefitinib Sensitivity in Vitro and in Vivo by Activating Mitochondrial Apoptosis in Triple Negative Breast Cancer. PLoS ONE 2017, 12, e0177694. [CrossRef] [PubMed]

416. Al-Fifi, Z.I.A.; Marshall, S.L.; Hyde, D.; Anstee, J.H.; Bowler, K. Characterization of ATPases of Apical Membrane Fractions from Locusta Migratoria Malpighian Tubules. Insect Biochem. Mol. Biol. 1998, 28, 201-211. [CrossRef]

417. Bao, B.; Mitrea, C.; Wijesinghe, P.; Marchetti, L.; Girsch, E.; Farr, R.L.; Boerner, J.L.; Mohammad, R.; Dyson, G.; Terlecky, S.R.; et al. Treating Triple Negative Breast Cancer Cells with Erlotinib plus a Select Antioxidant Overcomes Drug Resistance by Targeting Cancer Cell Heterogeneity. Sci. Rep. 2017, 7, 44125. [CrossRef]

418. Colvin, T.A.; Gabai, V.L.; Gong, J.; Calderwood, S.K.; Li, H.; Gummuluru, S.; Matchuk, O.N.; Smirnova, S.G.; Orlova, N.V.; Zamulaeva, I.A.; et al. Hsp70-Bag3 Interactions Regulate Cancer-Related Signaling Networks. Cancer Res. 2014, 74, 4731-4740. [CrossRef]

419. Shields, S.; Conroy, E.; O'Grady, T.; McGoldrick, A.; Connor, K.; Ward, M.P.; Useckaite, Z.; Dempsey, E.; Reilly, R.; Fan, Y.; et al. BAG3 Promotes Tumour Cell Proliferation by Regulating EGFR Signal Transduction Pathways in Triple Negative Breast Cancer. Oncotarget 2018, 9, 15673-15690. [CrossRef]

420. Yap, T.A.; Yan, L.; Patnaik, A.; Fearen, I.; Olmos, D.; Papadopoulos, K.; Baird, R.D.; Delgado, L.; Taylor, A.; Lupinacci, L.; et al. First-in-Man Clinical Trial of the Oral Pan-AKT Inhibitor MK-2206 in Patients with Advanced Solid Tumors. J. Clin. Oncol. 2011, 29, 4688-4695. [CrossRef]

421. You, K.S.; Yi, Y.W.; Kwak, S.-J.; Seong, Y.-S. Inhibition of RPTOR Overcomes Resistance to EGFR Inhibition in Triple-Negative Breast Cancer Cells. Int. J. Oncol. 2018, 52, 828-840. [CrossRef] 
422. Qian, F.; Engst, S.; Yamaguchi, K.; Yu, P.; Won, K.-A.; Mock, L.; Lou, T.; Tan, J.; Li, C.; Tam, D.; et al. Inhibition of Tumor Cell Growth, Invasion, and Metastasis by EXEL-2880 (XL880, GSK1363089), a Novel Inhibitor of HGF and VEGF Receptor Tyrosine Kinases. Cancer Res. 2009, 69, 8009-8016. [CrossRef] [PubMed]

423. Simiczyjew, A.; Dratkiewicz, E.; Troys, M.V.; Ampe, C.; Styczeń, I.; Nowak, D. Combination of EGFR Inhibitor Lapatinib and MET Inhibitor Foretinib Inhibits Migration of Triple Negative Breast Cancer Cell Lines. Cancers 2018, 10, 335. [CrossRef]

424. Shin, S.-Y.; Müller, A.-K.; Verma, N.; Lev, S.; Nguyen, L.K. Systems Modelling of the EGFR-PYK2-c-Met Interaction Network Predicts and Prioritizes Synergistic Drug Combinations for Triple-Negative Breast Cancer. PLoS Comput. Biol. 2018, 14, e1006192. [CrossRef]

425. Montagnoli, A.; Valsasina, B.; Croci, V.; Menichincheri, M.; Rainoldi, S.; Marchesi, V.; Tibolla, M.; Tenca, P.; Brotherton, D.; Albanese, C.; et al. A Cdc7 Kinase Inhibitor Restricts Initiation of DNA Replication and Has Antitumor Activity. Nat. Chem. Biol. 2008, 4, 357-365. [CrossRef]

426. Fleisher, B.; Mody, H.; Werkman, C.; Ait-Oudhia, S. Chloroquine Sensitizes MDA-MB-231 Cells to Osimertinib through Autophagy-Apoptosis Crosstalk Pathway. Breast Cancer Targets 2019, 11, 231-241. [CrossRef] [PubMed]

427. Fry, D.W.; Harvey, P.J.; Keller, P.R.; Elliott, W.L.; Meade, M.; Trachet, E.; Albassam, M.; Zheng, X.; Leopold, W.R.; Pryer, N.K.; et al. Specific Inhibition of Cyclin-Dependent Kinase 4/6 by PD 0332991 and Associated Antitumor Activity in Human Tumor Xenografts. Mol. Cancer 2004, 3, 1427-1437.

428. Foidart, P.; Yip, C.; Radermacher, J.; Lienard, M.; Blacher, S.; Montero-Ruiz, L.; Maquoi, E.; Montaudon, E.; Chateau-Joubert, S.; Collignon, J.; et al. Expression of MT4-MMP, EGFR and RB in Triple Negative Breast Cancer Strongly Sensitizes Tumors to Erlotinib and Palbociclib Combination Therapy. Clin. Cancer Res. 2019, 25, 1838-1850. [CrossRef]

429. Sedrani, R.; Cottens, S.; Kallen, J.; Schuler, W. Chemical Modification of Rapamycin: The Discovery of SDZ RAD. Transplant. P 1998, 30, 2192-2194. [CrossRef]

430. He, J.; McLaughlin, R.P.; van der Noord, V.; Foekens, J.A.; Martens, J.W.M.; van Westen, G.; Zhang, Y.; van de Water, B. MultiTargeted Kinase Inhibition Alleviates MTOR Inhibitor Resistance in Triple-Negative Breast Cancer. Breast Cancer Res. Treat. 2019, 178, 263-274. [CrossRef]

431. Hanke, J.H.; Gardner, J.P.; Dow, R.L.; Changelian, P.S.; Brissette, W.H.; Weringer, E.J.; Pollok, B.A.; Connelly, P.A. Discovery of a Novel, Potent, and Src Family-Selective Tyrosine Kinase Inhibitor Study of Lck- and Fynt-Dependent T Cell Activation. J. Biol. Chem. 1996, 271, 695-701. [CrossRef]

432. Karni, R.; Mizrachi, S.; Reiss-Sklan, E.; Gazit, A.; Livnah, O.; Levitzki, A. The Pp60c-Src Inhibitor PP1 Is Non-competitive against ATP. Febs Lett. 2003, 537, 47-52. [CrossRef]

433. Tse, C.; Shoemaker, A.R.; Adickes, J.; Anderson, M.G.; Chen, J.; Jin, S.; Johnson, E.F.; Marsh, K.C.; Mitten, M.J.; Nimmer, P.; et al. ABT-263: A Potent and Orally Bioavailable Bcl-2 Family Inhibitor. Cancer Res. 2008, 68, 3421-3428. [CrossRef] [PubMed]

434. Kotschy, A.; Szlavik, Z.; Murray, J.; Davidson, J.; Maragno, A.L.; Toumelin-Braizat, G.L.; Chanrion, M.; Kelly, G.L.; Gong, J.-N.; Moujalled, D.M.; et al. The MCL1 Inhibitor S63845 Is Tolerable and Effective in Diverse Cancer Models. Nature 2016, 538, 477-482. [CrossRef]

435. Shen, Y.; Rehman, F.L.; Feng, Y.; Boshuizen, J.; Bajrami, I.; Elliott, R.; Wang, B.; Lord, C.J.; Post, L.E.; Ashworth, A. BMN 673, a Novel and Highly Potent PARP1/2 Inhibitor for the Treatment of Human Cancers with DNA Repair Deficiency. Clin. Cancer Res. 2013, 19, 5003-5015. [CrossRef] [PubMed]

436. Chu, Y.-Y.; Yam, C.; Chen, M.-K.; Chan, L.-C.; Xiao, M.; Wei, Y.-K.; Yamaguchi, H.; Lee, P.-C.; Han, Y.; Nie, L.; et al. Blocking C-Met and EGFR Reverses Acquired Resistance of PARP Inhibitors in Triple-Negative Breast Cancer. Am. J. Cancer Res. 2020, 10, 648-661. [PubMed]

437. Kwiatkowski, N.; Zhang, T.; Rahl, P.B.; Abraham, B.J.; Reddy, J.; Ficarro, S.B.; Dastur, A.; Amzallag, A.; Ramaswamy, S.; Tesar, B.; et al. Targeting Transcription Regulation in Cancer with a Covalent CDK7 Inhibitor. Nature 2014, 511, 616-620. [CrossRef]

438. McDermott, M.S.J.; Sharko, A.C.; Munie, J.; Kassler, S.; Melendez, T.; Lim, C.; Broude, E.V. CDK7 Inhibition Is Effective in All the Subtypes of Breast Cancer: Determinants of Response and Synergy with EGFR Inhibition. Cells 2020, 9, 638. [CrossRef] [PubMed]

439. Juliá, E.P.; Mordoh, J.; Levy, E.M. Cetuximab and IL-15 Promote NK and Dendritic Cell Activation In Vitro in Triple Negative Breast Cancer. Cells 2020, 9, 1573. [CrossRef] [PubMed]

440. McKnight, B.N.; Kim, S.; Boerner, J.L.; Viola, N.T. Cetuximab PET Delineated Changes in Cellular Distribution of EGFR upon Dasatinib Treatment in Triple Negative Breast Cancer. Breast Cancer Res. 2020, 22, 37. [CrossRef]

441. Canonici, A.; Browne, A.L.; Ibrahim, M.F.K.; Fanning, K.P.; Roche, S.; Conlon, N.T.; O’Neill, F.; Meiller, J.; Cremona, M.; Morgan, C.; et al. Combined Targeting EGFR and SRC as a Potential Novel Therapeutic Approach for the Treatment of Triple Negative Breast Cancer. Adv. Med. Oncol. 2020, 12, 1758835919897546. [CrossRef]

442. Guerrab, A.E.; Bamdad, M.; Bignon, Y.-J.; Penault-Llorca, F.; Aubel, C. Co-Targeting EGFR and MTOR with Gefitinib and Everolimus in Triple-Negative Breast Cancer Cells. Sci. Rep. 2020, 10, 6367. [CrossRef]

443. Yamaguchi, T.; Kakefuda, R.; Tajima, N.; Sowa, Y.; Sakai, T. Antitumor Activities of JTP-74057 (GSK1120212), a Novel MEK1/2 Inhibitor, on Colorectal Cancer Cell Lines in Vitro and in Vivo. Int. J. Oncol. 2011, 39, 23-31. [CrossRef]

444. Ahmad, S.; He, Q.; Williams, K.P.; Scott, J.E. Identification of a Triple Drug Combination That Is Synergistically Cytotoxic for Triple-Negative Breast Cancer Cells Using a Novel Combination Discovery Approach. SLAS Discov. 2020, 25, 923-938. [CrossRef] [PubMed] 
445. Rau, A.; Lieb, W.S.; Seifert, O.; Honer, J.; Birnstock, D.; Richter, F.; Aschmoneit, N.; Olayioye, M.A.; Kontermann, R.E. Inhibition of Tumor Cell Growth and Cancer Stem Cell Expansion by a Bispecific Antibody Targeting EGFR and HER3. Mol. Cancer 2020, 19, 1474-1485. [CrossRef]

446. Reddy, T.P.; Choi, D.S.; Anselme, A.C.; Qian, W.; Chen, W.; Lantto, J.; Horak, I.D.; Kragh, M.; Chang, J.C.; Rosato, R.R. Simultaneous Targeting of HER Family Pro-Survival Signaling with Pan-HER Antibody Mixture Is Highly Effective in TNBC: A Preclinical Trial with PDXs. Breast Cancer Res. 2020, 22, 48. [CrossRef]

447. Caratelli, S.; Arriga, R.; Sconocchia, T.; Ottaviani, A.; Lanzilli, G.; Pastore, D.; Cenciarelli, C.; Venditti, A.; Principe, M.I.D.; Lauro, D.; et al. In Vitro Elimination of Epidermal Growth Factor Receptor-overexpressing Cancer Cells by CD32A-chimeric Receptor T Cells in Combination with Cetuximab or Panitumumab. Int. J. Cancer 2020, 146, 236-247. [CrossRef] [PubMed]

448. Nakahara, T.; Takeuchi, M.; Kinoyama, I.; Minematsu, T.; Shirasuna, K.; Matsuhisa, A.; Kita, A.; Tominaga, F.; Yamanaka, K.; Kudoh, M.; et al. YM155, a Novel Small-Molecule Survivin Suppressant, Induces Regression of Established Human HormoneRefractory Prostate Tumor Xenografts. Cancer Res. 2007, 67, 8014-8021. [CrossRef] [PubMed]

449. Turner, T.H.; Alzubi, M.A.; Harrell, J.C. Identification of Synergistic Drug Combinations Using Breast Cancer Patient-Derived Xenografts. Sci. Rep. 2020, 10, 1493. [CrossRef] [PubMed]

450. Yin, L.; Qi, X.-W.; Liu, X.-Z.; Yang, Z.-Y.; Cai, R.-L.; Cui, H.-J.; Chen, L.; Yu, S.-C. Icaritin Enhances the Efficacy of Cetuximab against Triple-Negative Breast Cancer Cells. Oncol. Lett. 2020, 19, 3950-3958. [CrossRef]

451. Zecchin, D.; Moore, C.; Michailidis, F.; Horswell, S.; Rana, S.; Howell, M.; Downward, J. Combined Targeting of G Protein-coupled Receptor and EGF Receptor Signaling Overcomes Resistance to PI3K Pathway Inhibitors in PTEN-null Triple Negative Breast Cancer. EMBO Mol. Med. 2020, 12, e11987. [CrossRef]

452. Barlaam, B.; Cosulich, S.; Degorce, S.; Fitzek, M.; Green, S.; Hancox, U.; der Brempt, C.L.; Lohmann, J.-J.; Maudet, M.; Morgentin, R.; et al. Discovery of (R)-8-(1-(3,5-Difluorophenylamino)Ethyl)-N,N-dimethyl-2-Morpholino-4-Oxo-4H-chromene-6Carboxamide (AZD8186): A Potent and Selective Inhibitor of PI3K $\beta$ and PI3K $\delta$ for the Treatment of PTEN-Deficient Cancers. J. Med. Chem. 2015, 58, 943-962. [CrossRef]

453. Xu, W.; Song, C.; Wang, X.; Li, Y.; Bai, X.; Liang, X.; Wu, J.; Liu, J. Downregulation of MiR-155-5p Enhances the Anti-Tumor Effect of Cetuximab on Triple-Negative Breast Cancer Cells via Inducing Cell Apoptosis and Pyroptosis. Aging 2021, 228-240. [CrossRef] [PubMed]

454. Grimshaw, K.M.; Hunter, L.-J.K.; Yap, T.A.; Heaton, S.P.; Walton, M.I.; Woodhead, S.J.; Fazal, L.; Reule, M.; Davies, T.G.; Seavers, L.C.; et al. AT7867 Is a Potent and Oral Inhibitor of AKT and P70 S6 Kinase That Induces Pharmacodynamic Changes and Inhibits Human Tumor Xenograft Growth. Mol. Cancer 2010, 9, 1100-1110. [CrossRef]

455. Barrett, S.D.; Bridges, A.J.; Dudley, D.T.; Saltiel, A.R.; Fergus, J.H.; Flamme, C.M.; Delaney, A.M.; Kaufman, M.; LePage, S.; Leopold, W.R.; et al. The Discovery of the Benzhydroxamate MEK Inhibitors CI-1040 and PD 0325901. Bioorg. Med. Chem. Lett. 2008, 18, 6501-6504. [CrossRef] [PubMed]

456. Koepke, J.I.; Nakrieko, K.; Wood, C.S.; Boucher, K.K.; Terlecky, L.J.; Walton, P.A.; Terlecky, S.R. Restoration of Peroxisomal Catalase Import in a Model of Human Cellular Aging. Traffic 2007, 8, 1590-1600. [CrossRef] [PubMed]

457. Carles, F.; Bourg, S.; Meyer, C.; Bonnet, P. PKIDB: A Curated, Annotated and Updated Database of Protein Kinase Inhibitors in Clinical Trials. Molecules 2018, 23, 908. [CrossRef] [PubMed]

458. Bournez, C.; Carles, F.; Peyrat, G.; Aci-Sèche, S.; Bourg, S.; Meyer, C.; Bonnet, P. Comparative Assessment of Protein Kinase Inhibitors in Public Databases and in PKIDB. Molecules 2020, 25, 3226. [CrossRef] [PubMed]

459. Anido, J.; Matar, P.; Albanell, J.; Guzmán, M.; Rojo, F.; Arribas, J.; Averbuch, S.; Baselga, J. ZD1839, a Specific Epidermal Growth Factor Receptor (EGFR) Tyrosine Kinase Inhibitor, Induces the Formation of Inactive EGFR/HER2 and EGFR/HER3 Heterodimers and Prevents Heregulin Signaling in HER2-Overexpressing Breast Cancer Cells. Clin. Cancer Res. Off. J. Am. Assoc. Cancer Res. 2003, 9, 1274-1283.

460. Senichkin, V.V.; Streletskaia, A.Y.; Gorbunova, A.S.; Zhivotovsky, B.; Kopeina, G.S. Saga of Mcl-1: Regulation from Transcription to Degradation. Cell Death Differ. 2020, 27, 405-419. [CrossRef]

461. Deng, M.; Wang, J.; Chen, Y.; Zhang, L.; Liu, D. Combination of SF1126 and Gefitinib Induces Apoptosis of Triple-Negative Breast Cancer Cells through the PI3K/AKT-MTOR Pathway. Anti Cancer Drug 2015, 26, 422-427. [CrossRef]

462. De, P.; Dey, N.; Terakedis, B.; Bergsagel, P.L.; Li, Z.H.; Mahadevan, D.; Garlich, J.R.; Trudel, S.; Makale, M.T.; Durden, D.L. An Integrin-Targeted, Pan-Isoform, Phosphoinositide-3 Kinase Inhibitor, SF1126, Has Activity against Multiple Myeloma in Vivo. Cancer Chemoth. Pharm. 2013, 71, 867-881. [CrossRef]

463. Heitman, J.; Movva, N.; Hall, M. Targets for Cell Cycle Arrest by the Immunosuppressant Rapamycin in Yeast. Science 1991, 253, 905-909. [CrossRef] [PubMed]

464. Kim, D.-H.; Sarbassov, D.D.; Ali, S.M.; King, J.E.; Latek, R.R.; Erdjument-Bromage, H.; Tempst, P.; Sabatini, D.M. MTOR Interacts with Raptor to Form a Nutrient-Sensitive Complex That Signals to the Cell Growth Machinery. Cell 2002, 110, 163-175. [CrossRef]

465. Polunovsky, V.A.; Rosenwald, I.B.; Tan, A.T.; White, J.; Chiang, L.; Sonenberg, N.; Bitterman, P.B. Translational Control of Programmed Cell Death: Eukaryotic Translation Initiation Factor 4E Blocks Apoptosis in Growth-Factor-Restricted Fibroblasts with Physiologically Expressed or Deregulated Myc. Mol. Cell Biol. 1996, 16, 6573-6581. [CrossRef] [PubMed]

466. Wendel, H.-G.; Silva, R.L.A.; Malina, A.; Mills, J.R.; Zhu, H.; Ueda, T.; Watanabe-Fukunaga, R.; Fukunaga, R.; Teruya-Feldstein, J.; Pelletier, J.; et al. Dissecting EIF4E Action in Tumorigenesis. Gene Dev. 2007, 21, 3232-3237. [CrossRef] [PubMed] 
467. Meng, L.; Zheng, X.S. Toward Rapamycin Analog (Rapalog)-Based Precision Cancer Therapy. Acta Pharm. Sin. 2015, 36, 1163-1169. [CrossRef]

468. Salmond, R.J.; Emery, J.; Okkenhaug, K.; Zamoyska, R. MAPK, Phosphatidylinositol 3-Kinase, and Mammalian Target of Rapamycin Pathways Converge at the Level of Ribosomal Protein S6 Phosphorylation to Control Metabolic Signaling in CD8 T Cells. J. Immunol. 2009, 183, 7388-7397. [CrossRef]

469. Shahbazian, D.; Roux, P.P.; Mieulet, V.; Cohen, M.S.; Raught, B.; Taunton, J.; Hershey, J.W.; Blenis, J.; Pende, M.; Sonenberg, N. The MTOR/PI3K and MAPK Pathways Converge on EIF4B to Control Its Phosphorylation and Activity. EMBO J. 2006, 25, $2781-2791$. [CrossRef] [PubMed]

470. Traxler, P.; Allegrini, P.R.; Brandt, R.; Brueggen, J.; Cozens, R.; Fabbro, D.; Grosios, K.; Lane, H.A.; McSheehy, P.; Mestan, J.; et al. AEE788 A Dual Family Epidermal Growth Factor Receptor/ErbB2 and Vascular Endothelial Growth Factor Receptor Tyrosine Kinase Inhibitor with Antitumor and Antiangiogenic Activity. Cancer Res. 2004, 64, 4931-4941. [CrossRef]

471. Keraite, I.; Alvarez-Garcia, V.; Garcia-Murillas, I.; Beaney, M.; Turner, N.C.; Bartos, C.; Oikonomidou, O.; Kersaudy-Kerhoas, M.; Leslie, N.R. PIK3CA Mutation Enrichment and Quantitation from Blood and Tissue. Sci. Rep. 2020, 10, 17082. [CrossRef] [PubMed]

472. Fayette, J.; Wirth, L.; Oprean, C.; Udrea, A.; Jimeno, A.; Rischin, D.; Nutting, C.; Harari, P.M.; Csoszi, T.; Cernea, D.; et al. Randomized Phase II Study of Duligotuzumab (MEHD7945A) vs. Cetuximab in Squamous Cell Carcinoma of the Head and Neck (MEHGAN Study). Front. Oncol. 2016, 6, 232. [CrossRef]

473. Hill, A.G.; Findlay, M.; Burge, M.; Jackson, C.; Alfonso, P.G.; Samuel, L.; Ganju, V.; Karthaus, M.; Amatu, A.; Jeffery, M.; et al. Phase II Study of the Dual EGFR/HER3 Inhibitor Duligotuzumab (MEHD7945A) vs. Cetuximab in Combination with FOLFIRI in RAS Wild-Type Metastatic Colorectal Cancer. Clin. Cancer Res. 2018, 24, 2276-2284. [CrossRef]

474. Chandarlapaty, S.; Sawai, A.; Scaltriti, M.; Rodrik-Outmezguine, V.; Grbovic-Huezo, O.; Serra, V.; Majumder, P.K.; Baselga, J.; Rosen, N. AKT Inhibition Relieves Feedback Suppression of Receptor Tyrosine Kinase Expression and Activity. Cancer Cell 2011, 19, 58-71. [CrossRef] [PubMed]

475. Serra, V.; Scaltriti, M.; Prudkin, L.; Eichhorn, P.J.A.; Ibrahim, Y.H.; Chandarlapaty, S.; Markman, B.; Rodriguez, O.; Guzman, M.; Rodriguez, S.; et al. PI3K Inhibition Results in Enhanced HER Signaling and Acquired ERK Dependency in HER2-Overexpressing Breast Cancer. Oncogene 2011, 30, 2547-2557. [CrossRef]

476. Rodrik-Outmezguine, V.S.; Chandarlapaty, S.; Pagano, N.C.; Poulikakos, P.I.; Scaltriti, M.; Moskatel, E.; Baselga, J.; Guichard, S.; Rosen, N. MTOR Kinase Inhibition Causes Feedback-Dependent Biphasic Regulation of AKT Signaling. Cancer Discov. 2011, 1, 248-259. [CrossRef]

477. Kane, R.C.; Farrell, A.T.; Sridhara, R.; Pazdur, R. United States Food and Drug Administration Approval Summary: Bortezomib for the Treatment of Progressive Multiple Myeloma after One Prior Therapy. Clin. Cancer Res. 2006, 12, 2955-2960. [CrossRef] [PubMed]

478. Shahshahan, M.A.; Beckley, M.N.; Jazirehi, A.R. Potential Usage of Proteasome Inhibitor Bortezomib (Velcade, PS-341) in the Treatment of Metastatic Melanoma: Basic and Clinical Aspects. Am. J. Cancer Res. 2011, 1, 913-924. [PubMed]

479. Nakshatri, H.; Bhat-Nakshatri, P.; Martin, D.A.; Goulet, R.J.; Sledge, G.W. Constitutive Activation of NF-KappaB during Progression of Breast Cancer to Hormone-Independent Growth. Mol. Cell Biol. 1997, 17, 3629-3639. [CrossRef] [PubMed]

480. Yang, C.H.; Gonzalez-Angulo, A.M.; Reuben, J.M.; Booser, D.J.; Pusztai, L.; Krishnamurthy, S.; Esseltine, D.; Stec, J.; Broglio, K.R.; Islam, R.; et al. Bortezomib (VELCADE ${ }^{\circledR}$ ) in Metastatic Breast Cancer: Pharmacodynamics, Biological Effects, and Prediction of Clinical Benefits. Ann. Oncol. 2006, 17, 813-817. [CrossRef]

481. Duong, H.-Q.; You, K.; Oh, S.; Kwak, S.-J.; Seong, Y.-S. Silencing of NRF2 Reduces the Expression of ALDH1A1 and ALDH3A1 and Sensitizes to 5-FU in Pancreatic Cancer Cells. Antioxidants 2017, 6, 52. [CrossRef]

482. Malumbres, M.; Harlow, E.; Hunt, T.; Hunter, T.; Lahti, J.M.; Manning, G.; Morgan, D.O.; Tsai, L.-H.; Wolgemuth, D.J. CyclinDependent Kinases: A Family Portrait. Nat. Cell Biol. 2009, 11, 1275-1276. [CrossRef]

483. Roskoski, R. Cyclin-Dependent Protein Serine/Threonine Kinase Inhibitors as Anticancer Drugs. Pharm. Res. 2018, 139, 471-488. [CrossRef] [PubMed]

484. Cassandri, M.; Fioravanti, R.; Pomella, S.; Valente, S.; Rotili, D.; Baldo, G.D.; Angelis, B.D.; Rota, R.; Mai, A. CDK9 as a Valuable Target in Cancer: From Natural Compounds Inhibitors to Current Treatment in Pediatric Soft Tissue Sarcomas. Front. Pharm. 2020, 11, 1230. [CrossRef]

485. Chou, J.; Quigley, D.A.; Robinson, T.M.; Feng, F.Y.; Ashworth, A. Transcription-Associated Cyclin-Dependent Kinases as Targets and Biomarkers for Cancer Therapy. Cancer Discov. 2020, 10, 351-370. [CrossRef]

486. Giacinti, C.; Giordano, A. RB and Cell Cycle Progression. Oncogene 2006, 25, 5220-5227. [CrossRef]

487. Besson, A.; Dowdy, S.F.; Roberts, J.M. CDK Inhibitors: Cell Cycle Regulators and Beyond. Dev. Cell 2008, 14, 159-169. [CrossRef] [PubMed]

488. Paye, A.; Truong, A.; Yip, C.; Cimino, J.; Blacher, S.; Munaut, C.; Cataldo, D.; Foidart, J.M.; Maquoi, E.; Collignon, J.; et al. EGFR Activation and Signaling in Cancer Cells Are Enhanced by the Membrane-Bound Metalloprotease MT4-MMP. Cancer Res. 2014, 74, 6758-6770. [CrossRef]

489. Yip, C.; Foidart, P.; Somja, J.; Truong, A.; Lienard, M.; Feyereisen, E.; Schroeder, H.; Gofflot, S.; Donneau, A.-F.; Collignon, J.; et al. MT4-MMP and EGFR Expression Levels Are Key Biomarkers for Breast Cancer Patient Response to Chemotherapy and Erlotinib. Br. J. Cancer 2017, 116, 742-751. [CrossRef] 
490. Peng, J.; Zhu, Y.; Milton, J.T.; Price, D.H. Identification of Multiple Cyclin Subunits of Human P-TEFb. Gene Dev. 1998, 12, 755-762. [CrossRef] [PubMed]

491. Larochelle, S.; Amat, R.; Glover-Cutter, K.; Sansó, M.; Zhang, C.; Allen, J.J.; Shokat, K.M.; Bentley, D.L.; Fisher, R.P. CyclinDependent Kinase Control of the Initiation-to-Elongation Switch of RNA Polymerase II. Nat. Struct Mol. Biol. 2012, 19, $1108-1115$. [CrossRef] [PubMed]

492. Viladevall, L.; Amour, C.V.S.; Rosebrock, A.; Schneider, S.; Zhang, C.; Allen, J.J.; Shokat, K.M.; Schwer, B.; Leatherwood, J.K.; Fisher, R.P. TFIIH and P-TEFb Coordinate Transcription with Capping Enzyme Recruitment at Specific Genes in Fission Yeast. Mol. Cell 2009, 33, 738-751. [CrossRef]

493. Yang, Z.; Yik, J.H.N.; Chen, R.; He, N.; Jang, M.K.; Ozato, K.; Zhou, Q. Recruitment of P-TEFb for Stimulation of Transcriptional Elongation by the Bromodomain Protein Brd4. Mol. Cell 2005, 19, 535-545. [CrossRef]

494. Ahn, S.H.; Kim, M.; Buratowski, S. Phosphorylation of Serine 2 within the RNA Polymerase II C-Terminal Domain Couples Transcription and $3^{\prime}$ End Processing. Mol. Cell 2004, 13, 67-76. [CrossRef]

495. Li, B.; Chonghaile, T.N.; Fan, Y.; Madden, S.F.; Klinger, R.; O'Connor, A.E.; Walsh, L.; O'Hurley, G.; Udupi, G.M.; Joseph, J.; et al. Therapeutic Rationale to Target Highly Expressed CDK7 Conferring Poor Outcomes in Triple-Negative Breast Cancer. Cancer Res. 2017, 77, 3834-3845. [CrossRef] [PubMed]

496. Wang, Y.; Zhang, T.; Kwiatkowski, N.; Abraham, B.J.; Lee, T.I.; Xie, S.; Yuzugullu, H.; Von, T.; Li, H.; Lin, Z.; et al. CDK7-Dependent Transcriptional Addiction in Triple-Negative Breast Cancer. Cell 2015, 163, 174-186. [CrossRef]

497. Sun, B.; Mason, S.; Wilson, R.C.; Hazard, S.E.; Wang, Y.; Fang, R.; Wang, Q.; Yeh, E.S.; Yang, M.; Roberts, T.M.; et al. Inhibition of the Transcriptional Kinase CDK7 Overcomes Therapeutic Resistance in HER2-Positive Breast Cancers. Oncogene 2020, 39, 50-63. [CrossRef]

498. Labib, K.; Tercero, J.A.; Diffley, J.F.X. Uninterrupted MCM2-7 Function Required for DNA Replication Fork Progression. Science 2000, 288, 1643-1647. [CrossRef]

499. Benedict, B.; van Harn, T.; Dekker, M.; Hermsen, S.; Kucukosmanoglu, A.; Pieters, W.; Delzenne-Goette, E.; Dorsman, J.C.; Petermann, E.; Foijer, F.; et al. Loss of P53 Suppresses Replication-Stress-Induced DNA Breakage in G1/S Checkpoint Deficient Cells. Elife 2018, 7, e37868. [CrossRef] [PubMed]

500. Parsons, S.J.; Parsons, J.T. Src Family Kinases, Key Regulators of Signal Transduction. Oncogene 2004, 23, 7906-7909. [CrossRef]

501. Boggon, T.J.; Eck, M.J. Structure and Regulation of Src Family Kinases. Oncogene 2004, 23, 7918-7927. [CrossRef]

502. Lindauer, M.; Hochhaus, A. Small Molecules in Oncology. Recent Results Cancer Res. 2014, 201, 27-65. [CrossRef]

503. Kim, D.-J.; Yi, Y.-W.; Kim, J.H. In Situ Monitoring of Bindings between Dasatinib and Its Target Protein Kinases Using Magnetic Nanoparticles in Live Cells. J. Am Chem. Soc. 2008, 130, 16466-16467. [CrossRef] [PubMed]

504. Gupta, R.A.; Shah, N.; Wang, K.C.; Kim, J.; Horlings, H.M.; Wong, D.J.; Tsai, M.-C.; Hung, T.; Argani, P.; Rinn, J.L.; et al. Long Non-Coding RNA HOTAIR Reprograms Chromatin State to Promote Cancer Metastasis. Nature 2010, 464, 1071-1076. [CrossRef]

505. Wu, Y.; Zhang, L.; Zhang, L.; Wang, Y.; Li, H.; Ren, X.; Wei, F.; Yu, W.; Liu, T.; Wang, X.; et al. Long Non-Coding RNA HOTAIR Promotes Tumor Cell Invasion and Metastasis by Recruiting EZH2 and Repressing E-Cadherin in Oral Squamous Cell Carcinoma. Int. J. Oncol. 2015, 46, 2586-2594. [CrossRef] [PubMed]

506. Xia, M.; Yao, L.; Zhang, Q.; Wang, F.; Mei, H.; Guo, X.; Huang, W. Long Noncoding RNA HOTAIR Promotes Metastasis of Renal Cell Carcinoma by Up-Regulating Histone H3K27 Demethylase JMJD3. Oncotarget 2014, 5, 19795-19802. [CrossRef]

507. Yom, C.K.; Noh, D.-Y.; Kim, W.H.; Kim, H.S. Clinical Significance of High Focal Adhesion Kinase Gene Copy Number and Overexpression in Invasive Breast Cancer. Breast Cancer Res. Treat. 2011, 128, 647-655. [CrossRef]

508. Boyd, Z.S.; Wu, Q.J.; O’Brien, C.; Spoerke, J.; Savage, H.; Fielder, P.J.; Amler, L.; Yan, Y.; Lackner, M.R. Proteomic Analysis of Breast Cancer Molecular Subtypes and Biomarkers of Response to Targeted Kinase Inhibitors Using Reverse-Phase Protein Microarrays. Mol. Cancer 2008, 7, 3695-3706. [CrossRef]

509. Glenisson, M.; Vacher, S.; Callens, C.; Susini, A.; Cizeron-Clairac, G.; Scodan, R.L.; Meseure, D.; Lerebours, F.; Spyratos, F.; Lidereau, R.; et al. Identification of New Candidate Therapeutic Target Genes in Triple-Negative Breast Cancer. Genes Cancer 2012, 3, 63-70. [CrossRef]

510. Sun, J.; Zhang, D.; Bae, D.-H.; Sahni, S.; Jansson, P.; Zheng, Y.; Zhao, Q.; Yue, F.; Zheng, M.; Kovacevic, Z.; et al. Metastasis Suppressor, NDRG1, Mediates Its Activity through Signaling Pathways and Molecular Motors. Carcinogenesis 2013, 34, 1943-1954. [CrossRef]

511. Ono-Saito, N.; Niki, I.; Hidaka, H. H-Series Protein Kinase Inhibitors and Potential Clinical Applications. Pharm. Ther. 1999, 82, 123-131. [CrossRef]

512. Zhao, J.; Zhou, D.; Guo, J.; Ren, Z.; Zhou, L.; Wang, S.; Xu, B.; Wang, R. Effect of Fasudil Hydrochloride, a Protein Kinase Inhibitor, on Cerebral Vasospasm and Delayed Cerebral Ischemic Symptoms After Aneurysmal Subarachnoid Hemorrhage. Neurol. Med. Chir 2006, 46, 421-428. [CrossRef] [PubMed]

513. Sun, Y.; Lin, X.; Aske, J.C.; Ye, P.; Williams, C.; Abramovitz, M.; Leyland-Jones, B.R. Dasatinib Attenuates Overexpression of Src Signaling Induced by the Combination Treatment of Veliparib plus Carboplatin in Triple-Negative Breast Cancer. Cancer Chemoth. Pharm. 2019, 84, 1241-1256. [CrossRef] [PubMed]

514. Tryfonopoulos, D.; Walsh, S.; Collins, D.M.; Flanagan, L.; Quinn, C.; Corkery, B.; McDermott, E.W.; Evoy, D.; Pierce, A.; O'Donovan, N.; et al. Src: A Potential Target for the Treatment of Triple-Negative Breast Cancer. Ann. Oncol. 2011, 22, 2234-2240. [CrossRef] [PubMed] 
515. Adams, B.D.; Wali, V.B.; Cheng, C.J.; Inukai, S.; Booth, C.J.; Agarwal, S.; Rimm, D.L.; Győrffy, B.; Santarpia, L.; Pusztai, L.; et al. MiR-34a Silences c-SRC to Attenuate Tumor Growth in Triple-Negative Breast Cancer. Cancer Res. 2016, 76, 927-939. [CrossRef] [PubMed]

516. Qian, X.-L.; Zhang, J.; Li, P.-Z.; Lang, R.-G.; Li, W.-D.; Sun, H.; Liu, F.-F.; Guo, X.-J.; Gu, F.; Fu, L. Dasatinib Inhibits C-Src Phosphorylation and Prevents the Proliferation of Triple-Negative Breast Cancer (TNBC) Cells Which Overexpress SyndecanBinding Protein (SDCBP). PLoS ONE 2017, 12, e0171169. [CrossRef]

517. Lou, L.; Yu, Z.; Wang, Y.; Wang, S.; Zhao, Y. C-Src Inhibitor Selectively Inhibits Triple-negative Breast Cancer Overexpressed Vimentin in Vitro and in Vivo. Cancer Sci. 2018, 109, 1648-1659. [CrossRef]

518. Song, L.; Morris, M.; Bagui, T.; Lee, F.Y.; Jove, R.; Haura, E.B. Dasatinib (BMS-354825) Selectively Induces Apoptosis in Lung Cancer Cells Dependent on Epidermal Growth Factor Receptor Signaling for Survival. Cancer Res. 2006, 66, 5542-5548. [CrossRef]

519. Lin, Y.-C.; Wu, M.-H.; Wei, T.-T.; Chung, S.-H.; Chen, K.-F.; Cheng, A.-L.; Chen, C.-C. Degradation of Epidermal Growth Factor Receptor Mediates Dasatinib-Induced Apoptosis in Head and Neck Squamous Cell Carcinoma Cells. Neoplasia 2012, 14, 463-475. [CrossRef]

520. Duong, H.-Q.; Yi, Y.W.; Kang, H.J.; Bae, I.; Jang, Y.-J.; Kwak, S.-J.; Seong, Y.-S. Combination of Dasatinib and Gemcitabine Reduces the ALDH1A1 Expression and the Proliferation of Gemcitabine-Resistant Pancreatic Cancer MIA PaCa-2 Cells. Int. J. Oncol. 2014, 44, 2132-2138. [CrossRef] [PubMed]

521. Marcato, P.; Dean, C.A.; Giacomantonio, C.A.; Lee, P.W.K. Aldehyde Dehydrogenase: Its Role as a Cancer Stem Cell Marker Comes down to the Specific Isoform. Cell Cycle 2011, 10, 1378-1384. [CrossRef] [PubMed]

522. Pilié, P.G.; Gay, C.M.; Byers, L.A.; O'Connor, M.J.; Yap, T.A. PARP Inhibitors: Extending Benefit Beyond BRCA-Mutant Cancers. Clin. Cancer Res. 2019, 25, 3759-3771. [CrossRef] [PubMed]

523. Keung, M.Y.; Wu, Y.; Badar, F.; Vadgama, J.V. Response of Breast Cancer Cells to PARP Inhibitors Is Independent of BRCA Status. J. Clin. Med. 2020, 9, 940. [CrossRef]

524. Elstrodt, F.; Hollestelle, A.; Nagel, J.H.A.; Gorin, M.; Wasielewski, M.; van den Ouweland, A.; Merajver, S.D.; Ethier, S.P.; Schutte, M. BRCA1 Mutation Analysis of 41 Human Breast Cancer Cell Lines Reveals Three New Deleterious Mutants. Cancer Res. 2006, 66, 41-45. [CrossRef]

525. Kumaraswamy, E.; Wendt, K.L.; Augustine, L.A.; Stecklein, S.R.; Sibala, E.C.; Li, D.; Gunewardena, S.; Jensen, R.A. BRCA1 Regulation of Epidermal Growth Factor Receptor (EGFR) Expression in Human Breast Cancer Cells Involves MicroRNA-146a and Is Critical for Its Tumor Suppressor Function. Oncogene 2015, 34, 4333-4346. [CrossRef]

526. Yi, Y.W.; Park, J.-S.; Kwak, S.-J.; Seong, Y.-S. Co-Treatment with BEZ235 Enhances Sensitivity of BRCA1-Negative Breast Cancer Cells to Olaparib. Anticancer Res. 2015, 35, 3829-3838.

527. Reilly, E.B.; Phillips, A.C.; Buchanan, F.G.; Kingsbury, G.; Zhang, Y.; Meulbroek, J.A.; Cole, T.B.; DeVries, P.J.; Falls, H.D.; Beam, C.; et al. Characterization of ABT-806, a Humanized Tumor-Specific Anti-EGFR Monoclonal Antibody. Mol. Cancer 2015, 14, 1141-1151. [CrossRef]

528. Phillips, A.C.; Boghaert, E.R.; Vaidya, K.S.; Mitten, M.J.; Norvell, S.; Falls, H.D.; DeVries, P.J.; Cheng, D.; Meulbroek, J.A.; Buchanan, F.G.; et al. ABT-414, an Antibody-Drug Conjugate Targeting a Tumor-Selective EGFR Epitope. Mol. Cancer 2016, 15, 661-669. [CrossRef]

529. Anderson, M.G.; Falls, H.D.; Mitten, M.J.; Oleksijew, A.; Vaidya, K.S.; Boghaert, E.R.; Gao, W.; Palma, J.P.; Cao, D.; Chia, P.-L.; et al. Targeting Multiple EGFR-Expressing Tumors with a Highly Potent Tumor-Selective Antibody-Drug Conjugate. Mol. Cancer 2020, 19, 2117-2125. [CrossRef] [PubMed]

530. Guillermet-Guibert, J.; Davenne, L.; Pchejetski, D.; Saint-Laurent, N.; Brizuela, L.; Guilbeau-Frugier, C.; Delisle, M.-B.; Cuvillier, O.; Susini, C.; Bousquet, C. Targeting the Sphingolipid Metabolism to Defeat Pancreatic Cancer Cell Resistance to the Chemotherapeutic Gemcitabine Drug. Mol. Cancer 2009, 8, 809-820. [CrossRef] [PubMed]

531. Baran, Y.; Salas, A.; Senkal, C.E.; Gunduz, U.; Bielawski, J.; Obeid, L.M.; Ogretmen, B. Alterations of Ceramide/Sphingosine 1-Phosphate Rheostat Involved in the Regulation of Resistance to Imatinib-Induced Apoptosis in K562 Human Chronic Myeloid Leukemia Cells. J. Biol. Chem. 2007, 282, 10922-10934. [CrossRef] [PubMed]

532. Sukocheva, O.; Wang, L.; Verrier, E.; Vadas, M.A.; Xia, P. Restoring Endocrine Response in Breast Cancer Cells by Inhibition of the Sphingosine Kinase-1 Signaling Pathway. Endocrinology 2009, 150, 4484-4492. [CrossRef] [PubMed]

533. Madhunapantula, S.V.; Hengst, J.; Gowda, R.; Fox, T.E.; Yun, J.K.; Robertson, G.P. Targeting Sphingosine Kinase-1 to Inhibit Melanoma. Pigm. Cell Melanoma R 2012, 25, 259-274. [CrossRef]

534. Imbert, C.; Montfort, A.; Fraisse, M.; Marcheteau, E.; Gilhodes, J.; Martin, E.; Bertrand, F.; Marcellin, M.; Burlet-Schiltz, O.; de Peredo, A.G.; et al. Resistance of Melanoma to Immune Checkpoint Inhibitors Is Overcome by Targeting the Sphingosine Kinase-1. Nat. Commun. 2020, 11, 437. [CrossRef]

535. Ren, X.; Su, C. Sphingosine Kinase 1 Contributes to Doxorubicin Resistance and Glycolysis in Osteosarcoma. Mol. Med. Rep. 2020, 22, 2183-2190. [CrossRef]

536. Maczis, M.A.; Maceyka, M.; Waters, M.R.; Newton, J.; Singh, M.; Rigsby, M.F.; Turner, T.H.; Alzubi, M.A.; Harrell, J.C.; Milstien, S.; et al. Sphingosine Kinase 1 Activation by Estrogen Receptor A36 Contributes to Tamoxifen Resistance in Breast Cancer. J. Lipid Res. 2018, 59, 2297-2307. [CrossRef]

537. Xu, Y.; Dong, B.; Wang, J.; Zhang, J.; Xue, W.; Huang, Y. Sphingosine Kinase 1 Overexpression Contributes to Sunitinib Resistance in Clear Cell Renal Cell Carcinoma. Oncoimmunology 2018, 7, 1-10. [CrossRef] [PubMed] 
538. Wu, X.; Wu, Q.; Zhou, X.; Huang, J. SphK1 Functions Downstream of IGF-1 to Modulate IGF-1-Induced EMT, Migration and Paclitaxel Resistance of A549 Cells: A Preliminary in Vitro Study. J. Cancer 2019, 10, 4264-4269. [CrossRef] [PubMed]

539. Wang, S.; Liang, Y.; Chang, W.; Hu, B.; Zhang, Y. Triple Negative Breast Cancer Depends on Sphingosine Kinase 1 (SphK1)/Sphingosine-1-Phosphate (S1P)/Sphingosine 1-Phosphate Receptor 3 (S1PR3)/Notch Signaling for Metastasis. Med. Sci. Monit. Int. Med. J. Exp. Clin. Res. 2018, 24, 1912-1923. [CrossRef]

540. Wang, X.; Sun, Y.; Peng, X.; Naqvi, S.M.A.S.; Yang, Y.; Zhang, J.; Chen, M.; Chen, Y.; Chen, H.; Yan, H.; et al. The Tumorigenic Effect of Sphingosine Kinase 1 and Its Potential Therapeutic Target. Cancer Control. 2020, 27, 1073274820976664. [CrossRef] [PubMed]

541. Ruckhäberle, E.; Rody, A.; Engels, K.; Gaetje, R.; von Minckwitz, G.; Schiffmann, S.; Grösch, S.; Geisslinger, G.; Holtrich, U.; Karn, T.; et al. Microarray Analysis of Altered Sphingolipid Metabolism Reveals Prognostic Significance of Sphingosine Kinase 1 in Breast Cancer. Breast Cancer Res. Treat. 2008, 112, 41-52. [CrossRef]

542. Lim, K.G.; Tonelli, F.; Li, Z.; Lu, X.; Bittman, R.; Pyne, S.; Pyne, N.J. FTY720 Analogues as Sphingosine Kinase 1 Inhibitors Enzyme Inhibition Kinetics, Allosterism, Proteasomal Degradation, And Actin Rearrangement In Mcf-7 Breast Cancer Cells. J. Biol. Chem. 2011, 286, 18633-18640. [CrossRef]

543. Sharma, S.; Mathur, A.; Pradhan, S.; Singh, D.; Gupta, S. Fingolimod (FTY720): First Approved Oral Therapy for Multiple Sclerosis. J. Pharm. Pharm. 2011, 2, 49-51. [CrossRef]

544. Xu, H.; Wu, K.; Tian, Y.; Liu, Q.; Han, N.; Yuan, X.; Zhang, L.; Wu, G.S.; Wu, K. CD44 Correlates with Clinicopathological Characteristics and Is Upregulated by EGFR in Breast Cancer. Int. J. Oncol. 2016, 49, 1343-1350. [CrossRef]

545. Kawahara, S.; Otsuji, Y.; Nakamura, M.; Murakami, M.; Murate, T.; Matsunaga, T.; Kanoh, H.; Seishima, M.; Banno, Y.; Hara, A. Sphingosine Kinase 1 Plays a Role in the Upregulation of CD44 Expression through Extracellular Signal-Regulated Kinase Signaling in Human Colon Cancer Cells. Anti Cancer Drug 2013, 24, 473-483. [CrossRef]

546. Collina, F.; Bonito, M.D.; Bergolis, V.L.; Laurentiis, M.D.; Vitagliano, C.; Cerrone, M.; Nuzzo, F.; Cantile, M.; Botti, G. Prognostic Value of Cancer Stem Cells Markers in Triple-Negative Breast Cancer. Biomed. Res. Int. 2015, 2015, 1-10. [CrossRef] [PubMed]

547. Lin, M.Z.; Marzec, K.A.; Martin, J.L.; Baxter, R.C. The Role of Insulin-like Growth Factor Binding Protein-3 in the Breast Cancer Cell Response to DNA-Damaging Agents. Oncogene 2014, 33, 85-96. [CrossRef]

548. Schnute, M.E.; McReynolds, M.D.; Kasten, T.; Yates, M.; Jerome, G.; Rains, J.W.; Hall, T.; Chrencik, J.; Kraus, M.; Cronin, C.N.; et al. Modulation of Cellular S1P Levels with a Novel, Potent and Specific Inhibitor of Sphingosine Kinase-1. Biochem. J. 2012, 444, 79-88. [CrossRef] [PubMed]

549. Peyroche, A.; Antonny, B.; Robineau, S.; Acker, J.; Cherfils, J.; Jackson, C.L. Brefeldin A Acts to Stabilize an Abortive ARF-GDPSec7 Domain Protein Complex Involvement of Specific Residues of the Sec7 Domain. Mol. Cell 1999, 3, 275-285. [CrossRef]

550. Lu, H.; Zhou, Q.; He, J.; Jiang, Z.; Peng, C.; Tong, R.; Shi, J. Recent Advances in the Development of Protein-Protein Interactions Modulators: Mechanisms and Clinical Trials. Signal. Transduct. Target. 2020, 5, 213. [CrossRef]

551. Takayama, S.; Xie, Z.; Reed, J.C. An Evolutionarily Conserved Family of Hsp70/Hsc70 Molecular Chaperone Regulators. J. Biol. Chem. 1999, 274, 781-786. [CrossRef]

552. Rauch, J.N.; Zuiderweg, E.R.P.; Gestwicki, J.E. Non-Canonical Interactions between Heat Shock Cognate Protein 70 (Hsc70) and Bcl2-Associated Anthanogene (BAG) Co-Chaperones Are Important for Client Release. J. Biol. Chem. 2016, $291,19848-19857$. [CrossRef]

553. Wang, J.; Lou, P.; Lesniewski, R.; Henkin, J. Paclitaxel at Ultra Low Concentrations Inhibits Angiogenesis without Affecting Cellular Microtubule Assembly. Anti Cancer Drug 2003, 14, 13-19. [CrossRef]

554. Bollag, D.M.; McQueney, P.A.; Zhu, J.; Hensens, O.; Koupal, L.; Liesch, J.; Goetz, M.; Lazarides, E.; Woods, C.M. Epothilones, a New Class of Microtubule-Stabilizing Agents with a Taxol-like Mechanism of Action. Cancer Res. 1995, 55, $2325-2333$.

555. Lee, F.Y.; Borzilleri, R.; Fairchild, C.R.; Kim, S.H.; Long, B.H.; Reventos-Suarez, C.; Vite, G.D.; Rose, W.C.; Kramer, R.A. BMS247550: A Novel Epothilone Analog with a Mode of Action Similar to Paclitaxel but Possessing Superior Antitumor Efficacy. Clin. Cancer Res. Off. J. Am. Assoc. Cancer Res. 2001, 7, 1429-1437.

556. De Almeida, L.C.; Calil, F.A.; Machado-Neto, J.A.; Costa-Lotufo, L.V. DNA Damaging Agents and DNA Repair: From Carcinogenesis to Cancer Therapy. Cancer Genet. 2020, 252-253, 6-24. [CrossRef]

557. Yimit, A.; Adebali, O.; Sancar, A.; Jiang, Y. Differential Damage and Repair of DNA-Adducts Induced by Anti-Cancer Drug Cisplatin across Mouse Organs. Nat. Commun 2019, 10, 309. [CrossRef]

558. Minotti, G.; Menna, P.; Salvatorelli, E.; Cairo, G.; Gianni, L. Anthracyclines: Molecular Advances and Pharmacologic Developments in Antitumor Activity and Cardiotoxicity. Pharm. Rev. 2004, 56, 185-229. [CrossRef] [PubMed]

559. Xiang, T.; Ohashi, A.; Huang, Y.; Pandita, T.K.; Ludwig, T.; Powell, S.N.; Yang, Q. Negative Regulation of AKT Activation by BRCA1. Cancer Res. 2008, 68, 10040-10044. [CrossRef] [PubMed]

560. Galmarini, C.M.; Mackey, J.R.; Dumontet, C. Nucleoside Analogues and Nucleobases in Cancer Treatment. Lancet Oncol. 2002, 3 , 415-424. [CrossRef]

561. Von der Maase, H.; Hansen, S.W.; Roberts, J.T.; Dogliotti, L.; Oliver, T.; Moore, M.J.; Bodrogi, I.; Albers, P.; Knuth, A.; Lippert, C.M.; et al. Gemcitabine and Cisplatin Versus Methotrexate, Vinblastine, Doxorubicin, and Cisplatin in Advanced or Metastatic Bladder Cancer: Results of a Large, Randomized, Multinational, Multicenter, Phase III Study. J. Clin. Oncol. 2000, 18, 3068-3077. [CrossRef] [PubMed] 
562. Albain, K.S.; Nag, S.M.; Calderillo-Ruiz, G.; Jordaan, J.P.; Llombart, A.C.; Pluzanska, A.; Rolski, J.; Melemed, A.S.; Reyes-Vidal, J.M.; Sekhon, J.S.; et al. Gemcitabine Plus Paclitaxel Versus Paclitaxel Monotherapy in Patients with Metastatic Breast Cancer and Prior Anthracycline Treatment. J. Clin. Oncol. 2008, 26, 3950-3957. [CrossRef] [PubMed]

563. Sandler, A.B.; Nemunaitis, J.; Denham, C.; von Pawel, J.; Cormier, Y.; Gatzemeier, U.; Mattson, K.; Manegold, C.; Palmer, M.C.; Gregor, A.; et al. Phase III Trial of Gemcitabine Plus Cisplatin Versus Cisplatin Alone in Patients with Locally Advanced or Metastatic Non-Small-Cell Lung Cancer. J. Clin. Oncol. 2000, 18, 122. [CrossRef] [PubMed]

564. Mutch, D.G.; Orlando, M.; Goss, T.; Teneriello, M.G.; Gordon, A.N.; McMeekin, S.D.; Wang, Y.; Scribner, D.R.S., Jr.; Marciniack, M.; Naumann, R.W.; et al. Randomized Phase III Trial of Gemcitabine Compared with Pegylated Liposomal Doxorubicin in Patients with Platinum-Resistant Ovarian Cancer. J. Clin. Oncol. 2007, 25, 2811-2818. [CrossRef] [PubMed]

565. Ferrandina, G.; Ludovisi, M.; Lorusso, D.; Pignata, S.; Breda, E.; Savarese, A.; Medico, P.D.; Scaltriti, L.; Katsaros, D.; Priolo, D.; et al. Phase III Trial of Gemcitabine Compared with Pegylated Liposomal Doxorubicin in Progressive or Recurrent Ovarian Cancer. J. Clin. Oncol. 2008, 26, 890-896. [CrossRef] [PubMed]

566. Burris, H.A.; Moore, M.J.; Andersen, J.; Green, M.R.; Rothenberg, M.L.; Modiano, M.R.; Cripps, M.C.; Portenoy, R.K.; Storniolo, A.M.; Tarassoff, P.; et al. Improvements in Survival and Clinical Benefit with Gemcitabine as First-Line Therapy for Patients with Advanced Pancreas Cancer: A Randomized Trial. J. Clin. Oncol. 1997, 15, 2403-2413. [CrossRef]

567. Mini, E.; Nobili, S.; Caciagli, B.; Landini, I.; Mazzei, T. Cellular Pharmacology of Gemcitabine. Ann. Oncol. 2006, 17, v7-v12. [CrossRef] [PubMed]

568. Gandhi, V.; Plunkett, W. Modulatory Activity of 2',2'-Difluorodeoxycytidine on the Phosphorylation and Cytotoxicity of Arabinosyl Nucleosides. Cancer Res. 1990, 50, 3675-3680. [PubMed]

569. Heinemann, V.; Xu, Y.Z.; Chubb, S.; Sen, A.; Hertel, L.W.; Grindey, G.B.; Plunkett, W. Inhibition of Ribonucleotide Reduction in CCRF-CEM Cells by 2',2'-Difluorodeoxycytidine. Mol. Pharm. 1990, 38, 567-572.

570. Baker, C.H.; Banzon, J.; Bollinger, J.M.; Stubbe, J.; Samano, V.; Robins, M.J.; Lippert, B.; Jarvi, E.; Resvick, R. $2^{\prime}$-Deoxy-2' Methylenecytidine and 2'-Deoxy-2', 2'-Difluorocytidine 5'-Diphosphates: Potent Mechanism-Based Inhibitors of Ribonucleotide Reductase. J. Med. Chem. 1991, 34, 1879-1884. [CrossRef]

571. Honeywell, R.J.; van Haperen, V.W.T.R.; Veerman, G.; Smid, K.; Peters, G.J. Inhibition of Thymidylate Synthase by $2^{\prime}, 2^{\prime}$-Difluoro2'-Deoxycytidine (Gemcitabine) and Its Metabolite 2',2'-Difluoro-2'-Deoxyuridine. Int. J. Biochem. Cell Biol. 2015, 60, 73-81. [CrossRef]

572. Holliger, P.; Prospero, T.; Winter, G. "Diabodies": Small Bivalent and Bispecific Antibody Fragments. Proc. Natl. Acad. Sci. USA 1993, 90, 6444-6448. [CrossRef]

573. Han, W.; Pan, H.; Chen, Y.; Sun, J.; Wang, Y.; Li, J.; Ge, W.; Feng, L.; Lin, X.; Wang, X.; et al. EGFR Tyrosine Kinase Inhibitors Activate Autophagy as a Cytoprotective Response in Human Lung Cancer Cells. PLoS ONE 2011, 6, e18691. [CrossRef] [PubMed]

574. Zhao, Z.-Q.; Yu, Z.-Y.; Li, J.; Ouyang, X.-N. Gefitinib Induces Lung Cancer Cell Autophagy and Apoptosis via Blockade of the PI3K/AKT/MTOR Pathway. Oncol. Lett 2016, 12, 63-68. [CrossRef]

575. Mah, L.-J.; El-Osta, A.; Karagiannis, T.C. ГH2AX: A Sensitive Molecular Marker of DNA Damage and Repair. Leukemia 2010, 24, 679-686. [CrossRef] [PubMed]

576. Said, A.; Bock, S.; Lajqi, T.; Müller, G.; Weindl, G. Chloroquine Promotes IL-17 Production by CD4+ T Cells via P38-Dependent IL-23 Release by Monocyte-Derived Langerhans-like Cells. J. Immunol. 2014, 193, 6135-6143. [CrossRef]

577. Hansen, T.E.; Johansen, T. Following Autophagy Step by Step. BMC Biol. 2011, 9, 39. [CrossRef]

578. Bui, N.-L.-C.; Pandey, V.; Zhu, T.; Ma, L.; Basappa; Lobie, P.E. Bad Phosphorylation as a Target of Inhibition in Oncology. Cancer Lett. 2018, 415, 177-186. [CrossRef]

579. Yang, X.-J.; Xi, Y.-M.; Li, Z.-J. Icaritin: A Novel Natural Candidate for Hematological Malignancies Therapy. Biomed. Res. Int. 2019, 2019, 1-7. [CrossRef] [PubMed]

580. Wang, X.; Zheng, N.; Dong, J.; Wang, X.; Liu, L.; Huang, J. Estrogen Receptor-A36 Is Involved in Icaritin Induced Growth Inhibition of Triple-Negative Breast Cancer Cells. J. Steroid Biochem. Mol. Biol. 2017, 171, 318-327. [CrossRef]

581. Daley, J. Gene Therapy Arrives. Nature 2019, 576, S12-S13. [CrossRef]

582. Bergsbaken, T.; Fink, S.L.; Cookson, B.T. Pyroptosis: Host Cell Death and Inflammation. Nat. Rev. Microbiol. 2009, 7, 99-109. [CrossRef]

583. Ding, J.; Wang, K.; Liu, W.; She, Y.; Sun, Q.; Shi, J.; Sun, H.; Wang, D.-C.; Shao, F. Pore-Forming Activity and Structural Autoinhibition of the Gasdermin Family. Nature 2016, 535, 111-116. [CrossRef]

584. Wang, Y.; Gao, W.; Shi, X.; Ding, J.; Liu, W.; He, H.; Wang, K.; Shao, F. Chemotherapy Drugs Induce Pyroptosis through Caspase-3 Cleavage of a Gasdermin. Nature 2017, 547, 99-103. [CrossRef]

585. Jia, H.; Truica, C.I.; Wang, B.; Wang, Y.; Ren, X.; Harvey, H.A.; Song, J.; Yang, J.-M. Immunotherapy for Triple-Negative Breast Cancer: Existing Challenges and Exciting Prospects. Drug Resist. Update 2017, 32, 1-15. [CrossRef]

586. Li, Z.; Qiu, Y.; Lu, W.; Jiang, Y.; Wang, J. Immunotherapeutic Interventions of Triple Negative Breast Cancer. J. Transl. Med. 2018, 16, 147. [CrossRef]

587. Katz, H.; Alsharedi, M. Immunotherapy in Triple-Negative Breast Cancer. Med. Oncol. 2018, 35, 13. [CrossRef]

588. Marra, A.; Viale, G.; Curigliano, G. Recent Advances in Triple Negative Breast Cancer: The Immunotherapy Era. BMC Med. 2019, 17, 90. [CrossRef] 
589. Wahby, S.; Fashoyin-Aje, L.; Osgood, C.L.; Cheng, J.; Fiero, M.H.; Zhang, L.; Tang, S.; Hamed, S.S.; Song, P.; Charlab, R.; et al. FDA Approval Summary: Accelerated Approval of Sacituzumab Govitecan-Hziy for Third-Line Treatment of Metastatic TripleNegative Breast Cancer. Clin. Cancer Res. 2021. [CrossRef]

590. Bardia, A.; Mayer, I.A.; Vahdat, L.T.; Tolaney, S.M.; Isakoff, S.J.; Diamond, J.R.; O'Shaughnessy, J.; Moroose, R.L.; Santin, A.D.; Abramson, V.G.; et al. Sacituzumab Govitecan-Hziy in Refractory Metastatic Triple-Negative Breast Cancer. N. Engl. J. Med. 2019, 380, 741-751. [CrossRef] [PubMed]

591. FDA. FDA Grants Regular Approval to Sacituzumab Govitecan for Triple-Negative Breast Cancer. Available online: https: / / www.fda.gov/drugs/resources-information-approved-drugs/fda-grants-regular-approval-sacituzumab-govitecantriple-negative-breast-cancer\#: \{\}:text=On \%20April \%207\%2C \%202021\%2C \%20the,least $\% 20$ one $\% 20$ of $\% 20$ them $\% 20$ for (accessed on 13 April 2021).

592. Larson, R.C.; Maus, M.V. Recent Advances and Discoveries in the Mechanisms and Functions of CAR T Cells. Nat. Rev. Cancer 2021, 21, 145-161. [CrossRef] [PubMed]

593. Zhang, C.; Oberoi, P.; Oelsner, S.; Waldmann, A.; Lindner, A.; Tonn, T.; Wels, W.S. Chimeric Antigen Receptor-Engineered NK-92 Cells: An Off-the-Shelf Cellular Therapeutic for Targeted Elimination of Cancer Cells and Induction of Protective Antitumor Immunity. Front. Immunol. 2017, 8, 533. [CrossRef] [PubMed]

594. Klichinsky, M.; Ruella, M.; Shestova, O.; Lu, X.M.; Best, A.; Zeeman, M.; Schmierer, M.; Gabrusiewicz, K.; Anderson, N.R.; Petty, N.E.; et al. Human Chimeric Antigen Receptor Macrophages for Cancer Immunotherapy. Nat. Biotechnol. 2020, 38, 947-953. [CrossRef]

595. June, C.H.; O'Connor, R.S.; Kawalekar, O.U.; Ghassemi, S.; Milone, M.C. CAR T Cell Immunotherapy for Human Cancer. Science 2018, 359, 1361-1365. [CrossRef]

596. Bagley, S.J.; O’Rourke, D.M. Clinical Investigation of CAR T Cells for Solid Tumors: Lessons Learned and Future Directions. Pharm. Ther. 2020, 205, 107419. [CrossRef]

597. Dees, S.; Ganesan, R.; Singh, S.; Grewal, I.S. Emerging CAR-T Cell Therapy for the Treatment of Triple-Negative Breast Cancer. Mol. Cancer 2020, 19, 2409-2421. [CrossRef] [PubMed]

598. Bruhns, P.; Iannascoli, B.; England, P.; Mancardi, D.A.; Fernandez, N.; Jorieux, S.; Daëron, M. Specificity and Affinity of Human Fc $\gamma$ Receptors and Their Polymorphic Variants for Human IgG Subclasses. Blood 2009, 113, 3716-3725. [CrossRef]

599. Schneider-Merck, T.; van Bueren, J.J.L.; Berger, S.; Rossen, K.; van Berkel, P.H.C.; Derer, S.; Beyer, T.; Lohse, S.; Bleeker, W.K.; Peipp, M.; et al. Human IgG2 Antibodies against Epidermal Growth Factor Receptor Effectively Trigger Antibody-Dependent Cellular Cytotoxicity but, in Contrast to IgG1, Only by Cells of Myeloid Lineage. J. Immunol. 2010, 184, 512-520. [CrossRef]

600. Ramello, M.C.; Haura, E.B.; Abate-Daga, D. CAR-T Cells and Combination Therapies: What's next in the Immunotherapy Revolution? Pharm. Res. 2018, 129, 194-203. [CrossRef] [PubMed]

601. Ludwig, L.M.; Nassin, M.L.; Hadji, A.; LaBelle, J.L. Killing Two Cells with One Stone: Pharmacologic BCL-2 Family Targeting for Cancer Cell Death and Immune Modulation. Front. Pediatrics 2016, 4, 135. [CrossRef] [PubMed]

602. Dinh, T.N.; Onea, A.S.; Jazirehi, A.R. Combination of Celecoxib (Celebrex ${ }^{\circledR}$ ) and CD19 CAR-Redirected CTL Immunotherapy for the Treatment of B-Cell Non-Hodgkin's Lymphomas. Am. J. Clin. Exp. Immunol. 2017, 6, 27-42.

603. Joshi, S.; Durden, D.L. Combinatorial Approach to Improve Cancer Immunotherapy: Rational Drug Design Strategy to Simultaneously Hit Multiple Targets to Kill Tumor Cells and to Activate the Immune System. J. Oncol. 2019, 2019, 1-18. [CrossRef]

604. He, X.; Xu, C. Immune Checkpoint Signaling and Cancer Immunotherapy. Cell Res. 2020, 30, 660-669. [CrossRef]

605. Ishida, Y.; Agata, Y.; Shibahara, K.; Honjo, T. Induced Expression of PD-1, a Novel Member of the Immunoglobulin Gene Superfamily, upon Programmed Cell Death. EMBO J. 1992, 11, 3887-3895. [CrossRef]

606. Mittendorf, E.A.; Philips, A.V.; Meric-Bernstam, F.; Qiao, N.; Wu, Y.; Harrington, S.; Su, X.; Wang, Y.; Gonzalez-Angulo, A.M.; Akcakanat, A.; et al. PD-L1 Expression in Triple-Negative Breast Cancer. Cancer Immunol. 2014, 2, 361-370. [CrossRef]

607. Narayan, P.; Wahby, S.; Gao, J.J.; Amiri-Kordestani, L.; Ibrahim, A.; Bloomquist, E.; Tang, S.; Xu, Y.; Liu, J.; Fu, W.; et al. FDA Approval Summary: Atezolizumab Plus Paclitaxel Protein-Bound for the Treatment of Patients with Advanced or Metastatic TNBC Whose Tumors Express PD-L1. Clin. Cancer Res. 2020, 26, 2284-2289. [CrossRef] [PubMed]

608. FDA. FDA Grants Accelerated Approval to Pembrolizumab for Locally Recurrent Unresectable or Metastatic Triple Negative Breast Cancer. Available online: https:/ / www.fda.gov/drugs/drug-approvals-and-databases/fda-grants-accelerated-approvalpembrolizumab-locally-recurrent-unresectable-or-metastatic-triple (accessed on 13 April 2021).

609. Boonstra, M.C.; Geus, S.W.L.D.; Prevoo, H.A.J.M.; Hawinkels, L.J.A.C.; Velde, C.J.H.V.D.; Kuppen, P.J.K.; Vahrmeijer, A.L.; Sier, C.F.M. Selecting Targets for Tumor Imaging: An Overview of Cancer-Associated Membrane Proteins. Biomark. Cancer 2016, 8 , BIC.S38542. [CrossRef]

610. Yu, B.; Tai, H.C.; Xue, W.; Lee, L.J.; Lee, R.J. Receptor-Targeted Nanocarriers for Therapeutic Delivery to Cancer. Mol. Membr. Biol. 2010, 27, 286-298. [CrossRef] [PubMed]

611. Park, K.-S.; Bandeira, E.; Shelke, G.V.; Lässer, C.; Lötvall, J. Enhancement of Therapeutic Potential of Mesenchymal Stem Cell-Derived Extracellular Vesicles. Stem Cell Res. 2019, 10, 288. [CrossRef]

612. Yeo, R.W.Y.; Lai, R.C.; Zhang, B.; Tan, S.S.; Yin, Y.; Teh, B.J.; Lim, S.K. Mesenchymal Stem Cell: An Efficient Mass Producer of Exosomes for Drug Delivery. Adv. Drug Deliv. Rev. 2013, 65, 336-341. [CrossRef]

613. Jain, A.; Singh, S.K.; Arya, S.K.; Kundu, S.C.; Kapoor, S. Protein Nanoparticles: Promising Platforms for Drug Delivery Applications. Acs Biomater. Sci. Eng 2018, 4, 3939-3961. [CrossRef] [PubMed] 
614. Hong, S.; Choi, D.W.; Kim, H.N.; Park, C.G.; Lee, W.; Park, H.H. Protein-Based Nanoparticles as Drug Delivery Systems. Pharmaceutics 2020, 12, 604. [CrossRef] [PubMed]

615. Kianfar, E. Protein Nanoparticles in Drug Delivery: Animal Protein, Plant Proteins and Protein Cages, Albumin Nanoparticles. J. Nanobiotechnol. 2021, 19, 159. [CrossRef]

616. Imai, Y.; Leung, C.K.H.; Friesen, H.G.; Shiu, R.P.C. Epidermal Growth Factor Receptors and Effect of Epidermal Growth Factor on Growth of Human Breast Cancer Cells in Long-Term Tissue Culture. Cancer Res. 1982, 42, 4394-4398. [PubMed]

617. Nestor, M.; Ekberg, T.; Dring, J.; van Dongen, G.A.M.S.; Wester, K.; Tolmachev, V.; Anniko, M. Quantification of CD44v6 and EGFR Expression in Head and Neck Squamous Cell Carcinomas Using a Single-Dose Radioimmunoassay. Tumor Biol. 2007, 28, 253-263. [CrossRef] [PubMed]

618. Drago, J.Z.; Modi, S.; Chandarlapaty, S. Unlocking the Potential of Antibody-Drug Conjugates for Cancer Therapy. Nat. Rev. Clin. Oncol. 2021, 18, 327-344. [CrossRef] [PubMed]

619. Khongorzul, P.; Ling, C.J.; Khan, F.U.; Ihsan, A.U.; Zhang, J. Antibody-Drug Conjugates: A Comprehensive Review. Mol. Cancer Res. 2020, 18, 3-19. [CrossRef]

620. Biteghe, F.A.N.; Mungra, N.; Chalomie, N.E.T.; Ndong, J.D.L.C.; Engohang-Ndong, J.; Vignaux, G.; Padayachee, E.; Naran, K.; Barth, S. Advances in Epidermal Growth Factor Receptor Specific Immunotherapy: Lessons to Be Learned from Armed Antibodies. Oncotarget 2020, 11, 3531-3557. [CrossRef]

621. Large, D.E.; Soucy, J.R.; Hebert, J.; Auguste, D.T. Advances in Receptor-Mediated, Tumor-Targeted Drug Delivery. Adv. Ther. 2019, 2, 1800091. [CrossRef]

622. Kunjiappan, S.; Pavadai, P.; Vellaichamy, S.; Pandian, S.R.K.; Ravishankar, V.; Palanisamy, P.; Govindaraj, S.; Srinivasan, G.; Premanand, A.; Sankaranarayanan, M.; et al. Surface Receptor-mediated Targeted Drug Delivery Systems for Enhanced Cancer Treatment: A State-of-the-art Review. Drug Dev. Res. 2021, 82, 309-340. [CrossRef]

623. Shu, D.; Li, H.; Shu, Y.; Xiong, G.; Carson, W.E.; Haque, F.; Xu, R.; Guo, P. Systemic Delivery of Anti-MiRNA for Suppression of Triple Negative Breast Cancer Utilizing RNA Nanotechnology. ACS Nano 2015, 9, 9731-9740. [CrossRef]

624. Camorani, S.; Crescenzi, E.; Fedele, M.; Cerchia, L. Oligonucleotide Aptamers against Tyrosine Kinase Receptors: Prospect for Anticancer Applications. Biochim. Biophys. Acta Bba Rev. Cancer 2018, 1869, 263-277. [CrossRef]

625. Guo, S.; Vieweger, M.; Zhang, K.; Yin, H.; Wang, H.; Li, X.; Li, S.; Hu, S.; Sparreboom, A.; Evers, B.M.; et al. Ultra-Thermostable RNA Nanoparticles for Solubilizing and High-Yield Loading of Paclitaxel for Breast Cancer Therapy. Nat. Commun. 2020, 11, 972. [CrossRef] [PubMed]

626. Yang, L.; Mao, H.; Wang, Y.A.; Cao, Z.; Peng, X.; Wang, X.; Duan, H.; Ni, C.; Yuan, Q.; Adams, G.; et al. Single Chain Epidermal Growth Factor Receptor Antibody Conjugated Nanoparticles for in Vivo Tumor Targeting and Imaging. Small 2009, 5, 235-243. [CrossRef] [PubMed]

627. Master, A.M.; Gupta, A.S. EGF Receptor-Targeted Nanocarriers for Enhanced Cancer Treatment. Nanomedicine 2012, 7, 1895-1906. [CrossRef] [PubMed]

628. Sun, S.; Ding, Z.; Yang, X.; Zhao, X.; Zhao, M.; Gao, L.; Chen, Q.; Xie, S.; Liu, A.; Yin, S.; et al. Nanobody: A Small Antibody with Big Implications for Tumor Therapeutic Strategy. Int. J. Nanomed. 2021, 16, 2337-2356. [CrossRef]

629. Tiede, C.; Bedford, R.; Heseltine, S.J.; Smith, G.; Wijetunga, I.; Ross, R.; AlQallaf, D.; Roberts, A.P.; Balls, A.; Curd, A.; et al. Affimer Proteins Are Versatile and Renewable Affinity Reagents. Elife 2017, 6, e24903. [CrossRef]

630. Feiner, R.C.; Kemker, I.; Krutzke, L.; Allmendinger, E.; Mandell, D.J.; Sewald, N.; Kochanek, S.; Müller, K.M. EGFR-Binding Peptides: From Computational Design towards Tumor-Targeting of Adeno-Associated Virus Capsids. Int. J. Mol. Sci. 2020, 21, 9535. [CrossRef] [PubMed]

631. Smith, S.A.; Selby, L.I.; Johnston, A.P.R.; Such, G.K. The Endosomal Escape of Nanoparticles: Toward More Efficient Cellular Delivery. Bioconjugate Chem. 2018, 30, 263-272. [CrossRef] [PubMed]

632. Cupic, K.I.; Rennick, J.J.; Johnston, A.P.; Such, G.K. Controlling Endosomal Escape Using Nanoparticle Composition: Current Progress and Future Perspectives. Nanomedicine 2019, 14, 215-223. [CrossRef] [PubMed]

633. Ahmad, A.; Khan, J.M.; Haque, S. Strategies in the Design of Endosomolytic Agents for Facilitating Endosomal Escape in Nanoparticles. Biochimie 2019, 160, 61-75. [CrossRef] [PubMed]

634. Gholam, D.; Chebib, A.; Hauteville, D.; Bralet, M.-P.; Jasmin, C. Combined Paclitaxel and Cetuximab Achieved a Major Response on the Skin Metastases of a Patient with Epidermal Growth Factor Receptor-Positive, Estrogen Receptor-Negative, Progesterone Receptor-Negative and Human Epidermal Growth Factor Receptor-2-Negative (Triple-Negative) Breast Cancer. Anti Cancer Drug 2007, 18, 835-837. [CrossRef]

635. Carey, L.A.; Rugo, H.S.; Marcom, P.K.; Mayer, E.L.; Esteva, F.J.; Ma, C.X.; Liu, M.C.; Storniolo, A.M.; Rimawi, M.F.; Forero-Torres, A.; et al. TBCRC 001: Randomized Phase II Study of Cetuximab in Combination with Carboplatin in Stage IV Triple-Negative Breast Cancer. J. Clin. Oncol. 2012, 30, 2615-2623. [CrossRef]

636. Baselga, J.; Gómez, P.; Greil, R.; Braga, S.; Climent, M.A.; Wardley, A.M.; Kaufman, B.; Stemmer, S.M.; Pêgo, A.; Chan, A.; et al. Randomized Phase II Study of the Anti-Epidermal Growth Factor Receptor Monoclonal Antibody Cetuximab with Cisplatin Versus Cisplatin Alone in Patients with Metastatic Triple-Negative Breast Cancer. J. Clin. Oncol. 2013, 31, 2586-2592. [CrossRef]

637. Nabholtz, J.M.; Chalabi, N.; Radosevic-Robin, N.; Dauplat, M.M.; Mouret-Reynier, M.A.; Praagh, I.V.; Servent, V.; Jacquin, J.; Benmammar, K.E.; Kullab, S.; et al. Multicentric Neoadjuvant Pilot Phase II Study of Cetuximab Combined with Docetaxel in Operable Triple Negative Breast Cancer. Int. J. Cancer 2016, 138, 2274-2280. [CrossRef] [PubMed] 
638. Trédan, O.; Campone, M.; Jassem, J.; Vyzula, R.; Coudert, B.; Pacilio, C.; Prausova, J.; Hardy-Bessard, A.-C.; Arance, A.; Mukhopadhyay, P.; et al. Ixabepilone Alone or with Cetuximab as First-Line Treatment for Advanced/Metastatic Triple-Negative Breast Cancer. Clin. Breast Cancer 2015, 15, 8-15. [CrossRef]

639. Crozier, J.A.; Advani, P.P.; LaPlant, B.; Hobday, T.; Jaslowski, A.J.; Moreno-Aspitia, A.; Perez, E.A. N0436 (Alliance): A Phase II Trial of Irinotecan with Cetuximab in Patients with Metastatic Breast Cancer Previously Exposed to Anthracycline and/or Taxane-Containing Therapy. Clin. Breast Cancer 2016, 16, 23-30. [CrossRef]

640. Nabholtz, J.M.; Abrial, C.; Mouret-Reynier, M.A.; Dauplat, M.M.; Weber, B.; Gligorov, J.; Forest, A.M.; Tredan, O.; Vanlemmens, L.; Petit, T.; et al. Multicentric Neoadjuvant Phase II Study of Panitumumab Combined with an Anthracycline/Taxane-Based Chemotherapy in Operable Triple-Negative Breast Cancer: Identification of Biologically Defined Signatures Predicting Treatment Impact. Ann. Oncol. 2014, 25, 1570-1577. [CrossRef] [PubMed]

641. Cowherd, S.; Miller, L.D.; Melin, S.A.; Akman, S.; Isom, S.; Cole, J.; Pullikuth, A.; Lawrence, J.A. A Phase II Clinical Trial of Weekly Paclitaxel and Carboplatin in Combination with Panitumumab in Metastatic Triple Negative Breast Cancer. Cancer Biol. 2015, 16, 678-683. [CrossRef] [PubMed]

642. Lynch, T.J.; Patel, T.; Dreisbach, L.; McCleod, M.; Heim, W.J.; Hermann, R.C.; Paschold, E.; Iannotti, N.O.; Dakhil, S.; Gorton, S.; et al. Cetuximab and First-Line Taxane/Carboplatin Chemotherapy in Advanced Non-Small-Cell Lung Cancer: Results of the Randomized Multicenter Phase III Trial BMS099. J. Clin. Oncol. 2010, 28, 911-917. [CrossRef] [PubMed]

643. O'Shaughnessy, J.A. Highlights in Metastatic Breast Cancer from the 2013 San Antonio Breast Cancer Symposium (SABCS). Clin. Adv. Hematol. Oncol. 2014, 12, 3-17.

644. Pirker, R.; Pereira, J.R.; Szczesna, A.; von Pawel, J.; Krzakowski, M.; Ramlau, R.; Vynnychenko, I.; Park, K.; Yu, C.-T.; Ganul, V.; et al. Cetuximab plus Chemotherapy in Patients with Advanced Non-Small-Cell Lung Cancer (FLEX): An Open-Label Randomised Phase III Trial. Lancet 2009, 373, 1525-1531. [CrossRef]

645. Matsuda, N.; Wang, X.; Lim, B.; Krishnamurthy, S.; Alvarez, R.H.; Willey, J.S.; Parker, C.A.; Song, J.; Shen, Y.; Hu, J.; et al. Safety and Efficacy of Panitumumab Plus Neoadjuvant Chemotherapy in Patients with Primary HER2-Negative Inflammatory Breast Cancer. JAMA Oncol. 2018, 4, 1207. [CrossRef] [PubMed]

646. Yardley, D.A.; Ward, P.J.; Daniel, B.R.; Eakle, J.F.; Lamar, R.E.; Lane, C.M.; Hainsworth, J.D. Panitumumab, Gemcitabine, and Carboplatin as Treatment for Women with Metastatic Triple-Negative Breast Cancer: A Sarah Cannon Research Institute Phase II Trial. Clin. Breast Cancer 2016, 16, 349-355. [CrossRef] [PubMed]

647. Bernsdorf, M.; Ingvar, C.; Jörgensen, L.; Tuxen, M.K.; Jakobsen, E.H.; Saetersdal, A.; Kimper-Karl, M.L.; Kroman, N.; Balslev, E.; Ejlertsen, B. Effect of Adding Gefitinib to Neoadjuvant Chemotherapy in Estrogen Receptor Negative Early Breast Cancer in a Randomized Phase II Trial. Breast Cancer Res. Tr 2011, 126, 463-470. [CrossRef]

648. Stringer-Reasor, E.M.; May, J.E.; Olariu, E.; Caterinicchia, V.; Li, Y.; Chen, D.; Manna, D.L.D.; Rocque, G.B.; Vaklavas, C.; Falkson, C.I.; et al. An Open-Label, Pilot Study of Veliparib and Lapatinib in Patients with Metastatic, Triple-Negative Breast Cancer. Breast Cancer Res. 2021, 23, 30. [CrossRef] [PubMed]

649. Fenn, K.; Maurer, M.; Lee, S.M.; Crew, K.D.; Trivedi, M.S.; Accordino, M.K.; Hershman, D.L.; Kalinsky, K. Phase I Study of Erlotinib and Metformin in Metastatic Triple Negative Breast Cancer. Clin. Breast Cancer 2019, 20, 80-86. [CrossRef] 